\title{
Structure and Function of NzeB, a Versatile C-C and C-N Bond Forming Diketopiperazine Dimerase
}

Vikram V. Shende, Yogan Khatri, Sean A. Newmister, Jacob N. Sanders, Petra Lindovska, Fengan Yu, Tyler J. Doyon, Justin Kim, Mohammad Movassaghi, Kendall N Houk, David H. Sherman

Submitted date: $14 / 05 / 2020$ - Posted date: 15/05/2020

Licence: CC BY-NC-ND 4.0

Citation information: Shende, Vikram V.; Khatri, Yogan; Newmister, Sean A.; Sanders, Jacob N.; Lindovska, Petra; Yu, Fengan; et al. (2020): Structure and Function of NzeB, a Versatile C-C and C-N Bond Forming Diketopiperazine Dimerase. ChemRxiv. Preprint. https://doi.org/10.26434/chemrxiv.12307439.v1

This report details the discovery and characterization of a versatile bacterial cytochrome P450, NzeB, which catalyzes the dimerization of diketopiperazines via enzymatic $\mathrm{C}-\mathrm{H}$ functionalization. This includes the first high-resolution crystal structure of a diketopiperazine dimerase, which along with active site via mutagenesis and quantum mechanical calculations, provides insight into the selectivity and mechanism of these enzymes.

File list (2)

NzeB_2020_05_14.pdf (32.32 MiB)

view on ChemRxiv • download file

NzeB_SI_2020_05_14_Combined.pdf (8.25 MiB)

view on ChemRxiv - download file 


\title{
Structure and Function of NzeB, a Versatile C-C and $\mathrm{C}-\mathrm{N}$ Bond Forming Diketopiperazine Dimerase
}

\author{
Vikram V. Shende, ${ }^{\perp}$ Yogan Khatri, ${ }^{+}$Sean A. Newmister, ${ }^{\dagger}$ Jacob N. Sanders, ${ }^{\theta}$ Petra Lindovska, ${ }^{\Delta}$ Fengan Yu, ${ }^{\perp}$ Tyler \\ J. Doyon, ${ }^{\perp}$ Justin Kim, ${ }^{\Delta}$ K. N. Houk, ${ }^{\theta, *}$ Mohammad Movassaghi, ${ }^{\Delta, *}$ and David H. Sherman ${ }^{\perp, \Psi_{*, j}, *}$ \\ ${ }^{\dagger}$ Life Sciences Institute, ${ }^{\mathrm{Y}}$ Department of Medicinal Chemistry, ${ }^{\perp}$ Program in Chemical Biology, "Department of Chemistry, and $\mathrm{se}$ - \\ partment of Microbiology \& Immunology, University of Michigan, Ann Arbor, Michigan, 48109, United States \\ ${ }^{\Delta}$ Department of Chemistry, Massachusetts Institute of Technology, Cambridge, Massachusetts, 02139, United States \\ ${ }^{\theta}$ Department of Chemistry, University of California Los Angeles, Los Angeles, California, 90095, United States
}

KEYWORDS cytochrome P450, diketopiperazine, diketopiperazine dimers, natural product biosynthesis, $C-H$ functionalization

\begin{abstract}
The dimeric diketopiperazine (DKPs) alkaloids are a diverse family of natural products (NPs) whose unique structural architectures and biological activities have inspired the development of new synthetic methodology to access these molecules. However, catalystcontrolled methods that enable the selective formation of constitutional and stereoisomeric dimers from a single monomer are lacking. To resolve this long-standing synthetic challenge, we sought to characterize the biosynthetic enzymes that assemble these NPs for application in biocatalytic syntheses. Genome mining enabled identification of the cytochrome P450, NzeB (derived from Streptomyces sp. NRRL F5053), which catalyzes both intermolecular carbon-carbon $(\mathrm{C}-\mathrm{C})$ and carbon-nitrogen $(\mathrm{C}-\mathrm{N})$ bond formation, generating all currently known DKP dimer scaffolds isolated from bacterial sources. To identify the molecular basis for the flexible site-, stereo-, and chemoselectivity of NzeB, we obtained high-resolution crystal structures (1.5 $)$ of the protein in complex with native and non-native substrates. This, to our knowledge, represents the first crystal structure of an oxidase catalyzing direct, intermolecular $\mathrm{C}-\mathrm{H}$ amination. Site-directed mutagenesis was employed to assess the role individual active site residues play in guiding selective DKP dimerization. Finally, computational approaches were employed to evaluate plausible mechanisms regarding NzeB function and its ability to catalyze both $\mathrm{C}-\mathrm{C}$ and $\mathrm{C}-\mathrm{N}$ bond formation. These results provide a structural and computational rationale for the catalytic versatility of $\mathrm{NzeB}$, as well as new insights into variables that control selectivity of CYP450 diketopiperazine dimerases.
\end{abstract}

The tryptophan-linked dimeric diketopiperazine (DKP) alkaloids are a family of over 50 natural products isolated from bacteria, fungi, and plants. ${ }^{1-4}$ Their dimerization modes encompass an array of stereo- and regiochemical connectivities, and potent biological activity of these natural products elicited the development and application of effective methods for their total synthesis. ${ }^{3-5}$ In particular, biomimetic strategies enabled access to $C_{2}$-symmetrical dimers, such as (-)-ditryptophenaline (2, Figure 1$)$, wherein two identical monomers are coupled at a late-stage resulting in highly convergent and efficient syntheses. ${ }^{6,7}$ However, the chemical synthesis of structurally challenging non-symmetrical DKP dimers such as (+)-naseseazines A (1) and B (2), ${ }^{8,9}(-)$-naseseazine C $(4),{ }^{10,11}$ and the related alkaloid (-)-aspergilazine A (5), ${ }^{10,12}$ continues to inspire the development of new synthetic routes. ${ }^{4}, 9,13-16$ The synthesis of structurally complex non-symmetrical dimeric DKPs requires independent synthesis of appropriately substituted precursors to allow selective formation of a key $\mathrm{C}-\mathrm{C}$ or $\mathrm{C}-\mathrm{N}$ bond that adjoin the two subunits. ${ }^{9,17,18}$ Seeking to develop an efficient approach towards DKP dimer assembly, we envisioned a process wherein the site- and stereochemical outcome of dimerization would be dictated by the biocatalyst, and a single DKP monomer could dimerized selectively to a variety of site- and stereo-isomeric products.
While dozens of fungal dimeric diketopiperazine natural products have been reported since the isolation of 2, ${ }^{1-4,6}$ fungal biosynthetic gene clusters (BGCs) responsible for dimerization of DKPs have only recently been identified, and have been limited to biosynthesis of $C_{2}$ symmetrical dimers. ${ }^{19}$ Characterization of these BGCs identified cytochromes $\mathrm{P} 450$ that mediate the oxidative convergence of two DKP monomers to generate $C_{2}$ symmetrical dimers, validating long-standing hypotheses proposed in isolation, characterization, and total syntheses of these molecules. ${ }^{19}$ While the majority of DKP dimers have been isolated from fungi, marine actinomycetes have recently emerged as a new source for non-symmetrical dimeric DKP natural products. Notably, bacterial DKP dimers display coupling patterns distinct from their fungal counterparts, with the exception of 4, a rare example of a secondary metabolite isolated from both fungal and bacterial sources. ${ }^{8,10,11}$ Contemporaneous efforts to identify DKP dimer biosynthetic gene clusters from bacteria have led to the recent identification of gene clusters for dimers 3 (nasc $)^{20}$ (Streptomyces sp. CMB-MQ030), and the (+)-naseseazines A (1) and B (2) (nas) and (-)-aspergilazine A (5) gene clusters (Streptomyces sp. NRRL-S1868). ${ }^{21}$ While functional characterization of these BGCs have provided insight into the biogenesis of these natural products, the molecular basis for the selective formation of nonsymmetrical dimers by these $\mathrm{P} 450$ s has remained elusive. 
Herein, we report the identification of NzeB, a versatile P450 that catalyzes both $\mathrm{C}-\mathrm{C}$ and $\mathrm{C}-\mathrm{N}$ bond formation through bioinformatics and sequence similarity network (SSN) analysis. Structural and functional characterization of NzeB provides the first insights into the molecular basis of selectivity displayed by the DKP dimerizing class of P450s. Quantum mechanics (QM) calculations were performed to elucidate plausible mechanisms for NzeB catalyzed $\mathrm{C}-\mathrm{C}$ and $\mathrm{C}-\mathrm{N}$ dimerization.

A

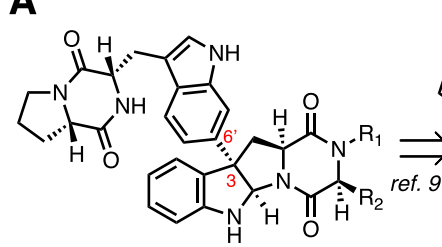

(+)-naseseazine $\mathrm{A}(\mathbf{1}), \mathrm{R}_{1}=\mathrm{H}, \mathrm{R}_{2}=\mathrm{Me}$ (+)-naseseazine $B(2) \mathrm{R}_{1}=\mathrm{R}_{2}=\left(\mathrm{CH}_{2}\right)_{3} \mathrm{CH}_{3}$
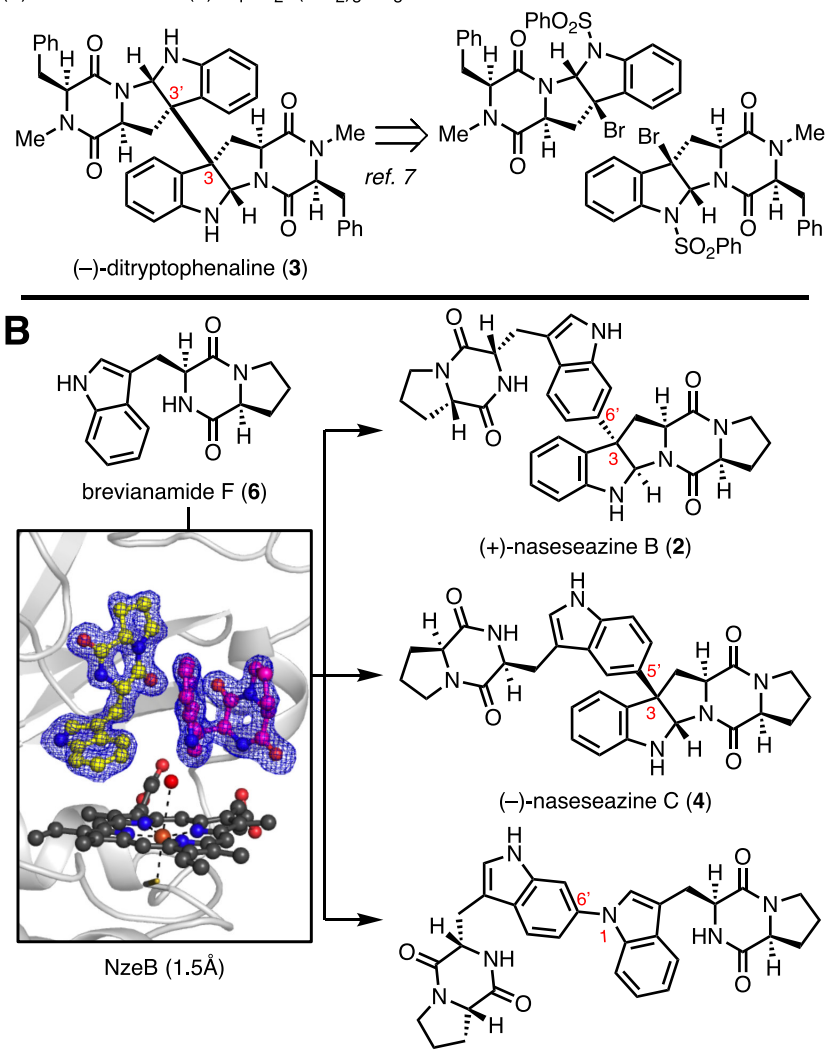

(-)-aspergilazine A (5)

Figure 1. A: Representative examples of substrate controlled, biomimetic total syntheses of dimeric diketopiperazine natural products. B: Current work describing the molecular basis for catalyst-controlled NzeB mediated assembly of isomeric dimers.

Identification of the nzn gene cluster. Previous studies by Tian et al. identified a gene cluster (nasc) from Streptomyces sp. CMBMQ030 composed of a cyclodipeptide synthase (CDPS), nascA, and $\mathrm{P} 450$, nascB, which mediates assembly of cryptic diketopiperazine dimer (-)-naseseazine $C(4) .{ }^{20}$ Our independent sequencing and annotation of the Streptomyces sp. CMB-MQ030 genome (Supplementary Information, Table S1) identified an additional operon, nzn, also composed of a CDPS and P450, each with high sequence homology to nasc counterparts ( NznA/NascB $=61 \%$, $\mathrm{NznB} / \mathrm{NascB}=68 \%)$. Given the high sequence homology to the nasc gene cluster, we hypothesized $\mathrm{P} 450 \mathrm{NznB}$ catalyzes formation of the site- and stereoisomeric (+)-naseseazines A (1) and B (2), the original dimeric DKP natural products isolated from this strain. To assess NznB as the (+)-naseseazines A and B synthase as well as evaluate relative catalytic activities, both recombinant proteins ( $\mathrm{NznB}$ and $\mathrm{NascB}$ ) were subjected to dimerization conditions with predicted native substrate brevianamide $F(6)$. Homodimerization reactions demonstrated $\mathrm{NznB}$ catalyzes formation of 6 into 2, albeit in modest total turnover number (TTN), $145 \mathrm{TTN}$, relative to the conversion of 6 to 4 by NascB, $>500$ TTN (Figure 2). ${ }^{22}$

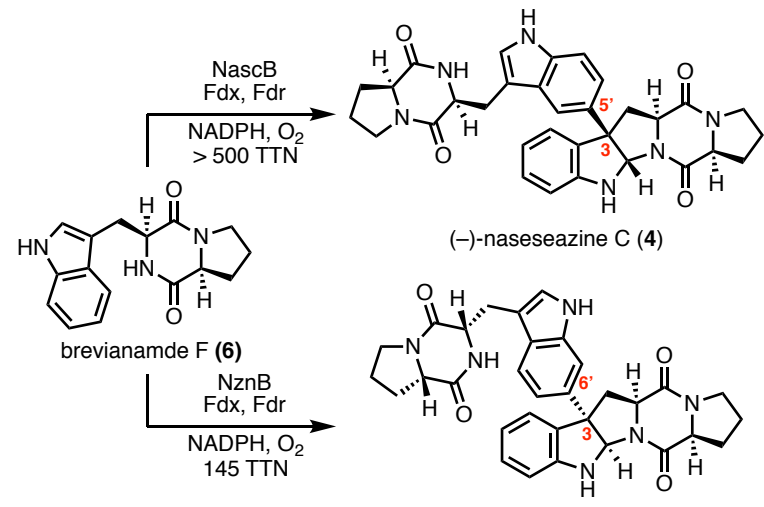

(+)-naseseazine B (2)

Figure 2. Products (-)-naseseazine C (4) and (+)-naseseazine B (2) formed from homodimerizations of 6 catalyzed by NascB and NznB.

To confirm the structure of (-)-naseseazine C (4), as well as resolve a discrepancy in the literature regarding its connectivity, ${ }^{10,11}$ the first total synthesis of (-)-naseseazine C (4) was performed, using the undirected Friedel-Crafts methodology described in the first generation total synthesis of (+)-naseseazines A and B (Scheme 1). ${ }^{9}$ This route was provided dimers with connectivities at both $\mathrm{C} 5$ and $\mathrm{C} 6$, encompassing the constitutional isomers proposed in both isolation reports. Detailed analysis of key HMBC correlations confirmed both the stereochemical configuration of the dimerization axis and connectivity of (-)-naseseazine $C(4)$ at $\mathrm{C} 5$, as well as its $\mathrm{C} 6$ constitutional isomer, which we name (-)isonaseseazine $\mathrm{C}$ (11) based on previous precedent. ${ }^{9}$

Scheme 1 . Total synthesis of (-)-naseseazine $C(4)$ and (-)-iso-naseseazine C (11)
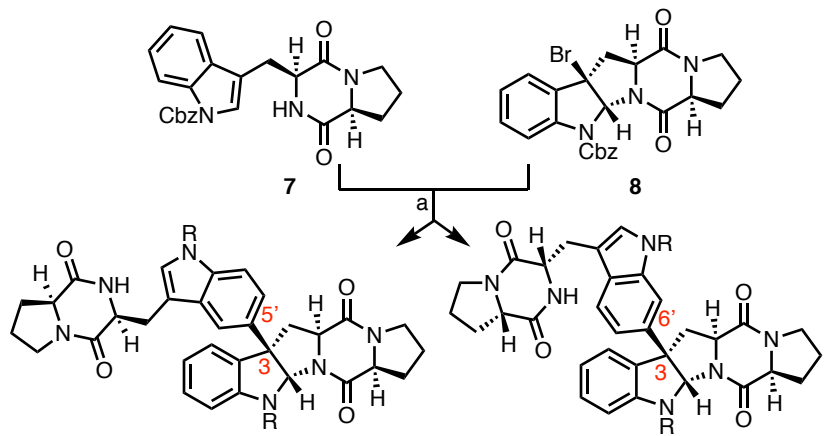

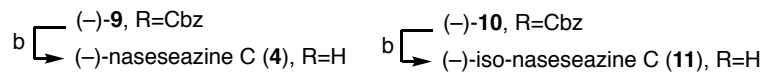

${ }^{a}$ Reagents and conditions: (a), $\mathrm{AgSbF}_{6}, \mathrm{EtNO}_{2},(-)-9:(-)-10=1: 1.4$, $40 \%$. (b) $\mathrm{H}_{2} / \mathrm{Pd} / \mathrm{C}$, EtOH, ( $80 \%$ for (-) -4 and $84 \%$ for (-)- 11 .

Discovery and functional characterization of NzeB. Given the divergent site- and stereoselectivities displayed by $\mathrm{NascB}$ and NznB, we reasoned that comparative sequence analysis may uncover regions conferring selectivity to the dimerization cascade. Therefore, 
we sought to identify additional DKP dimerases through sequence similarity network (SSN) analysis. ${ }^{23}$ Using NascB and NznB as in silico probes, along with known DKP functionalizing P450s from the thaxtomin (12), ${ }^{24}$ pulcherrimic acid (13), ${ }^{25}$ guanitrypmycin (14), ${ }^{26-29}$ bicyclomycin (15), ${ }^{30,31}$ and mycocyclosin $(16)^{32,33}$ BGCs, we mined the NCBI database to identify candidate DKP dimerases. The resulting sequences were assembled into a SSN, which revealed a series of nodes clustered with $\mathrm{NascB}$ and NznB. From these data we identified a BGC, nze, consisting of a CDPS (NzeA) and P450 (NzeB) pair with high sequence homology to the nasc and nzn operons (Figure 3).

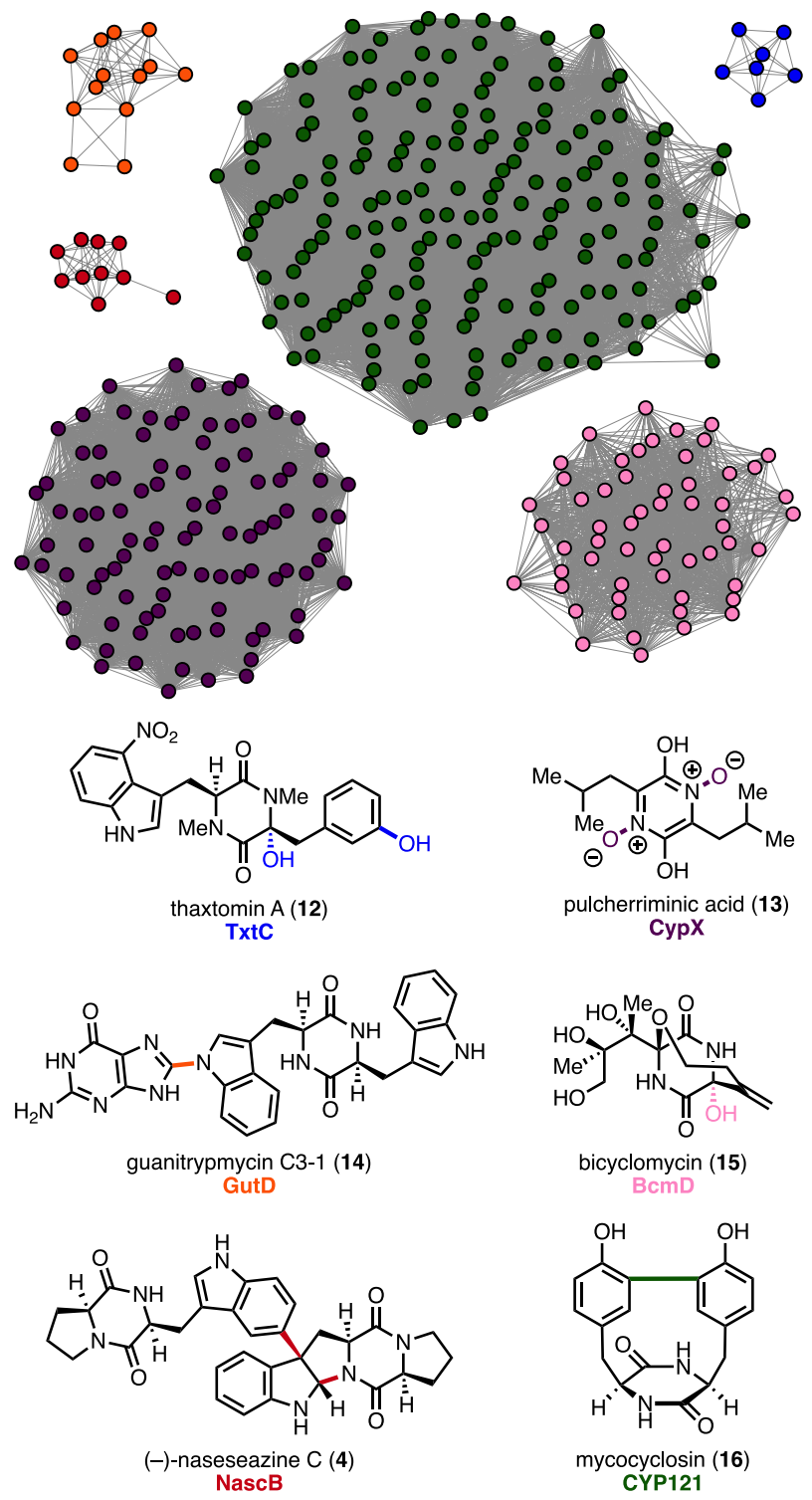

Figure 3. Sequence similarity network with clusters representing characterized DKP functionalizing P450s (clusters of unknown function and orphan nodes omitted for clarity).

Heterologous expression of CDPS, NzeA, and analysis of its product profile identified 6 as the sole DKP metabolite (Supporting Information, Supplementary Figure 8). Subsequent NzeBcatalyzed homodimerization of 6 resulted in formation of three dimeric products; (-)-naseseazine $\mathrm{C}(4)$ as the major product, and two minor products, (+)-naseseazine B (2) and unknown dimer 5 (Figure 4). Scale up and NMR characterization revealed 5 to be $\mathrm{C}-$
$\mathrm{N}$ linked dimer (-)-aspergilazine A. This product distribution exemplifies the versatility of $\mathrm{NzeB}$, as it is able to form site- and stereoisomeric $\mathrm{Csp}^{3}-\mathrm{Csp}^{2}$ linked dimeric products 2 , and 4 , as well as display variant chemoselectivity in the formation of $\mathrm{Csp}^{2}-\mathrm{Nsp}^{2}$ linked 5 (Figure 1b). ${ }^{10,12,14,15}$ The multifunctional nature of NzeB enables assembly of all currently known bacterial-derived DKP dimer scaffolds from a single biocatalyst.
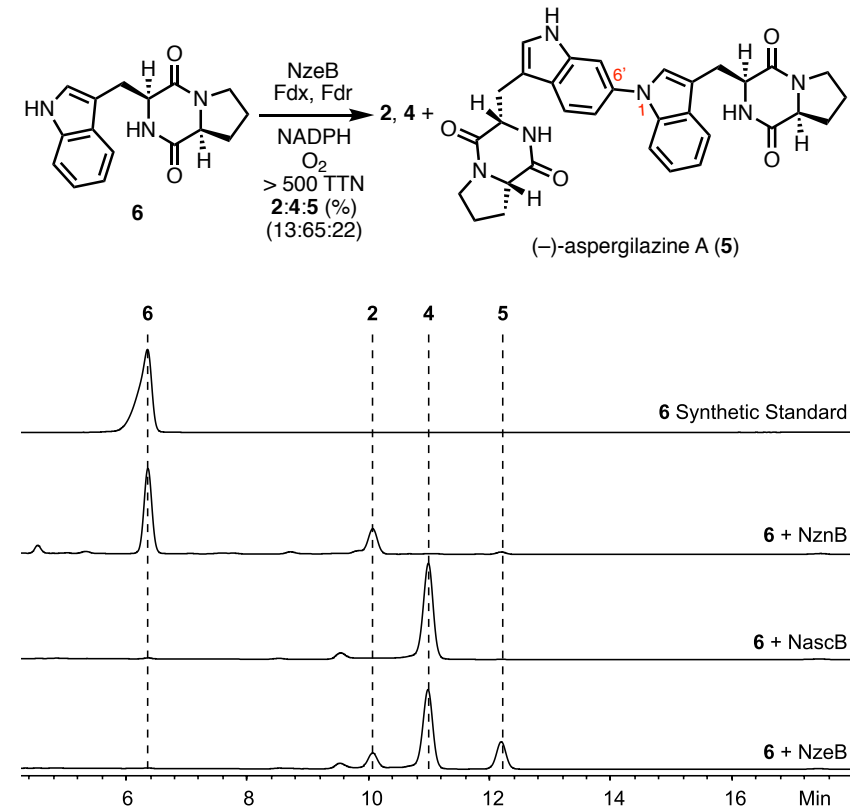

Figure 4. Products formed and HPLC traces from homodimerization of 6 by NzeB.

Crystal structure of NzeB. Given the catalytic flexibility displayed by NzeB, we sought to identify the molecular basis for this versatility using X-ray crystallography. We obtained high-resolution (1.5 Å) crystal structures of NzeB both in the presence and absence of native substrate 6 (Figure 5, Supporting Information Supplementary Figure 21). NzeB shares highest structural homology with DKP functionalizing cytochrome P450, CYP121, which catalyzes oxidative intramolecular $\mathrm{C}-\mathrm{C}$ bond formation to generate mycocyclosin (30\% Sequence ID, $2.4 \AA$ RMSD, 45.8 Z-score). ${ }^{34} \mathrm{NzeB}$ adopts the prototypical triangular prism $\mathrm{P} 450$ fold, with only minor structural rearrangement between the ligand-bound and ligand-free forms (0.45 $\AA$ RMSD). Notably, both ligand-free and substrate bound structures of NzeB display electron density for the conserved axial water $3 \AA$ from the heme-iron.

The active site of the ligand bound crystal structure of NzeB contains two units of 6 , with each substrate occupying a discrete pocket in the active site. The DKP core and tryptophan side chain of the first substrate (Figure 5, light pink) are "sandwiched" by Gln68 and Phe391, forcing the DKP to adopt a concave architecture. The concave pose of this substrate is suggestive of the pyrroloindoline scaffold in the $\mathrm{C}-\mathrm{C}$ linked dimeric products $\mathbf{2}$ and $\mathbf{4}$ and as such we named this site the "cyclization site." The proline side chain of the second substrate (Figure 5, mint green) is encapsulated by predominately hydrophobic residues (Gln75, Glu76, Phe177, Phe390, Phe391) and binds in an extended pose. This extended conformation positions the indole atoms that undergo $\mathrm{C}-\mathrm{H}$ functionalization to form the dimeric axis ( 55 for 4 and $\mathrm{C} 6$ for 2 and 5 ) proximal to the iron center of the heme cofactor; therefore, we named this the "dimerization site." 


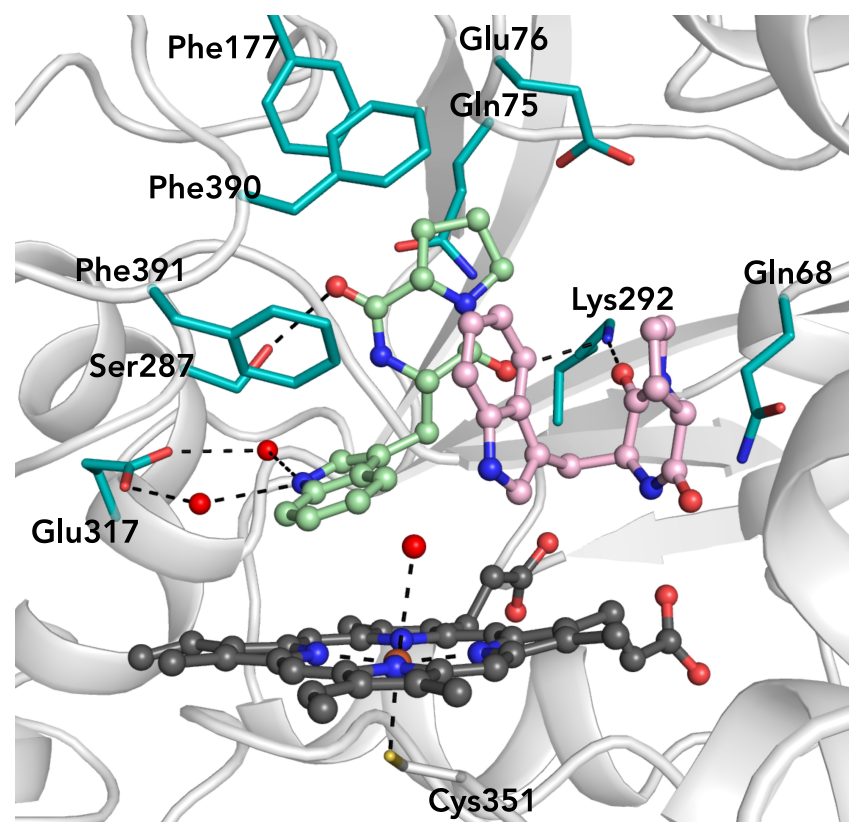

Figure 5. Active site of NzeB with concave substrate (light pink) bound in the "cyclization site" and extended substrate (mint green) bound in the "dimerization site."

Inspection of this complex revealed close packing of the substrate proline side chain in the dimerization site compared to the relatively open cavity surrounding the proline side chain in the cyclization site (Supporting Information, Supplementary Figure 22).

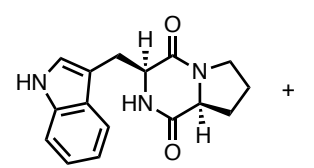

brevianamide $F(6)$
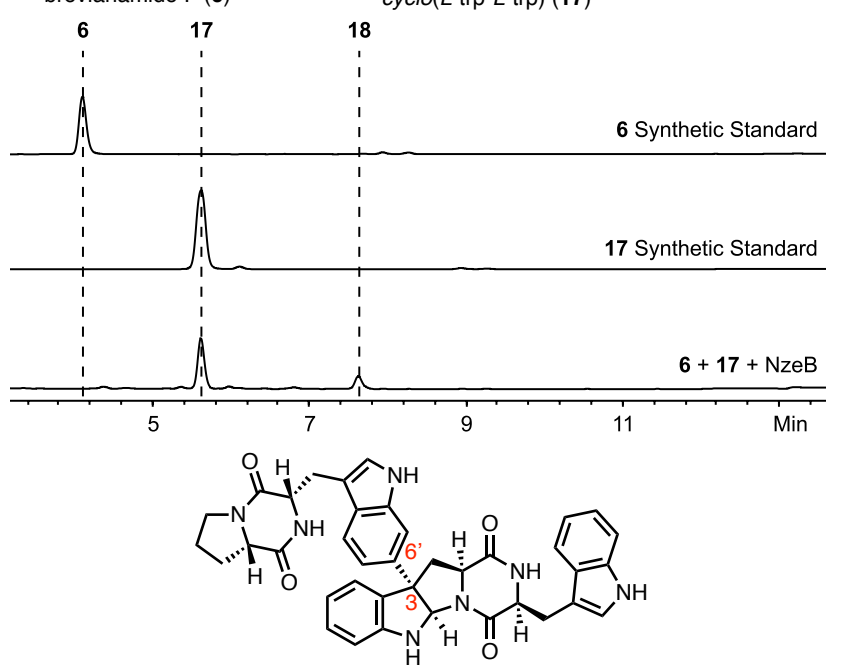

18

Figure 7. Heterodimerization reaction of NzeB with substrates 6 and 17, HPLC traces from heterodimerization reactions and structure of heterodimer 18.

We reasoned that the more open cyclization site could selectively accommodate non-native substrates with bulkier side chains. We hypothesized that reactions involving heterodimerization of the larger substrate and the native DKP 6 would show pyrroloindoline ring formation in the larger substrate due to selective binding in the putative cyclization site.

To confirm the roles of the cyclization and dimerization sites, nonnative DKP substrates with bulky side chains in place of proline were synthesized and screened for their heterodimerization capacity with native substrate 6 (Supporting Information, Supplementary Figure 12). Although a variety of these substrates were capable of forming heterodimers with 6 , only heterodimerizations with $c y$ clo( $L$-Trp- $L$-Trp) (17) resulted in the exclusive formation of a single heterodimeric product (Figure 7). Suppression of homodimeric product formation in the presence of 11 and formation of a single heterodimer suggested that each substrate bound selectively and preferentially to a single DKP binding site.

This biocatalytic union of substrates 6 and 17 was performed on preparative scale and the structure of product 18 was determined. This result suggests that binding of $\mathbf{1 7}$ is limited to the cyclization site. We obtained a crystal structure of a mixed-ligand complex of NzeB with 17 binding in the cyclization site and 6 in the dimerization site, confirming our assignment of the functional roles of each of these active site pockets (Figure 8).

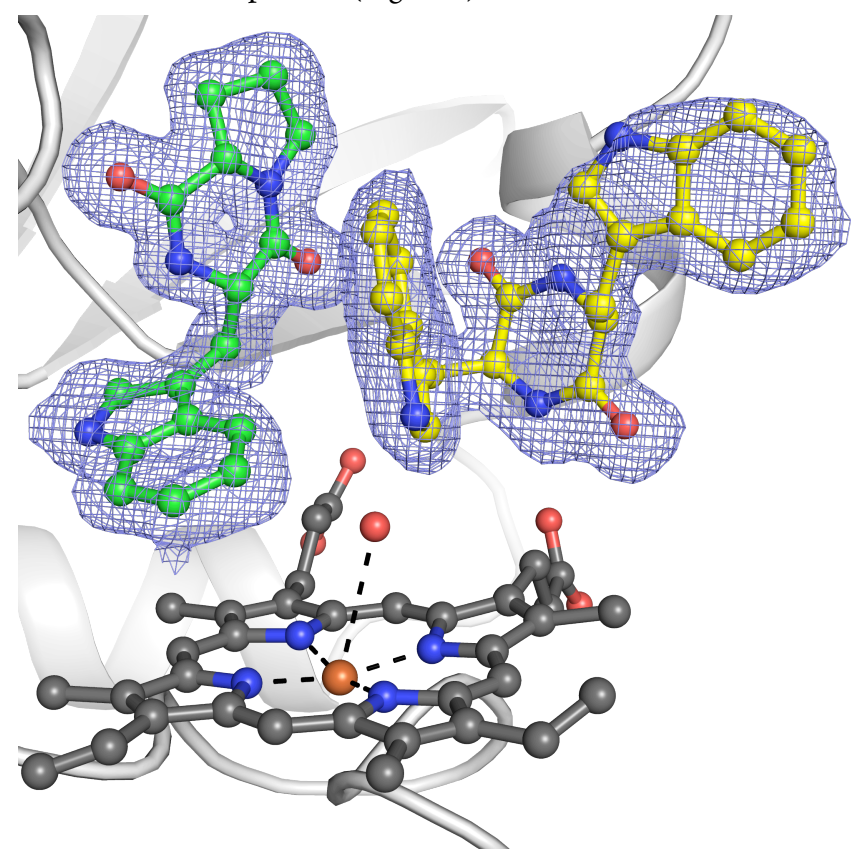

Figure 8. Crystal structure of NzeB heterocomplex with diketopiperazine 17 (yellow) bound in the cyclization site and diketopiperazine 6 (green) bound in the dimerization site, with omit map $\left(\mathrm{F}_{0}-\mathrm{F}_{\mathrm{c}}\right)$ displayed as blue mesh.

Site-directed mutagenesis of NzeB. To identify key residues that control chemoselectivity in the NzeB catalyzed dimerization cascade, we examined the conservation of amino acid residues in both the cyclization and dimerization sites between NzeB, NascB and $\mathrm{NznB}$. As (-)-naseseazine C (4) is the major product of the NzeB and the exclusive product of $\mathrm{NascB}$, we began our analysis by comparing the active sites of these two homologs. A single residue variation exists in the active sites of NascB and NzeB, (NascB: Ala 287, NzeB: Ser 287). In the ligand-bound structures of NzeB, Ser287 forms a hydrogen bond with the proline carbonyl of the substrate in the dimerization site (Figure 5). NzeB variant $\mathrm{NzeB}_{\text {S287 }}$ was generated and homodimerization reactions of 6 with this mutant protein 


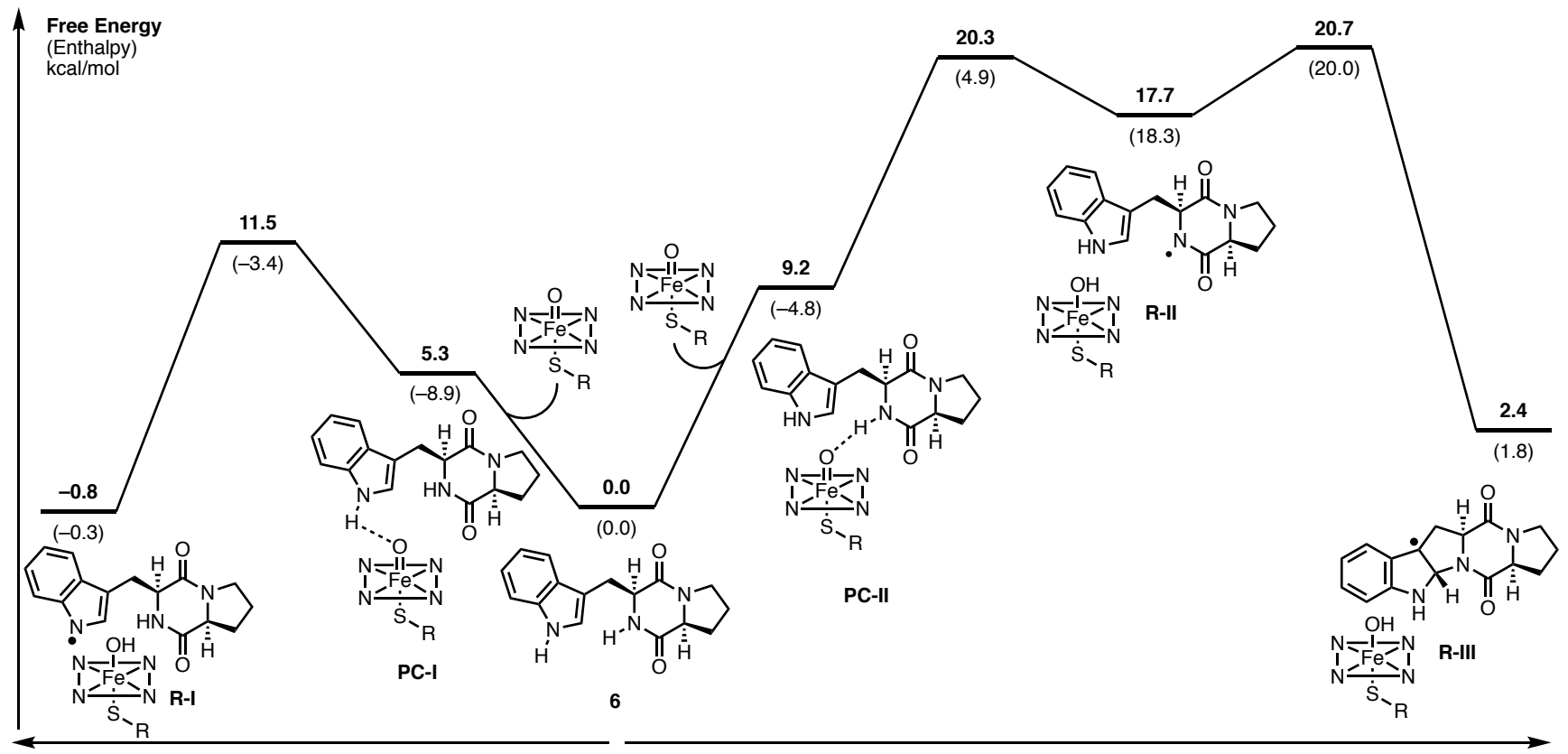

Indole N-H Abstraction

Diketopiperazine N-H Abstraction

Figure 9. Computed mechanistic pathways for the formation of radical cyclized pyrroloindoline intermediate (R-III) via indole N-H abstraction (left) and DKP N-H abstraction (right).

resulted in exclusive formation of (-)-naseseazine C (4) (Figure 10). Functionally, this mutation completely reconstituted the specificity of NascB and pinpointed Ser287 as a key determinant of dimerase selectivity.

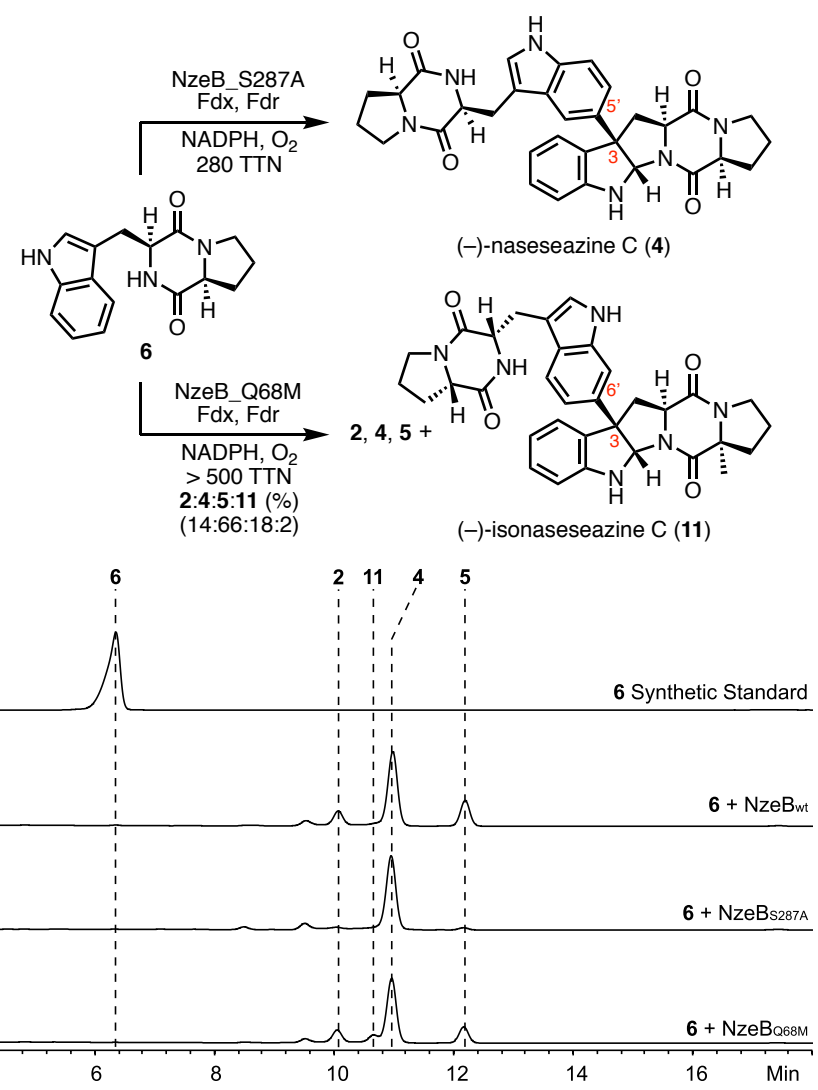

Figure 10. Homodimerization reactions of NzeB variants with 6 and corresponding HPLC traces.
To examine the generality of remodeling active sites to recapitulate selectivity, we compared the active site residues of $(+)$-naseseazine B (2) synthase, NznB, to NzeB. This revealed a single active site variation (NznB: Met68, NzeB: Gln68), located in the cyclization site. To assess the consequence of this variation, $\mathrm{NzeB}_{\mathrm{Q} 68 \mathrm{M}}$ was constructed and subjected to homodimerization reactions with 6 . Rather than exclusive formation of $\mathbf{2}$, homodimerization reactions of 6 with $\mathrm{NzeB}_{\mathrm{Q} 68 \mathrm{M}}$ generated dimers 2, 4 and 5, as well as a new metabolite, 11 (Figure 10). Scale up and characterization of this product revealed its identity as the C6 constitutional isomer of $\mathbf{2}$, (-)-isonaseseazine C (11) based on comparison to synthetic standard (Scheme 1). ${ }^{9}$

To further profile the role of active site residues in controlling selectivity in the $\mathrm{C}-\mathrm{C} / \mathrm{C}-\mathrm{N}$ bond-forming cascade, we performed alanine scanning mutagenesis on residues within $5 \AA$ of either substrate. The resulting variants (S287A, Q75A, E76A, Q75A/E76A, F177A, E317A, F390A, F391A and F390A/F391A) were profiled for catalytic activity and selectivity against native substrate 6 (Supporting Information, Supplementary Figure 13). All functional variants from this experiment displayed the same product profile as $\mathrm{NzeB}_{\mathrm{Q} 68 \mathrm{M}}$, formation of $2,4,5$ and 13 , and differed only in the relative distributions of dimers. Given the increased formation of 13 across a variety of active site variants at various positions within the NzeB active site, we hypothesize that 13 results from loss of selectivity during the dimerization cascade.

NzeB dimerization mechanism and quantum mechanics (QM) calculations. The first biomimetic total synthesis of the (+)naseseazines proposed two alternative biosynthetic mechanisms for DKP dimer formation. ${ }^{9}$ Both mechanisms involved oxidative formation of a pyrroloindoline radical, but diverged on formation of the intermolecular $\mathrm{C}-\mathrm{C}$ bond. The first mechanism proposed directed pyrroloindoline radical coupling to a second DKP followed by oxidative re-aromatization to afford the non-symmetrical DKP scaffold. The second involved oxidation of the pyrroloindoline radical to a tertiary, benzylic cation followed by electrophilic 

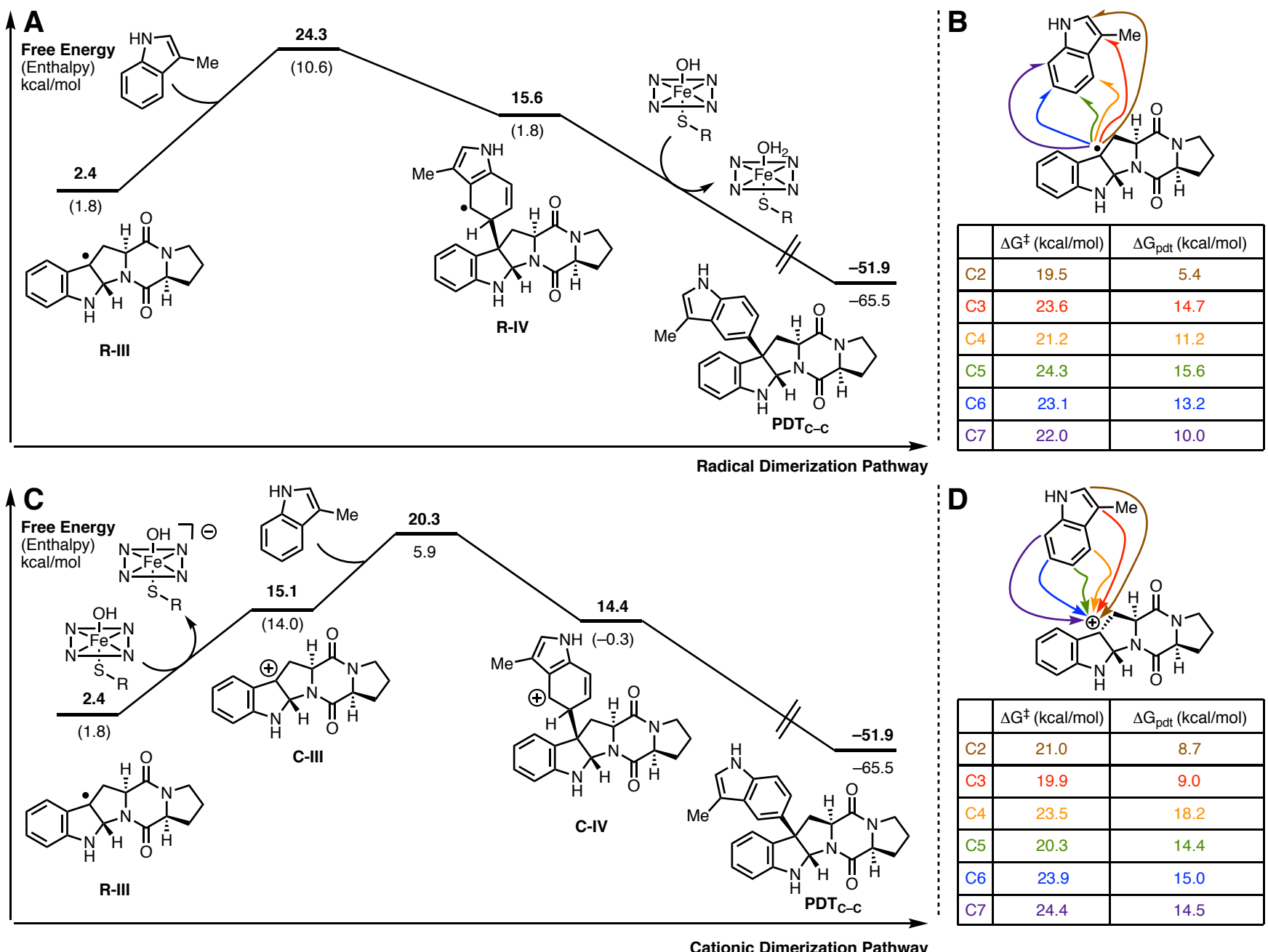

Figure 11. A. Computed radical dimerization pathways to generate (-)-naseseazine $\mathrm{C}_{\text {model }} \mathrm{PDT}_{\mathrm{C}-\mathrm{C}}$ and $\mathrm{B}$ : Transition state and product free energies for both radical $\mathrm{C}-\mathrm{C}$ bond formation at indole positions $\mathrm{C} 2-\mathrm{C} 7$ for $\mathrm{PDT}_{\mathrm{C}-\mathrm{C}}$. C: Computed cationic dimerization pathways to generate $\mathrm{PDT}$ - $\mathrm{C}$ and $\mathrm{D}$ : Transition state and product free energies for cationic $\mathrm{C}-\mathrm{C}$ bond formation at indole positions $\mathrm{C} 2-\mathrm{C} 7$ for $\mathrm{PDT}_{\mathrm{C}-\mathrm{C}}$.

aromatic substitution (EAS) to form the $\mathrm{C}-\mathrm{C}$ bond. The reaction was completed by re-aromatization via deprotonation of the resulting Wheland intermediate to forge the dimeric axis. To shed light on the viability of these proposed mechanisms for intermolecular $\mathrm{C}-\mathrm{C}$ bond formation, as well as the intermolecular $\mathrm{C}-\mathrm{N}$ bond formation to give 5 (catalyzed by NzeB), we turned to quantum mechanical (QM) calculations.

Given the proximity and orientation of the substrate in the cyclization site relative to the heme iron, we propose that the dimerization cascade is initiated by the oxidation of the substrate occupying the cyclization site. The mechanistic pathways (Figure 9) compare two possible modes for oxidative formation of the cyclized pyrroloindoline radical: hydrogen abstraction of 1 ) the proximal indole $\mathrm{N}-\mathrm{H}$ bond or 2) the diketopiperazine $\mathrm{N}-\mathrm{H}$ bond starting from separated brevianamide $\mathrm{F}(6)$ and $\mathrm{P} 450$ iron-oxo (Compound I). While indole $\mathrm{N}-\mathrm{H}$ abstraction has a lower free energy barrier (path $1,11.5$ $\mathrm{kcal} / \mathrm{mol}$ ) than DKP N-H abstraction (path 2, $20.3 \mathrm{kcal} / \mathrm{mol}$ ), both pathways are energetically plausible and subsequent steps have equal or higher free energy barriers. Furthermore, the diketopiperazyl radical (R-II) can cyclize directly to the pyrroloindoline radical (R-III) with a free energy barrier of $20.7 \mathrm{kcal} / \mathrm{mol}$, while cyclization of the indolyl radical (R-I) would need to be accompanied by the net movement of a hydrogen atom from the DKP ni- trogen to the indole nitrogen. A unique feature of the active site of NzeB is the hydrogen bonding of the DKP N-H to a heme propionate. We considered the heme propionate as a possible hydrogen relay to enable cyclization of indolyl radical (R-I), however calculations using acetate as a surrogate for the heme propionate gave a large free energy barrier $(30.8 \mathrm{kcal} / \mathrm{mol})$. Furthermore, there is an overall absence of participant residues (both side chain and backbone atoms) in the active site to catalyze this proton transfer. Single electron oxidation of indole to give a radical carbocation was also considered (Supporting Information, Supplementary Figure 23), however given the high energy radical cation intermediate (41.2 $\mathrm{kcal} / \mathrm{mol}$ ) we did not to pursue this pathway further. In both depicted N-H abstraction pathways, a significant contribution to the overall free energy barrier is the formation of a pre-complex (PC) between the heme iron-oxo species and the substrate $\mathrm{N}-\mathrm{H}$ bond to be abstracted (Figure 9, PC-I and PC-II). Compared to separated starting materials (diketopiperazine 6 and the heme iron-oxo), the free energy for the formation of the $\mathrm{PC}$ for indole $\mathrm{N}-\mathrm{H}$ abstraction (PC-I) and the PC for DKP N-H abstraction (PC-2) are 5.3 $\mathrm{kcal} / \mathrm{mol}$ and $9.2 \mathrm{kcal} / \mathrm{mol}$, respectively. However, as evidenced by the crystal structure of NzeB, both DKP substrates are oriented to facilitate PC formation substantially lowering the free 

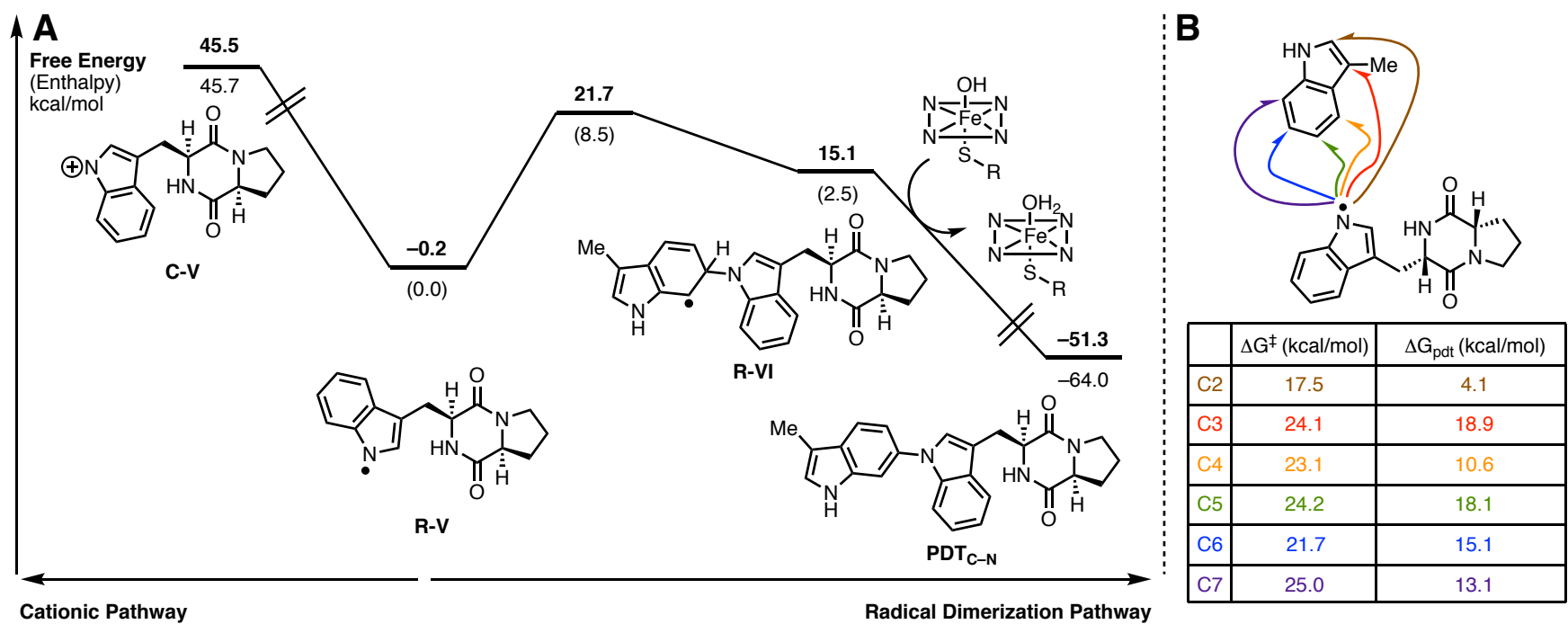

Figure 12. A. Computed dimerization pathways for the formation of (-)-aspergilazine A (5): radical dimerization pathway and demonstration that a cationic pathway is thermodynamically unfavorable. B. Transition state and product free energies for radical C-N bond formation at indole positions C2-C7 for (-)-aspergilazine A (5) model PDT C-N. $_{\text {. }}$

energy barrier for the entire transformation.

Following cyclization to the pyrroloindoline radical (Figure 11, RIII), formation of the $\mathrm{C}-\mathrm{C}$ bond with a second unit of 6 (modeled as 3-methylindole) may occur through either a radical-mediated, or a cationic Friedel-Crafts mechanism. The depicted energetic landscape of both dimerization mechanisms lead to formation of (-)naseseazine C (4) (Figure 11), however, analogous computations of the pathway leading to the formation of (+)-naseseazine B (2) (Supporting Information), were qualitatively similar and led to the same conclusions. In the radical-mediated mechanism, $\mathrm{C}-\mathrm{C}$ bond formation is the rate-determining step with a free energy barrier of $24.3 \mathrm{kcal} / \mathrm{mol}$, while in the cationic mechanism the $\mathrm{C}-\mathrm{C}$ bond formation barrier of $20.3 \mathrm{kcal} / \mathrm{mol}$ falls slightly below the preceding cyclization. Both mechanisms remain viable given that these computations are performed on a model system in the absence of active site residues, and that the computed barriers are relatively close. However, NzeB lacks any side chains, backbone amides, or water molecules at an appropriate location in the active site to act as a general base for deprotonation of Wheland intermediate (C-IV) generated in the cationic Friedel-Crafts pathway. Furthermore, alanine mutants of distal acidic/basic residues in the active site (Q78, Q75, E76, E317), which could act to re-aromatize the Wheland intermediate (C-IV) are still catalytically competent for dimerization (Supporting Information, Supplementary Figure S13). However, re-aromatization of intermediate (R-IV) via $\mathrm{H}$ atom abstraction by the iron(IV)-hydroxo (Compound II) is likely to be facile given the proximity of $\mathrm{C} 5$ and $\mathrm{C} 6$ to the heme co-factor. Alternatively, localization of $\mathrm{C} 5$ and $\mathrm{C} 6$ to the heme may also allow a single electron oxidation of intermediate R-IV to intermediate C-IV by Compound II followed by deprotonation and rearomatization of C-IV by the resulting iron(III)-hydroxo species.

In addition to catalyzing intermolecular $\mathrm{C}-\mathrm{C}$ bond formation, $\mathrm{NzeB}$ also catalyzes the intermolecular $\mathrm{C}-\mathrm{N}$ bond formation to generate (-)-aspergilazine A (5). Given the viability of both a radical and cationic dimerization mechanism for NzeB catalyzed $\mathrm{C}-\mathrm{C}$ bond formation, we also compared the energetic landscapes of radical and cationic pathways for $\mathrm{C}-\mathrm{N}$ bond formation starting from the uncyclized indolyl radical (Figure 11). In the radical- mediated pathway, the rate-limiting $\mathrm{C}-\mathrm{N}$ bond formation has a thermodynamically plausible free energy barrier of $21.7 \mathrm{kcal} / \mathrm{mol}$. In contrast, a potential cationic pathway would require oxidizing the indolyl radical $(\mathrm{R}-\mathrm{V})$ to a cation $(\mathrm{C}-\mathrm{V})$, which is thermodynamically demanding $(45.5 \mathrm{kcal} / \mathrm{mol})$. Given that the oxidation needed to access a cationic pathway is so highly unfavorable, quantum mechanical computations clearly support a radical-mediated pathway in the case of $\mathrm{C}-\mathrm{N}$ bond formation.

The $\mathrm{C}-\mathrm{C}$ bond forming cascade catalyzed by NzeB results in the formation of two $\mathrm{C}-\mathrm{C}$ linked dimers 2 and 4 , as well as the $\mathrm{C}-\mathrm{N}$ bond linked dimer 5 . Computations were performed to examine the innate selectivity for cation and radical mechanisms for intermolecular $\mathrm{C}-\mathrm{C}$ bond formation (Figure 11B \& 11D), and analogous computations were performed for radical $\mathrm{C}-\mathrm{N}$ bond formation (Figure 12B). For all computations relating to $\mathrm{C}-\mathrm{C}$ and $\mathrm{C}-$ $\mathrm{N}$ bond formation, regardless of radical or cation mechanism, the observed dimerization site selectivity displayed by NzeB does not coincide with either the transition states or products that are lowest in free energy. Thus, these computations suggest that DKPdimerization is catalyst-controlled rather than driven by the innate reactivity of the DKP substrates, with $\mathrm{NzeB}$ guiding the selective intermolecular $\mathrm{C}-\mathrm{C}$ and intramolecular $\mathrm{C}-\mathrm{N}$ bond formation.

Discussion

Biosynthesis of the tryptophan-linked dimeric DKP alkaloids reveal Nature's ability to build molecular complexity from simple and abundant building blocks captured from primary metabolism. The production of DKP dimers from both fungal and bacterial origin indicates the biological utility of this natural product scaffold. Characterized fungal systems utilize non-ribosomal peptide synthetases (NRPS) to generate DKP monomers, while bacterial pathways generate the same monomers via CDPSs. Characterization of CDPS-associated gene clusters has decrypted the biosynthetic pathways for known natural products such as bicyclomycin, ${ }^{30,} 31$ albonoursin, ${ }^{35}$ and the drimentines. ${ }^{36}$ Furthermore, these investigations have also identified new classes of natural products as well as unique DKP tailoring reactions including dehydrogenation, ${ }^{35} \mathrm{C}-\mathrm{H}$ hydroxylation, ${ }^{30,}{ }^{31} \mathrm{C}-$ - $\mathrm{O}_{-}$, and $\mathrm{N}-$ methylation, ${ }^{37}$ prenylation and cyclization of terpenes, ${ }^{36}$ and oxidative coupling with purine nucle- 
obases. $^{26-29}$ P450-mediated diketo-piperazine dimerization further expands the repertoire of tailoring events found in CDPSassociated pathways.

The isolation of isomeric DKP dimers from a single organism is relatively common, which suggested two biosynthetic postulates; 1) a single dimerase with low selectivity enabled the assembly of isomeric products, or 2) a discrete dimerase was required for each coupling mode. Our results demonstrate there is no general axiom for bacterial biosynthesis of DKP dimers. P450s NascB and NznB from Streptomyces sp. CMB-MQ030 each catalyze a site- and stereoselective dimerization of 6 resulting in (-)-naseseazine $C$ (4) and (+)-naseseazine B (2), respectively. By contrast, NzeB catalyzes both known bacterial $\mathrm{C}-\mathrm{C}$ bond forming dimerization modes generating 2 and 4 , while also displaying chemodivergent reactivity in the formation of $\mathrm{C}-\mathrm{N}$ bond linked dimer, (-)-aspergilazine $\mathrm{A}$ (5). While "multifunctional" bacterial P450s are well known, ${ }^{38}$ this typically refers to the ability for a P450 to perform multiple monooxygenase reactions, such as hydroxylations at multiple sites, ${ }^{39}$ hydroxylation and epoxidation activity ${ }^{40}$ hydroxylation with continued reaction to higher oxidation states, or a combination of these processes. NzeB differs from these enzymes in its ability to mediate formation of site- and stereoisomeric $\mathrm{C}-\mathrm{C}$ linked products $\mathbf{1}$ and 2 , and also catalyzes direct $\mathrm{Csp}^{2}-\mathrm{H}$ amination in the formation of $\mathbf{5}$. Recently, multiple reports have disclosed engineered enzymes capable of performing inter- and intramolecular $\mathrm{C}-\mathrm{H}$ amination. ${ }^{41,}{ }^{42}$ However this transformation is relatively rare in nature with few examples in the literature, ${ }^{43,44}$ and to our knowledge, NzeB represents the first structurally characterized, wild-type $\mathrm{P} 450$ to catalyze intermolecular $\mathrm{Csp}^{2}-\mathrm{H}$ amination.

We were able to obtain multiple high-resolution crystal structures, including NzeB ligand free, and in complex with select native and non-native substrates. Our biosynthetic hypotheses for the $\mathrm{C}-\mathrm{C}$ bonded dimers 2 and 4 were supported by direct observation of the NzeB-DKP complex, with the enzyme possessing two distinct binding sites to pre-organize substrates for diastereoselective cyclization as well as site-selective $\mathrm{C}-\mathrm{C}$ bond formation. Synthesis and screening of non-native substrates for their selective heterodimerization enabled validation of the cyclization and dimerization sites. Moreover, we were able to capture a complex of NzeB with sterically differentiated ligands, brevianamide $\mathrm{F}(6)$ and $c y c l o(L$-Trp- $L$-Trp) (17), which unambiguously confirmed our assignment of roles to each DKP binding site. The connectivity and configuration of the dimerization axis of heterodimeric product 18 is identical to that of the NzeB minor product (+)-naseseazine B (2), and is consistent with the binding mode of the two monomeric precursors (Figure 8 ). We hypothesize this is due to the markedly larger side chain of 17 which occupies the additional channel in the cyclization site wherein the native substrates may occupy during catalysis in order for both substrate and enzyme to undergo the conformational changes necessary to access either the (-)-naseseazine $C$ or (-)aspergilazine A scaffolds. Notably, all other structurally characterized DKP functionalizing CYP450s (CYP121, ${ }^{33} \mathrm{CypX}^{25}$ and $\mathrm{TxtC}^{24}$ ) bind DKPs in the analogous position of the active site, which corresponds to the cyclization site of NzeB. The dimerization site of NzeB is a previously unobserved pocket for DKPs in P450s and accounts for an approximate doubling of the active site volume of $\mathrm{NzeB}\left(832 \AA^{3}\right)$ relative to other DKP binding CYP450s. ${ }^{45}$ Previous efforts in the characterization of NascB demonstrated that in heterodimerization reactions the bulky substrates undergo cyclization to form pyrroloindoline functionality. ${ }^{20}$
Our structural investigations of $\mathrm{NzeB}$ and assignment of the role of the dimerization site provides a structural basis for this phenomenon. Furthermore, identifying the sidechains in the dimerization site that encapsulate the proline moiety of its substrate paves the way to reshaping this binding pocket. Engineering DKP dimerases to enable coupling of DKPs with larger side chains may enable formation of products similar to those isolated from fungi. ${ }^{46-48}$

Mutagenesis of the active site of NzeB provided further insight into how NzeB guides the site- and stereoselective formation of $\mathrm{C}-\mathrm{C}$ and $\mathrm{C}-\mathrm{N}$ bonds. Comparison of the active site residues of $\mathrm{NzeB}$ to NascB and NznB, identified that each possessed a single, active site variation (NzeB: Ser287, NascB: Ala287). To examine the role of this active site variation in selectivity the $\mathrm{NzeB}_{\$ 287 \mathrm{~A}}$ variant was generated and homodimerization reactions with native substrate 6 , $\mathrm{NzeB}_{5287 \mathrm{~A}}$ exclusively generates (-)-naseseazine $\mathrm{C}(4)$ completely reconstituting the selectivity of $\mathrm{NascB}$ with a single substitution. Given this result, we reasoned that through homologous substitution of the active site variation between $\mathrm{NznB}$ and $\mathrm{NzeB}$, located in the cyclization site (NzeB: Gln68 vs NznB: Met68) would provide access to a surrogate of NznB. The prospect of engineering the dimerization selectivity of $\mathrm{NzeB}$ to recapitulate that of $\mathrm{NznB}$ is particularly attractive, as wildtype $\mathrm{NznB}$ has low TTN relative to $\mathrm{NascB}$ and NzeB (Figure 2). However, counter to the results from $\mathrm{NzeB}_{\text {s287A }}$, dimerization reactions with $\mathrm{NzeB}_{\mathrm{Q} 68 \mathrm{M}}$ did not recapitulate the observed $\mathrm{NznB}$ selectivity, rather led to the formation of previously unobserved dimer (-)-isonaseseazine C (11).

These results demonstrate that the selectivity of a given DKP dimerase cannot be reconstituted simply by interchanging active site residues, and therefore the selectivity of $\mathrm{NzeB}$ and its homologues must also be determined by residues outside the active site motif. Thus, we propose that the observed crystal structure represents one of many catalytically relevant conformations, and that the active site must undergo conformational remodeling during catalysis, with the selectivity of the dimerization reaction being influenced by a combination of residues both inside and outside of the active site. This is best represented by the discrepancy between the conformation of substrates in the ligand bound crystal structure of NzeB and the observed product distribution from the dimerization reaction. In the ligand-bound structure of $\mathrm{NzeB}$, both substrates are apparently pre-organized toward formation of $(+)$-naseseazine $\mathrm{B}$ (2), despite this dimer being a minor product. To access conformations that would lead to the major product, diketopiperazine dimer 4, the orientation of substrate in the cyclization site would require inversion from a concave to convex posture. Furthermore, in spin-shift binding assays of $\mathrm{NzeB},{ }^{49}$ substrate 6 was able to displace the water molecules axial to the heme to induce a low- to high-spin transition of the heme iron indicative of direct binding to the active site (Supporting Information, Supplementary Figure 10). However, this axial water is present in both the ligand-bound and ligand-free crystal structures at high-occupancy, suggesting that solution-phase dynamics provide access to currently unobserved conformations. In addition, it is well-precedented that binding of electron transport proteins alters the architecture of cytochromes P450 during catalysis. ${ }^{50}$ Given these factors we propose residues and regions distal to the active site are influencing selectivity and catalysis, and efforts to identify them in bacterial DKP dimerases are currently underway.

Finally, quantum mechanical computations provided new insights into NzeB's ability to catalyze formation of $\mathrm{C}-\mathrm{C}$ and $\mathrm{C}-\mathrm{N}$ bonds. Relative to $\mathrm{C}-\mathrm{O}$ bond forming $\mathrm{P} 450 \mathrm{~s}$, far fewer $\mathrm{C}-\mathrm{C}$ bond forming 
P450s have been structurally characterized; examples include both intramolecular such as StaP (PDB: 2Z3U) ${ }^{51}$ and CYP121 (PDB: $3 \mathrm{G} 5 \mathrm{H}),{ }^{33}$ as well as intermolecular such as CYP158A1/CYP158A2 (PDB: 1T93, 2D09), ${ }^{52,}$ and recently the intramolecular $\mathrm{C}-\mathrm{N}$ bond forming indolactam cytochrome P450 (PDB: 6J82). ${ }^{54} \mathrm{~A}$ common feature among the characterized intra-and intermolecular $\mathrm{C}-\mathrm{C} / \mathrm{C}-\mathrm{N}$ bond forming $\mathrm{P} 450$ s is that one of the reactive atoms participating in $\mathrm{C}-\mathrm{C} / \mathrm{C}-\mathrm{N}$ coupling is proximal to the heme iron while the second reactive atom is distal, and oxidation of this distal atom is facilitated by a basic amino acid residue or water via protoncoupled electron transfer (PCET). ${ }^{55,56}$ However, unique to the structure of $\mathrm{NzeB}$, reactive atoms from both ligands are positioned within $4 \AA$ of the axial water ligand, indicating the possibility for direct oxidation of both ligands catalyzed by the heme without intervention of neighboring residues or solvent (Figure 5). Regardless of mechanism, they indicate that a major role of NzeB is to orient substrates for reaction with the heme iron oxo species. By facilitating the formation of a pre-complex, NzeB can reduce the free energy barrier for the overall transformation by up to 5 to 10 $\mathrm{kcal} / \mathrm{mol}$. Previous investigations into the mechanism for $\mathrm{C}-\mathrm{C}$ bond formation excluded a cationic mechanism due to reactivity of electron poor substrates. ${ }^{20}$ However, the utilization of electronically differentiated substrates for mechanistic studies such as Hammett plots is well represented in the literature, including enzymatic electrophilic aromatic substitution (EAS) type reactions. ${ }^{57}$ Our computations demonstrate that both radical-mediated and cationic pathways are thermodynamically plausible for the formation of $\mathrm{C}-\mathrm{C}$ bonds, with rate-determining transition states of comparable free energies. While there are residues present in the active site that could be positioned for deprotonation of Wheland intermediates, their distance appears too great from the appropriate carbon atoms, and mutation of these residues to alanine does not prevent dimerization. Given the lack of residues or active site water molecules necessary to deprotonate Wheland intermediates generated from a cationic pathway dimerization, and the localization of reactive atoms from both substrates proximal to the heme iron, we propose that $\mathrm{C}-\mathrm{C}$ bond forming dimerization modes likely also proceed via a radical-mediated mechanism. However, re-aromatization of the $\mathrm{C}-\mathrm{C}$ bond formed radical intermediate may occur through either concerted HAT or stepwise PCET by Compound II. In contrast, computations relating to formation of the intermolecular $\mathrm{C}-\mathrm{N}$ bond in (-)-aspergilazine $\mathrm{A}(5)$ demonstrate that the cationic pathway is thermodynamically prohibitive and the radicalmediated mechanism is strongly favored. To examine the site selectivity of $\mathrm{NzeB}$ we also explored computationally the innate selectivity for $\mathrm{C}-\mathrm{C}$ and $\mathrm{C}-\mathrm{N}$ bond forming dimerizations. These computations demonstrate that regardless of cationic or radical mechanism, NzeB overrides the innate selectivity for $\mathrm{C}-\mathrm{C}$ and $\mathrm{C}-\mathrm{N}$ bond formation and the connectivity of the product dimers 2,4 , and 5 are the result of a catalyst-controlled dimerization, rather than the reactivity of the diketopiperazine substrates themselves. Further analysis is required to unambiguously exclude other mechanisms for $\mathrm{C}-$ $\mathrm{C}$ and $\mathrm{C}-\mathrm{N}$ bond formation and is currently underway.

\section{Conclusion}

In conclusion, we identified and characterized the chemically versatile cytochrome P450, NzeB, capable of generating both (+)- and $(-)$-naseseazine frameworks as well as the non-cyclized Csp ${ }^{2}-\mathrm{Nsp}^{2}$ linked (-)-aspergilazine dimeric scaffold. To gain insight into the molecular basis for its versatility, we obtained the first high- resolution crystal structure of a DKP dimerizing P450 in complex with select native and non-native substrates. This structural information revealed a previously unrecognized DKP binding site compared to other reported crystal structures of DKP functionalizing $\mathrm{P} 450 \mathrm{~s}^{24,25,33} \mathrm{NzeB}$ is distinguished from the flaviolin dimerase, in its relatively close binding of DKP substrates proximal (within $4 \AA$ ) to the heme cofactor. Site directed mutagenesis of NzeB demonstrated that active site residues are not the sole drivers of site-, stereo- and chemoselectivity. Tandem structural, biochemical, and computational approaches explored the viability of cationic and radical mechanisms in intermolecular $\mathrm{C}-\mathrm{C}$ bond formation, and provided evidence for a likely radical mechanism for intermolecular $\mathrm{C}-\mathrm{N}$ bond formation. The structural, biochemical, and computational characterization of $\mathrm{NzeB}$ provides the first insight into the molecular basis for DKP dimerization. This new information opens the door to engineering NzeB and its functional homologs for improved total turnover number, broadened substrate scope, and new non-natural dimerization modes furthering our efforts towards a catalyst controlled method for DKP dimerization.

\section{ASSOCIATED CONTENT}

\section{Supporting Information}

The Supporting Information is available free of charge on the ACS Publications website.

Full experimental and computational details, NMR spectra, tables, and figures. (PDF)

\section{AUTHOR INFORMATION}

\section{Corresponding Authors}

Mohammad Movassaghi - Massachusetts Institute of Technology, Cambridge, Massachusetts; orcid.org/0000-0003-3080-1063; Email: movassag@mit.edu

K. N. Houk - University of California, Los Angeles, California; orcid.org/0000-0002-8387-5261; Email: houk@chem.ucla.edu

David H. Sherman - University of Michigan, Ann Arbor, Michigan; orcid.org/0000-0001-8334-3647; Email: davidhs@umich.edu

\section{Other Authors}

Vikram V. Shende - University of Michigan, Ann Arbor, Michigan; orcid.org/ 0000-0001-8396-6297; Email: vvshende@umich.edu

Yogan Khatri - University of Michigan, Ann Arbor, Michigan; orcid.org/0000-0002-2432-7679; Email: ykhatri@umich.edu

Sean A. Newmister - University of Michigan, Ann Arbor, Michigan; orcid.org/ 0000-0002-9623-7779; Email: snewmist@umich.edu

Jacob N. Sanders - University of California, Los Angeles, California; 0000-0002-2196-4234; Email: jacosand@chem.ucla.edu

Petra Lindovska - Massachusetts Institute of Technology, Cambridge, Massachusetts; orcid.org/ 0000-0003-2655-8566. Email: lindovska@alum.mit.edu

Fengan Yu- University of Michigan, Ann Arbor, Michigan; orcid.org/ 0000-0002-4522-349X; Email: fengenyu@umich.edu 
Tyler J. Doyon- University of Michigan, Ann Arbor, Michigan; orcid.org/0000-0002-6885-7487; Email: tdoyon@umich.edu

Justin Kim- Dana-Farber Cancer Institute, Boston, Massachusetts; orcid.org/ 0000-0002-4076-2005; Email: justin.kim50@gmail.com

\section{Author Contributions}

All authors have given approval to the final version of the manuscript.

\section{Notes}

The authors declare no competing financial interest.

\section{ACKNOWLEDGMENTS}

The authors thank the NSF CCI Center for Selective C-H Functionalization (CHE-1700982), NIH grant R35 GM118101 and the Hans W. Vahlteich Professorship (to D.H.S.). M.M. also acknowledges financial support by NIH grant GM089732. We are grateful to Dr. Robert J. Capon for providing Streptomyces sp. CMB MQ-030 under a materials transfer agreement. J.N.S. acknowledges support from the National Institutes of Health for award F32 GM122218. Computational resources were provided by the UCLA Institute for Digital Research and Education (IDRE) and by the San Diego Supercomputing Center (SDSC) through XSEDE (ACI-1548562).

\section{REFERENCES}

1. Hino, T.; Nakagawa, M., Chemistry and Reactions of Cyclic Tautomers of Tryptamines and Tryptophans. The Alkaloids: Chemistry and Pharmacology, Brossi, A., Ed. Academic Press: New York, 1989; Vol. 34, pp 1-75.

2. Anthoni, U.; Christophersen, C.; Nielsen, P. H., Naturally Occurring Cyclotryptophans and Cyclotryptamines. Alkaloids: Chemical and Biological Perspectives, Pelletier, S. W., Ed. Pergamon: London, 1999; Vol. 13, pp 163-236.

3. Borthwick, A. D., 2,5-Diketopiperazines: synthesis, reactions, medicinal chemistry, and bioactive natural products. Chem. Rev. 2012, 112 (7), 3641-716.

4. Tadano, S.; Ishikawa, H., Synthesis of Tryptophan-Based Dimeric Diketopiperazine Alkaloids Using Bioinspired Reactions. Synlett 2014, 25(2), 157-162.

5. Kim, J.; Movassaghi, M., Biogenetically-inspired total synthesis of epidithiodiketopiperazines and related alkaloids. Acc. Chem. Res. 2015, $48(4), 1159-71$.

6. Springer, J. P.; Bűchi, G.; Kobbe, B.; Demain, A. L.; Clardy, J., The structure of ditryptophenaline - a new metabolite of. Tet. Lett. 1977, 18 (28), 2403-2406.

7. Movassaghi, M.; Schmidt, M. A.; Ashenhurst, J. A., Concise total synthesis of (+)-WIN 64821 and (-)-ditryptophenaline. Angew. Chem. Int. Ed. Engl. 2008, 47 (8), 1485-7.

8. Raju, R.; Piggott, A. M.; Conte, M.; Aalbersberg, W. G.; Feussner, K.; Capon, R. J., Naseseazines A and B: a new dimeric diketopiperazine framework from a marine-derived actinomycete, Streptomyces sp. Org. Lett. 2009, 11 (17), 3862-5.

9. Kim, J.; Movassaghi, M., Concise total synthesis and stereochemical revision of (+)-naseseazines $\mathrm{A}$ and $\mathrm{B}$ : regioselective arylative dimerization of diketopiperazine alkaloids. J. Am. Chem. Soc. 2011, 133(38), 14940-3.

10. Xiong, Z. Q.; Liu, Q. X.; Pan, Z. L.; Zhao, N.; Feng, Z. X.; Wang, Y., Diversity and bioprospecting of culturable actinomycetes from marine sediment of the Yellow Sea, China. Arch. Microbiol. 2015, 197 (2), 299-309.
11. Buedenbender, L.; Grkovic, T.; Duffy, S.; Kurtboke, D. I.; Avery, V. M.; Carroll, A. R., Naseseazine C, a new anti-plasmodial dimeric diketopiperazine from a marine sediment derived Streptomyces sp. Tet. Lett. 2016, 57 (52), 5893-5895.

12. Cai, S. X.; Kong, X. L.; Wang, W.; Zhou, H. N.; Zhu, T. J.; Li, D. H.; Gu, Q. Q., Aspergilazine A, a diketopiperazine dimer with a rare N-1 to C-6 linkage, from a marine-derived fungus Aspergillus taichungensis. Tet. Lett. 2012, 53(21), 2615-2617.

13. Kieffer, M. E.; Chuang, K. V.; Reisman, S. E., Copper-catalyzed diastereoselective arylation of tryptophan derivatives: total synthesis of (+)-naseseazines A and B. J. Am. Chem. Soc. 2013, 135 (15), 5557-60.

14. Boyd, E. M.; Sperry, J., Total synthesis of (-)-aspergilazine A. Org. Lett. 2014, 16(19), 5056-9.

15. Chuang, K. V.; Kieffer, M. E.; Reisman, S. E., A Mild and General Larock Indolization Protocol for the Preparation of Unnatural Tryptophans. Org. Lett. 2016, 18 (18), 4750-3.

16. Alonso, I.; Alvarez, R.; de Lera, Á. R., Indole-Indole Ullmann Cross-Coupling for CAr-N Bond Formation: Total Synthesis of (-)Aspergilazine A. Eur.J. Org. Chem. 2017, 2017 (33), 4948-4954.

17. Loach, R. P.; Fenton, O. S.; Movassaghi, M., Concise Total Synthesis of (+)-Asperazine, (+)-Pestalazine A, and (+)-iso-Pestalazine A. Structure Revision of (+)-Pestalazine A. J. Am. Chem. Soc. 2016, 138 (3), 1057-64.

18. Nelson, B. M.; Loach, R. P.; Schiesser, S.; Movassaghi, M., Concise total synthesis of $(+)$-asperazine A and (+)-pestalazine B. Org. Biomol. Chem. 2018, 16(2), 202-207.

19. Saruwatari, T.; Yagishita, F.; Mino, T.; Noguchi, H.; Hotta, $\mathrm{K}$; Watanabe, K., Cytochrome $\mathrm{P} 450$ as dimerization catalyst in diketopiperazine alkaloid biosynthesis. Chembiochem 2014, 15 (5), 6569.

20. Tian, W.; Sun, C.; Zheng, M.; Harmer, J. R.; Yu, M.; Zhang, Y.; Peng, H.; Zhu, D.; Deng, Z.; Chen, S. L.; Mobli, M.; Jia, X.; Qu, X., Efficient biosynthesis of heterodimeric $\mathrm{C}(3)$-aryl pyrroloindoline alkaloids. Nat. Commun. 2018, 9(1), 4428.

21. Yu, H.; Li, S. M., Two Cytochrome P450 Enzymes from Streptomyces sp. NRRL S-1868 Catalyze Distinct Dimerization of Tryptophan-Containing Cyclodipeptides. Org. Lett. 2019, 21 (17), 7094 7098.

22. Due to the limited availability of NznB protein, we were only able to perform scale up reactions for characterization of $(+)$-naseseazine $B$. Analytical reactions were performed to detect formation of $(+)$-naseseazine $A$ and its structure was assigned by analogy and via comparison of retention time to an analytical standard (Supplementary Figure 11).

23. Gerlt, J. A.; Bouvier, J. T.; Davidson, D. B.; Imker, H. J.; Sadkhin, B.; Slater, D. R.; Whalen, K. L., Enzyme Function InitiativeEnzyme Similarity Tool (EFI-EST): A web tool for generating protein sequence similarity networks. Biochim. Biophys. Acta 2015, 1854 (8), 1019-37.

24. Alkhalaf, L. M.; Barry, S. M.; Rea, D.; Gallo, A.; Griffiths, D.; Lewandowski, J. R.; Fulop, V.; Challis, G. L., Binding of Distinct Substrate Conformations Enables Hydroxylation of Remote Sites in Thaxtomin D by Cytochrome P450 TxtC. J. Am. Chem. Soc. 2019, 141 (1), 216-222.

25. Cryle, M. J.; Bell, S. G.; Schlichting, I., Structural and biochemical characterization of the cytochrome P450 CypX (CYP134A1) from Bacillus subtilis: a cyclo-L-leucyl-L-leucyl dipeptide oxidase. Biochem. 2010, 49(34), 7282-96.

26. Yu, H.; Xie, X.; Li, S. M., Coupling of Guanine with cyclo-lTrp-1-Trp Mediated by a Cytochrome P450 Homologue from Streptomyces purpureus. Org. Lett. 2018, 20 (16), 4921-4925.

27. Shi, J.; Xu, X.; Zhao, E. J.; Zhang, B.; Li, W.; Zhao, Y.; Jiao, R. H.; Tan, R. X.; Ge, H. M., Genome Mining and Enzymatic Total Biosynthesis of Purincyclamide. Org. Lett. 2019.

28. Yu, H.; Xie, X.; Li, S. M., Coupling of cyclo-l-Trp-l-Trp with Hypoxanthine Increases the Structure Diversity of Guanitrypmycins. Org. Lett. 2019, 21 (22), 9104-9108.

29. Liu, J.; Xie, X.; Li, S. M., Guanitrypmycin Biosynthetic Pathways Imply Cytochrome P450 Mediated Regio- and Stereospecific 
Guaninyl-Transfer Reactions. Angew. Chem. Int. Ed. Engl. 2019, 58 (33), 11534-11540.

30. Patteson, J. B.; Cai, W.; Johnson, R. A.; Santa Maria, K. C.; Li, B., Identification of the Biosynthetic Pathway for the Antibiotic Bicyclomycin. Biochem. 2018, 57 (1), 61-65.

31. Meng, S.; Han, W.; Zhao, J.; Jian, X. H.; Pan, H. X.; Tang, G. L., A Six-Oxidase Cascade for Tandem C-H Bond Activation Revealed by Reconstitution of Bicyclomycin Biosynthesis. Angew. Chem. Int. Ed. Engl. $2018,57(3), 719-723$.

32. Vetting, M. W.; Hegde, S. S.; Blanchard, J. S., The structure and mechanism of the Mycobacterium tuberculosis cyclodityrosine synthetase. Nat. Chem. Biol.2010, 6(11), 797-9.

33. Belin, P.; Le Du, M. H.; Fielding, A.; Lequin, O.; Jacquet, M.; Charbonnier, J. B.; Lecoq, A.; Thai, R.; Courcon, M.; Masson, C.; Dugave, C.; Genet, R.; Pernodet, J. L.; Gondry, M., Identification and structural basis of the reaction catalyzed by CYP121, an essential cytochrome P450 in Mycobacterium tuberculosis. Proc. Natl. Acad. Sci. USA 2009, 106 (18), 7426-31.

34. Holm, L., Benchmarking fold detection by DaliLite v.5. Bioinf. 2019, $35(24), 5326-5327$.

35. Lautru, S.; Gondry, M.; Genet, R.; Pernodet, J. L., The albonoursin gene Cluster of $S$ noursei biosynthesis of diketopiperazine metabolites independent of nonribosomal peptide synthetases. Chem. Biol. 2002, $9(12), 1355-64$.

36. Yao, T.; Liu, J.; Liu, Z.; Li, T.; Li, H.; Che, Q.; Zhu, T.; Li, D.; Gu, Q.; Li, W., Genome mining of cyclodipeptide synthases unravels unusual tRNA-dependent diketopiperazine-terpene biosynthetic machinery. Nat. Commun. 2018, 9(1), 4091.

37. Giessen, T. W.; von Tesmar, A. M.; Marahiel, M. A., A tRNAdependent two-enzyme pathway for the generation of singly and doubly methylated ditryptophan 2,5-diketopiperazines. Biochem. 2013, 52 (24), 4274-83.

38. Podust, L. M.; Sherman, D. H., Diversity of P450 enzymes in the biosynthesis of natural products. Nat. Prod. Rep. 2012, 29 (10), 125166.

39. Sherman, D. H.; Li, S.; Yermalitskaya, L. V.; Kim, Y.; Smith, J. A.; Waterman, M. R.; Podust, L. M., The structural basis for substrate anchoring, active site selectivity, and product formation by $\mathrm{P} 450 \mathrm{PikC}$ from Streptomyces venezuelae. J. Biol. Chem. 2006, 281 (36), 26289-97.

40. Carlson, J. C.; Li, S.; Gunatilleke, S. S.; Anzai, Y.; Burr, D. A.; Podust, L. M.; Sherman, D. H., Tirandamycin biosynthesis is mediated by co-dependent oxidative enzymes. Nat. Chem. 2011, 3 (8), 628-33.

41. McIntosh, J. A.; Coelho, P. S.; Farwell, C. C.; Wang, Z. J.; Lewis, J. C.; Brown, T. R.; Arnold, F. H., Enantioselective intramolecular $\mathrm{C}-\mathrm{H}$ amination catalyzed by engineered cytochrome $\mathrm{P} 450$ enzymes in vitro and in vivo. Angew. Chem. Int. Ed. Engl. 2013, 52 (35), 9309-12.

42. Singh, R.; Bordeaux, M.; Fasan, R., P450-catalyzed intramolecular $\mathrm{sp}(3) \mathrm{C}-\mathrm{H}$ amination with arylsulfonyl azide substrates. ACS Catal. 2014, 4(2), 546-552.

43. Baunach, M.; Ding, L.; Bruhn, T.; Bringmann, G.; Hertweck, C., Regiodivergent $\mathrm{N}-\mathrm{C}$ and $\mathrm{N}-\mathrm{N}$ aryl coupling reactions of indoloterpenes and cycloether formation mediated by a single bacterial flavoenzyme. Angew. Chem. Int. Ed. Engl. 2013, 52 (34), 9040-3.

44. Tsutsumi, H.; Katsuyama, Y.; Izumikawa, M.; Takagi, M.; Fujie, M.; Satoh, N.; Shin-Ya, K.; Ohnishi, Y., Unprecedented Cyclization Catalyzed by a Cytochrome P450 in Benzastatin Biosynthesis. J. Am. Chem. Soc. 2018, 140 (21), 6631-6639.

45. Tian, W.; Chen, C.; Lei, X.; Zhao, J.; Liang, J., CASTp 3.0: computed atlas of surface topography of proteins. Nucleic Acids Res. 2018, 46 (W1), W363-W367.

46. Li, X. B.; Li, Y. L.; Zhou, J. C.; Yuan, H. Q.; Wang, X. N.; Lou, H. X., A new diketopiperazine heterodimer from an endophytic fungus Aspergillus niger. J. Asian Nat. Prod. Res. 2015, 17 (2), 182-7.

47. Ding, G.; Jiang, L.; Guo, L.; Chen, X.; Zhang, H.; Che, Y., Pestalazines and pestalamides, bioactive metabolites from the plant pathogenic fungus Pestalotiopsis theae. J. Nat. Prod.2008, 71 (11), 18615.
48. Varoglu, M.; Corbett, T. H.; Valeriote, F. A.; Crews, P., Asperazine, a Selective Cytotoxic Alkaloid from a Sponge-Derived Culture of Aspergillus niger. J. Org. Chem. 1997, 62 (21), 7078-7079.

49. Noble, M. A.; Miles, C. S.; Chapman, S. K.; Lysek, D. A.; MacKay, A. C.; Reid, G. A.; Hanzlik, R. P.; Munro, A. W., Roles of key active-site residues in flavocytochrome P450 BM3. Biochem. J. 1999, 339 (Pt 2), 371-9.

50. Tripathi, S.; Li, H.; Poulos, T. L., Structural basis for effector control and redox partner recognition in cytochrome P450. Science 2013, 340 (6137), 1227-30.

51. Makino, M.; Sugimoto, H.; Shiro, Y.; Asamizu, S.; Onaka, H.; Nagano, S., Crystal structures and catalytic mechanism of cytochrome P450 StaP that produces the indolocarbazole skeleton. Proc. Natl. Acad. Sci. USA 2007, 104 (28), 11591-6.

52. Zhao, B.; Guengerich, F. P.; Bellamine, A.; Lamb, D. C.; Izumikawa, M.; Lei, L.; Podust, L. M.; Sundaramoorthy, M.; Kalaitzis, J. A.; Reddy, L. M.; Kelly, S. L.; Moore, B. S.; Stec, D.; Voehler, M.; Falck, J. R.; Shimada, T.; Waterman, M. R., Binding of two flaviolin substrate molecules, oxidative coupling, and crystal structure of Streptomyces coelicolor A3(2) cytochrome P450 158A2. J. Biol. Chem. 2005, 280 (12), 11599-607.

53. Zhao, B.; Guengerich, F. P.; Voehler, M.; Waterman, M. R., Role of active site water molecules and substrate hydroxyl groups in oxygen activation by cytochrome P450 158A2: a new mechanism of proton transfer. J. Biol. Chem. 2005, 280 (51), 42188-97.

54. He, F.; Mori, T.; Morita, I.; Nakamura, H.; Alblova, M.; Hoshino, S.; Awakawa, T.; Abe, I., Molecular basis for the P450-catalyzed C-N bond formation in indolactam biosynthesis. Nat. Chem. Biol. 2019, $15(12), 1206-1213$.

55. Dumas, V. G.; Defelipe, L. A.; Petruk, A. A.; Turjanski, A. G.; Marti, M. A., QM/MM study of the C-C coupling reaction mechanism of CYP121, an essential cytochrome p450 of Mycobacterium tuberculosis. Proteins 2014, 82 (6), 1004-21.

56. Wang, Y.; Chen, H.; Makino, M.; Shiro, Y.; Nagano, S.; Asamizu, S.; Onaka, H.; Shaik, S., Theoretical and experimental studies of the conversion of chromopyrrolic acid to an antitumor derivative by cytochrome P450 StaP: the catalytic role of water molecules. J. Am. Chem. Soc. 2009, 131 (19), 6748-62.

57. Gebler, J. C.; Woodside, A. B.; Poulter, C. D., Dimethylallyltryptophan Synthase - an Enzyme-Catalyzed Electrophilic Aromatic-Substitution. J. Am. Chem. Soc. 1992, 114 (19), 7354-7360. 


\section{"Structure and Function of NzeB, a Versatile $\mathbf{C}-\mathbf{C}$ and $\mathbf{C}-\mathbf{N}$ Bond Forming Diketopiperazine Dimerase"}

Vikram V. Shende, ${ }^{-}$Yogan Khatri, ${ }^{\dagger}$ Sean A. Newmister, ${ }^{\dagger}$ Jacob N. Sanders, ${ }^{\ominus}$ Petra Lindovska, ${ }^{\Delta}$ Fengan Yu,,$^{-}$Tyler J. Doyon, ${ }^{+}$Justin Kim, ${ }^{\Delta}$ K. N. Houk, ${ }^{\theta, *}$ Mohammad Movassaghi, ${ }^{\Delta, *}$ and David H. Sherman ${ }^{, *_{,}, * *}$

${ }^{\dagger}$ Life Sciences Institute, ${ }^{\sharp}$ Department of Medicinal Chemistry, Program in Chemical Biology, Department of Chemistry, and Department of Microbiology \& Immunology, University of Michigan, Ann Arbor, Michigan, 48109, United States

${ }^{\triangle}$ Department of Chemistry, Massachusetts Institute of Technology, Cambridge, Massachusetts, 02139, United States

${ }^{\theta}$ Department of Chemistry, University of California Los Angeles, Los Angeles, California, 90095, United States

Supporting Information

Table of Contents:

Instrumentation S3

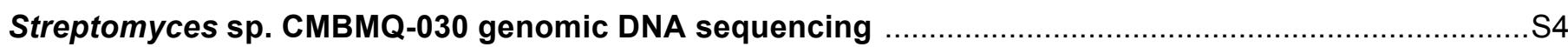

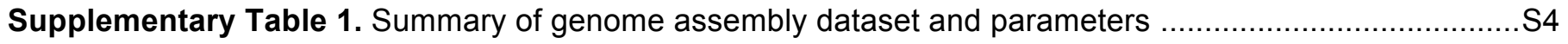

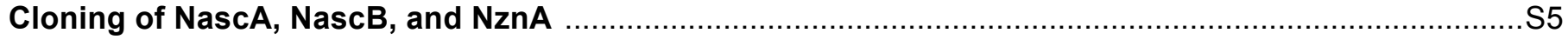

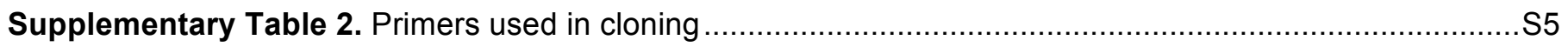

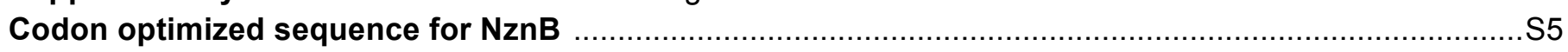

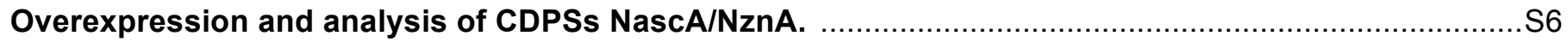

Supplementary Figure 1. HPLC traces for NascA/NznA Expression Extractions ...................................... 6

Supplementary Figure 2. HPLC/MS Anaylsis of Diketopiperazine 6 Isolated From CDPS Culture ...............S7

Supplementary Figure 3. ${ }^{1} \mathrm{H}$ NMR characterization of purified DKP 6 from NascA CDPS overexpression ....S8

Supplementary Figure 4. ${ }^{13} \mathrm{C}$ NMR characterization of purified DKP 6 from NascA CDPS overexpression ..S9

Supplementary Figure 5. HPLC/MS Anaylsis of Diketopiperazine SI-01 Isolated From CDPS Culture ........S10

Supplementary Figure 6. 1H NMR characterization of purified DKP SI-01 from NznA expression ..............S11

Supplementary Figure 7. 13C NMR characterization of purified DKP SI-01 from NznA expression .............S12

Supplementary Figure 8. HPLC Trace for NzeB CDPS Overexpression ............................................. 13

General procedure for overexpression, purification and spectral characterization of cytochrome P450s ........S14

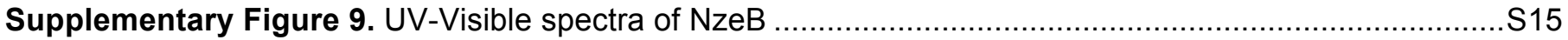

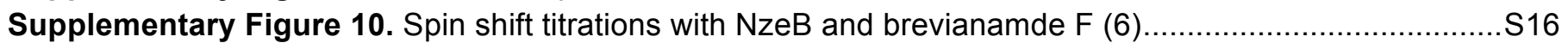

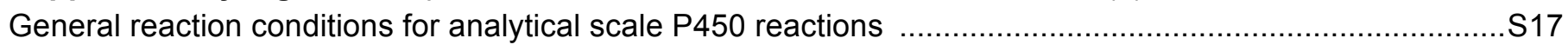

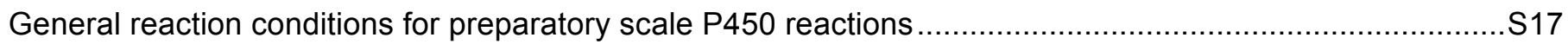

Supplementary Figure 11. HPLC/MS traces with extraction ion chromatograms (EIC) for analytical scale heterodimerization of SI-01 and DKP 6 to form (-)-naseseazine A catalyzed by NznB............................... 19

Supplementary Figure 12. HPLC traces from NzeB catalyzed heterodimerizations ................................... 20

Supplementary Figure 13. HPLC traces from NzeB alanine scanning mutants .....................................S21

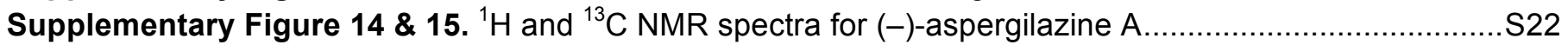

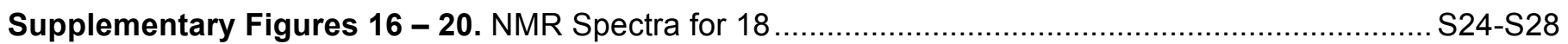

Supplementary Table 3. Cytochromes P450 used to generate sequence similarity network (SSN) .............S29

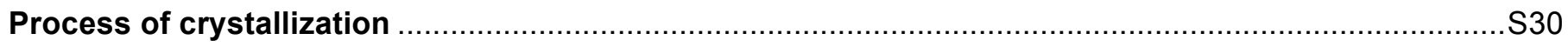

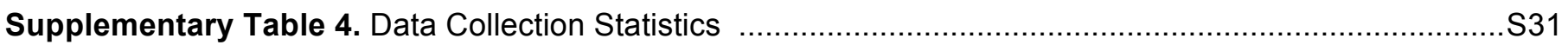

Supplementary Figure 21. Comparison of A) NzeB substrate bound, and B) substrate free active sites......S32

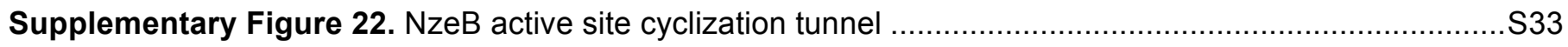

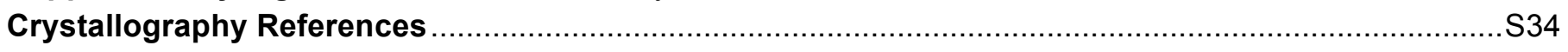


Computational Methods

Supplementary Table 5. Optimized Quantum Mechanical Energies and Geometries

Supplementary Figure 23. Reaction coordinate diagrams for (+)-naseseazine B

Chemistry: General Procedures, Materials, and Instrumentation

Positional Numbering System.

Diketopiperazine bromide (-)-8

meta-(-)-Nin,Nin'-Dicarboxybenzyl iso-Naseseazine C (10) and para-(-)-Nin,Nin'-Dicarboxybenzyl Naseseazine $C(9)$

$(-)$-naseseazine $C(4)$

Supplementary Table 6. Comparison of our ${ }^{13} \mathrm{C}$ NMR data for (-)-naseseazine C (4) and (-)-iso-naseseazine $\mathrm{C}$ (11) with literature data $\left(\mathrm{CD}_{3} \mathrm{OD}\right)$

Supplementary Table 7. Comparison of our 1H NMR data for (-)-naseseazine C (4) and (-)-iso- naseseazine C (11) with literature data (DMSO-d6) 


\section{Instrumentation}

All UV-Visible spectra were acquired using a single beam Molecular Devices Spectra Max M5 spectrophotometer, with a $1 \mathrm{~cm}$ quartz cuvette. Analytical HPLC data was acquired using a Shimadzu HPLC

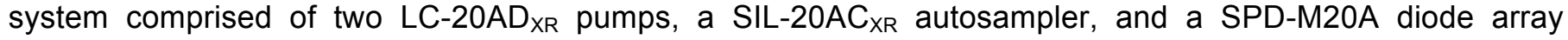
detector. Preparatory HPLC was performed using a Beckman Coulter stack comprised of a System Gold 125 solvent module, 168 detector, and SC100 fraction collector. ${ }^{1} \mathrm{H}$ NMR spectra were recorded on Varian 600 $\mathrm{MHz}$ spectrometers and are reported relative to residual solvent peak $\left(\mathrm{CD}_{2} \mathrm{HOD}: \delta 3.31\right) .{ }^{13} \mathrm{C}$ NMR spectra were reported relative to residual solvent peaks (CD ${ }_{2} \mathrm{HOD}$ : $\delta$ 49.0). 


\section{Streptomyces sp. CMBMQ-030 genomic DNA sequencing}

The genomic DNA of Streptomyces sp. CMB-MQ030 was extracted and purified as prescribed using the Promega Wizard® Genomic DNA Purification Kit. The genomic DNA (2x250 bp paired-end) was sequenced using Illumina HiSeq 2500 sequencing system at University of Michigan DNA Sequencing Core. Briefly, the genomic DNA sample was first sheared to approximately 500 nucleotide average fragment size, then Illuminacompatible sequencing libraries were prepared from those fragments on an Apollo 324 robotic workstation (WaferGen Biosystems), using the Kapa HTP Library Preparation Kit (KAPABiosystem) according to the manufacturer's protocols. Subsequent libraries were sequenced on an Illumina HiSeq 2000, obtaining pairedend sequence data with 100 nucleotide reads at each end, as per recommended protocols from Illumina, Inc. The generated next-generation sequencing data were then de novo assembled using Velvet 1.2.10.

Supplementary Table 1. Summary of genome assembly dataset and parameters

\begin{tabular}{|l|l|l|l|l|l|}
\hline Genome & $\begin{array}{l}\text { Number of } \\
\text { reads }\end{array}$ & k-mer & $\begin{array}{l}\text { Coverage } \\
\text { cutoff }\end{array}$ & N50 & Number of contigs \\
\hline $\begin{array}{l}\text { Streptomyces sp. } \\
\text { (CMB-MQ030) }\end{array}$ & $33,263,004$ & 51 & 10 & 66,868 & 591 \\
\hline
\end{tabular}




\section{Cloning of NascA, NascB, and NznA}

The PCR primers were designed to introduce an Ndel restriction site at the $5^{\prime}$ end of the fragment and a HindlII restriction site $3^{\prime}$ end, respectively. The coding sequences were amplified from the genomic DNA of Streptomyces sp. CMB-MQ030 using primers for the associated target gene. The expression constructs were designed in pET28b vector cloning at $\mathrm{Ndel}$ and Hindlll restriction sites for NascA, NznA and NascB. NznB+09 and NzeB were cloned into pET28b using Quickchange mutagenesis. The nucleotide sequences were confirmed by automated sequencing (University of Michigan DNA Sequencing Core).

Supplementary Table 2. Primers used in cloning NascA, NasB, NznA, NznB, and extending codon optimized sequence of NznB

\begin{tabular}{|c|c|c|c|}
\hline Gene & Primer & Rest. Site & $5^{\prime} \rightarrow 3^{\prime}$ \\
\hline NascA & Forward & Ndel & CCAACCcatatgGTGAACACTTCCCTCGCTGCGGTGGCCGGC \\
\hline NascA & Reverse & HindIII & CCAACCaagcttGCGTTCGGCCGCCCGGTCCCGCAGCAGGAT \\
\hline NascB & Forward & Ndel & CCAACCcatatgGTGACCACCACCGCCACGCTGACCTACCCC \\
\hline NascB & Reverse & HindIII & $\begin{array}{l}\text { CCAACCaagctt } \\
\text { CCAGGTGGCGGGAAGCGCCCGCGGACGGCG }\end{array}$ \\
\hline NznA & Forward & Ndel & CCAACCcatatgATGGCCACACACGCCTCCGCACCCGCACCC \\
\hline NznA & Reverse & HindlII & CCAACCaagcttCTGCTGCGTCACGCGGTCCTTGAGGAG \\
\hline NznB (CO) +09 & Forward & & $\begin{array}{l}\text { GTGGATCCGTATACCAAAGAATGCCGTACCGTGACCACCGC } \\
\text { GCCGGTTCCGCT }\end{array}$ \\
\hline NznB (CO) +19 & Forward & & $\begin{array}{l}\text { GTGATTCGTCCGCAGCCGCATCGTAGCCCGGTGGATCCGTA } \\
\text { TACCAAAGAATGCCGTACCGTGACCACCGCGCCGGTTCCGC } \\
\mathrm{T}\end{array}$ \\
\hline NzeB & Forward & & $\begin{array}{l}\text { ctgagaatctctacttccaaggcgctagcGTGACCACCACCACCGCCACG } \\
\text { CTGACCT }\end{array}$ \\
\hline NzeB & Reverse & & $\begin{array}{l}\text { cctttcgggctttgttagcagccggatcTCACCAGGTGGCGGGGATCGCCC } \\
\text { GC }\end{array}$ \\
\hline
\end{tabular}

\section{Codon optimized sequence for NznB}

ACCACCGCGCCGGTTCCGCTGACCTTCCCGTTTCACGATTGGAGCCAAGAGCTGAGCCCGCACCATGAG CGTCTGCGTGAAGCGGATGCGCCGGTGTGCCCGGTGGTTAGCGAGTACACCGGTGACCGTCTGTGGCT GGTTACCCGTTATGCGACCGCGAAGCGTCTGCTGGAAGATCGTCGTTTCAGCAGCACCGCGGCGATGGC GCCGGGTGCGCCGCGTCAGGAGCCGGTGGAACTGCGTGCGCCGGGCACCACCGGTGATGGTGTGAGC GTTCTGCGTGAGGCGGGTCTGCGTACCGTTTTTACCGAAGGTCTGGGTCCGCGTGCGGCGCGTCGTCAC GGTAAATGGCTGCGTGATCGTGCGGACACCCTGCTGCGTGATGTGGCGGAGTGCGAAGGTCCGGTTGAT CTGGCGGCGGACTTTGCGCAGCCGCTGGCGGTGGCGATGACCAGCCGTGTTCTGCTGGGTGAACTGAG CACCGAGGAAGCGGCGCTGCTGCGTGATCGTACCGATCTGGCGCTGCAGTTTTGCGGTGCGACCGCGG AGGAACAACGTGGTGGCCTGATCGATATTCACCGTTTCTTTACCGCGCATGCGCGTCGTCTGGCGGATG GTCCGGGTGACCACCTGCTGAAGCGTCTGGCGGAGGCGCCGGCGGAAAACGGTCCGCTGGGTGATGCG GCGCTGAGCGAAATTGCGGCGCTGCTGCTGATTGCGGGTTTCCCGACCAGCAGCGGCTTTCTGTGCGGT GCGCTGATCACCCTGCTGCGTCACCCGGAGGCGGTGGGCCGTCTGCGTCGTGATCCGGAACTGATTCC GGACGCGGTTGAGGAACTGCTGCGTCATACCCCGCTGAGCACCGGTGCGGCGAAACGTATGGCGACCG AGGACGCGGATATCGACGGCGTGCGTATTCGTCGTGGTGAGGTGGCGATGGTTAGCCTGGAAGCGGCG AACCACGATCCGGACGCGTTCGACGATCCGGATAGCTTTCGTCCGGAACGTCAAGGTCCGGGTCACCTG GGTTTTGGCCACGGTCCGAACTTTTGCCCGGGTAACCGTCTGGCGCGTTGCCTGATTGATGCGATGGTG CGTGCGGTTGCGCGTCGTCCGGGTCTGCACCTGACCGTTGGTCCGGAGGAAATCCGTTGGCACGAAGG CCTGTTCTTTCGTCGTCCGAAGGCGATTCCGGCGAGCTGG 


\section{Overexpression and analysis of CDPSs NascA/NznA.}

The expression of CDPS was performed by transforming the plasmid into the competent cell $E$. coli strain C41(DE3) and selected on Luria-Bertani (LB) medium plates containing $50 \mu \mathrm{g} / \mathrm{mL}$ kanamycin. A single colony was grown overnight in $5 \mathrm{~mL}$ LB broth containing the same concentration of the antibiotics. The main culture was prepared by inoculating $1 \%$ of each overnight culture into a $2 \mathrm{~L}$ baffled flask containing $1 \mathrm{~L}$ of LB containing $50 \mathrm{\mu g} / \mathrm{mL}$ kanamycin. The cultures were incubated at $37^{\circ} \mathrm{C}$ and $250 \mathrm{rpm}$ for $3-4 \mathrm{~h}$ until the absorbance $A_{590 \mathrm{~nm}}=0.6$. The expression of CDPSs was induced by the addition of $1 \mathrm{mM}$ isopropyl $\beta-D-1-$ thiogalactopyranoside (IPTG). The cultures were incubated further at $18^{\circ} \mathrm{C}$ for $18 \mathrm{~h}$ shaking at $180 \mathrm{rpm}$. Cells were harvested by centrifugation at $5000 \times \mathrm{g}$ at $4^{\circ} \mathrm{C}$ for $15 \mathrm{~min}$ and a $1 \mathrm{~mL}$ aliquot of the supernatant was extracted with $1 \mathrm{~mL} \mathrm{CHCl} 3(3 \mathrm{x})$. The organic extracts were dried and solvents were removed en vacuo. Residue was resuspended in $100 \mathrm{uL}$ of HPLC grade methanol for HPLC analysis in which the samples was resolved using a linear gradient of $5-100 \%$ acetonitrile: water $(0.1 \%$ formic acid) over $30 \mathrm{~min}(1.5 \mathrm{~mL} / \mathrm{min}$ flow rate) on a Phenomenex Luna $5 \mu$ C-18(2) $100 \mathrm{~A}, 250 \times 4.60 \mathrm{~mm} 5$ micron column.

Supplementary Figure 1. HPLC traces for NascA/NznA Expression Extractions

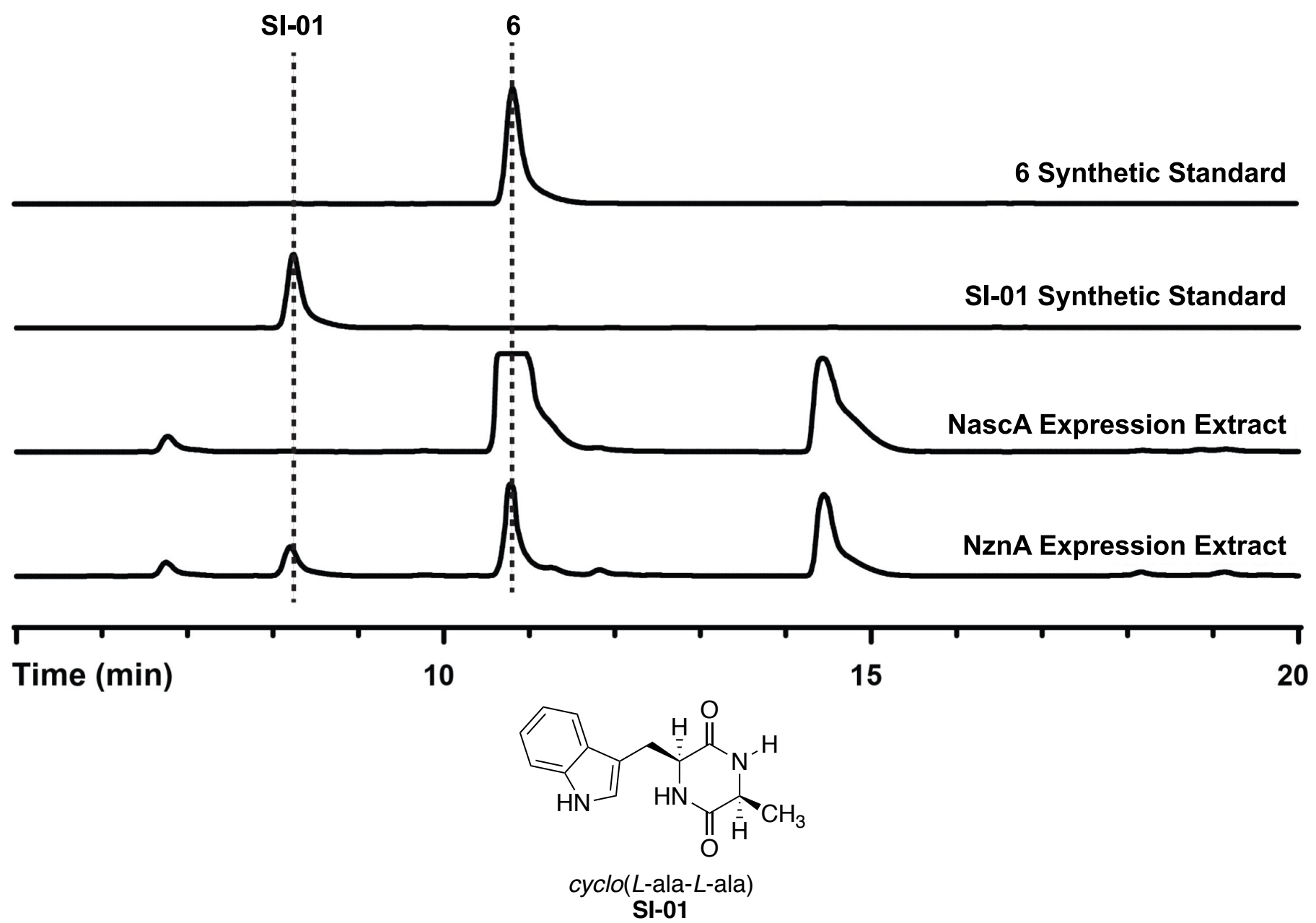


Supplementary Figure 2. HPLC/MS Anaylsis of Diketopiperazine 6 Isolated From CDPS Culture

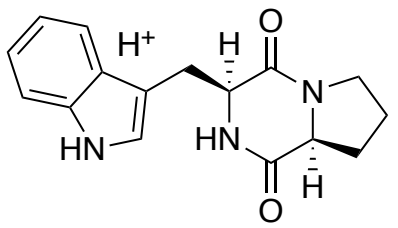

Exact Mass: 284.1394
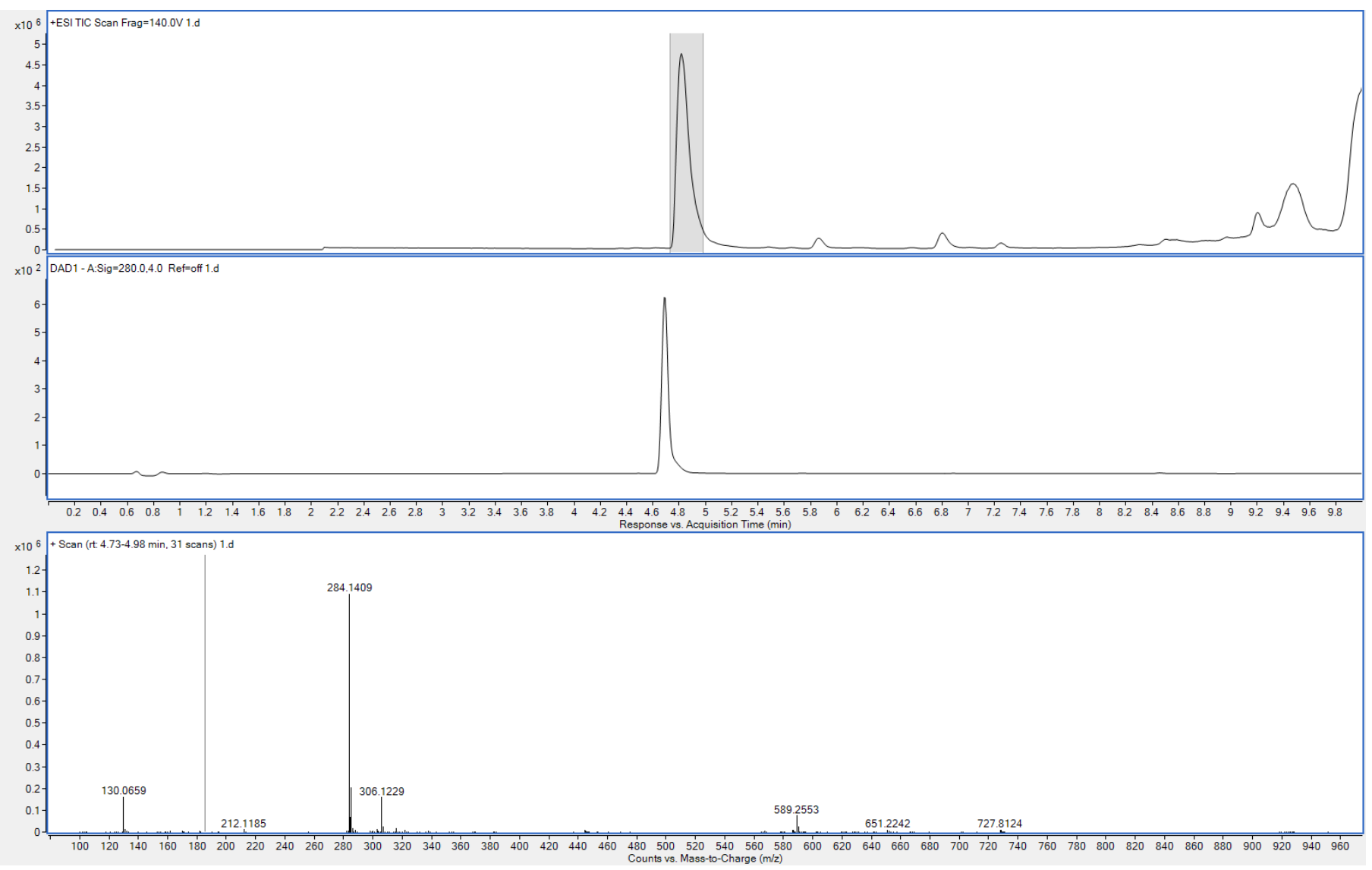
Supplementary Figure 3. ${ }^{1} \mathrm{H}$ NMR characterization of purified DKP 6 from NascA CDPS overexpression

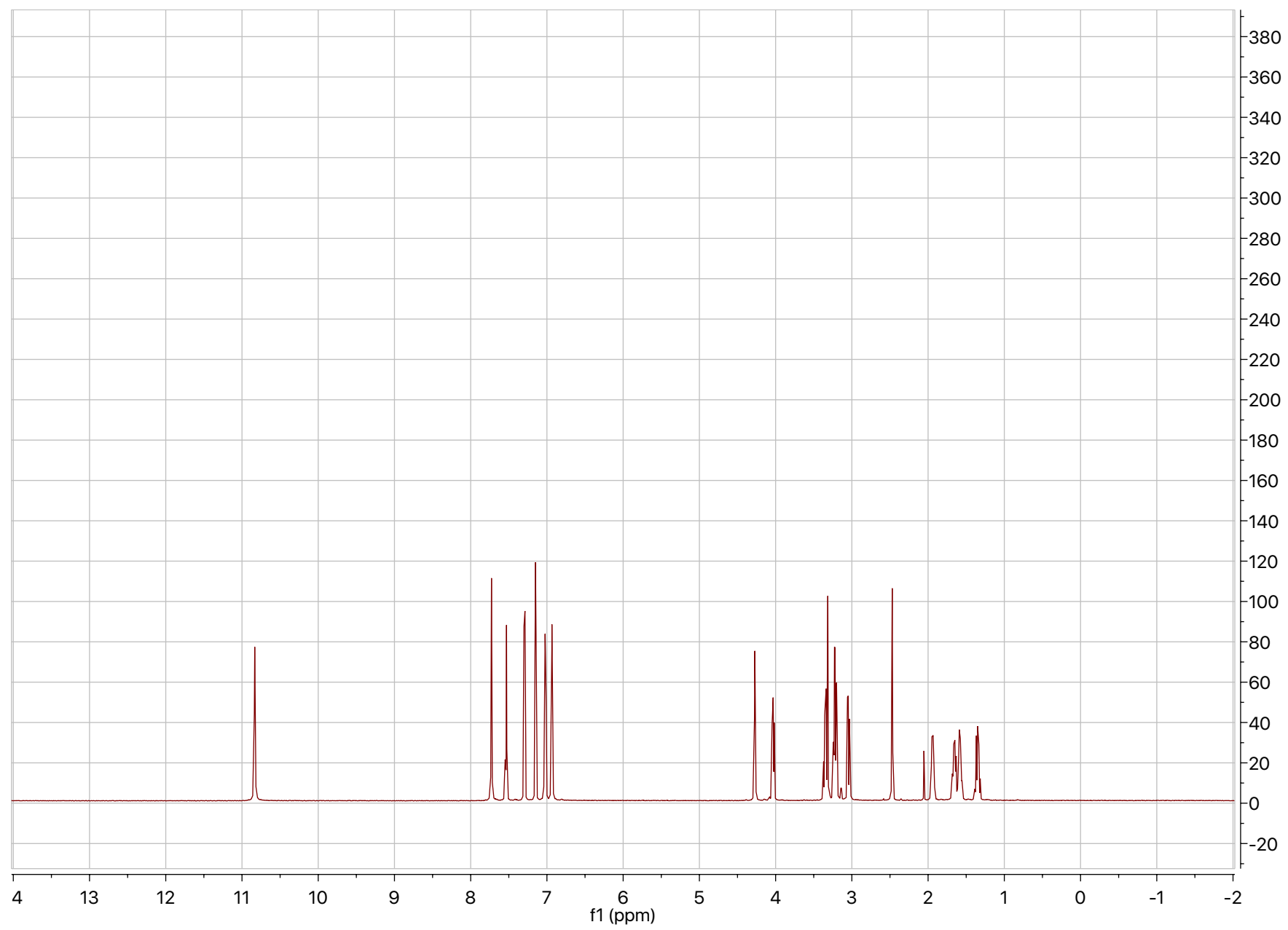

${ }^{1} \mathrm{H}$ NMR (599 MHz, DMSO-d $)_{6} \delta 10.83(\mathrm{~s}, 1 \mathrm{H}), 7.73(\mathrm{~s}, 1 \mathrm{H}), 7.54(\mathrm{~d}, J=7.9 \mathrm{~Hz}, 1 \mathrm{H}), 7.29(\mathrm{~d}, J=8.1 \mathrm{~Hz}, 1 \mathrm{H})$, $7.15(\mathrm{~d}, J=2.2 \mathrm{~Hz}, 1 \mathrm{H}), 7.02(\mathrm{t}, J=7.5 \mathrm{~Hz}, 1 \mathrm{H}), 6.93(\mathrm{t}, J=7.4 \mathrm{~Hz}, 1 \mathrm{H}), 1.94(\mathrm{dtd}, J=9.8,6.9,2.7 \mathrm{~Hz}, 1 \mathrm{H})$, $1.66(\mathrm{ddd}, J=19.1,9.1,5.6 \mathrm{~Hz}, 1 \mathrm{H}), 1.59(\mathrm{ddp}, J=12.2,8.4,4.6,4.1 \mathrm{~Hz}, 1 \mathrm{H}), 1.35(\mathrm{dtd}, J=12.1,10.3,7.8$ $\mathrm{Hz}, 1 \mathrm{H})$. 
Supplementary Figure 4. ${ }^{13} \mathrm{C}$ NMR characterization of purified DKP 6 from NascA CDPS overexpression

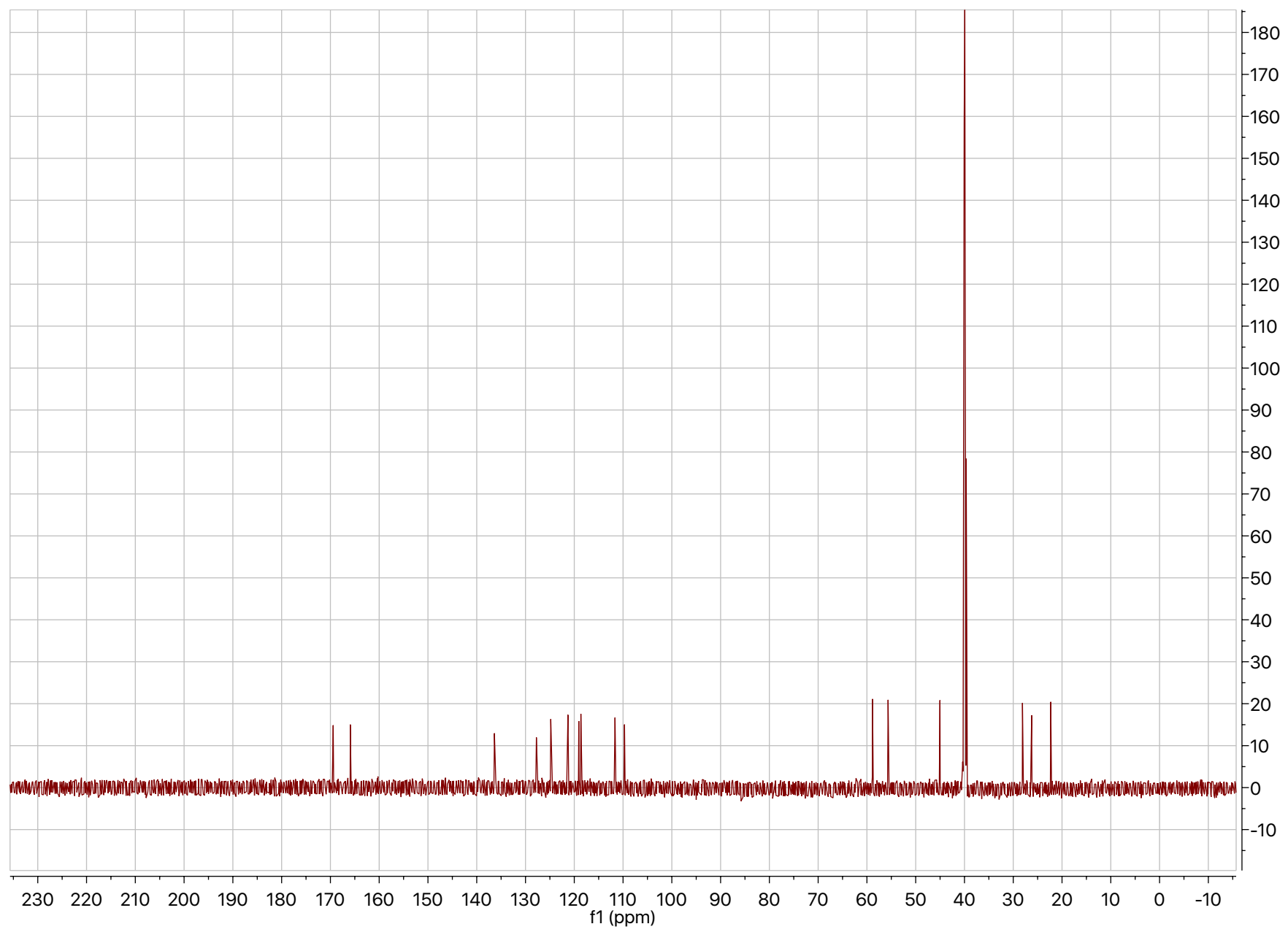

${ }^{13} \mathrm{C}$ NMR (151 MHz, dmso) $\delta 169.43,165.92,136.37,127.77,124.82,121.30,119.08,118.65,111.65,109.70$, $58.85,55.66,45.03,28.11,26.23,22.31$. 
Supplementary Figure 5. HPLC/MS Anaylsis of Diketopiperazine SI-01 Isolated From CDPS Culture

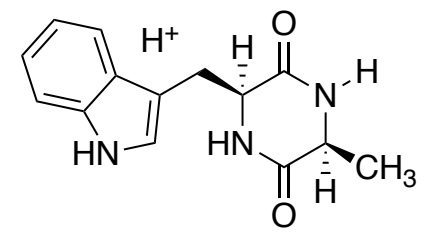

SI-01

Exact Mass: 258.1237
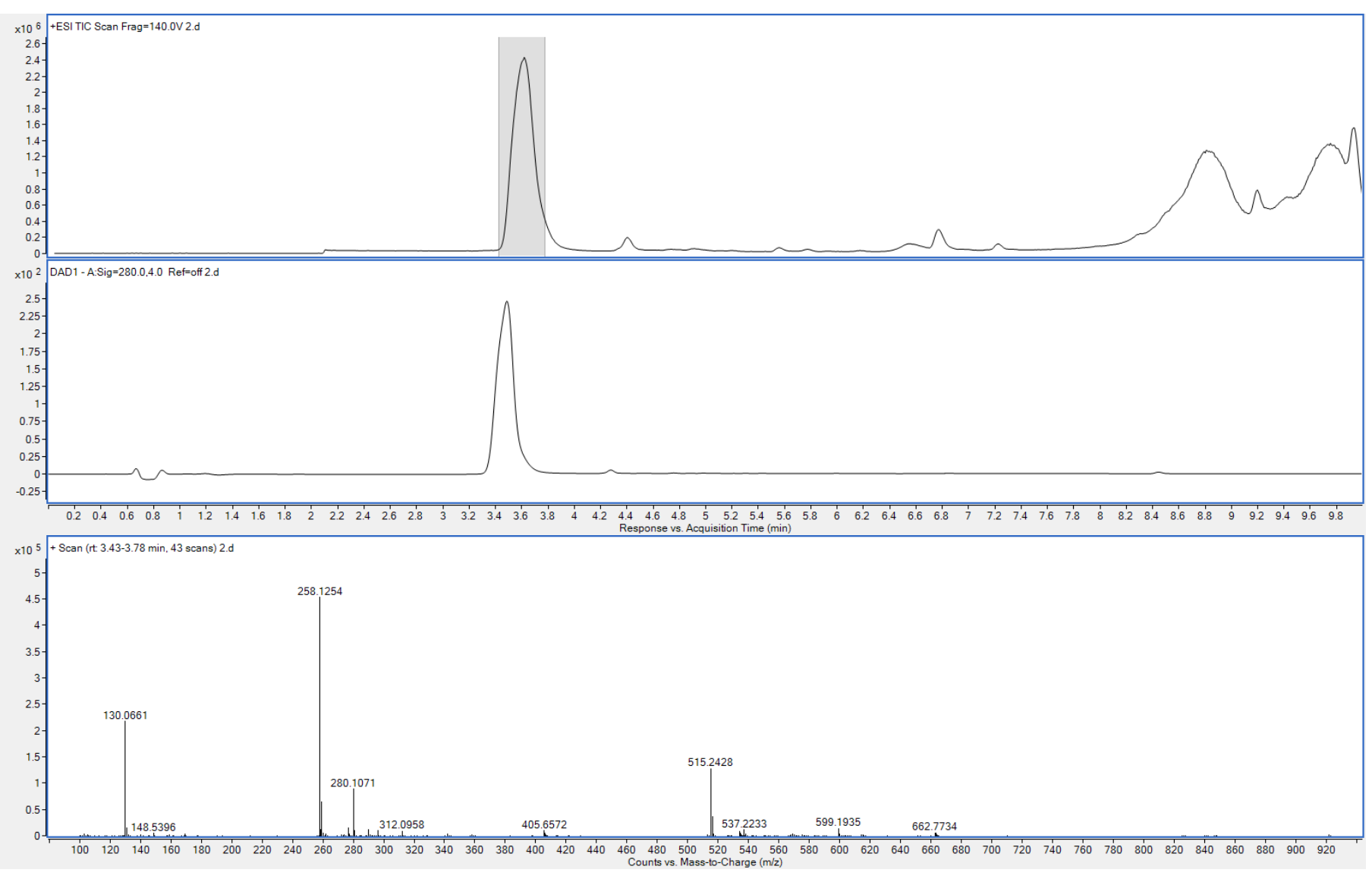
Supplementary Figure 6. ${ }^{1} \mathrm{H}$ NMR characterization of purified DKP SI-01 from NznA expression.

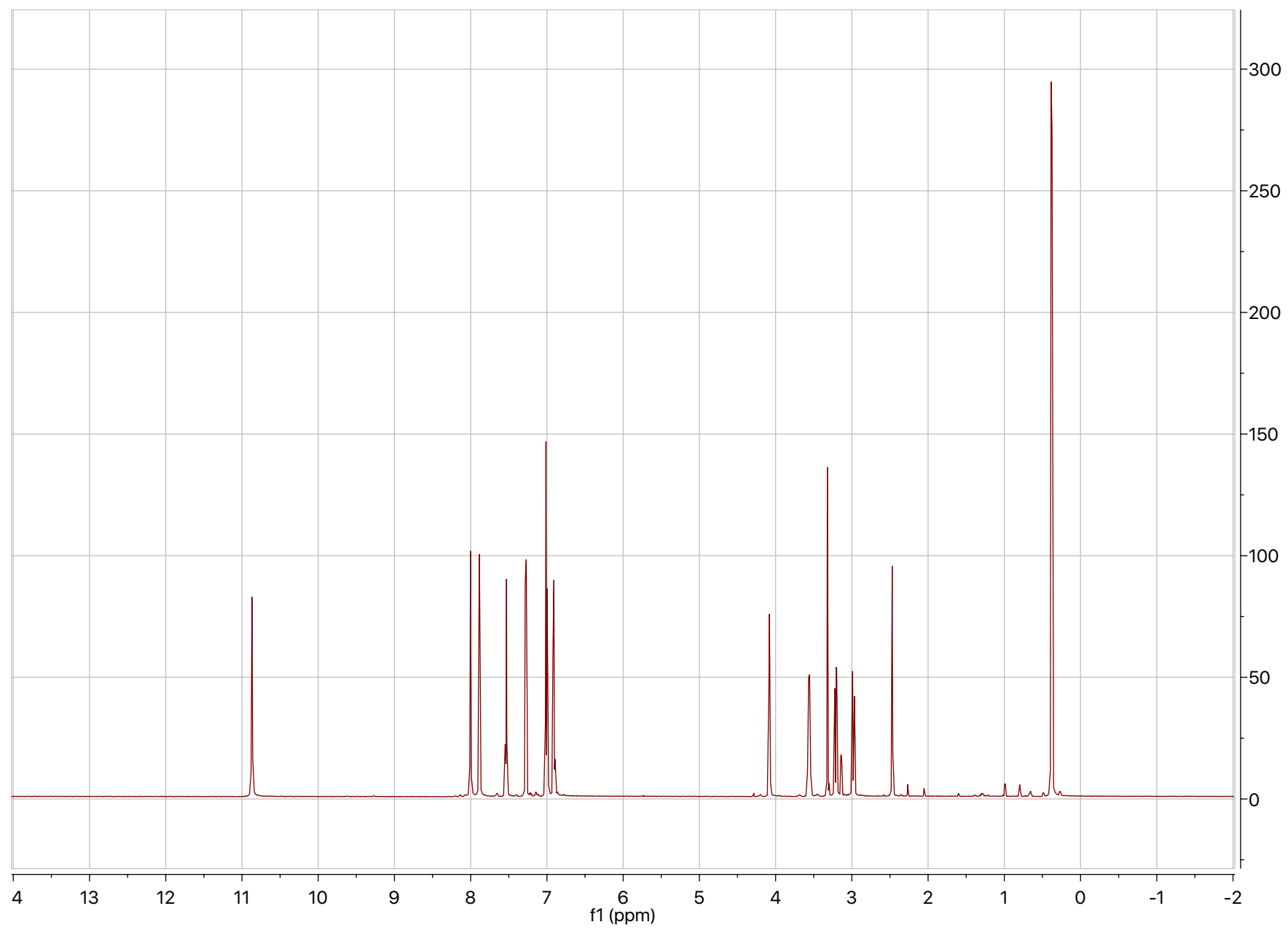

${ }^{1} \mathrm{H}$ NMR $\left.(599 \mathrm{MHz} \text {, DMSO-d })_{6}\right) \delta 10.87(\mathrm{~s}, 1 \mathrm{H}), 8.00(\mathrm{~s}, 1 \mathrm{H}), 7.89(\mathrm{~s}, 1 \mathrm{H}), 7.54(\mathrm{~d}, J=7.9 \mathrm{~Hz}, 1 \mathrm{H}), 7.28(\mathrm{~d}, J=$ $8.0 \mathrm{~Hz}, 1 \mathrm{H}), 7.04-6.97(\mathrm{~m}, 2 \mathrm{H}), 6.91(\mathrm{t}, J=7.4 \mathrm{~Hz}, 1 \mathrm{H}), 4.08(\mathrm{~d}, J=2.7 \mathrm{~Hz}, 1 \mathrm{H}), 3.56(\mathrm{dd}, J=7.0,2.0 \mathrm{~Hz}$, $1 \mathrm{H}), 3.32(\mathrm{~s}, 1 \mathrm{H}), 3.21(\mathrm{dd}, J=14.4,4.1 \mathrm{~Hz}, 1 \mathrm{H}), 2.98(\mathrm{dd}, J=14.4,4.6 \mathrm{~Hz}, 1 \mathrm{H}), 2.47(\mathrm{t}, J=1.9 \mathrm{~Hz}, 1 \mathrm{H}), 0.38$ $(\mathrm{d}, J=7.0 \mathrm{~Hz}, 3 \mathrm{H})$. 
Supplementary Figure 7. ${ }^{13} \mathrm{C}$ NMR characterization of purified DKP SI-01 from NznA expression.

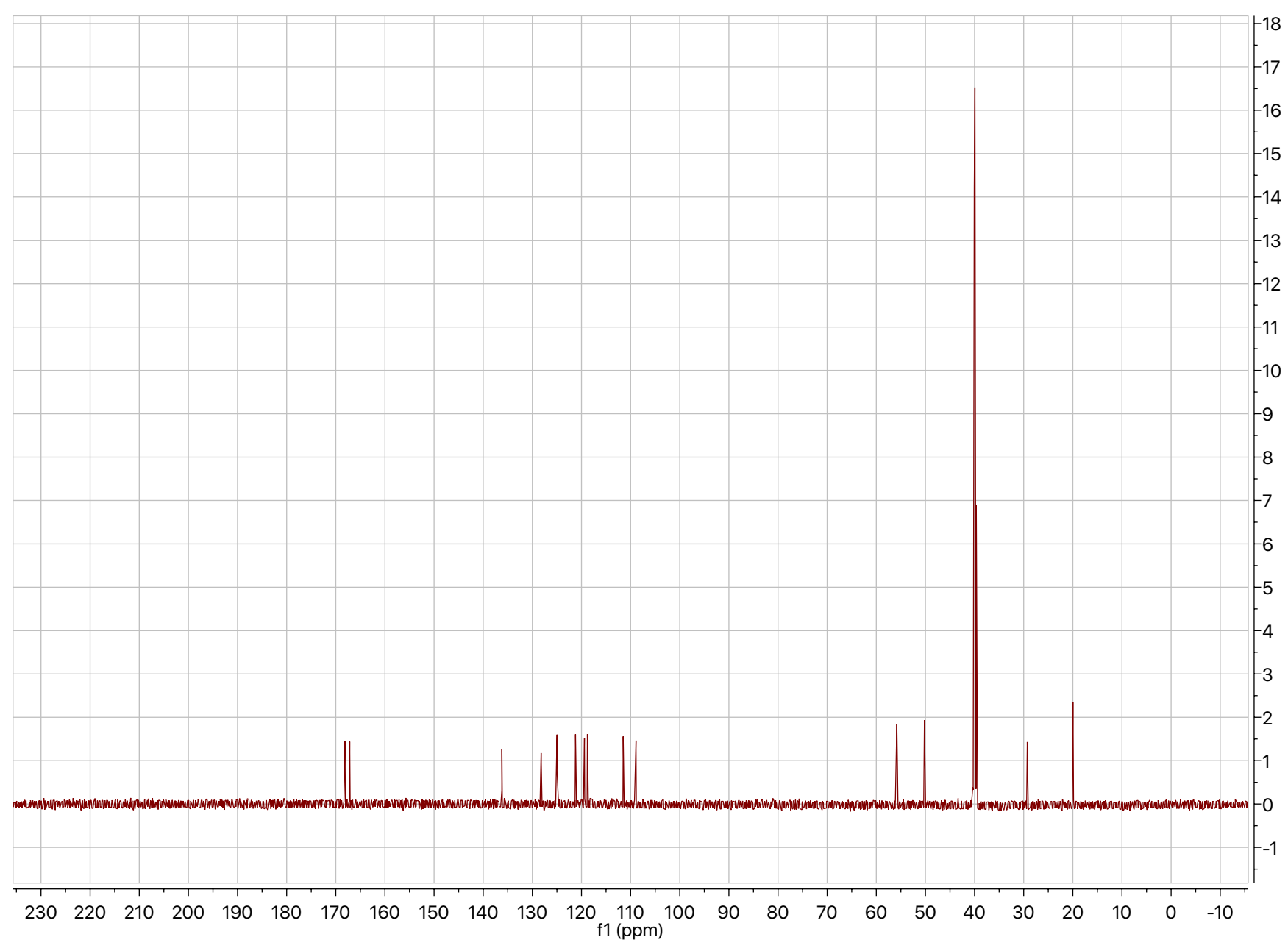

${ }^{13} \mathrm{C}$ NMR (151 MHz, dmso) $\delta 168.14,167.16,136.20,128.22,124.98,121.22,119.40,118.80,111.51,108.91$, $55.82,50.20,29.27,19.98$. 
Supplementary Figure 8. HPLC trace for NzeB CDPS overexpression

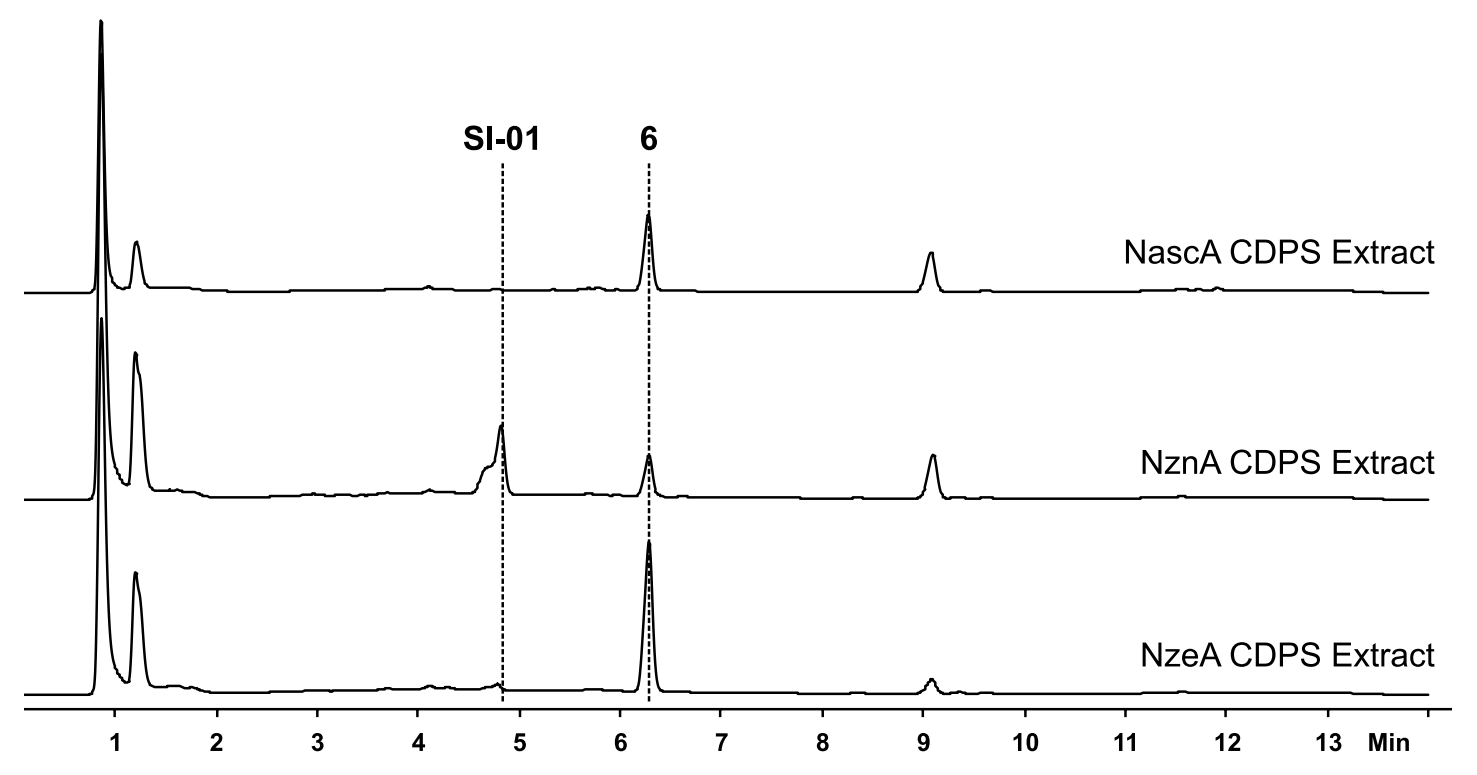


General procedure for overexpression, purification and spectral characterization of cytochrome P450s The expression of $\mathrm{P} 450$ s was performed by transforming the plasmid into the competent cell $E$. coli strain C41(DE3) and selected on Luria-Bertani (LB) medium plates containing $50 \mu \mathrm{g} / \mathrm{mL}$ kanamycin. A single colony was grown overnight in LB broth containing the same concentration of the antibiotics. The main culture was prepared by inoculating $1 \%$ of each overnight culture into a $2 \mathrm{~L}$ baffled flask containing $500 \mathrm{ml}$ of Terrific Broth (TB) containing $50 \mu \mathrm{g} / \mathrm{mL}$ kanamycin. The cultures were incubated at $37^{\circ} \mathrm{C}$ and $250 \mathrm{rpm}$ for $3-4 \mathrm{~h}$ unless the absorbance $A_{590 \mathrm{~nm}}=1$. The expression was induced by the addition of $0.8 \mathrm{mM}$ isopropyl $\beta-D-1$ thiogalactopyranoside (IPTG) and $1.0 \mathrm{mM}$ delta-5-aminolevulinic acid (w/v). The cultures were incubated further for $36 \mathrm{~h}, 28^{\circ} \mathrm{C}$ at $160 \mathrm{rpm}$. Cells were harvested by centrifugation at $5000 \times \mathrm{g}$ at $4^{\circ} \mathrm{C}$ for $10 \mathrm{~min}$ and the cell pellet was stored at $-80^{\circ} \mathrm{C}$ until purification of the protein.

The cell pellet was resuspended in $5 \%$ culture volume of lysis buffer $(50 \mathrm{mM}$ Tris, $\mathrm{pH} 7.4$ containing $50 \mathrm{mM}$ $\mathrm{NaCl}, 2 \%$ glycerol, $0.5 \mathrm{mM}$ EDTA, $10 \mathrm{mM}$ beta-mercaptoethanol and $1 \mathrm{mM}$ phenylmethane sulfonyl fluoride (PMSF) and disrupted by sonication. The cell lysate was centrifugated (35,000 rpm for $35 \mathrm{~min})$ and filtered (0.2 $\mu \mathrm{M}$ Millipore filter). The soluble $\mathrm{His}_{6}$-tagged $\mathrm{NasB}$ was purified by affinity chromatography using Ni-NTA (Qiagen) column and the collected fractions were analyzed by SDS-PAGE. The suitable red fractions were pooled and dialyzed at $4^{\circ} \mathrm{C}$ three times using $50 \mathrm{mM}$ Tris, pH 7.4 containing $10 \%$ glycerol, $50 \mathrm{mM} \mathrm{NaCl}$ and 1 $\mathrm{mM}$ dithiothreitol (DTT) against a total of $6 \mathrm{~L}$ buffer. 
Supplementary Figure 9. UV-Visible spectra of NzeB

UV-Vis spectra for the purified NzeB was recorded at room temperature in buffer $(50 \mathrm{mM}$ Tris- $\mathrm{HCl}$ buffer, $\mathrm{pH}$ 7.4 containing $5 \%$ glycerol) was used for the spectral measurements of the oxidized and reduced form. NzeB was reduced by the addition of a small amount of sodium dithionite. The concentration of the P450s was estimated by CO-difference spectra, assuming $\varepsilon(459-490)=91 \mathrm{mM}-1 \mathrm{~cm}-1$ according to the method of Omura and Sato .

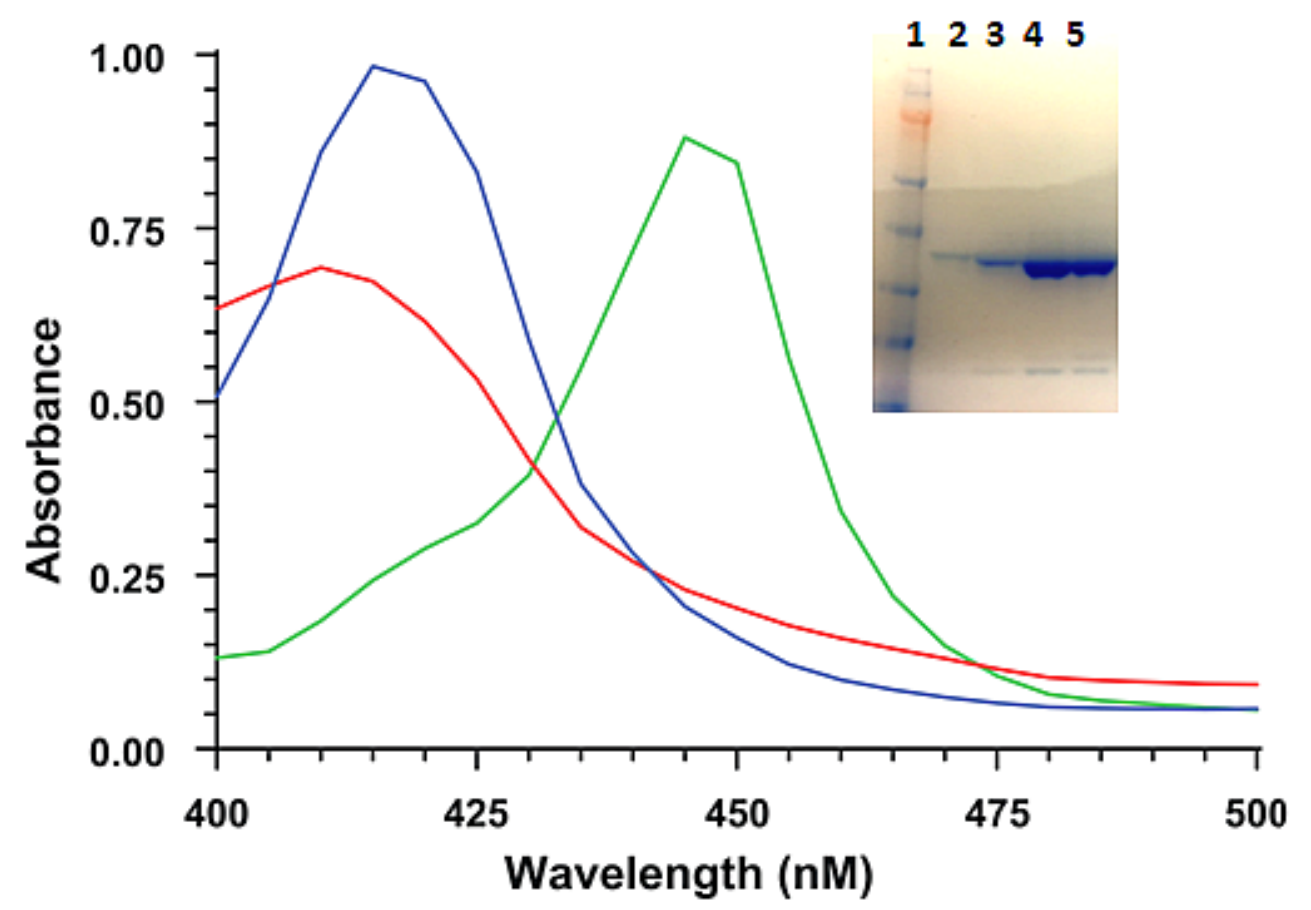

The UV-Vis spectra of oxidized (blue line), sodium dithionite reduced (red line) and CO-bound (green line) of purified NzeB are shown. The inset showed the SDS-PAGE of NzeB purification, Lane 1: marker (SeeBlue Plus2, Novex), 2-5: purified NzeB fractions 
Supplementary Figure 10. Spin shift titrations with NzeB and brevianamde F (6)

The spin-state shift caused by binding of 6 with NzeB was assayed at room temperature under aerobic conditions using an UV-Vis spectrophotometer. The high-spin spectrum of NzeB $(5 \mu \mathrm{M})$ was obtained by adding 10 to $100 \mu \mathrm{M}$ of 6 .

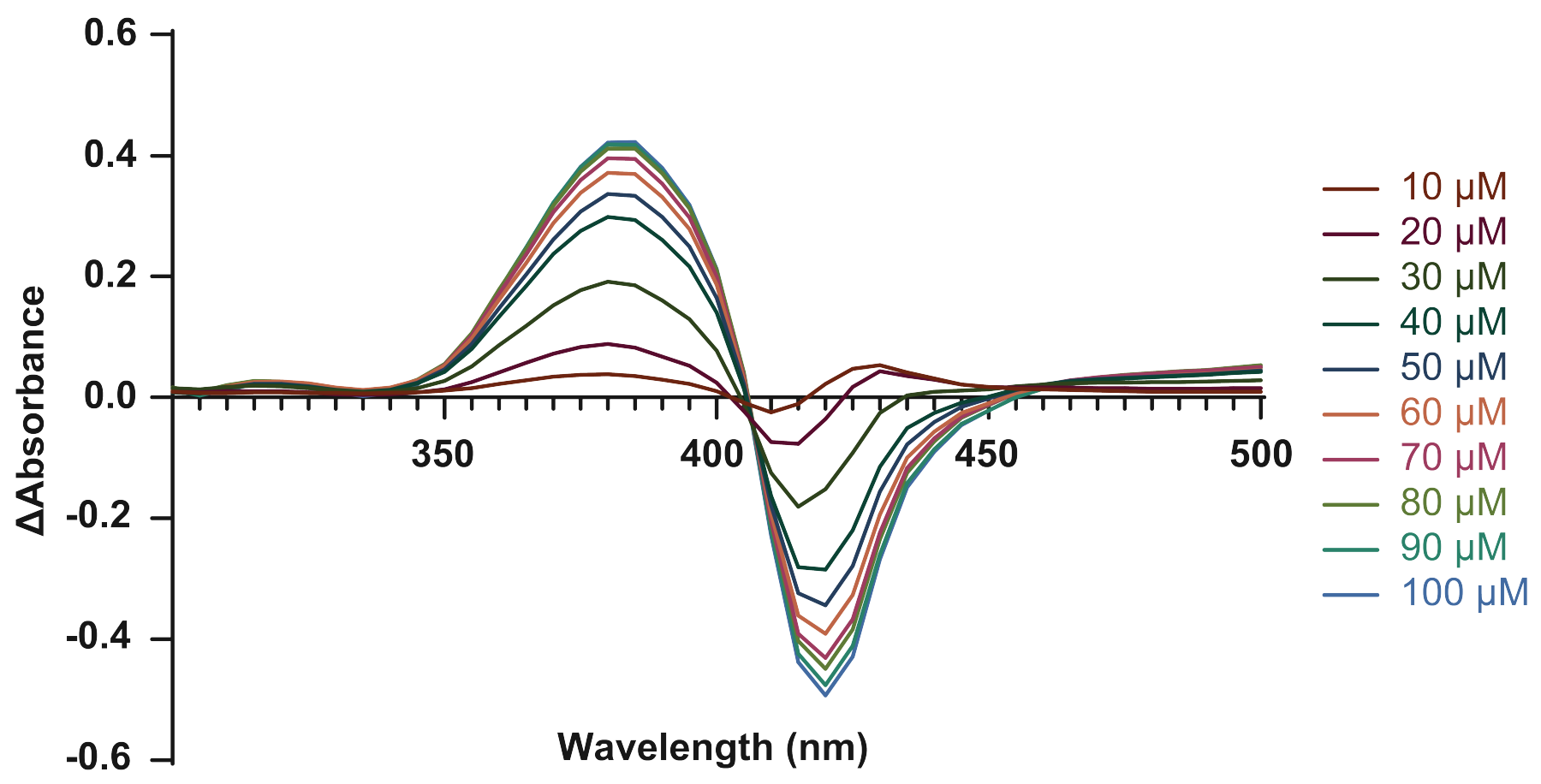

The overlaid difference spectra obtained by subtracting the spectrum for ligand-free NzeB from the successive spectra for substrate-bound accumulated during the titration between 10 and $100 \mu \mathrm{M} 6$ are shown. The Soret band maximum is gradually shifted from $417 \mathrm{~nm}$ to $393 \mathrm{~nm}$ during the addition of increasing concentrations of 6. 


\section{General reaction conditions for analytical scale $\mathbf{P 4 5 0}$ reactions}

The conversion of DKPs by P450s were carried out with the heterologous redox electron partners Fdx and FdR from S. olerecea. A protein ratio of P450: Fdx: FdR of 10: 20: 6 was used. The in vitro reaction mixture included $50 \mathrm{mM}$ Tris- $\mathrm{HCl}, \mathrm{pH} 7.4,10 \mu \mathrm{M} \mathrm{P} 450,20 \mu \mathrm{M} \mathrm{Fdx}$ and $6 \mu \mathrm{M} \mathrm{FdR}$ at the end volume of $250 \mu \mathrm{L}$. $3 \mathrm{mM}$ of DKP dissolved in DMSO was added, followed by 1 unit of glucose-6-phosphate dehydrogenase (from S. cerevisiae and a $500 \mathrm{mM}$ glucose-6-phosphate. The reaction was initiated by adding NADPH (1 mM). Two of the control reactions including all the contents except for NADPH or P450 were used as controls. The reaction was incubated at $30^{\circ} \mathrm{C}$ for $1 \mathrm{~h}$ agitating at $600 \mathrm{rpm}$ in a thermoshaker (Multi-thermoshaker, Benchmark). The conversion was stopped by adding an equal volume of chloroform and was extracted with chloroform for 3 times. The samples were dried under a stream of nitrogen and the residue was resuspended in $100 \mu \mathrm{L}$ of HPLC grade methanol and analyzed by HPLC in which the reaction mixture was resolved using a linear gradient of $5-100 \%$ acetonitrile: water $(0.1 \%$ formic acid) over $30 \mathrm{~min}(1.5 \mathrm{~mL} / \mathrm{min}$ flow rate $)$ on a Phenomenex Luna $5 \mu$ C-18(2) 100A, $250 \times 4.60 \mathrm{~mm} 5$ micron column.

\section{General reaction conditions for preparatory scale $\mathbf{P} 450$ reactions}

Large scale reaction were performed in $10 \mathrm{~mL}$ volumes with reaction conditions as described above. After 2 hours, reactions were quenched with $30 \mathrm{~mL}$ of methanol, transferred to a $50 \mathrm{~mL}$ conical tube and vortexed on the highest setting for 1 minute. This mixture was then passed through a column of Celite $@$ followed by $20 \mathrm{~mL}$ of methanol to was the filter cake. Water and solvents were removed en vacuo, and residue was resuspended in HPLC grade methanol with sonication. Insoluble particulates were removed via filtration through $0.2 \mu \mathrm{m}$ syringe filter and products were directly purified by preparatory-HPLC.

\section{Prep-Scale Synthesis of (-)-naseseazine C (4)}

Procedure above was followed with DKP 6, as a substrate and NascB as P450. The filtered solution was then purified via preparative HPLC (Luna preparative HPLC column, C18, $5 \mu \mathrm{m}, 21.2 \times 250 \mathrm{~mm}$;) using gradient of $5 \%$ to $100 \%$ acetonitrile in water containing $0.1 \%$ formic acid at the flow of $7.0 \mathrm{~mL} / \mathrm{min}$ for 60 min. The retention time $\left(t_{R}\right)$ of $\mathbf{4}$ was $31.2 \mathrm{~min}$. The product fractions were pooled, solvent was removed en vacuo, to give $8 \mathrm{mg}$ of (-)-naseseazine $\mathrm{C}$ which was used for detailed NMR characterization without any further purification.

\section{Prep-Scale Synthesis of (+)-naseseazine B (2)}

Procedure above was followed with DKP 6, as a substrate and NznB+09 as P450. The filtered solution was then purified via preparative HPLC (Luna preparative HPLC column, C18, $5 \mu \mathrm{m}, 21.2 \times 250 \mathrm{~mm}$;) using gradient of $5 \%$ to $100 \%$ acetonitrile in water containing $0.1 \%$ formic acid at the flow of $7.0 \mathrm{~mL} / \mathrm{min}$ for $60 \mathrm{~min}$. The retention time $\left(t_{R}\right)$ of 2 was $34.1 \mathrm{~min}$. The product fractions were pooled, solvent was removed en vacuo, to give $11 \mathrm{mg}$ of (+)-naseseazine $B$ which was used for detailed NMR characterization without any further purification.

\section{Prep-Scale Synthesis of (-)-aspergilazine A (5)}

Procedure above was followed with DKP 6, as a substrate and NzeB as P450. The filtered solution was then purified via preparative HPLC (Luna preparative HPLC column, C18, $5 \mu \mathrm{m}, 21.2 \times 250 \mathrm{~mm}$;) using gradient of $5 \%$ to $100 \%$ acetonitrile in water containing $0.1 \%$ formic acid at the flow of $7.0 \mathrm{~mL} / \mathrm{min}$ for $60 \mathrm{~min}$. The retention time $\left(t_{R}\right)$ of $\mathbf{5}$ was $36.6 \mathrm{~min}$. The product fractions were pooled, solvent was removed en vacuo, to give $2 \mathrm{mg}$ of (+)-naseseazine $\mathrm{B}, 6 \mathrm{mg}$ of (-)-naseseazine $\mathrm{C}$, and $3 \mathrm{mg}$ of (-)-aspergilazine $\mathrm{A}$ which was used for detailed NMR characterization without any further purification.

\section{Prep-Scale Synthesis of 18}


Procedure above was followed with DKPs 6 and 17, as a substrates and NzeB as P450. The filtered solution was then purified via preparative HPLC (Luna preparative HPLC column, C18, $5 \mu \mathrm{m}, 21.2 \times 250 \mathrm{~mm}$;) using gradient of $5 \%$ to $100 \%$ acetonitrile in water containing $0.1 \%$ formic acid at the flow of $7.0 \mathrm{~mL} / \mathrm{min}$ for $60 \mathrm{~min}$. The retention time $\left(t_{R}\right)$ of 18 was $42.1 \mathrm{~min}$. The product fractions were pooled, solvent was removed en vacuo, to give approximately $1 \mathrm{mg}$ of $\mathbf{1 8}$ which was used for detailed NMR characterization without any further purification. 
Supplementary Figure 11. HPLC/MS traces with extraction ion chromatograms (EIC) for analytical scale heterodimerization of SI-01 and DKP 6 to form (-)-naseseazine A catalyzed by NznB.
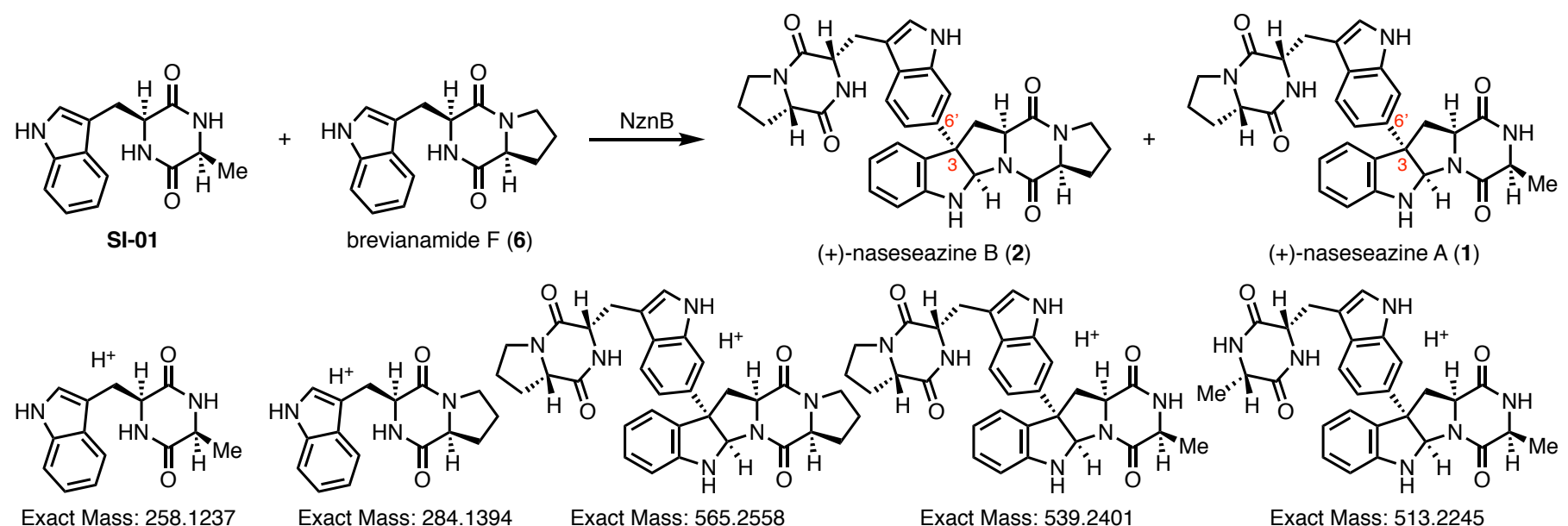

Exact Mass: 258.1237

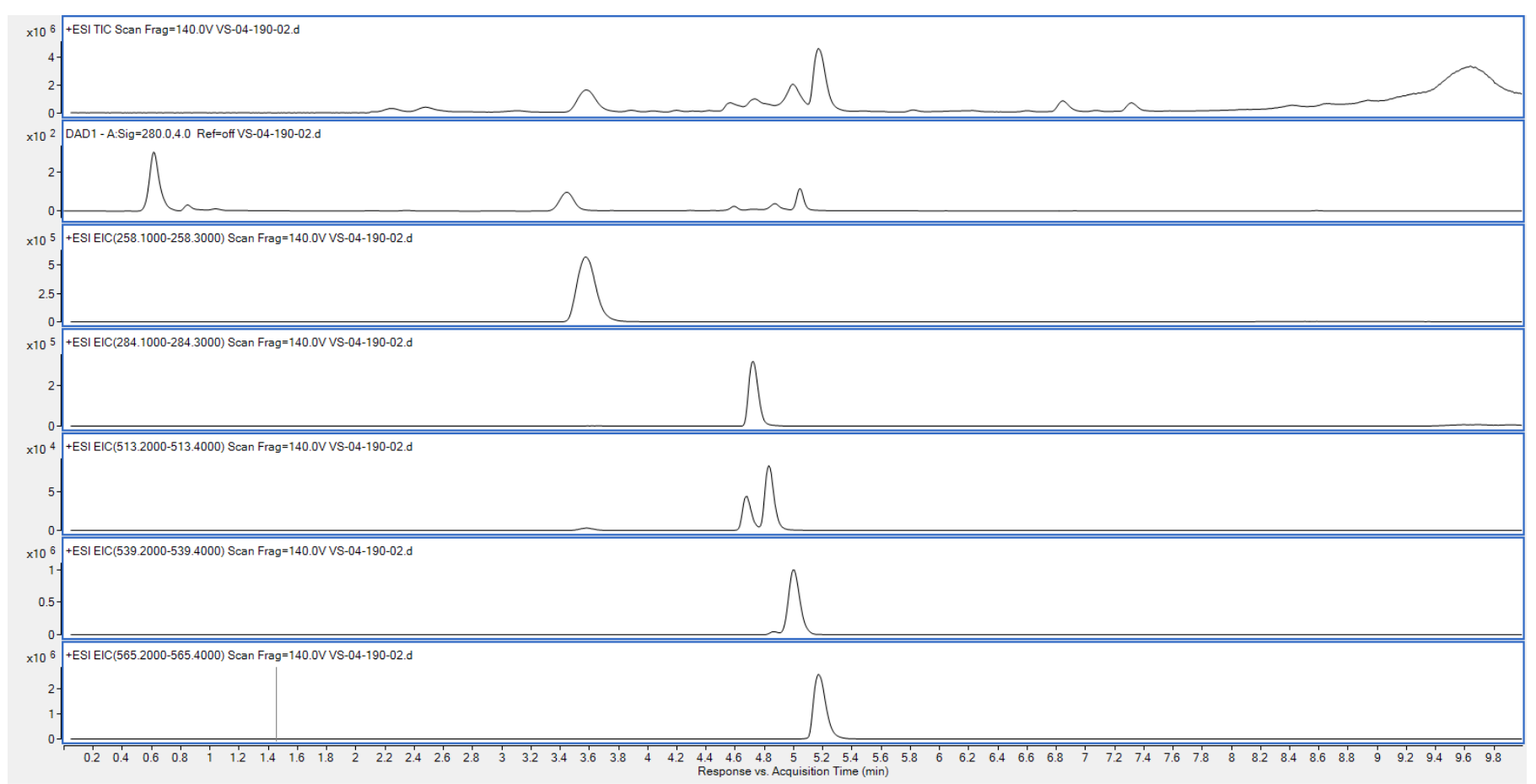


Supplementary Figure 12. HPLC traces from NzeB catalyzed heterodimerizations.
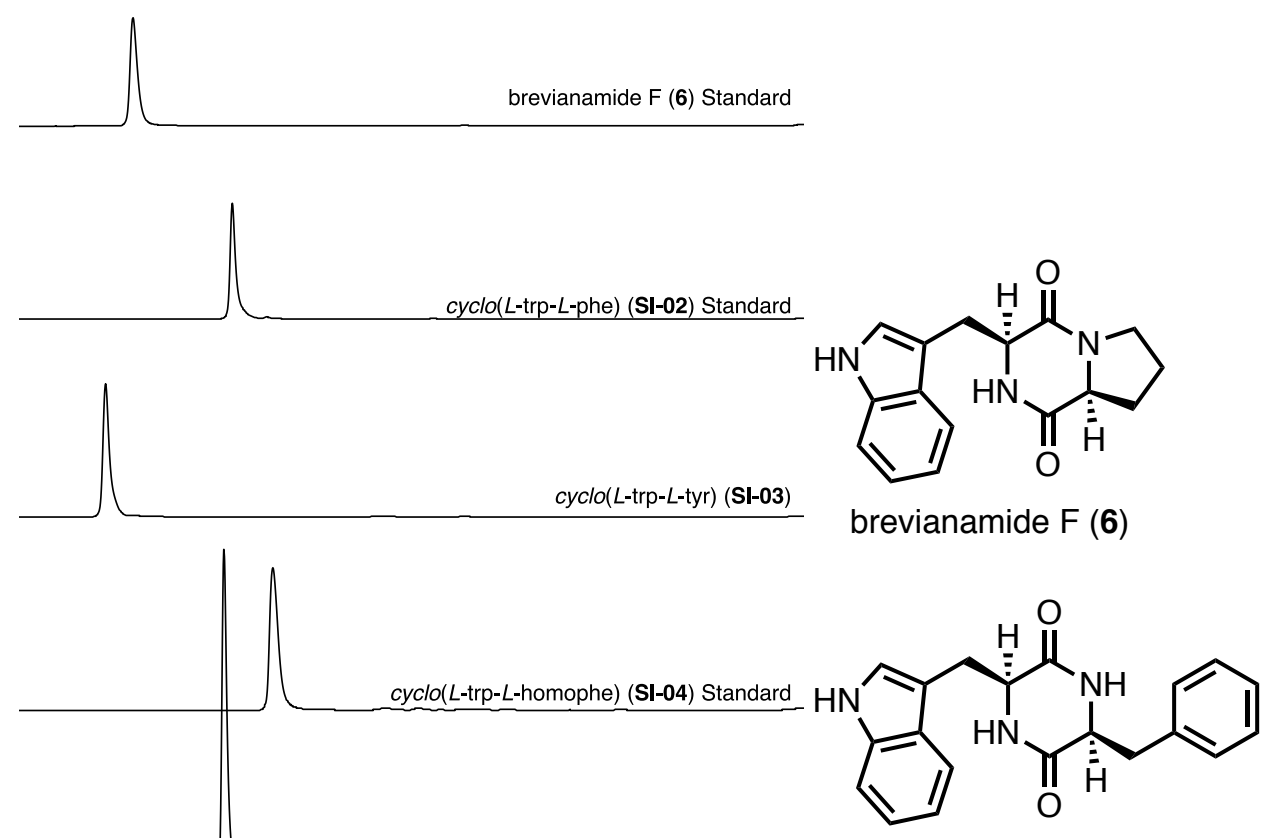

$c y c l o(L-\operatorname{trp}-L$-trp) (17)

cyclo(L-trp-L-phe) (SI-02)

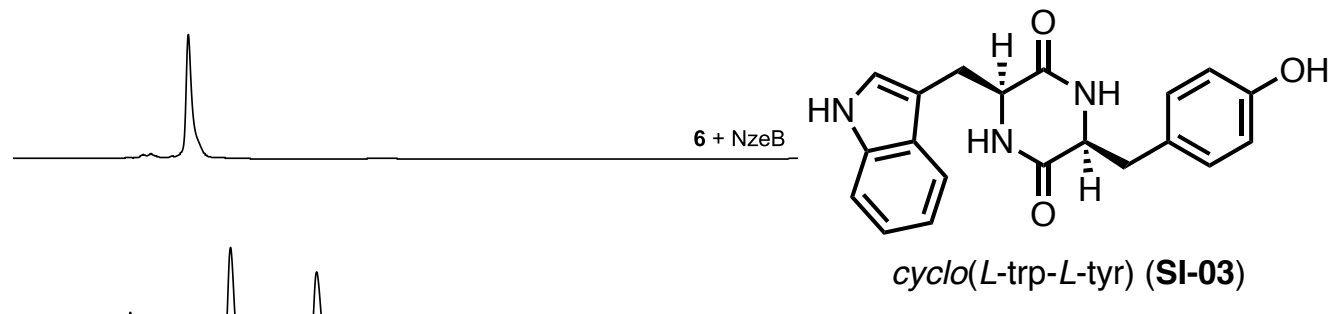

$6+\mathrm{SI}-02+\mathrm{NzeB}$
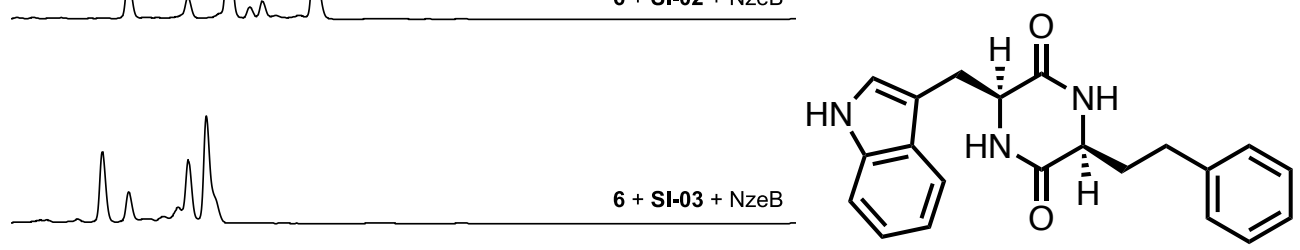

cyclo(L-trp-L-homophe) (SI-04)

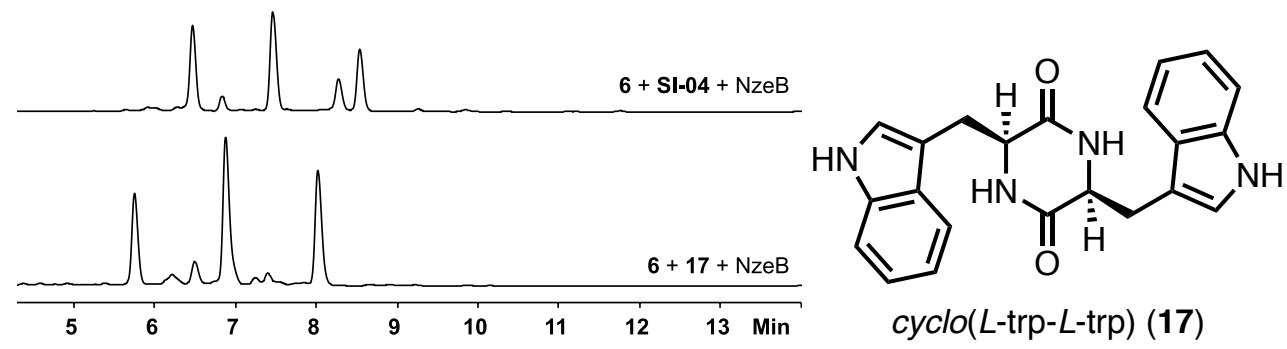

All heterodimerizations of DKP 6 with DKPS SI-02-SI-04 show formation of (-)-naseseazine $C(4)$ with the heterodimerization of 6 with $c y c l o(L-T r p-L-T r p)(17)$ giving the least formation of 4. 
Supplementary Figure 13. HPLC traces from NzeB alanine scanning mutants.

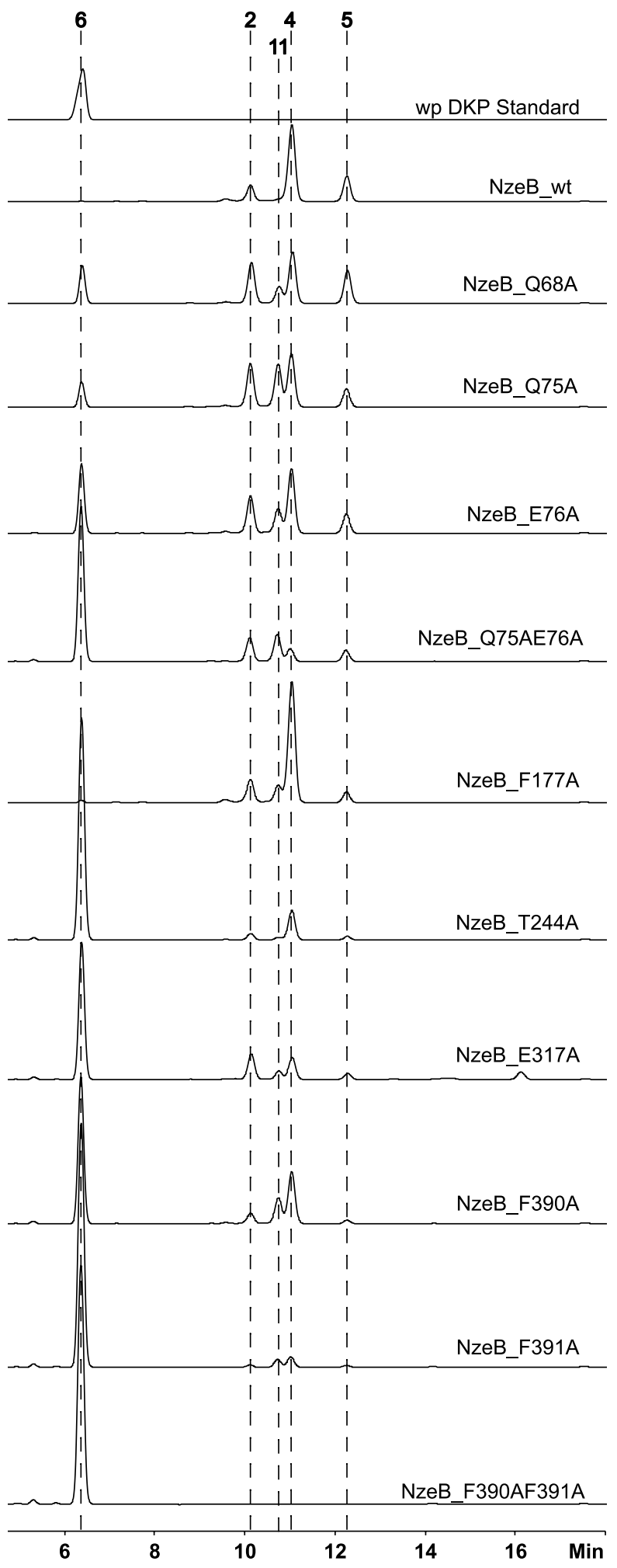


Supplementary Figure 14. ${ }^{1} \mathrm{H}$ NMR spectra for isolated (-)-aspergilazine A (5) from NzeB scale up reaction

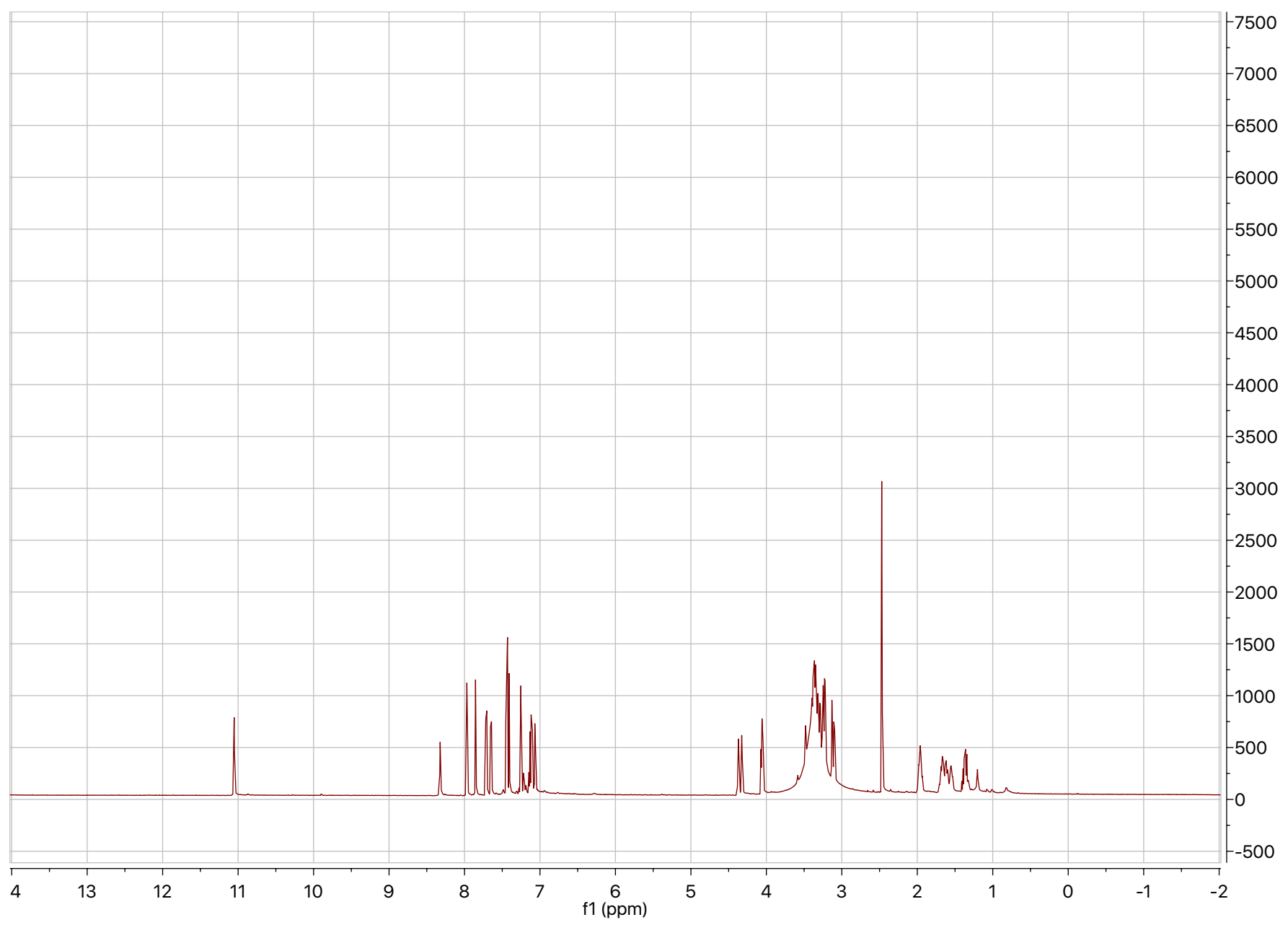

${ }^{1} \mathrm{H}$ NMR $\left.(599 \mathrm{MHz} \text {, DMSO-d })_{6}\right) 11.05(\mathrm{~s}, 1 \mathrm{H}), 7.97(\mathrm{~s}, 1 \mathrm{H}), 7.85(\mathrm{~s}, 1 \mathrm{H}), 7.71(\mathrm{~d}, J=8.3 \mathrm{~Hz}, 1 \mathrm{H}), 7.65(\mathrm{~d}, J=$ $7.8 \mathrm{~Hz}, 1 \mathrm{H}), 7.45(\mathrm{~d}, J=8.2 \mathrm{~Hz}, 1 \mathrm{H}), 7.43(\mathrm{~s}, 1 \mathrm{H}), 7.41(\mathrm{~d}, J=1.9 \mathrm{~Hz}, 1 \mathrm{H}), 7.25(\mathrm{~d}, J=2.4 \mathrm{~Hz}, 1 \mathrm{H}), 7.15-$ $7.12(\mathrm{~m}, 1 \mathrm{H}), 7.10(\mathrm{~d}, J=2.0 \mathrm{~Hz}, 1 \mathrm{H}), 7.07(\mathrm{t}, J=7.4 \mathrm{~Hz}, 1 \mathrm{H}), 4.37(\mathrm{t}, J=5.4 \mathrm{~Hz}, 1 \mathrm{H}), 4.33(\mathrm{t}, J=5.3 \mathrm{~Hz}, 1 \mathrm{H})$, $4.09-4.03(\mathrm{~m}, 2 \mathrm{H}), 3.35$ (tdd, $J=19.4,9.6,6.3 \mathrm{~Hz}, 3 \mathrm{H}), 3.30-3.20(\mathrm{~m}, 3 \mathrm{H}), 3.15-3.09(\mathrm{~m}, 2 \mathrm{H}), 1.96$ (dtt, $J$ $=14.0,6.9,3.5 \mathrm{~Hz}, 2 \mathrm{H}), 1.75-1.51(\mathrm{~m}, 4 \mathrm{H}), 1.36$ (ddtd, $J=18.1,12.4,10.2,7.8 \mathrm{~Hz}, 2 \mathrm{H})$. 
Supplementary Figure 15. ${ }^{13} \mathrm{C}$ NMR spectra for isolated (-)-aspergilazine A (5) from NzeB scale up reaction

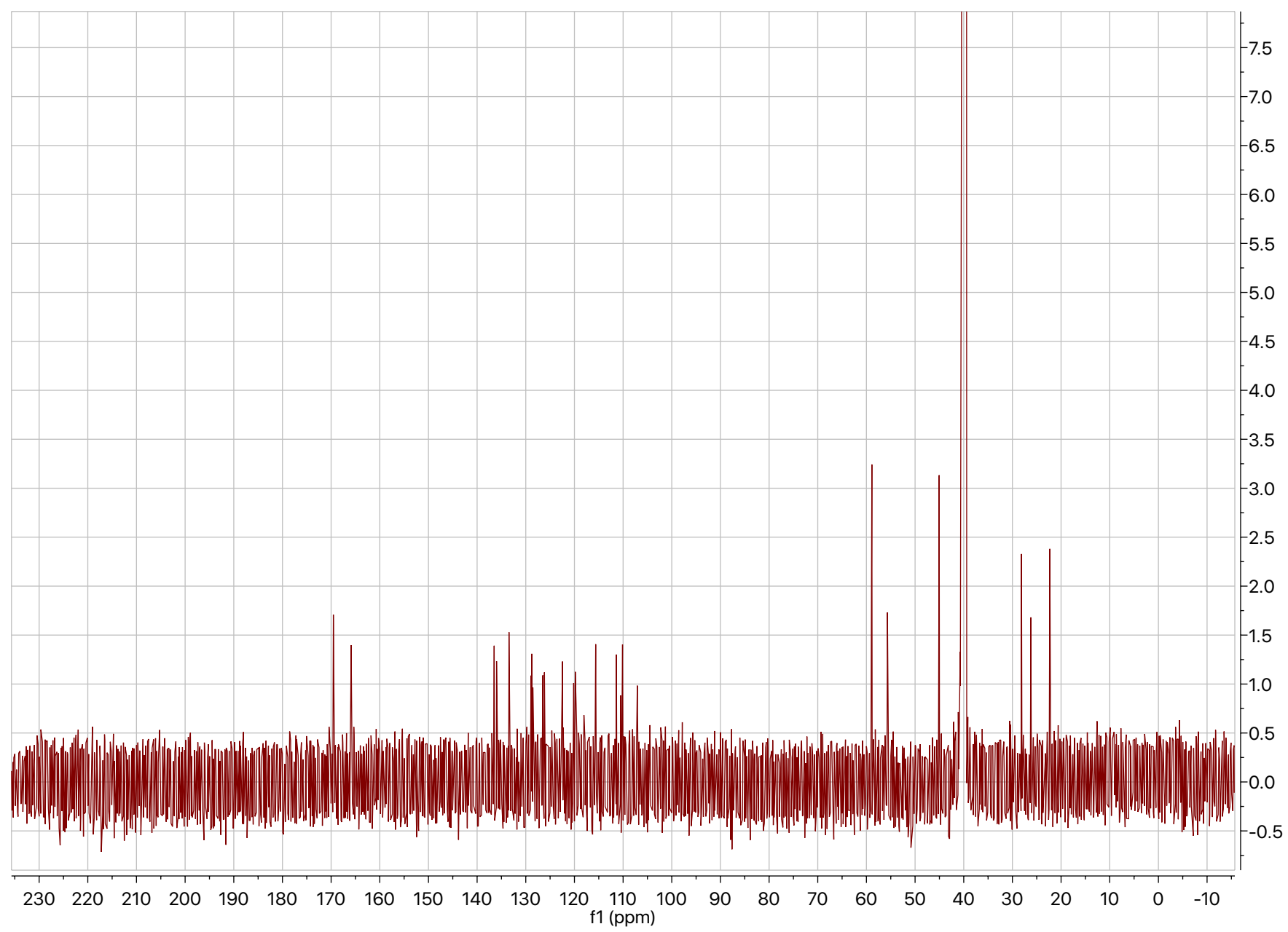

${ }^{13} \mathrm{C}$ NMR (151 MHz, DMSO-d6) $\delta 169.54,169.47,165.88,165.84,136.49,136.01,133.45,128.94,128.76$, $128.54,126.49,126.17,122.45,119.82,115.63,111.38,110.12,107.09,58.88,55.68,55.59,45.05,28.14$, $26.20,22.31,22.28$. 
Supplementary Figure 16. ${ }^{1} \mathrm{H}$ NMR spectra for 18 isolated from NzeB scale up

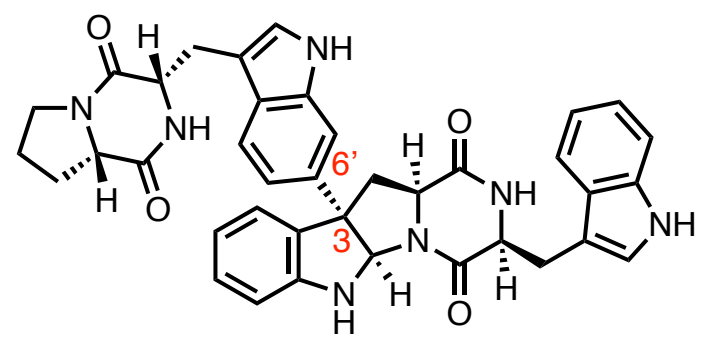

18

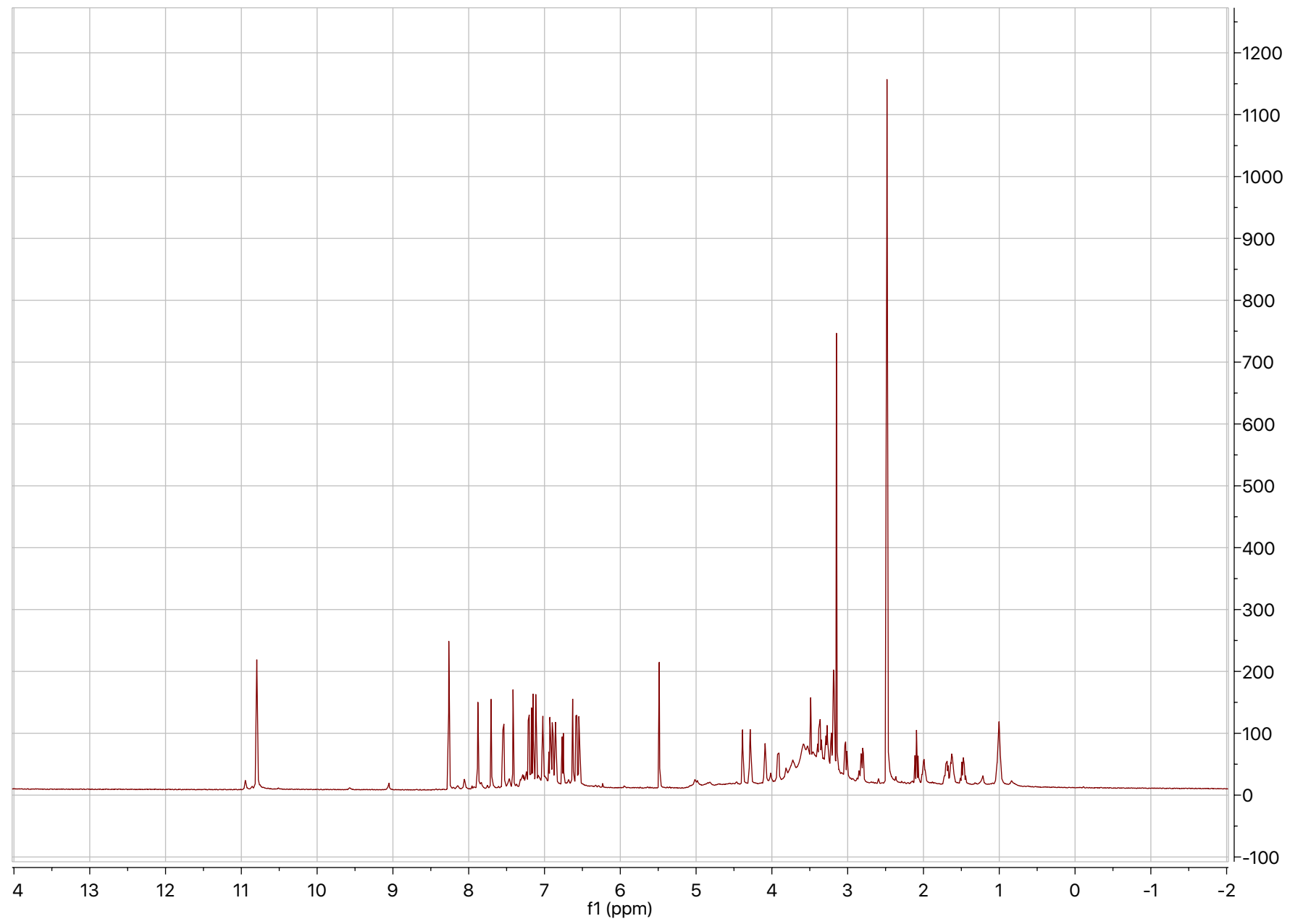

${ }^{1} \mathrm{H}$ NMR $\left(599 \mathrm{MHz}\right.$, DMSO-d $\left.\mathrm{d}_{6}\right) \delta 10.80(\mathrm{~s}, 2 \mathrm{H}), 8.26(\mathrm{~s}, 1 \mathrm{H}), 7.88(\mathrm{~s}, 1 \mathrm{H}), 7.70(\mathrm{~s}, 1 \mathrm{H}), 7.54(\mathrm{~d}, J=7.8 \mathrm{~Hz}, 1 \mathrm{H})$, $7.41(\mathrm{~s}, 1 \mathrm{H}), 7.19(\mathrm{dd}, J=17.9,8.2 \mathrm{~Hz}, 2 \mathrm{H}), 7.15(\mathrm{~d}, J=2.2 \mathrm{~Hz}, 1 \mathrm{H}), 7.12(\mathrm{~d}, J=2.3 \mathrm{~Hz}, 1 \mathrm{H}), 7.03(\mathrm{~d}, J=7.4$ $\mathrm{Hz}, 1 \mathrm{H}), 6.93(\mathrm{t}, J=7.6 \mathrm{~Hz}, 1 \mathrm{H}), 6.90(\mathrm{t}, J=7.5 \mathrm{~Hz}, 1 \mathrm{H}), 6.86(\mathrm{t}, J=7.4 \mathrm{~Hz}, 1 \mathrm{H}), 6.76(\mathrm{dd}, J=8.5,1.7 \mathrm{~Hz}, 1 \mathrm{H})$, $6.63(\mathrm{~s}, 1 \mathrm{H}), 6.58(\mathrm{~d}, J=7.8 \mathrm{~Hz}, 1 \mathrm{H}), 6.55(\mathrm{t}, J=7.4 \mathrm{~Hz}, 1 \mathrm{H}), 5.49(\mathrm{~s}, 1 \mathrm{H}), 4.38(\mathrm{~d}, J=5.1 \mathrm{~Hz}, 1 \mathrm{H}), 4.29(\mathrm{t}, J=$ $5.5 \mathrm{~Hz}, 1 \mathrm{H}), 4.12-4.06(\mathrm{~m}, 1 \mathrm{H}), 3.92(\mathrm{dd}, J=11.8,5.5 \mathrm{~Hz}, 1 \mathrm{H}), 3.37(\mathrm{dt}, J=11.7,7.9 \mathrm{~Hz}, 1 \mathrm{H}), 3.27(\mathrm{ddd}, J=$ $12.2,8.8,3.9 \mathrm{~Hz}, 1 \mathrm{H}), 3.23-3.17(\mathrm{~m}, 3 \mathrm{H}), 3.02(\mathrm{dd}, J=15.0,6.0 \mathrm{~Hz}, 1 \mathrm{H}), 2.81(\mathrm{dd}, J=12.2,5.6 \mathrm{~Hz}, 1 \mathrm{H})$, $2.09(\mathrm{t}, J=11.9 \mathrm{~Hz}, 1 \mathrm{H}), 2.04-1.95(\mathrm{~m}, 1 \mathrm{H}), 1.73-1.58(\mathrm{~m}, 2 \mathrm{H}), 1.53-1.42(\mathrm{~m}, 1 \mathrm{H})$. 
Supplementary Figure 17. ${ }^{13} \mathrm{C}$ NMR spectra for 18 isolated from NzeB scale up

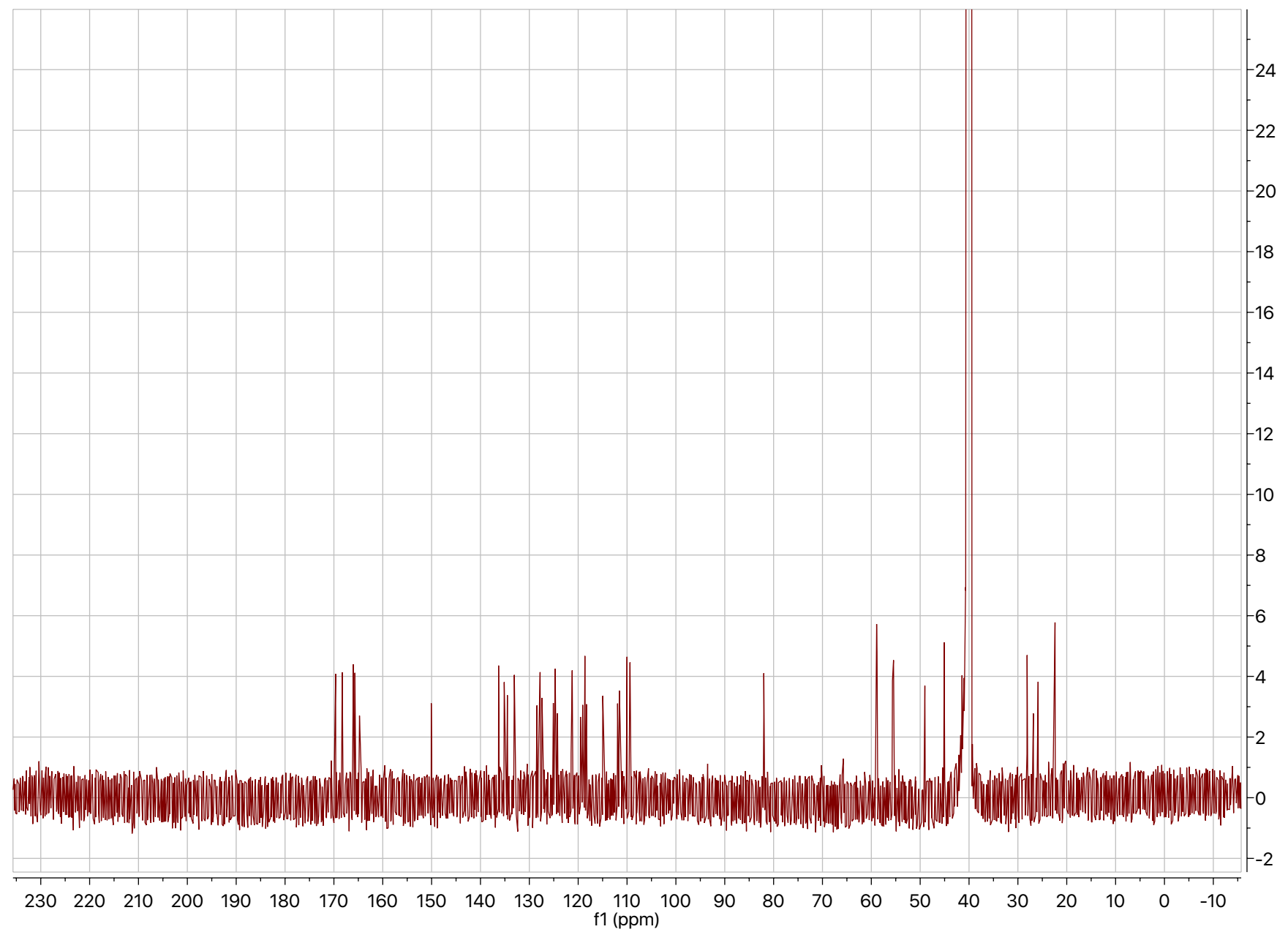

${ }^{13} \mathrm{C}$ NMR (151 MHz, dmso) $\delta 169.64,168.30,166.01,165.70,164.76,150.07,136.29,135.14,134.50,133.07$, $128.45,127.79,127.40,125.08,124.71,124.24,121.22,119.53,119.08,118.63,118.31,114.97,111.94$, $111.57,110.00,109.45,109.39,82.00,59.02,58.91,58.83,55.68,55.45,49.04,45.09,41.43,28.11,26.83$, 25.86, 22.40 . 
Supplementary Figure 18. gHSQCAD spectra for 18 isolated from NzeB scale up

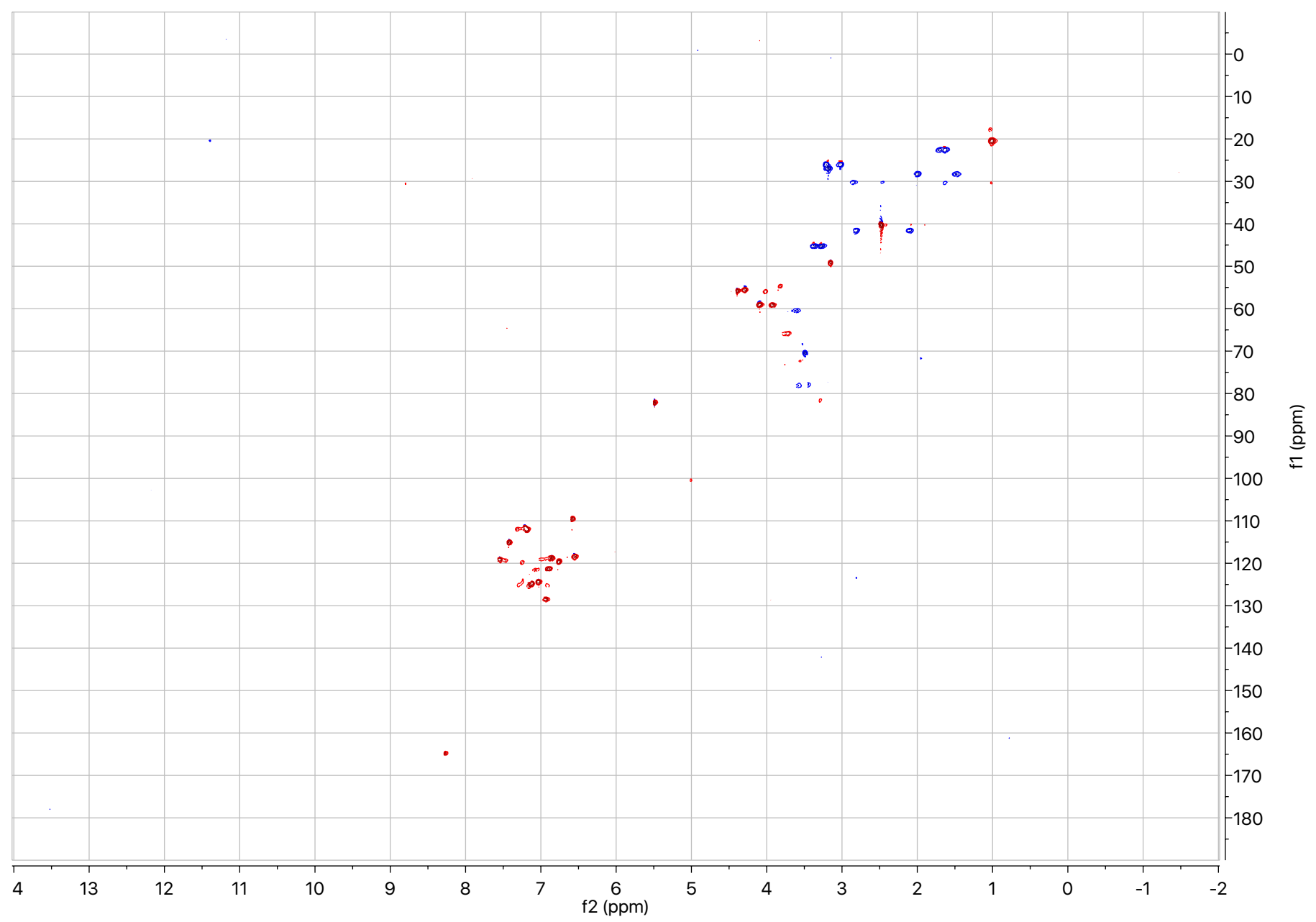


Supplementary Figure 19. gHMBCAD spectra for 18 isolated from NzeB scale up

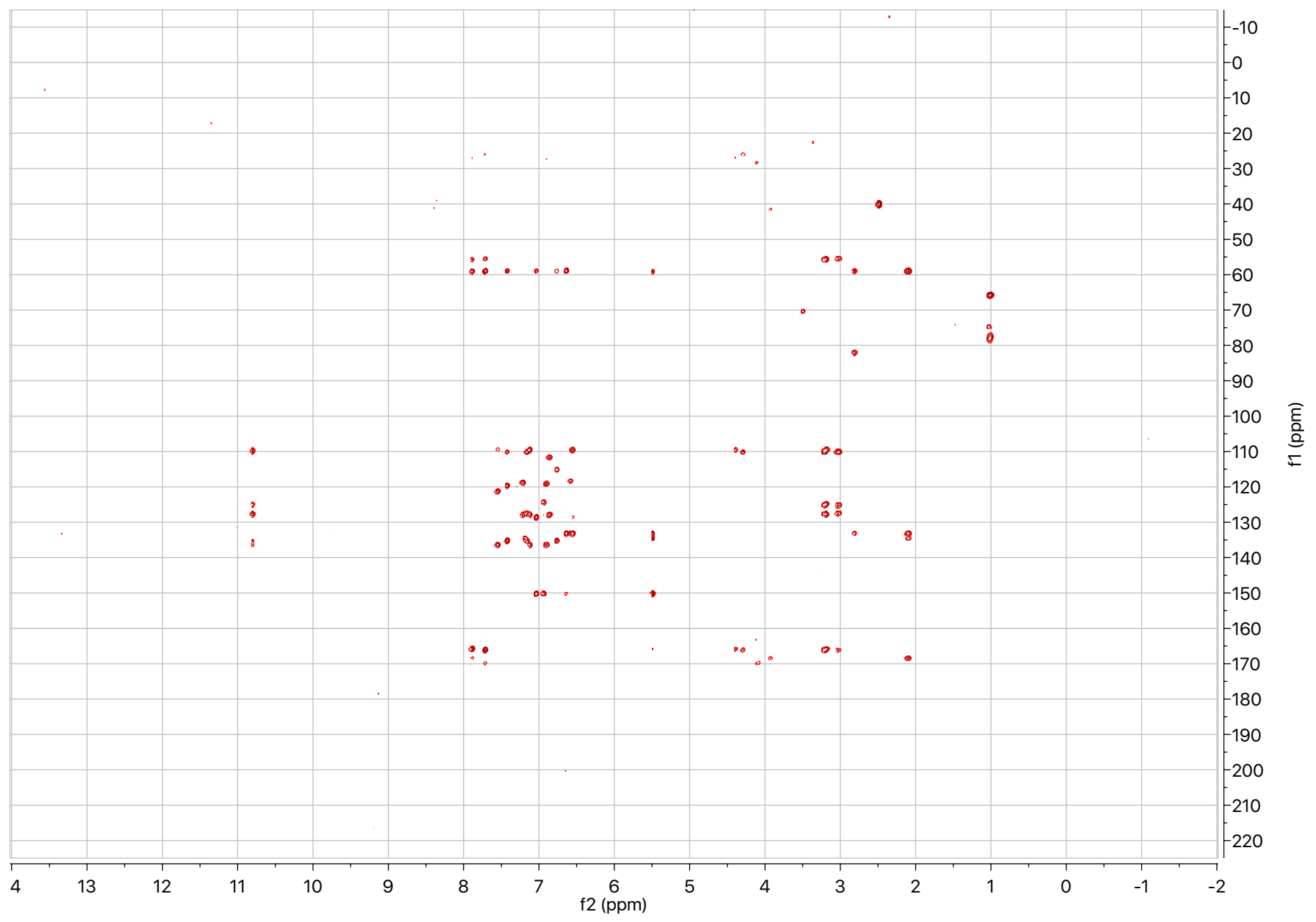


Supplementary Figure 20. gCOSY spectra for 18 isolated from NzeB scale up

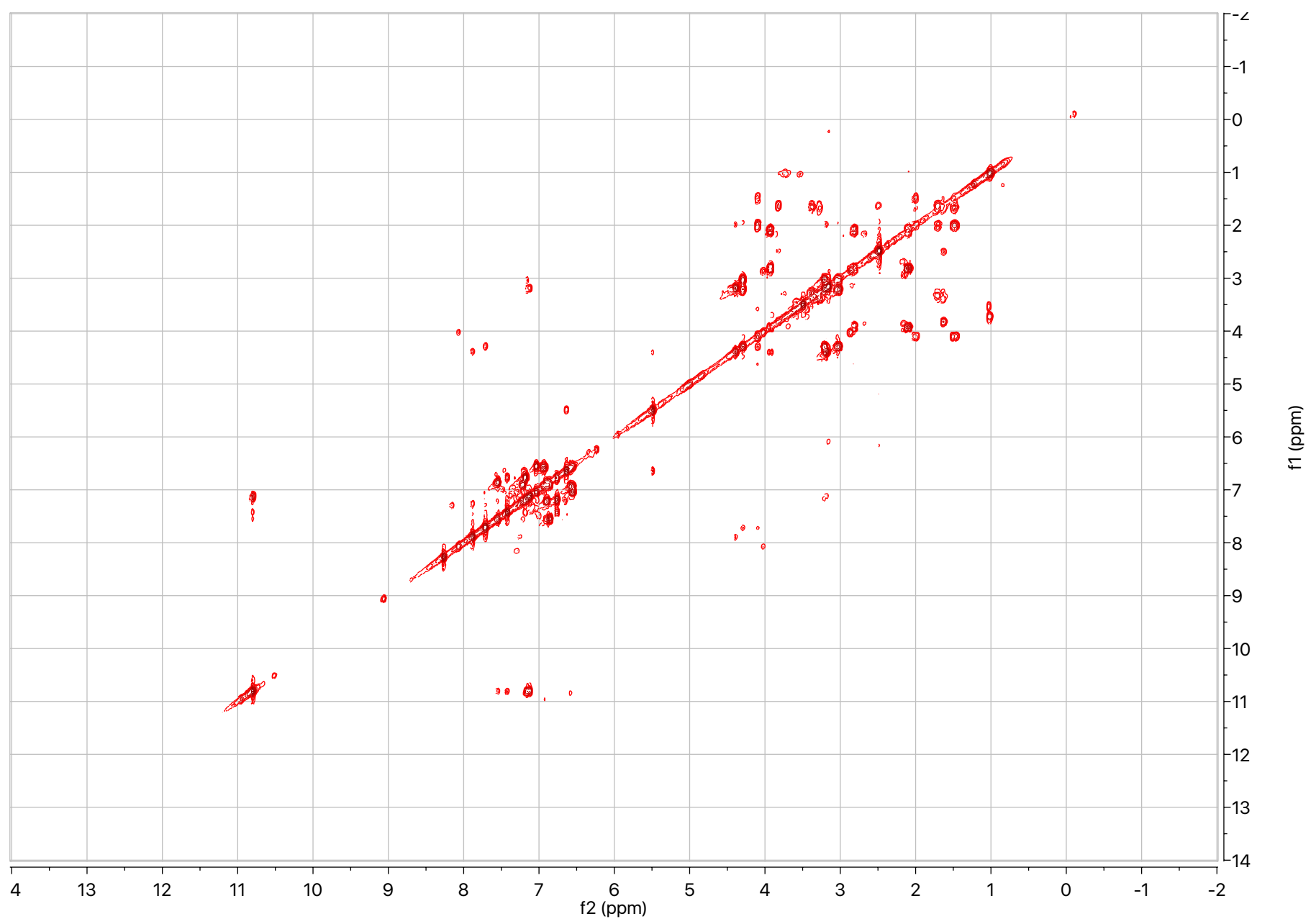


Supplementary Table 3. Cytochromes P450 used to generate sequence similarity network (SSN)

\begin{tabular}{|c|c|c|c|c|}
\hline P450 & Organism & Accession & Natural Product & Reference \\
\hline NascB & $\begin{array}{c}\text { Streptomyces } \\
\text { sp.CMB-MQ030 }\end{array}$ & AWF71596.1 & (-)-naseseazine C & Nat. Commun. 2018, 4428 \\
\hline P450NB5737 & $\begin{array}{c}\text { S. purpureus } \\
\text { (NRRL B-5737) }\end{array}$ & WP_019889608 & guanitrypmycin C3-1 & Org. Lett. 2018, 4921 \\
\hline TxtC & S. scabies & 6F0C_A & thaxtomin A & JACS 2019, 216 \\
\hline CypX & B. subtilis & WP_148962661. & pulcherrimic acid & Biochemistry, 2010, 7282 \\
\hline BcmD & $\begin{array}{c}\text { S. } \\
\text { sapporonensis }\end{array}$ & AXQ04975.1 & bicyclomycin & ACIEE 2018, 719 \\
\hline CYP121 & M. tuberculosis & P9WPP7.1 & mycocyclosin & J. Biol. Chem. 2003, 5141 \\
\hline
\end{tabular}




\section{Process of crystallization}

Crystallization of NzeB.

Single, diffraction quality crystals of the $\mathrm{NzeB}$ complexes were grown by sitting drop vapor diffusion at $20^{\circ} \mathrm{C}$ by mixing $2 \mu \mathrm{L}$ of $8 \mathrm{mg} / \mathrm{mL}$ protein containing $1 \mathrm{mM} \mathrm{DKP}$ substrate and $0.2 \%$ DMSO with $2 \mu \mathrm{L}$ of a well solution containing 23\% PEG 3350, $100 \mathrm{mM}$ DL-malic acid, 2.5\% ethylene glycol. Equimolar concentrations of substrate (0.5 mM) were used for the NzeB.6.17 complex. Sitting droplets were nucleated after $4 \mathrm{~h}$ from an earlier spontaneous crystallization using a cat whisker. Single crystals grew after 48 hours. $8 \mu \mathrm{L}$ of a cryoprotecting solution containing $10 \mathrm{mM}$ Tris $\mathrm{pH}$ 7.5, 2 \% glycerol, 23\% PEG 3350, $100 \mathrm{mM}$ DL-malic acid, 18\% ethylene glycol, $1 \mathrm{mM}$ DKP substrate, $0.2 \%$ DMSO was added directly to the sitting drops and the crystals were harvested using nylon loops and vitrified by rapid plunging into liquid nitrogen. $N z e B$ crystallized in space group $P$ 1 with unit cell dimensions of $a=56.1 \AA, b=56.3 \AA, c=58.8 \AA, \alpha=91.1^{\circ}, \beta=92.9^{\circ}, y=100.1^{\circ}$ and two chains in the asymmetric unit.

Data collection and processing.

X-ray data were collected at $100 \mathrm{~K}$ on beamline 23ID-B at the National Institute for General Medical Sciences (NIGMS) and National Cancer Institute (NCI) Structural Biology Facility at the Advanced Photon Source in Argonne, IL, USA. Diffraction data were integrated and scaled using XDS. ${ }^{1}$ Data collection statistics are given in Supplementary Table 2.

Molecular replacement, model building and refinement.

The structure of NascB-F5053 was solved using Phenix MR-Rosetta. ${ }^{2}$ This resulted in an initial model that could be extended by alternating cycles of manual building in $\operatorname{Coot}^{3}$ and least-squares refinement with Phenix. ${ }^{4}$ Final models were validated using MolProbity. ${ }^{5}$ 
Supplementary Table 4. Data Collection Statistics

\begin{tabular}{|c|c|c|c|}
\hline & NzeB in complex with 6 & $\begin{array}{l}\text { NzeB in complex with } 6 \text { and } \\
\mathbf{1 7}\end{array}$ & NzeB ligand free \\
\hline Wavelength $(\AA)$ & 1.03 & 1.03 & 0.826 \\
\hline Resolution range $(\AA)$ & $\begin{array}{l}40.83-1.49 \\
(1.54-1.49)\end{array}$ & $\begin{array}{l}41.22-1.48 \\
(1.53-1.48)\end{array}$ & $\begin{array}{l}39.13-1.498 \\
(1.55-1.50)\end{array}$ \\
\hline Space group & $\mathrm{P} 1$ & $\mathrm{P} 1$ & $\mathrm{P} 22_{1}$ \\
\hline Unit cell & $\begin{array}{l}56.12,56.28,58.78 \AA \\
91.13,92.93,100.10^{\circ} \\
\end{array}$ & \begin{tabular}{|l}
$56.07,56.13,58.48 \AA$ \\
$91.36,93.04,101.10^{\circ}$ \\
\end{tabular} & $\begin{array}{l}55.71,55.81,56.10 \AA \\
90,101.92,90^{\circ}\end{array}$ \\
\hline Total reflections & 382537 (31077) & $324387(4806)$ & 359895 (35646) \\
\hline Unique reflections & $109037(10046)$ & $94423(2902)$ & $54058(5282)$ \\
\hline Multiplicity & $3.5(3.1)$ & $3.4(1.7)$ & $6.7(6.7)$ \\
\hline Completeness (\%) & $94.48(87.34)$ & $80.96(24.95)$ & $99.66(97.45)$ \\
\hline Mean I/sigma(I) & $16.78(2.39)$ & $20.64(1.81)$ & $14.45(1.35)$ \\
\hline Wilson B-factor $(\AA 2)$ & 19.96 & 22.24 & 23.40 \\
\hline R-merge & $0.039(0.361)$ & $0.030(0.287)$ & $0.056(1.013)$ \\
\hline R-meas & $0.046(0.439)$ & $0.036(0.398)$ & $0.061(1.097)$ \\
\hline $\mathrm{CC} 1 / 2$ & $0.998(0.85)$ & $0.999(0.851)$ & $0.999(0.743)$ \\
\hline $\begin{array}{l}\text { Reflections used in } \\
\text { refinement }\end{array}$ & $109025(10043)$ & $94401(2902)$ & 54024 (5279) \\
\hline R-work & $0.168(0.287)$ & $0.192(0.333)$ & $0.188(0.292)$ \\
\hline R-free & $0.211(0.340)$ & $0.234(0.410)$ & $0.219(0.307)$ \\
\hline $\begin{array}{l}\text { Number of atoms } \\
\text { (total): }\end{array}$ & 6989 & 6995 & 3441 \\
\hline macromolecules & 6108 & 6079 & 3021 \\
\hline ligands & 190 & 196 & 55 \\
\hline solvent & 691 & 720 & 365 \\
\hline Protein residues & 798 & 793 & 392 \\
\hline RMS(bonds) & 0.005 & 0.007 & 0.006 \\
\hline RMS(angles) & 0.91 & 1.23 & 0.84 \\
\hline $\begin{array}{l}\text { Ramachandran } \\
\text { favored }(\%)\end{array}$ & 97.21 & 97.56 & 97.91 \\
\hline $\begin{array}{l}\text { Ramachandran } \\
\text { allowed (\%) }\end{array}$ & 2.79 & 2.44 & 2.09 \\
\hline $\begin{array}{l}\text { Ramachandran } \\
\text { outliers }(\%) \\
\end{array}$ & 0.00 & 0.00 & 0.00 \\
\hline $\begin{array}{l}\text { Average B-factor } \\
(\AA 2)\end{array}$ & 27.51 & 28.55 & 31.52 \\
\hline macromolecules & 26.79 & 28.07 & 30.84 \\
\hline ligands & 19.50 & 22.09 & 25.73 \\
\hline solvent & 36.01 & 34.38 & 37.97 \\
\hline
\end{tabular}


Supplementary Figure 21. Comparison of A) NzeB substrate bound, and B) substrate free active sites.

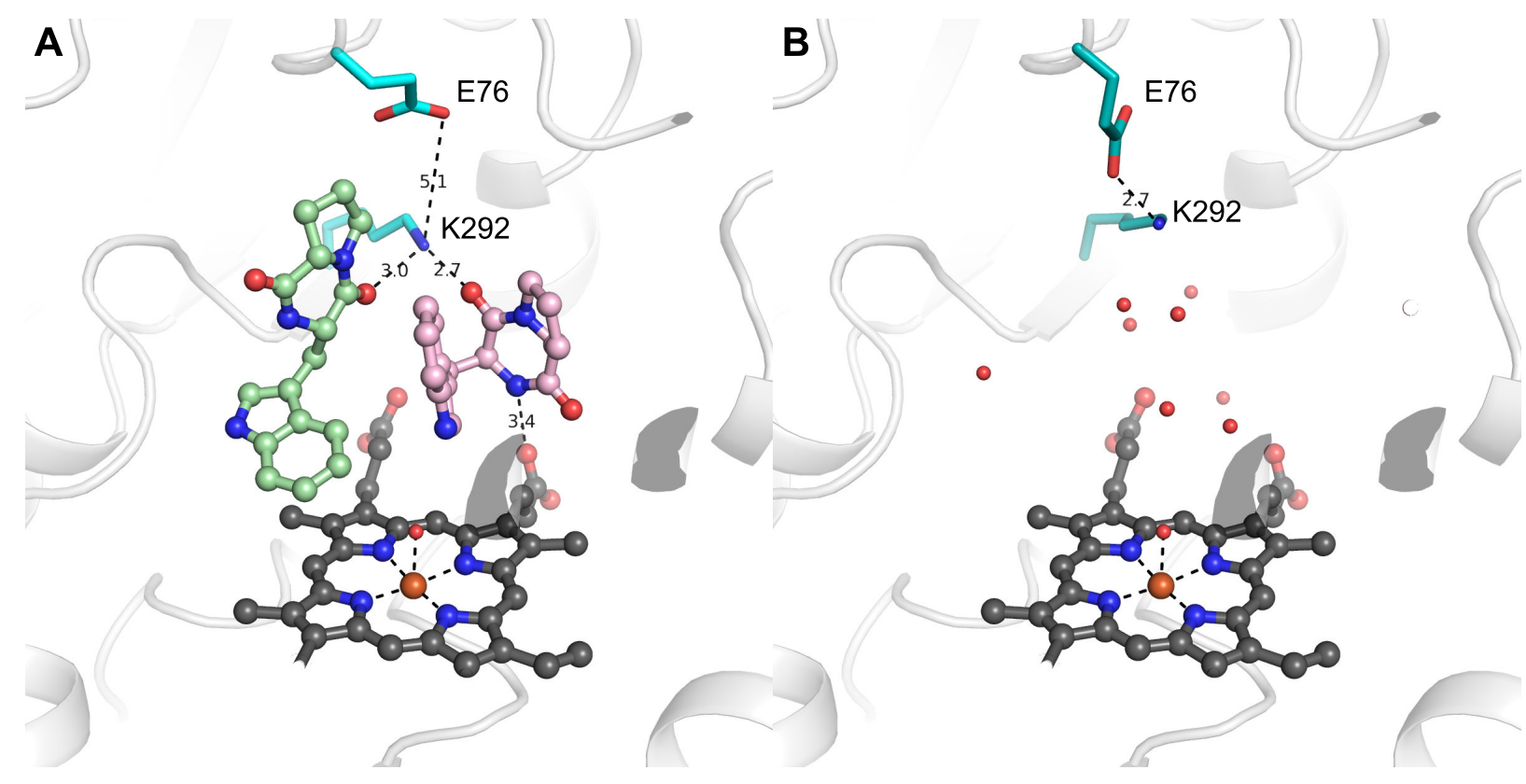


Supplementary Figure 22. NzeB Active site showing A) steric packing in dimerization site, b) open channel in cyclization site, C) and the view from $180^{\circ}$ rotation.

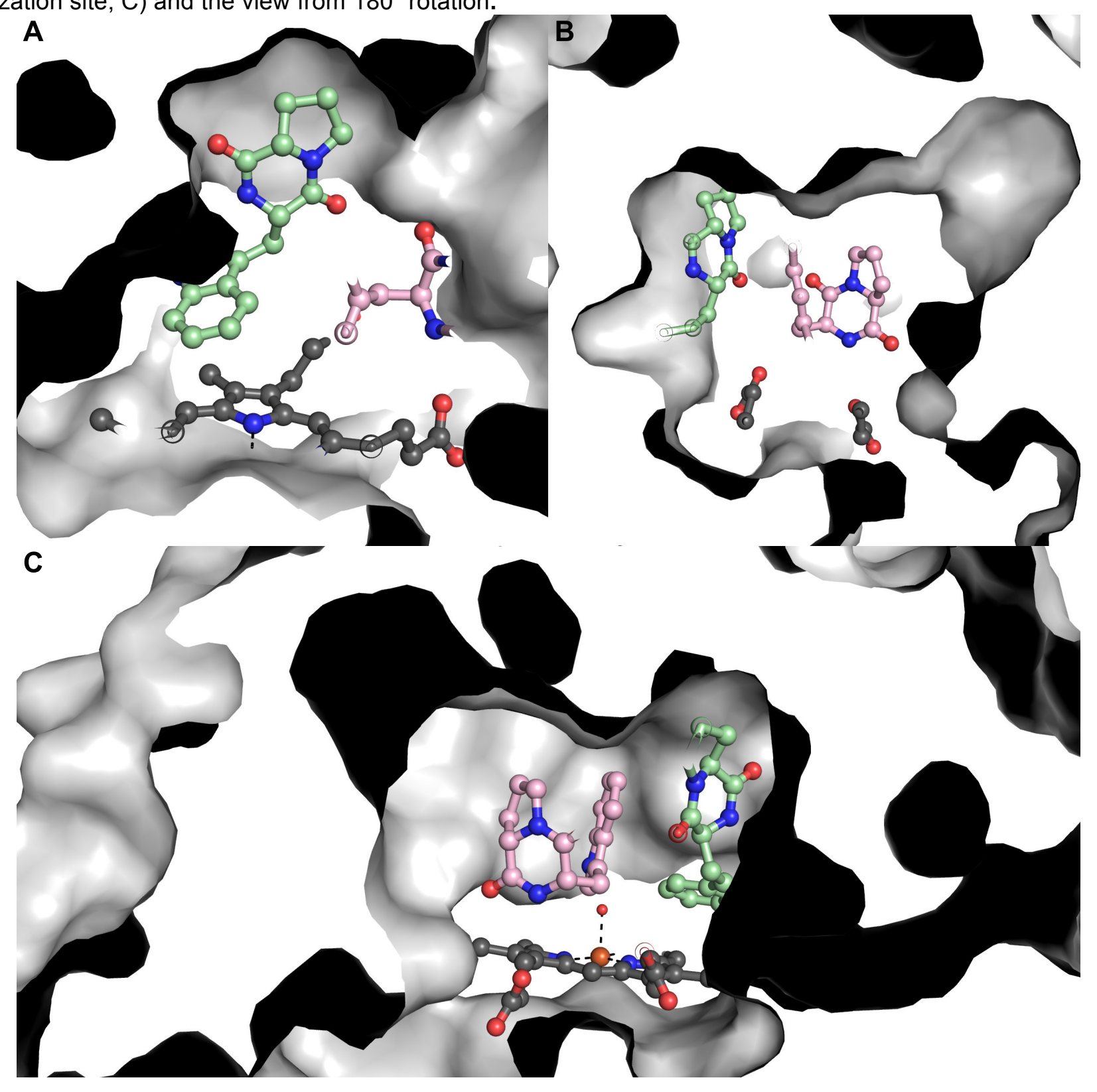




\section{Crystallography References:}

1. Kabsch, W., Integration, scaling, space-group assignment and post-refinement. Acta Crystallogr. D Biol. Crystallogr. 2010, 66 (Pt 2), 133-44.

2. Terwilliger, T. C.; Dimaio, F.; Read, R. J.; Baker, D.; Bunkoczi, G.; Adams, P. D.; Grosse-Kunstleve, R. W.; Afonine, P. V.; Echols, N., phenix.mr_rosetta: molecular replacement and model rebuilding with Phenix and Rosetta. J. Struct. Funct. Genomics 2012, 13 (2), 81-90.

3. Emsley, P.; Cowtan, K., Coot: model-building tools for molecular graphics. Acta Crystallogr. D Biol. Crystallogr. 2004, 60 (Pt 12 Pt 1), 2126-32.

4. Adams, P. D.; Afonine, P. V.; Bunkoczi, G.; Chen, V. B.; Davis, I. W.; Echols, N.; Headd, J. J.; Hung, L. W.; Kapral, G. J.; Grosse-Kunstleve, R. W.; McCoy, A. J.; Moriarty, N. W.; Oeffner, R.; Read, R. J.; Richardson, D. C.; Richardson, J. S.; Terwilliger, T. C.; Zwart, P. H., PHENIX: a comprehensive Python-based system for macromolecular structure solution. Acta Crystallogr. D Biol. Crystallogr. 2010, 66 (Pt 2), 213-21.

5. Chen, V. B.; Arendall, W. B., 3rd; Headd, J. J.; Keedy, D. A.; Immormino, R. M.; Kapral, G. J.; Murray, L. W.; Richardson, J. S.; Richardson, D. C., MolProbity: all-atom structure validation for macromolecular crystallography. Acta Crystallogr. D Biol. Crystallogr. 2010, 66 (Pt 1), 12-21. 


\section{Computational Methods}

Conformational searches were performed using the Schrödinger MacroModel ${ }^{1}$ software package to identify low-energy conformers for quantum mechanical calculations. All quantum mechanical calculations were performed using the Gaussian $09^{2}$ software package. Structures were optimized at the UB3LYP $P^{3,4} /$ LanL2DZ(Fe $)^{5} / 6-31 G(d)$ level of theory using the CPCM $^{6-8}$ solvent model for diethyl ether, which is intended to model the environment of the enzyme active site; frequency calculations were used to confirm the presence of local minima (no imaginary frequencies) and transition states (one imaginary frequency). Thermochemistry corrections to obtain enthalpies and free energies were computed at $298 \mathrm{~K}$ and $1 \mathrm{M}$, with Truhlar corrections ${ }^{9}$ applied to all frequencies below $100 \mathrm{~cm}^{-1}$. To obtain more accurate energetics, singlepoint energy calculations were performed on the optimized structures at the UB3LYP ${ }^{3,4} / \mathrm{LanL} 2 \mathrm{DZ}(\mathrm{Fe})^{5} / 6$ $311++G(2 d, 2 p)$ level of theory using Grimme's D3(BJ) dispersion correction ${ }^{10,11}$ and the $\mathrm{CPCM}^{6-8}$ solvent model for diethyl ether. For all iron-containing species, all possible spin states were computed separately; the spin state with the lowest free energy is reported and used in the computed reaction pathways. 
Supplementary Table 5: Optimized Quantum Mechanical Energies and Geometries

\begin{tabular}{|c|c|c|c|}
\hline Structure & $\begin{array}{c}\text { Single-Point Energy } \\
\text { (Hartree) } \\
\text { UB3LYP/ } \\
6-311++G(2 d, 2 p) \\
\text { CPCM diethylether solvent } \\
\text { D3-BJ dispersion correction }\end{array}$ & $\begin{array}{c}\text { Enthalpy Correction } \\
\text { (Hartree) } \\
\text { UB3LYP/6-31G(d) } \\
\text { CPCM diethylether } \\
\text { solvent }\end{array}$ & $\begin{array}{c}\begin{array}{c}\text { Gibbs Free } \\
\text { Energy Correction } \\
\text { (Hartree) }\end{array} \\
\text { UB3LYP/6-31G(d) } \\
\text { CPCM diethylether } \\
\text { solvent }\end{array}$ \\
\hline Compound 6 & -935.064995353 & 0.332193 & 0.267005 \\
\hline Compound PC-I & -2560.69811814 & 0.675898 & 0.552976 \\
\hline Transition State from PC-I to R-I & -2560.68200451 & 0.668580 & 0.548785 \\
\hline Compound R-I & -934.408484774 & 0.318396 & 0.252323 \\
\hline Compound PC-II & -2560.69111264 & 0.675486 & 0.550891 \\
\hline Transition State from PC-II to R-II & -2560.66865641 & 0.668467 & 0.549941 \\
\hline Compound R-II & -934.378337774 & 0.317810 & 0.251336 \\
\hline Transition State from $\mathbf{R}$-II to $\mathbf{R}$-III & -934.375022742 & 0.317227 & 0.254810 \\
\hline Compound R-III & -934.406015167 & 0.319252 & 0.257416 \\
\hline 3-methylindole & -403.297212617 & 0.166766 & 0.125308 \\
\hline Transition State from $\mathbf{R}$-III to $\mathbf{R}$-IV & -1337.68979078 & 0.486702 & 0.403190 \\
\hline Compound R-IV & -1337.70558024 & 0.488372 & 0.406134 \\
\hline Compound PDT $_{\mathrm{C}-\mathrm{C}}$ & -1337.15954501 & 0.479009 & 0.398229 \\
\hline Compound C-III & -934.228902694 & 0.321380 & 0.260447 \\
\hline Transition State from $\mathbf{C}$-III to $\mathbf{C}$-IV & -1337.54044714 & 0.489605 & 0.408473 \\
\hline Compound C-IV & -1337.55215821 & 0.491575 & 0.410726 \\
\hline Compound R-V & -934.408292246 & 0.318638 & 0.253313 \\
\hline Transition State from $\mathbf{R}-\mathbf{V}$ to $\mathbf{R}-\mathbf{V} \mathbf{I}$ & -1337.69321370 & 0.486722 & 0.403322 \\
\hline Compound R-VI & -1337.70428197 & 0.488290 & 0.400752 \\
\hline Compound DDT $_{\mathrm{C}-\mathrm{N}}$ & -1337.15657933 & 0.478377 & 0.394108 \\
\hline Compound C-V & -934.179753468 & 0.319454 & 0.254095 \\
\hline Iron-Oxo Quartet & -1625.60979018 & 0.341464 & 0.266017 \\
\hline Iron-Hydroxo Triplet & -1626.26453700 & 0.352745 & 0.277069 \\
\hline Iron-Aqua Doublet & -1626.92110182 & 0.365210 & 0.288026 \\
\hline Iron-Hydroxo Doublet Anion & -1626.42321553 & 0.351663 & 0.276113 \\
\hline Figure 11B Radical C2 TS & -1337.69755972 & 0.486871 & 0.403638 \\
\hline Figure 11B Radical C2 Product & -1337.72340783 & 0.489309 & 0.407811 \\
\hline Figure 11B Radical C3 TS & -1337.69205782 & 0.486851 & 0.405608 \\
\hline Figure 11B Radical C3 Product & -1337.70825431 & 0.488798 & 0.408087 \\
\hline Figure 11B Radical C4 TS & -1337.69550474 & 0.487160 & 0.405177 \\
\hline Figure 11B Radical C4 Product & -1337.71350934 & 0.489101 & 0.407451 \\
\hline Figure 11B Radical C5 TS & -1337.68979078 & 0.486702 & 0.403190 \\
\hline Figure 11B Radical C5 Product & -1337.70558024 & 0.488372 & 0.406134 \\
\hline Figure 11B Radical C6 TS & -1337.69174411 & 0.486788 & 0.403349 \\
\hline Figure 11B Radical C6 Product & -1337.70914756 & 0.488422 & 0.405258 \\
\hline Figure 11B Radical C7 TS & -1337.69379606 & 0.486938 & 0.404223 \\
\hline Figure 11B Radical C7 Product & -1337.71560829 & 0.489107 & 0.407992 \\
\hline Figure 11D Cation C2 TS & -1337.53832625 & 0.489255 & 0.407257 \\
\hline Figure 11D Cation C2 Product & -1337.56124772 & 0.491742 & 0.410737 \\
\hline Figure 11D Cation C3 TS & -1337.54174717 & 0.489798 & 0.408678 \\
\hline Figure 11D Cation C3 Product & -1337.56290761 & 0.492522 & 0.413750 \\
\hline
\end{tabular}




\begin{tabular}{|c|c|c|c|}
\hline Figure 11D Cation C4 TS & -1337.53534315 & 0.489690 & 0.408765 \\
\hline Figure 11D Cation C4 Product & -1337.54696654 & 0.492005 & 0.412079 \\
\hline Figure 11D Cation C5 TS & -1337.54044714 & 0.489605 & 0.408473 \\
\hline Figure 11D Cation C5 Product & -1337.55215821 & 0.491575 & 0.410726 \\
\hline Figure 11D Cation C6 TS & -1337.53368503 & 0.489370 & 0.406633 \\
\hline Figure 11D Cation C6 Product & -1337.55077701 & 0.491474 & 0.409790 \\
\hline Figure 11D Cation C7 TS & -1337.53365020 & 0.489592 & 0.407774 \\
\hline Figure 11D Cation C7 Product & -1337.55229112 & 0.491566 & 0.411883 \\
\hline Figure 12B Radical C2 TS & -1337.69951164 & 0.486587 & 0.402050 \\
\hline Figure 12B Radical C2 Product & -1337.72283996 & 0.488652 & 0.402337 \\
\hline Figure 12B Radical C3 TS & -1337.69039795 & 0.486633 & 0.404156 \\
\hline Figure 12B Radical C3 Product & -1337.69932417 & 0.488295 & 0.403578 \\
\hline Figure 12B Radical C4 TS & -1337.69014921 & 0.486500 & 0.401321 \\
\hline Figure 12B Radical C4 Product & -1337.71217497 & 0.488517 & 0.401914 \\
\hline Figure 12B Radical C5 TS & -1337.68944354 & 0.486708 & 0.404293 \\
\hline Figure 12B Radical C5 Product & -1337.69958304 & 0.488216 & 0.400754 \\
\hline Figure 12B Radical C6 TS & -1337.69321370 & 0.486722 & 0.403322 \\
\hline Figure 12B Radical C6 Product & -1337.70428197 & 0.488290 & 0.400752 \\
\hline Figure 12B Radical C7 TS & -1337.68740315 & 0.486476 & 0.401229 \\
\hline Figure 12B Radical C7 Product & -1337.70835455 & 0.488657 & 0.402298 \\
\hline Compound SI-6 & -935.062969833 & 0.332324 & 0.268187 \\
\hline Compound SI-PC-I & -2560.69153790 & 0.675829 & 0.551602 \\
\hline TS from SI-PC-I to SI-R-I & -2560.67865279 & 0.668912 & 0.547686 \\
\hline Compound SI-R-I & -934.408292246 & 0.318638 & 0.253313 \\
\hline Compound SI-PC-II & -2560.69492543 & 0.676111 & 0.552240 \\
\hline TS from SI-PC-II to SI-R-II & -2560.66886329 & 0.668918 & 0.548679 \\
\hline Compound SI-R-II & -934.380566351 & 0.317339 & 0.251457 \\
\hline TS from SI-R-II to SI-R-III & -934.378935128 & 0.317125 & 0.255510 \\
\hline Compound SI-R-III & -934.402373050 & 0.319158 & 0.257062 \\
\hline TS from SI-R-III to SI-R-IV & -1337.68947287 & 0.487089 & 0.404197 \\
\hline Compound SI-R-IV & -1337.70630293 & 0.488419 & 0.405430 \\
\hline Compound SI-PDT ${ }_{C-C}$ & -1337.15713807 & 0.479196 & 0.399406 \\
\hline Compound SI-C-III & -934.227258143 & 0.321166 & 0.259968 \\
\hline TS from SI-C-III to SI-C-IV & -1337.53149035 & 0.489508 & 0.407077 \\
\hline Compound SI-C-IV & -1337.54947128 & 0.491664 & 0.410192 \\
\hline Figure S23B Radical C2 TS & -1337.69720677 & 0.486790 & 0.403187 \\
\hline Figure S23B Radical C2 Product & -1337.72387603 & 0.489129 & 0.407753 \\
\hline Figure S23B Radical C3 TS & -1337.69024969 & 0.486815 & 0.406062 \\
\hline Figure S23B Radical C3 Product & -1337.70583195 & 0.488790 & 0.407690 \\
\hline Figure S23B Radical C4 TS & -1337.69238834 & 0.487192 & 0.405409 \\
\hline Figure S23B Radical C4 Product & -1337.71039565 & 0.488908 & 0.407710 \\
\hline Figure S23B Radical C5 TS & -1337.68710983 & 0.486895 & 0.403027 \\
\hline Figure S23B Radical C5 Product & -1337.70169171 & 0.488268 & 0.405240 \\
\hline Figure S23B Radical C6 TS & -1337.68947287 & 0.487089 & 0.404197 \\
\hline Figure S23B Radical C6 Product & -1337.70630293 & 0.488419 & 0.405430 \\
\hline Figure S23B Radical C7 TS & -1337.69515416 & 0.487005 & 0.404216 \\
\hline Figure S23B Radical C7 Product & -1337.71296380 & 0.488933 & 0.406893 \\
\hline Figure S23D Cation C2 TS & -1337.53901559 & 0.489064 & 0.406499 \\
\hline Figure S23D Cation C2 Product & -1337.56397314 & 0.491670 & 0.411416 \\
\hline Figure S23D Cation C3 TS & -1337.54056391 & 0.489799 & 0.410016 \\
\hline Figure S23D Cation C3 Product & -1337.56254502 & 0.492561 & 0.414297 \\
\hline Figure S23D Cation C4 TS & -1337.53295258 & 0.489870 & 0.409363 \\
\hline
\end{tabular}




\begin{tabular}{|l|l|l|l|}
\hline Figure S23D Cation C4 Product & -1337.54486276 & 0.491909 & 0.412625 \\
\hline Figure S23D Cation C5 TS & -1337.53078689 & 0.489548 & 0.407453 \\
\hline Figure S23D Cation C5 Product & -1337.54732307 & 0.491534 & 0.410373 \\
\hline Figure S23D Cation C6 TS & -1337.53149035 & 0.489508 & 0.407077 \\
\hline Figure S23D Cation C6 Product & -1337.54947128 & 0.491664 & 0.410192 \\
\hline Figure S23D Cation C7 TS & -1337.53688422 & 0.489745 & 0.408768 \\
\hline Figure S23D Cation C7 Product & -1337.54891315 & 0.491855 & 0.410992 \\
\hline Compound 6 Radical Cation & -934.841312043 & 0.332396 & 0.265895 \\
\hline Iron-Oxo Anion Triplet & -1625.76714766 & 0.340805 & 0.266113 \\
\hline
\end{tabular}




$\begin{array}{lrrr}\text { Compound 6 } & & \\ 01 & & & \\ \text { N } & -3.499289 & 1.573570 & -1.088827 \\ \mathrm{C} & 1.839291 & -1.196192 & -0.541564 \\ \mathrm{O} & 1.826066 & -2.204846 & -1.249758 \\ \mathrm{C} & 0.556139 & -0.411010 & -0.221570 \\ \mathrm{C} & -5.029522 & 0.477437 & 0.591361 \\ \mathrm{C} & 3.030029 & 0.481835 & 0.883470 \\ \mathrm{C} & 4.482290 & 0.949079 & 0.719319 \\ \mathrm{C} & 5.247060 & -0.376807 & 0.556634 \\ \mathrm{C} & 4.296107 & -1.267749 & -0.264702 \\ \mathrm{C} & 1.970015 & 1.520484 & 0.536979 \\ \mathrm{C} & -0.483945 & -0.581218 & -1.349833 \\ \mathrm{C} & -1.795276 & 0.089217 & -1.056838 \\ \mathrm{C} & -2.259600 & 1.261651 & -1.608940 \\ \mathrm{C} & -3.868917 & 0.603390 & -0.181298 \\ \mathrm{C} & -2.815166 & -0.352831 & -0.133412 \\ \mathrm{C} & -2.948156 & -1.465888 & 0.717596 \\ \mathrm{C} & -4.099105 & -1.595235 & 1.485973 \\ \mathrm{C} & -5.128482 & -0.631631 & 1.424408 \\ \mathrm{~N} & 0.848386 & 1.009082 & -0.026565 \\ \mathrm{~N} & 2.965631 & -0.703387 & 0.015475 \\ \mathrm{O} & 2.130102 & 2.715542 & 0.776148 \\ \mathrm{H} & -4.050301 & 2.379233 & -1.344403 \\ \mathrm{H} & 0.136445 & -0.835920 & 0.704576 \\ \mathrm{H} & -5.823219 & 1.217901 & 0.540913 \\ \mathrm{H} & 2.854238 & 0.186240 & 1.931134 \\ \mathrm{H} & 4.820264 & 1.545090 & 1.569466 \\ \mathrm{H} & 4.567959 & 1.564711 & -0.182763 \\ \mathrm{H} & 5.429127 & -0.827695 & 1.538699 \\ \mathrm{H} & 6.214910 & -0.249410 & 0.064775 \\ \mathrm{H} & 4.335977 & -2.322526 & 0.023834 \\ \mathrm{H} & 4.493268 & -1.209952 & -1.341471 \\ \mathrm{H} & -0.615877 & -1.656822 & -1.501350 \\ \mathrm{H} & -0.054730 & -0.182839 & -2.276257 \\ \mathrm{H} & -1.805210 & 1.896742 & -2.357757 \\ \mathrm{H} & -2.165671 & -2.218741 & 0.770625 \\ \mathrm{H} & -4.211946 & -2.451727 & 2.145046 \\ \mathrm{H} & -6.016453 & -0.759741 & 2.037384 \\ \mathrm{H} & 0.082201 & 1.657848 & -0.179663\end{array}$

Compound PC-I

04
N -0.596448
C -6.297386
$\begin{array}{ll}1.739290 & -1.534251\end{array}$
O $\quad-6.959349$
$0.017667-0.842924$
C -4.919265
$0.271436-1.850792$
C -0.440828
$0.650980-0.599685$
C -5.986782
$4.164207-0.860686$
C -6.538474
$-1.152197$
1.353302
C -8.011209
$-2.534975$
1.726270
$-7.971361$
$-2.437386$
1.288243
C -4.477612
C -4.211008
C -2.845647
$-1.597063-0.002778$
$-1.0831051 .162991$
$0.972500-1.937796$
$\begin{array}{ll}1.566283 & -1.741575\end{array}$

\begin{tabular}{|c|c|c|c|}
\hline & 3315 & & \\
\hline & 174 & & \\
\hline & 932 & & \\
\hline & & & \\
\hline & & & \\
\hline & 0088 & 888 & \\
\hline & & 024 & \\
\hline & -6.7 & 292 & \\
\hline & -3.7 & 724 & \\
\hline & & 389 & \\
\hline & -5.0 & & -0.0 \\
\hline & 0.6 & 268 & \\
\hline & -6.24 & 9680 & 2.14667 \\
\hline & -6.4 & -2.7 & 2.78874 \\
\hline & -6.0 & 6060 & 1.15514 \\
\hline & -8.5 & -1.9 & 2.056020 \\
\hline & -8.4 & -3.4 & 1.125056 \\
\hline & -8.8 & -0.9 & -0.108017 \\
\hline & -7.9 & -2.2 & -0.905058 \\
\hline & -4.86 & 1.646328 & -2.495555 \\
\hline & -4.1 & 0.0 & -2.5 \\
\hline & & -0.1 & \\
\hline & -4.3 & 4.0 & -1.2 \\
\hline & -3.2 & & \\
\hline & -0.73 & 6.2 & $-0.33262 \varepsilon$ \\
\hline & -3.0 & -0.0 & 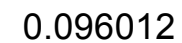 \\
\hline & & -0 . & -0 \\
\hline & 3.7 & -1. & -1 \\
\hline & & & -0 \\
\hline & & & \\
\hline & & 0.4 & \\
\hline & & -1.0 & -1 \\
\hline & -0. & & \\
\hline & & -2 & -2 \\
\hline & & -2 & \\
\hline & & & \\
\hline & & & \\
\hline & & & \\
\hline & & & 2.3 \\
\hline C & & -1.9 & -3.068861 \\
\hline S & -0.9 & -2.6 & 433 \\
\hline & & -2.7 & -3.233895 \\
\hline C & -0.2 & -3.2 & -1.023142 \\
\hline$C$ & & & 0.163457 \\
\hline & 0.22 & 708 & 3.153004 \\
\hline & 5.8 & 227 & 1.218017 \\
\hline & 1.31 & 899 & 3.348350 \\
\hline $\mathrm{H}$ & 6.19 & 3306 & -3.630102 \\
\hline 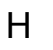 & -1.89 & 6697 & 0.411917 \\
\hline & 4.06 & 9828 & -3.96030 \\
\hline & $-0.6 c$ & -4.1 & -1.64188 \\
\hline & 7.43 & 108 & -0.23489 \\
\hline & -0.7 & & 36054 \\
\hline & & & 1.863 \\
\hline & 1.4 & 2.6 & 4.08494 \\
\hline
\end{tabular}




$\begin{array}{rrrr}\text { C } & 5.806708 & -0.095443 & -1.461884 \\ \mathrm{C} & -0.309839 & -0.793917 & 1.554543 \\ \mathrm{C} & 3.558668 & 2.031086 & 2.259988 \\ \mathrm{C} & 1.990300 & -3.005665 & -2.086647 \\ \mathrm{H} & 6.763308 & 0.033025 & -1.957923 \\ \mathrm{H} & -1.285266 & -0.910393 & 2.018369 \\ \mathrm{H} & 3.807964 & 2.828703 & 2.952438 \\ \mathrm{H} & 1.747310 & -3.817396 & -2.764801 \\ \mathrm{O} & 2.081031 & 0.735586 & -1.077832 \\ \mathrm{~S} & 4.000270 & -1.995224 & 1.498491 \\ \mathrm{C} & 3.902696 & -1.437322 & 3.226359 \\ \mathrm{H} & 2.866539 & -1.367596 & 3.565672 \\ \mathrm{H} & 4.459664 & -2.139863 & 3.853112 \\ \mathrm{H} & 4.369427 & -0.449315 & 3.319768\end{array}$

Transition State from PC-I to R-I

04

$\begin{array}{lrrr}\text { N } & -0.336737 & 1.089292 & -1.372406 \\ \mathrm{C} & -6.282375 & 0.092394 & -1.127643 \\ \mathrm{O} & -6.745613 & 0.279307 & -2.253627 \\ \mathrm{C} & -4.842179 & 0.499024 & -0.761109 \\ \mathrm{C} & -0.082569 & 3.554161 & -0.962505 \\ \mathrm{C} & -6.468963 & -0.681512 & 1.242359 \\ \mathrm{C} & -7.359104 & -1.814134 & 1.772270 \\ \mathrm{C} & -8.713984 & -1.519201 & 1.103141 \\ \mathrm{C} & -8.342418 & -1.002519 & -0.299341 \\ \mathrm{C} & -4.972452 & -0.968396 & 1.261612 \\ \mathrm{C} & -3.955735 & 0.531748 & -2.027225 \\ \mathrm{C} & -2.587327 & 1.072905 & -1.766223 \\ \mathrm{C} & -1.385240 & 0.321217 & -1.678930 \\ \mathrm{C} & -0.799405 & 2.403951 & -1.254509 \\ \mathrm{C} & -2.203731 & 2.430086 & -1.495880 \\ \mathrm{C} & -2.892044 & 3.652359 & -1.442625 \\ \mathrm{C} & -2.172220 & 4.816843 & -1.146873 \\ \mathrm{C} & -0.792856 & 4.768888 & -0.909551 \\ \mathrm{~N} & -4.282207 & -0.420394 & 0.230042 \\ \mathrm{~N} & -6.981989 & -0.467629 & -0.119460 \\ \mathrm{O} & -4.449819 & -1.629255 & 2.158155 \\ \mathrm{H} & 0.872119 & 0.709942 & -1.255216 \\ \mathrm{H} & -4.885276 & 1.517918 & -0.344813 \\ \mathrm{H} & 0.987308 & 3.520863 & -0.781662 \\ \mathrm{H} & -6.626873 & 0.226673 & 1.847160 \\ \mathrm{H} & -7.405227 & -1.822615 & 2.863099 \\ \mathrm{H} & -6.959374 & -2.778732 & 1.441175 \\ \mathrm{H} & -9.245742 & -0.740119 & 1.660843 \\ \mathrm{H} & -9.362570 & -2.397741 & 1.056513 \\ \mathrm{H} & -9.016501 & -0.222449 & -0.665465 \\ \mathrm{H} & -8.319935 & -1.803041 & -1.047816 \\ \mathrm{H} & -4.487480 & 1.144466 & -2.762309 \\ \mathrm{H} & -3.893390 & -0.481860 & -2.437434 \\ \mathrm{H} & -1.269344 & -0.745838 & -1.826849 \\ \mathrm{H} & -3.960393 & 3.703908 & -1.633859 \\ \mathrm{H} & -2.690469 & 5.769954 & -1.103656 \\ \mathrm{H} & -0.256411 & 5.685549 & -0.683219 \\ \mathrm{H} & -3.272538 & -0.522202 & 0.265552\end{array}$

$\begin{array}{lrrr}\text { Fe } & 2.746783 & -0.411197 & 0.079566 \\ \mathrm{~N} & 3.311397 & -1.980796 & -1.068114 \\ \mathrm{~N} & 1.020824 & -1.372572 & 0.515138 \\ \mathrm{~N} & 4.530733 & 0.476025 & -0.247502 \\ \mathrm{~N} & 2.262572 & 1.055009 & 1.368535 \\ \mathrm{C} & 4.491209 & -2.107276 & -1.754011 \\ \mathrm{C} & 0.043576 & -0.951774 & 1.379418 \\ \mathrm{C} & 2.560501 & -3.083438 & -1.387508 \\ \mathrm{C} & 0.578916 & -2.555962 & -0.019020 \\ \mathrm{C} & 5.539705 & 0.013102 & -1.054752 \\ \mathrm{C} & 1.111322 & 1.150490 & 2.107861 \\ \mathrm{C} & 4.935887 & 1.702947 & 0.209228 \\ \mathrm{C} & 2.969813 & 2.208445 & 1.606692 \\ \mathrm{C} & 4.492469 & -3.333171 & -2.517269 \\ \mathrm{C} & -1.053157 & -1.891657 & 1.389423 \\ \mathrm{C} & 3.293319 & -3.937343 & -2.292364 \\ \mathrm{C} & -0.720873 & -2.884112 & 0.513567 \\ \mathrm{C} & 6.618263 & 0.972796 & -1.097790 \\ \mathrm{C} & 1.098300 & 2.389771 & 2.847049 \\ \mathrm{C} & 6.242525 & 2.022718 & -0.317129 \\ \mathrm{C} & 2.247950 & 3.048947 & 2.530977 \\ \mathrm{H} & 5.311459 & -3.666985 & -3.141359 \\ \mathrm{H} & -1.956187 & -1.794180 & 1.980779 \\ \mathrm{H} & 2.922150 & -4.873026 & -2.690471 \\ \mathrm{H} & -1.286449 & -3.769528 & 0.252470 \\ \mathrm{H} & 7.533094 & 0.842815 & -1.661600 \\ \mathrm{H} & 0.301703 & 2.700226 & 3.510936 \\ \mathrm{H} & 6.785138 & 2.934567 & -0.102879 \\ \mathrm{H} & 2.594024 & 4.011762 & 2.884550 \\ \mathrm{C} & 5.533003 & -1.187431 & -1.750670 \\ \mathrm{C} & 0.080563 & 0.217669 & 2.128705 \\ \mathrm{C} & 4.208854 & 2.523293 & 1.064324 \\ \mathrm{C} & 1.284006 & -3.354262 & -0.912739 \\ \mathrm{H} & 6.405996 & -1.420640 & -2.351815 \\ \mathrm{H} & -0.769660 & 0.427322 & 2.769784 \\ \mathrm{H} & 4.651597 & 3.473493 & 1.345097 \\ \mathrm{H} & 0.807875 & -4.269531 & -1.249246 \\ \mathrm{O} & 2.038638 & 0.402233 & -1.241610 \\ \mathrm{~S} & 3.789555 & -1.664610 & 1.855711 \\ \mathrm{C} & 4.483422 & -0.501372 & 3.087377 \\ \mathrm{H} & 3.702724 & 0.114871 & 3.540846 \\ \mathrm{H} & 4.965857 & -1.095724 & 3.869873 \\ \mathrm{H} & 5.233899 & 0.152763 & 2.633894 \\ & & & \end{array}$

Compound R-I

02

$\begin{array}{lrrr}\text { N } & 3.546705 & -1.709327 & -1.054497 \\ \mathrm{C} & -1.770616 & 1.170224 & -0.535921 \\ \mathrm{O} & -1.700518 & 2.182332 & -1.235014 \\ \mathrm{C} & -0.524194 & 0.322154 & -0.214274 \\ \mathrm{C} & 5.042110 & -0.504590 & 0.559450 \\ \mathrm{C} & -3.045978 & -0.458726 & 0.870851 \\ \mathrm{C} & -4.515304 & -0.861219 & 0.688192 \\ \mathrm{C} & -5.219236 & 0.498570 & 0.529315 \\ \mathrm{C} & -4.222062 & 1.354406 & -0.274464\end{array}$




$\begin{array}{lrrr}\mathrm{C} & -2.029734 & -1.541771 & 0.534706 \\ \mathrm{C} & 0.514329 & 0.468437 & -1.351653 \\ \mathrm{C} & 1.834196 & -0.149151 & -1.031519 \\ \mathrm{C} & 2.356200 & -1.393326 & -1.534595 \\ \mathrm{C} & 3.884711 & -0.648683 & -0.182242 \\ \mathrm{C} & 2.854690 & 0.336174 & -0.141039 \\ \mathrm{C} & 3.000655 & 1.478120 & 0.651952 \\ \mathrm{C} & 4.177595 & 1.623339 & 1.405494 \\ \mathrm{C} & 5.178032 & 0.650570 & 1.361276 \\ \mathrm{~N} & -0.880263 & -1.084387 & -0.027777 \\ \mathrm{~N} & -2.920240 & 0.726107 & 0.009228 \\ \mathrm{O} & -2.238901 & -2.726276 & 0.779449 \\ \mathrm{H} & -0.084251 & 0.726633 & 0.710841 \\ \mathrm{H} & 5.824291 & -1.257066 & 0.524336 \\ \mathrm{H} & -2.869745 & -0.174490 & 1.921348 \\ \mathrm{H} & -4.887035 & -1.448373 & 1.530187 \\ \mathrm{H} & -4.618304 & -1.465007 & -0.219947 \\ \mathrm{H} & -5.392435 & 0.948604 & 1.513245 \\ \mathrm{H} & -6.185938 & 0.417420 & 0.025792 \\ \mathrm{H} & -4.214950 & 2.406303 & 0.026361 \\ \mathrm{H} & -4.411368 & 1.317254 & -1.353308 \\ \mathrm{H} & 0.621839 & 1.542913 & -1.532267 \\ \mathrm{H} & 0.096066 & 0.025252 & -2.262020 \\ \mathrm{H} & 1.850526 & -2.035814 & -2.250407 \\ \mathrm{H} & 2.231602 & 2.245346 & 0.686934 \\ \mathrm{H} & 4.310226 & 2.503663 & 2.027218 \\ \mathrm{H} & 6.080352 & 0.783244 & 1.951261 \\ \mathrm{H} & -0.151292 & -1.778242 & -0.156905 \\ & & & \end{array}$

Compound PC-II

04

$\begin{array}{rrrr}N & -1.081610 & 3.574209 & -0.367355 \\ C & -4.828508 & -1.149960 & -0.914314 \\ \text { O } & -5.533899 & -0.916569 & -1.898068 \\ C & -3.788991 & -0.146194 & -0.375655 \\ C & -2.503315 & 5.301087 & 0.802224 \\ C & -4.090212 & -2.589002 & 0.981454 \\ C & -4.153208 & -4.119975 & 1.071138 \\ C & -5.565358 & -4.437310 & 0.546092 \\ C & -5.790792 & -3.409935 & -0.578320 \\ C & -2.682751 & -2.007974 & 0.859103 \\ C & -3.383093 & 0.869615 & -1.460637 \\ C & -2.691026 & 2.087988 & -0.920902 \\ C & -1.346592 & 2.367578 & -0.983335 \\ C & -2.258739 & 4.106340 & 0.114688 \\ C & -3.301821 & 3.194624 & -0.220817 \\ C & -4.622831 & 3.512460 & 0.146590 \\ C & -4.869562 & 4.698582 & 0.829846 \\ C & -3.819262 & 5.582916 & 1.156644 \\ N & -2.616331 & -0.858230 & 0.145078 \\ N & -4.914524 & -2.291578 & -0.199439 \\ O & -1.716390 & -2.557472 & 1.386847 \\ H & -0.160371 & 3.978104 & -0.266311 \\ H & -4.278730 & 0.406373 & 0.442936 \\ H & -1.694789 & 5.983956 & 1.049285\end{array}$

\begin{tabular}{|c|c|c|c|}
\hline & -4.549691 & -2.143610 & \\
\hline & -3.97 & -4.479351 & \\
\hline & -3.385861 & -4.554511 & \\
\hline & -6.304170 & -4.289377 & $4 \angle U I$ \\
\hline & -5.662989 & -5.465548 & 3772 \\
\hline & -6.8 & -3.0 & 85 \\
\hline & 545 & & \\
\hline & -4.3 & & \\
\hline & & & \\
\hline & & & \\
\hline & & & \\
\hline & & & \\
\hline & & & \\
\hline & 61 & & \\
\hline & & & \\
\hline & & & \\
\hline & & & \\
\hline & & & \\
\hline & & & \\
\hline & & & \\
\hline & & & \\
\hline & & & \\
\hline & & & \\
\hline & & & \\
\hline & 2.0 & & \\
\hline & & & \\
\hline & & & \\
\hline & 2.54 & & -4.2 \\
\hline & & & \\
\hline & 2.488075 & -1.0 & -4.2 \\
\hline & 2.07 & & -0.7 \\
\hline & 2.50 & & \\
\hline & & & \\
\hline & & & \\
\hline & & & \\
\hline & & & \\
\hline & 643 & & \\
\hline & 52 & & -5 \\
\hline & 77 & & \\
\hline & 24 & & -1 \\
\hline & 34 & -1 & \\
\hline & & & \\
\hline & & & \\
\hline & & & -2 \\
\hline & & -2.7 & \\
\hline & & & \\
\hline & & & -2 \\
\hline & & & -3.2 \\
\hline & & & \\
\hline & 2.26 & & \\
\hline & & & -3.2 \\
\hline & & -0 & -0.1 \\
\hline & & -0.6 & \\
\hline & & & \\
\hline & $+.9<500$ & - & 1.044 \\
\hline
\end{tabular}



$\mathrm{H}$
6.455336
0.788974
1.094717
H 5.251367
1.729051
0.154304

Transition State from PC-II to R-II

04

\begin{tabular}{|c|c|c|c|}
\hline & 159 & & \\
\hline & 7482 & & \\
\hline & & & \\
\hline & 6211 & 1773 & $-c$ \\
\hline & 106 & 3346 & \\
\hline & -3.494523 & 34155 & $-c$ \\
\hline & -4.238724 & -3.750085 & -1.27773 \\
\hline & 577 & 5115 & 6387 \\
\hline & 164 & 30908 & -0 \\
\hline & -2.152716 & -2.239268 & -0 \\
\hline & -2.95 & 1.004022 & \\
\hline & $-2.0<$ & & \\
\hline & -0.8 & & \\
\hline & -1.1 & 012 & \\
\hline & -2.2 & 3693 & \\
\hline & -3.27 & 80 & \\
\hline & & & \\
\hline & -2.0 & & \\
\hline & & & \\
\hline & -4.5 & -1 & \\
\hline & -1 & & \\
\hline & & & \\
\hline & -2 & & \\
\hline & -0.2 & & \\
\hline & -3.2 & & \\
\hline & -3 & & \\
\hline & -4.2 & & \\
\hline & & & \\
\hline & & & \\
\hline & -6.3 & & \\
\hline & & & \\
\hline & & & \\
\hline & -2.7 & & \\
\hline & -0.3 & & \\
\hline & -4 & & \\
\hline & -3.9 & & \\
\hline & -2.0 & & \\
\hline & -0.4 & -0. & -0 . \\
\hline & & & \\
\hline & 3.08 & 2543 & 39664 \\
\hline & & 601 & 031 \\
\hline & 2.65 & 836 & 1045 \\
\hline & 0.61 & 0.473256 & 62763 \\
\hline & 4.02 & 1111 & 13016 \\
\hline & 0.10 & 8977 & 1. \\
\hline & 3.16 & -2.802187 & -1.20089 \\
\hline & & 5289 & \\
\hline & & 1.473291 & -1.173867 \\
\hline & -0.3 & -0.097371 & \\
\hline & .2 & & 0.1 \\
\hline
\end{tabular}

$\begin{array}{rrrr}\text { C } & 0.524598 & 1.829397 & 1.761219 \\ \mathrm{C} & 4.703983 & -1.899973 & -2.573309 \\ \mathrm{C} & -0.138916 & -3.870008 & 1.972559 \\ \mathrm{C} & 4.172889 & -3.094155 & -2.192417 \\ \mathrm{C} & 0.699559 & -4.518991 & 1.119103 \\ \mathrm{C} & 3.938226 & 2.880021 & -1.305458 \\ \mathrm{C} & -1.020969 & 0.923493 & 3.124164 \\ \mathrm{C} & 3.093490 & 3.532406 & -0.456448 \\ \mathrm{C} & -0.497003 & 2.119823 & 2.737185 \\ \mathrm{H} & 5.486387 & -1.706462 & -3.295649 \\ \mathrm{H} & -0.864265 & -4.288712 & 2.658107 \\ \mathrm{H} & 4.426972 & -4.088633 & -2.535690 \\ \mathrm{H} & 0.805301 & -5.583049 & 0.952783 \\ \mathrm{H} & 4.687823 & 3.296686 & -1.965755 \\ \mathrm{H} & -1.807527 & 0.730688 & 3.842110 \\ \mathrm{H} & 3.009192 & 4.595590 & -0.271192 \\ \mathrm{H} & -0.767514 & 3.114880 & 3.065235 \\ \mathrm{C} & 4.293761 & 0.475648 & -1.902035 \\ \mathrm{C} & -0.565822 & -1.460008 & 2.497664 \\ \mathrm{C} & 1.300922 & 2.789314 & 1.125432 \\ \mathrm{C} & 2.416156 & -3.767794 & -0.536455 \\ \mathrm{H} & 5.066640 & 0.784943 & -2.598322 \\ \mathrm{H} & -1.335297 & -1.770807 & 3.196852 \\ \mathrm{H} & 1.114446 & 3.828106 & 1.375762 \\ \mathrm{H} & 2.597631 & -4.806384 & -0.792699 \\ \mathrm{O} & 0.619764 & -0.338817 & -1.011237 \\ \mathrm{~S} & 3.387000 & -0.787264 & 2.002265 \\ \mathrm{C} & 3.843603 & 0.832576 & 2.717955 \\ \mathrm{H} & 2.966448 & 1.358701 & 3.104442 \\ \mathrm{H} & 4.524823 & 0.632626 & 3.551211 \\ \mathrm{H} & 4.351737 & 1.467818 & 1.988249\end{array}$

Compound R-II

02

$\begin{array}{rrrr}N & -3.068676 & -1.986608 & 0.268667 \\ C & 1.838962 & 1.398401 & -0.301609 \\ \mathrm{O} & 1.665946 & 2.577640 & -0.004289 \\ \mathrm{C} & 0.607305 & 0.512059 & -0.646042 \\ \mathrm{C} & -5.137800 & -0.593746 & -0.114142 \\ \mathrm{C} & 3.302596 & -0.630996 & -0.568764 \\ \mathrm{C} & 4.365301 & -1.002555 & 0.470677 \\ \mathrm{C} & 5.224630 & 0.276227 & 0.523773 \\ \mathrm{C} & 4.222516 & 1.436392 & 0.328523 \\ \mathrm{C} & 2.003218 & -1.437622 & -0.538494 \\ \mathrm{C} & -0.245983 & 0.385108 & 0.668259 \\ \mathrm{C} & -1.548222 & -0.334046 & 0.482195 \\ \mathrm{C} & -1.738989 & -1.696167 & 0.487635 \\ \mathrm{C} & -3.774508 & -0.811637 & 0.116757 \\ \mathrm{C} & -2.841906 & 0.258006 & 0.241774 \\ \mathrm{C} & -3.309897 & 1.580292 & 0.126310 \\ \mathrm{C} & -4.662564 & 1.800740 & -0.104274 \\ \mathrm{C} & -5.567869 & 0.724173 & -0.223784 \\ \mathrm{~N} & 0.969604 & -0.774322 & -1.162743 \\ \mathrm{~N} & 3.044276 & 0.790955 & -0.282924 \\ \mathrm{O} & 1.931957 & -2.580584 & -0.110472\end{array}$




$\begin{array}{rrrr}\mathrm{H} & -3.461429 & -2.915562 & 0.235496 \\ \mathrm{H} & 0.012047 & 1.046565 & -1.392574 \\ \mathrm{H} & -5.833050 & -1.423954 & -0.204905 \\ \mathrm{H} & 3.722580 & -0.730006 & -1.581282 \\ \mathrm{H} & 4.930613 & -1.891192 & 0.182063 \\ \mathrm{H} & 3.883172 & -1.198629 & 1.434275 \\ \mathrm{H} & 5.955036 & 0.268919 & -0.292160 \\ \mathrm{H} & 5.776547 & 0.371921 & 1.462192 \\ \mathrm{H} & 4.610832 & 2.226962 & -0.321113 \\ \mathrm{H} & 3.916338 & 1.898715 & 1.272026 \\ \mathrm{H} & -0.411680 & 1.410421 & 1.013361 \\ \mathrm{H} & 0.369993 & -0.115847 & 1.424901 \\ \mathrm{H} & -1.014399 & -2.489144 & 0.615845 \\ \mathrm{H} & -2.624391 & 2.419496 & 0.215250 \\ \mathrm{H} & -5.032882 & 2.818356 & -0.195049 \\ \mathrm{H} & -6.619731 & 0.928568 & -0.403999\end{array}$

Transition State from $\mathbf{R}$-II to $\mathbf{R}$-III

02

$\begin{array}{lrrr}\mathrm{N} & -2.338248 & -1.782052 & 0.475069 \\ \mathrm{C} & 1.933448 & 1.512669 & -0.181575 \\ \mathrm{O} & 2.058894 & 2.702385 & 0.125154 \\ \mathrm{C} & 0.537341 & 0.908657 & -0.369460 \\ \mathrm{C} & -4.622182 & -1.106776 & -0.355875 \\ \mathrm{C} & 2.890735 & -0.756604 & -0.645921 \\ \mathrm{C} & 4.074481 & -1.346518 & 0.129538 \\ \mathrm{C} & 5.143821 & -0.246858 & -0.009728 \\ \mathrm{C} & 4.348934 & 1.074951 & 0.039727 \\ \mathrm{C} & 1.509286 & -1.360924 & -0.325239 \\ \mathrm{C} & -0.365050 & 1.277650 & 0.852540 \\ \mathrm{C} & -1.488169 & 0.289379 & 0.821722 \\ \mathrm{C} & -1.219449 & -1.105681 & 0.872291 \\ \mathrm{C} & -3.334571 & -0.883138 & 0.124791 \\ \mathrm{C} & -2.815449 & 0.431926 & 0.331866 \\ \mathrm{C} & -3.627429 & 1.545450 & 0.025327 \\ \mathrm{C} & -4.912735 & 1.325481 & -0.448249 \\ \mathrm{C} & -5.404278 & 0.015046 & -0.634552 \\ \mathrm{~N} & 0.470139 & -0.538664 & -0.518892 \\ \mathrm{~N} & 2.979812 & 0.675706 & -0.327892 \\ \mathrm{O} & 1.433628 & -2.562620 & -0.030201 \\ \mathrm{H} & -2.427303 & -2.787993 & 0.464407 \\ \mathrm{H} & 0.099071 & 1.383704 & -1.258702 \\ \mathrm{H} & -5.002807 & -2.111645 & -0.511650 \\ \mathrm{H} & 3.052683 & -0.903312 & -1.725400 \\ \mathrm{H} & 4.389042 & -2.311920 & -0.272224 \\ \mathrm{H} & 3.790374 & -1.490144 & 1.178012 \\ \mathrm{H} & 5.654658 & -0.342403 & -0.974357 \\ \mathrm{H} & 5.904913 & -0.293345 & 0.773848 \\ \mathrm{H} & 4.734002 & 1.830673 & -0.652713 \\ \mathrm{H} & 4.331330 & 1.522036 & 1.039683 \\ \mathrm{H} & -0.705282 & 2.312834 & 0.785343 \\ \mathrm{H} & 0.214037 & 1.178042 & 1.778839 \\ \mathrm{H} & -0.428730 & -1.638618 & 1.377713 \\ \mathrm{H} & -3.252609 & 2.555097 & 0.166886 \\ \mathrm{H} & -5.553270 & 2.170459 & -0.682559\end{array}$

H $\quad-6.414612 \quad-0.124762 \quad-1.007942$

Compound R-III

02

$\begin{array}{llll}N & -1.825839 & -1.536027 & 0.207325\end{array}$

$\begin{array}{llll}\text { C } & 1.975028 & 1.532017 & -0.131974\end{array}$

$\begin{array}{llll}\text { O } & 2.210570 & 2.711006 & 0.136743\end{array}$

$\begin{array}{llll}\text { C } & 0.547036 & 1.018466 & -0.335224\end{array}$

$\begin{array}{llll}\text { C } & -4.258316 & -1.392632 & -0.397436\end{array}$

$\begin{array}{llll}\text { C } & 2.670484 & -0.827879 & -0.577819\end{array}$

C $3.948188-1.524530-0.091778$

C $5.039840-0.475963-0.371066$

$\begin{array}{llll}\text { C } & 4.361042 & 0.868827 & -0.056935\end{array}$

$\begin{array}{llll}\text { C } & 1.381177 & -1.311158 & 0.085301\end{array}$

$\begin{array}{llll}\text { C } & -0.528403 & 1.796064 & 0.475666\end{array}$

$\begin{array}{llll}\text { C } & -1.547686 & 0.728873 & 0.721137\end{array}$

$\begin{array}{llll}\text { C } & -0.874169 & -0.617174 & 0.825944\end{array}$

$\begin{array}{llll}\text { C } & -3.038255 & -0.875192 & 0.024054\end{array}$

$\begin{array}{llll}\text { C } & -2.866484 & 0.530932 & 0.258928\end{array}$

C $\quad-3.938795 \quad 1.410550 \quad-0.002590$

C $\quad-5.155799 \quad 0.886643 \quad-0.430077$

$\begin{array}{llll}C & -5.317060 & -0.498095 & -0.613139\end{array}$

$\begin{array}{llll}\mathrm{N} & 0.395058 & -0.382956 & 0.116466\end{array}$

$\begin{array}{llll}\mathrm{N} & 2.933692 & 0.593060 & -0.285417\end{array}$

$\begin{array}{llll}\mathrm{O} & 1.249026 & -2.450859 & 0.530648\end{array}$

H $\quad-1.823790 \quad-2.498270 \quad 0.521265$

H $\quad 0.312492 \quad 1.089499-1.407576$

H $\quad-4.383988-2.457226 \quad-0.573881$

H $\quad 2.555439-0.969950-1.664638$

H $4.117559-2.473966 \quad-0.604246$

$\begin{array}{llll}H & 3.865125 & -1.729827 & 0.980713\end{array}$

H $\quad 5.333211-0.510276-1.426391$

H $\quad 5.939257 \quad-0.631425 \quad 0.230411$

H $4.700409 \quad 1.686883 \quad-0.699142$

H $4.510663 \quad 1.177494 \quad 0.984630$

H $\quad-0.915425 \quad 2.650057 \quad-0.084502$

H $\quad-0.074420 \quad 2.180189 \quad 1.398402$

H $\quad-0.631596 \quad-0.957224 \quad 1.843486$

$\begin{array}{llll}\mathrm{H} & -3.817520 & 2.479030 & 0.153321\end{array}$

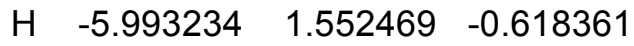

H $\quad-6.278311 \quad-0.885672 \quad-0.939195$

3-methylindole

01

$\begin{array}{lrrr}\text { N } & 0.632687 & 1.814318 & 0.000440 \\ \mathrm{C} & -1.790518 & 1.108332 & 0.000038 \\ \mathrm{C} & 2.641172 & -1.314436 & 0.000037 \\ \mathrm{C} & 1.608295 & -0.226725 & -0.000164 \\ \mathrm{C} & 1.834240 & 1.127603 & -0.000085 \\ \mathrm{C} & -0.405047 & 0.907751 & 0.000049 \\ \mathrm{C} & 0.174859 & -0.393770 & -0.000045 \\ \mathrm{C} & -0.675624 & -1.514387 & 0.000006 \\ \mathrm{C} & -2.052337 & -1.319799 & -0.000023 \\ \mathrm{C} & -2.603782 & -0.020530 & -0.000047 \\ \mathrm{H} & 0.536398 & 2.818369 & -0.001853\end{array}$




$\begin{array}{rrrr}\mathrm{H} & -2.214783 & 2.108908 & 0.000084 \\ \mathrm{H} & 2.550605 & -1.962805 & -0.881613 \\ \mathrm{H} & 3.653333 & -0.896375 & 0.000588 \\ \mathrm{H} & 2.771096 & 1.668609 & -0.000155 \\ \mathrm{H} & -0.261497 & -2.519740 & 0.000054 \\ \mathrm{H} & -2.717631 & -2.179206 & -0.000028 \\ \mathrm{H} & -3.683712 & 0.100979 & -0.000093 \\ \mathrm{H} & 2.549839 & -1.963186 & 0.881338\end{array}$

Transition State from R-III to R-IV

02

$\begin{array}{lrrr}\mathrm{N} & -5.566010 & 0.067828 & -0.750328 \\ \mathrm{C} & -3.454605 & 0.700912 & -1.990373 \\ \mathrm{C} & -4.832501 & -3.102094 & 1.047864 \\ \mathrm{C} & -4.862720 & -1.840573 & 0.238460 \\ \mathrm{C} & -5.927209 & -0.991043 & 0.067570 \\ \mathrm{C} & -4.252694 & -0.077585 & -1.125277 \\ \mathrm{C} & -3.766090 & -1.271044 & -0.515001 \\ \mathrm{C} & -2.448732 & -1.672261 & -0.752188 \\ \mathrm{C} & -1.566897 & -0.813989 & -1.490162 \\ \mathrm{C} & -2.154795 & 0.306253 & -2.202178 \\ \mathrm{H} & -6.176279 & 0.822785 & -1.025463 \\ \mathrm{H} & -3.867343 & 1.568247 & -2.499427 \\ \mathrm{H} & -4.064844 & -3.062957 & 1.832136 \\ \mathrm{H} & -5.796425 & -3.281852 & 1.535252 \\ \mathrm{H} & -6.929326 & -1.051219 & 0.470160 \\ \mathrm{H} & -2.068121 & -2.601321 & -0.336045 \\ \mathrm{H} & -0.719156 & -1.296211 & -1.977442 \\ \mathrm{H} & -1.526915 & 0.877519 & -2.879386 \\ \mathrm{H} & -4.605915 & -3.979222 & 0.427063 \\ \mathrm{~N} & 0.370239 & 2.191987 & -0.782233 \\ \mathrm{C} & 3.033387 & -1.588206 & 0.786701 \\ \mathrm{O} & 2.865040 & -2.775631 & 1.068131 \\ \mathrm{C} & 1.886553 & -0.574303 & 0.794455 \\ \mathrm{C} & -0.928421 & 3.435362 & 0.975946 \\ \mathrm{C} & 4.450511 & 0.349733 & 0.084470 \\ \mathrm{C} & 5.747855 & 0.295457 & -0.733970 \\ \mathrm{C} & 6.529909 & -0.847469 & -0.062876 \\ \mathrm{C} & 5.448422 & -1.877127 & 0.310084 \\ \mathrm{C} & 3.250176 & 0.937567 & -0.657262 \\ \mathrm{C} & 0.495758 & -1.170169 & 0.492859 \\ \mathrm{C} & -0.249851 & -0.002293 & -0.133561 \\ \mathrm{C} & 0.783946 & 0.807292 & -0.931227 \\ \mathrm{C} & -0.479647 & 2.290720 & 0.321110 \\ \mathrm{C} & -0.896340 & 0.998166 & 0.725834 \\ \mathrm{C} & -1.777322 & 0.862332 & 1.800922 \\ \mathrm{C} & -2.236110 & 2.008060 & 2.462871 \\ \mathrm{C} & -1.813846 & 3.277280 & 2.051555 \\ \mathrm{~N} & 2.057237 & 0.441918 & -0.260771 \\ \mathrm{~N} & 4.233375 & -1.060095 & 0.456805 \\ \mathrm{O} & 3.369234 & 1.804301 & -1.526856 \\ \mathrm{H} & 1.075567 & 2.898458 & -0.953090 \\ \mathrm{H} & 1.873819 & -0.086820 & 1.781145 \\ \mathrm{H} & -0.594050 & 4.422834 & 0.669273 \\ \mathrm{H} & 4.605929 & 0.956180 & 0.991746\end{array}$

$\begin{array}{rrrr}\mathrm{H} & 6.278617 & 1.249778 & -0.724339 \\ \mathrm{H} & 5.511617 & 0.050567 & -1.775019 \\ \mathrm{H} & 7.026897 & -0.481508 & 0.842661 \\ \mathrm{H} & 7.296375 & -1.275343 & -0.714376 \\ \mathrm{H} & 5.663680 & -2.414495 & 1.238490 \\ \mathrm{H} & 5.293505 & -2.623200 & -0.478554 \\ \mathrm{H} & 0.015751 & -1.549716 & 1.396973 \\ \mathrm{H} & 0.606425 & -2.008434 & -0.205451 \\ \mathrm{H} & 0.881116 & 0.552887 & -1.991896 \\ \mathrm{H} & -2.101273 & -0.123659 & 2.122621 \\ \mathrm{H} & -2.918971 & 1.910454 & 3.302204 \\ \mathrm{H} & -2.171186 & 4.159557 & 2.576477\end{array}$

\section{Compound R-IV}

02

$\begin{array}{rrrr}\text { N } & -5.707588 & -0.106556 & -0.984684 \\ \mathrm{C} & -3.514493 & 0.747008 & -1.934977 \\ \mathrm{C} & -4.888469 & -3.070003 & 1.101239 \\ \mathrm{C} & -4.944779 & -1.862661 & 0.215120 \\ \mathrm{C} & -6.064454 & -1.160100 & -0.159222 \\ \mathrm{C} & -4.344925 & -0.109478 & -1.157341 \\ \mathrm{C} & -3.817546 & -1.201966 & -0.414240 \\ \mathrm{C} & -2.448342 & -1.453522 & -0.410841 \\ \mathrm{C} & -1.486476 & -0.564979 & -1.162549 \\ \mathrm{C} & -2.173712 & 0.531195 & -1.950510 \\ \mathrm{H} & -6.350730 & 0.555945 & -1.391436 \\ \mathrm{H} & -3.952497 & 1.553633 & -2.518845 \\ \mathrm{H} & -4.265773 & -2.894119 & 1.988841 \\ \mathrm{H} & -5.887756 & -3.353622 & 1.447858 \\ \mathrm{H} & -7.101804 & -1.324991 & 0.098605 \\ \mathrm{H} & -2.045811 & -2.311287 & 0.120373 \\ \mathrm{H} & -0.918361 & -1.191505 & -1.880610 \\ \mathrm{H} & -1.538564 & 1.179253 & -2.547167 \\ \mathrm{H} & -4.458452 & -3.936931 & 0.581580 \\ \mathrm{~N} & 0.440190 & 2.192594 & -0.853279 \\ \mathrm{C} & 2.945619 & -1.604629 & 0.806795 \\ \mathrm{O} & 2.746688 & -2.765072 & 1.168401 \\ \mathrm{C} & 1.826344 & -0.562040 & 0.744898 \\ \mathrm{C} & -0.594181 & 3.503371 & 1.028183 \\ \mathrm{C} & 4.411405 & 0.243809 & -0.024422 \\ \mathrm{C} & 5.697867 & 0.098899 & -0.849304 \\ \mathrm{C} & 6.454800 & -1.018436 & -0.109762 \\ \mathrm{C} & 5.349277 & -1.989588 & 0.342068 \\ \mathrm{C} & 3.223802 & 0.825641 & -0.789041 \\ \mathrm{C} & 0.434089 & -1.139490 & 0.474653 \\ \mathrm{C} & -0.340452 & 0.004862 & -0.222402 \\ \mathrm{C} & 0.758799 & 0.783485 & -1.023731 \\ \mathrm{C} & -0.305836 & 2.334251 & 0.323546 \\ \mathrm{C} & -0.819854 & 1.090218 & 0.732157 \\ \mathrm{C} & -1.636557 & 1.010413 & 1.853518 \\ \mathrm{C} & -1.936942 & 2.178235 & 2.571118 \\ \mathrm{C} & -1.416667 & 3.407428 & 2.157253 \\ \mathrm{~N} & 2.019981 & 0.369075 & -0.376936 \\ \mathrm{~N} & 4.157585 & -1.131678 & 0.440048 \\ \mathrm{O} & 3.362002 & 1.657868 & -1.688826\end{array}$




$\begin{array}{rrrr}\mathrm{H} & 1.204444 & 2.833371 & -1.036675 \\ \mathrm{H} & 1.828609 & -0.007655 & 1.695942 \\ \mathrm{H} & -0.189497 & 4.460917 & 0.711799 \\ \mathrm{H} & 4.595711 & 0.901995 & 0.840492 \\ \mathrm{H} & 6.254623 & 1.036435 & -0.908040 \\ \mathrm{H} & 5.443984 & -0.208518 & -1.869469 \\ \mathrm{H} & 6.972483 & -0.606876 & 0.764048 \\ \mathrm{H} & 7.201326 & -1.510890 & -0.738279 \\ \mathrm{H} & 5.558649 & -2.466591 & 1.304176 \\ \mathrm{H} & 5.166973 & -2.784081 & -0.391378 \\ \mathrm{H} & -0.049433 & -1.474747 & 1.393645 \\ \mathrm{H} & 0.523382 & -2.003791 & -0.193005 \\ \mathrm{H} & 0.816040 & 0.530400 & -2.085830 \\ \mathrm{H} & -2.038898 & 0.053708 & 2.175041 \\ \mathrm{H} & -2.571343 & 2.124434 & 3.451162 \\ \mathrm{H} & -1.647894 & 4.307559 & 2.721269\end{array}$

Compound PDT $_{\mathrm{C}-\mathrm{C}}$

01

$\begin{array}{lrrr}\text { N } & -5.659156 & -1.125029 & -1.454404 \\ \mathrm{C} & -3.636937 & 0.287975 & -2.002739 \\ \mathrm{C} & -4.842626 & -2.913365 & 1.701484 \\ \mathrm{C} & -4.913807 & -2.080493 & 0.456239 \\ \mathrm{C} & -5.964187 & -1.998092 & -0.424986 \\ \mathrm{C} & -4.393358 & -0.620818 & -1.254922 \\ \mathrm{C} & -3.891169 & -1.202051 & -0.058515 \\ \mathrm{C} & -2.598926 & -0.858948 & 0.378889 \\ \mathrm{C} & -1.828157 & 0.039517 & -0.358017 \\ \mathrm{C} & -2.367123 & 0.609054 & -1.541098 \\ \mathrm{H} & -4.025603 & 0.731838 & -2.915406 \\ \mathrm{H} & -4.691284 & -2.295760 & 2.596717 \\ \mathrm{H} & -5.765336 & -3.484911 & 1.846327 \\ \mathrm{H} & -6.922195 & -2.500063 & -0.401743 \\ \mathrm{H} & -2.212353 & -1.301169 & 1.292335 \\ \mathrm{H} & -1.770426 & 1.321956 & -2.102336 \\ \mathrm{H} & -4.010942 & -3.629420 & 1.665855 \\ \mathrm{~N} & 0.593831 & 2.105467 & -1.321278 \\ \mathrm{C} & 2.647188 & -1.472023 & 1.206004 \\ \mathrm{O} & 2.320724 & -2.379984 & 1.971345 \\ \mathrm{C} & 1.743132 & -0.267752 & 0.930557 \\ \mathrm{C} & 0.275050 & 4.133009 & 0.138959 \\ \mathrm{C} & 4.222796 & -0.371656 & -0.394160 \\ \mathrm{C} & 5.272186 & -1.061090 & -1.277193 \\ \mathrm{C} & 5.947897 & -2.042169 & -0.302816 \\ \mathrm{C} & 4.797800 & -2.540030 & 0.590544 \\ \mathrm{C} & 3.031205 & 0.210497 & -1.152344 \\ \mathrm{C} & 0.246864 & -0.561871 & 1.054812 \\ \mathrm{C} & -0.437501 & 0.466807 & 0.120186 \\ \mathrm{C} & 0.602250 & 0.676218 & -1.040318 \\ \mathrm{C} & 0.173294 & 2.774577 & -0.163366 \\ \mathrm{C} & -0.467513 & 1.882785 & 0.712514 \\ \mathrm{C} & -1.032749 & 2.345307 & 1.893174 \\ \mathrm{C} & -0.937517 & 3.708236 & 2.211029 \\ \mathrm{C} & -0.287126 & 4.585870 & 1.338651 \\ \mathrm{~N} & 1.870299 & 0.193038 & -0.459589\end{array}$

$\begin{array}{rrrr}\mathrm{N} & 3.823864 & -1.437645 & 0.542030 \\ \mathrm{O} & 3.135572 & 0.674322 & -2.290391 \\ \mathrm{H} & 1.409454 & 2.446032 & -1.819171 \\ \mathrm{H} & 2.032769 & 0.539380 & 1.620800 \\ \mathrm{H} & 0.776938 & 4.820272 & -0.536656 \\ \mathrm{H} & 4.691019 & 0.457025 & 0.161800 \\ \mathrm{H} & 5.965118 & -0.345365 & -1.724354 \\ \mathrm{H} & 4.769942 & -1.598617 & -2.088684 \\ \mathrm{H} & 6.693005 & -1.515607 & 0.304226 \\ \mathrm{H} & 6.455068 & -2.864431 & -0.814194 \\ \mathrm{H} & 5.104382 & -2.735901 & 1.622326 \\ \mathrm{H} & 4.333054 & -3.453703 & 0.201257 \\ \mathrm{H} & -0.094246 & -0.486164 & 2.089249 \\ \mathrm{H} & 0.048146 & -1.579994 & 0.707569 \\ \mathrm{H} & 0.390496 & 0.106782 & -1.947355 \\ \mathrm{H} & -1.542012 & 1.660460 & 2.567340 \\ \mathrm{H} & -1.369184 & 4.080411 & 3.135596 \\ \mathrm{H} & -0.213885 & 5.640391 & 1.591944 \\ \mathrm{H} & -6.269967 & -0.895924 & -2.223785\end{array}$

\section{Compound C-III}

11

N $\quad-1.843818 \quad-1.512494 \quad 0.257149$

C $\quad 2.006729 \quad 1.529724-0.091016$

$\begin{array}{llll}\mathrm{O} & 2.224830 & 2.683849 & 0.272329\end{array}$

$\begin{array}{llll}\text { C } & 0.587546 & 1.062692 & -0.442791\end{array}$

C $\quad-4.287649-1.445226-0.199211$

C $2.679528-0.823946-0.609120$

C $3.924232-1.572061-0.113030$

$\begin{array}{llll}\text { C } & 5.040947 & -0.524621 & -0.271163\end{array}$

$\begin{array}{llll}\text { C } & 4.368761 & 0.804905 & 0.108496\end{array}$

C $1.361142 \quad-1.300584-0.007939$

$\begin{array}{llll}\text { C } & -0.524615 & 1.849467 & 0.315826\end{array}$

$\begin{array}{llll}C & -1.532267 & 0.775006 & 0.511678\end{array}$

C $\quad-0.844548 \quad-0.543404 \quad 0.707564$

C $\quad-3.020865 \quad-0.894400 \quad 0.093849$

$\begin{array}{llll}\text { C } & -2.845435 & 0.556468 & 0.198926\end{array}$

C $\quad-3.945640 \quad 1.432272 \quad-0.082730$

$\begin{array}{llll}\text { C } & -5.152305 & 0.871457 & -0.383810\end{array}$

$\begin{array}{llll}C & -5.313006 & -0.557853 & -0.432423\end{array}$

$\begin{array}{llll}\mathrm{N} & 0.367371 & -0.360285 & -0.057767\end{array}$

$\begin{array}{llll}\mathrm{N} & 2.951094 & 0.577184 & -0.227982\end{array}$

$\begin{array}{llll}\mathrm{O} & 1.171001 & -2.411728 & 0.469505\end{array}$

H $\quad-1.729771 \quad-2.508472 \quad 0.409070$

H $\quad 0.442911 \quad 1.175059-1.524728$

H $\quad-4.435437 \quad-2.517515 \quad-0.257474$

H $\quad 2.595197 \quad-0.906573 \quad-1.704048$

H $4.103346 \quad-2.486239-0.682695$

$\begin{array}{llll}H & 3.789617 & -1.847788 & 0.937837\end{array}$

H $\quad \begin{array}{llll}5.381101 & -0.489612 & -1.311892\end{array}$

$\begin{array}{llll}H & 5.908373 & -0.738371 & 0.357889\end{array}$

H $4.754814 \quad 1.663651 \quad-0.446997$

H $4.456646 \quad 1.026179 \quad 1.178362$

H $\quad-0.898201 \quad 2.702369 \quad-0.250986$

H $\quad-0.125932 \quad 2.219624 \quad 1.268502$ 


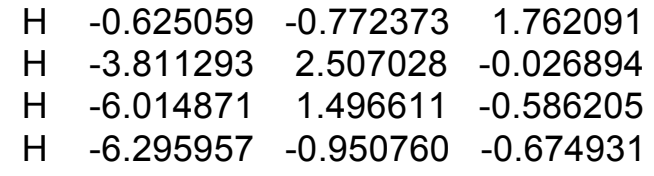

Transition State from $\mathbf{C}$-III to $\mathbf{C}$-IV

11

$\begin{array}{llll}\mathrm{N} & -5.209632 & 0.262283 & -0.586504\end{array}$

C $\quad-3.203395 \quad 0.840413 \quad-1.976389$

$\begin{array}{llll}\text { C } & -4.627184 & -3.106813 & 0.887129\end{array}$

$\begin{array}{llll}\text { C } & -4.614109 & -1.782017 & 0.187942\end{array}$

$\begin{array}{llll}\text { C } & -5.597134 & -0.833208 & 0.186381\end{array}$

C $\quad-3.976616 \quad 0.047186 \quad-1.101810$

$\begin{array}{llll}\text { C } & -3.546267 & -1.243534 & -0.632385\end{array}$

C $\quad-2.301151 \quad-1.707898-1.016250$

$\begin{array}{llll}\text { C } & -1.454746 & -0.860671 & -1.786322\end{array}$

$\begin{array}{llll}\text { C } & -1.968702 & 0.359614 & -2.342749\end{array}$

$\begin{array}{llll}\text { H } & -5.777610 & 1.083321 & -0.746843\end{array}$

H $\quad-3.585522 \quad 1.780169-2.362403$

$\begin{array}{llll}\mathrm{H} & -3.799807 & -3.193684 & 1.602521\end{array}$

$\begin{array}{llll}\mathrm{H} & -5.561637 & -3.249195 & 1.437454\end{array}$

H $\quad-6.561623 \quad-0.834086 \quad 0.674360$

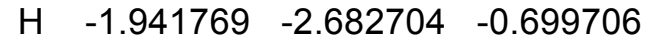

H $\quad-0.577471 \quad-1.302144 \quad-2.248819$

$\begin{array}{llll}\mathrm{H} & -1.352023 & 0.925162 & -3.032984\end{array}$

$\begin{array}{llll}\mathrm{H} & -4.526682 & -3.936026 & 0.176185\end{array}$

$\begin{array}{llll}\mathrm{N} & 0.212167 & 2.155357 & -0.599177\end{array}$

C $3.064318-1.597984 \quad 0.666610$

$\begin{array}{llll}\text { O } & 2.941516 & -2.816807 & 0.780964\end{array}$

$\begin{array}{llll}\text { C } & 1.864309 & -0.652221 & 0.798628\end{array}$

$\begin{array}{llll}\text { C } & -1.371289 & 3.180726 & 1.038707\end{array}$

$\begin{array}{llll}\text { C } & 4.373960 & 0.491170 & 0.248974\end{array}$

$\begin{array}{llll}\text { C } & 5.701164 & 0.624974 & -0.510089\end{array}$

$\begin{array}{llll}\text { C } & 6.525615 & -0.557286 & 0.029508\end{array}$

$\begin{array}{llll}\text { C } & 5.497739 & -1.688511 & 0.199805\end{array}$

$\begin{array}{llll}\text { C } & 3.162285 & 1.087500 & -0.463797\end{array}$

$\begin{array}{llll}\text { C } & 0.515217 & -1.300054 & 0.400845\end{array}$

$\begin{array}{llll}\text { C } & -0.245062 & -0.095669 & -0.107465\end{array}$

$\begin{array}{llll}\text { C } & 0.745076 & 0.829190 & -0.832893\end{array}$

C $\quad-0.741248 \quad 2.118853 \quad 0.373120$

$\begin{array}{llll}\text { C } & -1.049099 & 0.759509 & 0.704185\end{array}$

$\begin{array}{llll}\text { C } & -1.973371 & 0.475660 & 1.734178\end{array}$

C $\quad-2.581684 \quad 1.527568 \quad 2.395690$

$\begin{array}{llll}C & -2.282206 & 2.863342 & 2.037875\end{array}$

$\begin{array}{llll}\mathrm{N} & 1.982266 & 0.503264 & -0.116804\end{array}$

$\begin{array}{llll}\mathrm{N} & 4.232324 & -0.967634 & 0.425187\end{array}$

$\begin{array}{llll}\mathrm{O} & 3.227386 & 2.015536 & -1.264898\end{array}$

H $\quad 0.721965 \quad 2.979367 \quad-0.886630$

H $\quad 1.813658 \quad-0.299732 \quad 1.838190$

$\mathrm{H} \quad-1.139534 \quad 4.211872 \quad 0.793522$

$\begin{array}{llll}\mathrm{H} & 4.449869 & 0.981310 & 1.232721\end{array}$

H $\quad 6.171736 \quad 1.595531 \quad-0.340323$

H $\quad 5.519506 \quad 0.519766 \quad-1.584766$

H $\quad 6.963572 \quad-0.299222 \quad 1.000007$

H $\quad 7.340634 \quad-0.842364 \quad-0.640232$

$\begin{array}{rrrr}\mathrm{H} & 5.713278 & -2.349326 & 1.043872 \\ \mathrm{H} & 5.407519 & -2.308657 & -0.699634 \\ \mathrm{H} & 0.031034 & -1.787954 & 1.247248 \\ \mathrm{H} & 0.690544 & -2.048660 & -0.375847 \\ \mathrm{H} & 0.887564 & 0.661779 & -1.902258 \\ \mathrm{H} & -2.193115 & -0.551810 & 2.003906 \\ \mathrm{H} & -3.290257 & 1.334059 & 3.193926 \\ \mathrm{H} & -2.773446 & 3.671477 & 2.572534\end{array}$

Compound C-IV

11

$\begin{array}{llll}\mathrm{N} & -5.647849 & -0.163982 & -1.064375\end{array}$

$\begin{array}{llll}\text { C } & -3.500934 & 0.740552 & -1.956776\end{array}$

$\begin{array}{llll}\text { C } & -4.894590 & -3.074322 & 1.141437\end{array}$

$\begin{array}{llll}\text { C } & -4.942774 & -1.893042 & 0.225743\end{array}$

C $\quad-6.037991-1.219164-0.210976$

C $-4.324256-0.131559-1.192630$

C $\quad-3.798900 \quad-1.219538 \quad-0.384328$

C $\quad-2.456582-1.414697 \quad-0.332673$

C $\quad-1.518574 \quad-0.539144 \quad-1.089870$

$\begin{array}{llll}\text { C } & -2.160947 & 0.538712 & -1.905149\end{array}$

H $\quad-6.294610 \quad 0.473339-1.515326$

$\begin{array}{llll}\mathrm{H} & -3.939724 & 1.530300 & -2.557389\end{array}$

H $\quad-4.317288-2.850763 \quad 2.046276$

H $\quad-5.901210-3.371654 \quad 1.446142$

H $\quad-7.086674 \quad-1.376641 \quad-0.005002$

H $\quad-2.035003 \quad-2.228748 \quad 0.249905$

H $\quad-1.003861 \quad-1.209824 \quad-1.804330$

$\begin{array}{llll}\mathrm{H} & -1.499965 & 1.188129 & -2.468717\end{array}$

$\begin{array}{llll}\mathrm{H} & -4.418714 & -3.933438 & 0.654255\end{array}$

N $\quad 0.411151 \quad 2.171135 \quad-0.902376$

C $2.930070 \quad-1.608562 \quad 0.817419$

$\begin{array}{llll}\text { O } & 2.713867 & -2.766109 & 1.176857\end{array}$

$\begin{array}{llll}\text { C } & 1.821497 & -0.550925 & 0.777156\end{array}$

C $\quad-0.570374 \quad 3.548170 \quad 0.963059$

C $\quad 4.396394 \quad 0.225560 \quad-0.041922$

$\begin{array}{llll}\text { C } & 5.677309 & 0.072355 & -0.874022\end{array}$

C $6.431205-1.050119 \quad-0.139169$

C $5.322973 \quad-2.015876 \quad 0.315540$

$\begin{array}{llll}\text { C } & 3.204720 & 0.803108 & -0.802065\end{array}$

$\begin{array}{llll}\text { C } & 0.421922 & -1.128088 & 0.538595\end{array}$

$\begin{array}{llll}\text { C } & -0.343655 & 0.013358 & -0.170655\end{array}$

C $\quad 0.746260 \quad 0.759845 \quad-1.015439$

$\begin{array}{llll}\text { C } & -0.300573 & 2.356339 & 0.289966\end{array}$

$\begin{array}{llll}\text { C } & -0.805694 & 1.130001 & 0.756669\end{array}$

C $\quad-1.594022 \quad 1.084204 \quad 1.900962$

C $\quad-1.873019 \quad 2.274665 \quad 2.585310$

$\begin{array}{llll}\text { C } & -1.361803 & 3.488679 & 2.114921\end{array}$

$\begin{array}{llll}\mathrm{N} & 1.999859 & 0.370399 & -0.353815\end{array}$

$\begin{array}{llll}\mathrm{N} & 4.138339 & -1.147837 & 0.431055\end{array}$

$\begin{array}{llll}\mathrm{O} & 3.324239 & 1.606783 & -1.726776\end{array}$

H $\quad 1.163233 \quad 2.810349 \quad-1.137089$

$\begin{array}{llll}\mathrm{H} & 1.846015 & 0.006585 & 1.725035\end{array}$

$\begin{array}{llll}\mathrm{H} & -0.173798 & 4.493987 & 0.605835\end{array}$

$\begin{array}{llll}H & 4.585667 & 0.887280 & 0.818737\end{array}$ 


$\begin{array}{rrrr}\mathrm{H} & 6.239544 & 1.006379 & -0.934107 \\ \mathrm{H} & 5.416362 & -0.232292 & -1.893098 \\ \mathrm{H} & 6.955170 & -0.643242 & 0.732794 \\ \mathrm{H} & 7.171380 & -1.545374 & -0.772529 \\ \mathrm{H} & 5.535184 & -2.500659 & 1.272690 \\ \mathrm{H} & 5.126546 & -2.802344 & -0.422599 \\ \mathrm{H} & -0.048612 & -1.432863 & 1.475099 \\ \mathrm{H} & 0.499505 & -2.011127 & -0.104720 \\ \mathrm{H} & 0.800100 & 0.467323 & -2.067675 \\ \mathrm{H} & -1.982723 & 0.140223 & 2.275221 \\ \mathrm{H} & -2.480487 & 2.252143 & 3.484775 \\ \mathrm{H} & -1.577357 & 4.406476 & 2.655127\end{array}$

Compound R-V

02

$\begin{array}{llll}\mathrm{N} & -3.049957 & 1.767668 & -0.938990\end{array}$

C $\quad 1.226975 \quad-0.379821 \quad 1.350606$

$\begin{array}{llll}\text { O } & 0.668817 & -1.205515 & 2.078688\end{array}$

C $\quad 0.891815 \quad 1.119025 \quad 1.446887$

$\begin{array}{llll}\text { C } & -3.937156 & -0.528659 & -1.404071\end{array}$

$\begin{array}{llll}\text { C } & 2.951727 & 0.232762 & -0.356465\end{array}$

C $3.358945-0.614142 \quad-1.568735$

$\begin{array}{llll}\text { C } & 3.598159 & -1.999161 & -0.939197\end{array}$

$\begin{array}{llll}\text { C } & 2.528685 & -2.114275 & 0.165686\end{array}$

C $\quad 2.191476 \quad 1.510604 \quad-0.674993$

$\begin{array}{llll}\text { C } & -0.590898 & 1.344209 & 1.848498\end{array}$

$\begin{array}{llll}\text { C } & -1.600186 & 1.142981 & 0.761433\end{array}$

$\begin{array}{llll}\text { C } & -2.163567 & 2.189106 & -0.058441\end{array}$

$\begin{array}{llll}\text { C } & -3.128193 & 0.370188 & -0.735631\end{array}$

$\begin{array}{llll}\text { C } & -2.247992 & -0.055782 & 0.304737\end{array}$

$\begin{array}{llll}\text { C } & -2.194798 & -1.406082 & 0.671805\end{array}$

$\begin{array}{llll}\text { C } & -3.019767 & -2.316515 & -0.008681\end{array}$

$\begin{array}{llll}\text { C } & -3.873622 & -1.888708 & -1.027255\end{array}$

$\begin{array}{llll}\mathrm{N} & 1.213738 & 1.831963 & 0.211707\end{array}$

$\begin{array}{llll}\mathrm{N} & 2.173231 & -0.713534 & 0.453780\end{array}$

$\begin{array}{llll}\mathrm{O} & 2.474247 & 2.204186 & -1.647704\end{array}$

H $\quad 1.499014 \quad 1.511817 \quad 2.277799$

H $\quad-4.604721 \quad-0.196304 \quad-2.193602$

$\begin{array}{llll}\mathrm{H} & 3.855768 & 0.535333 & 0.197462\end{array}$

$\begin{array}{llll}\mathrm{H} & 4.236716 & -0.209331 & -2.076008\end{array}$

H $2.532376 \quad-0.644972 \quad-2.286978$

$\begin{array}{llll}\mathrm{H} & 4.599817 & -2.040306 & -0.497273\end{array}$

H $3.522188-2.811683 \quad-1.666205$

H $2.895304 \quad-2.612983 \quad 1.068029$

H $\quad 1.631644 \quad-2.646631 \quad-0.168840$

$\begin{array}{llll}\mathrm{H} & -0.676916 & 2.370569 & 2.224488\end{array}$

H $\quad-0.783836 \quad 0.667611 \quad 2.685956$

$\begin{array}{llll}\mathrm{H} & -1.913981 & 3.244200 & 0.022986\end{array}$

H $\quad-1.518446 \quad-1.741265 \quad 1.451010$

$\begin{array}{llll}\mathrm{H} & -2.991264 & -3.368044 & 0.261809\end{array}$

H $\quad-4.502380-2.611211 \quad-1.539980$

$\begin{array}{llll}H & 0.765510 & 2.726764 & 0.048899\end{array}$

Transition State from $\mathbf{R}-\mathbf{V}$ to $\mathbf{R}-\mathbf{V} \mathbf{I}$

02

\begin{tabular}{|c|c|c|c|}
\hline & 368 & 0 & \\
\hline & -3.057800 & -0.383152 & \\
\hline & -3.737536 & & \\
\hline & -1.622581 & -0.692437 & \\
\hline & 2875 & 637 & 1784 \\
\hline & -2.761023 & -2.328535 & -0.2 \\
\hline & -3.469395 & 9941 & 3383 \\
\hline & -4.936073 & 2684 & 6950 \\
\hline & -4.846998 & 538 & -0.2 \\
\hline & -1.277190 & -2.000168 & 2929 \\
\hline & -0.921317 & & 5086 \\
\hline & -0.165289 & & 5689 \\
\hline & 1.205666 & & 5502 \\
\hline & 0.46 & & 023 \\
\hline C & -0.678346 & & 738 \\
\hline & & & \\
\hline & $-2.0 \varepsilon$ & & 961 \\
\hline & & & \\
\hline & & & \\
\hline & -3 & & \\
\hline & -0.5 & -2.4 & 574 \\
\hline & -1.72 & -1 & \\
\hline & 1.2 & & 45 \\
\hline & -2.8 & -3 & 0. \\
\hline & -3.3 & -3 & -1 \\
\hline & -3.0 & -1 & -2 \\
\hline & -5 & -3 & -0 \\
\hline & -5 & -2 & -2 \\
\hline & -5.6 & & \\
\hline & $-4 . \varepsilon$ & -0 . & -0 . \\
\hline & -0.2 & & \\
\hline & -1.7 & & \\
\hline & & & \\
\hline & -2.8 & & \\
\hline & -3.0 & & -1 \\
\hline & $-1 .(\mathrm{c})$ & & \\
\hline & & -0 & \\
\hline & $2.2 \varepsilon$ & -2. & -1 \\
\hline & 2.27 & & 96 \\
\hline & 5.32 & -2.8 & 720 \\
\hline & 4.18 & -2.2 & 777 \\
\hline & 3.120222 & -2.9 & 244 \\
\hline & 2.76 & -0.7 & $-0 . \varepsilon$ \\
\hline & 3.968233 & -0.8 & -0.0 \\
\hline & 4.673715 & 0.3 & 90 \\
\hline & 4.167951 & 1.5 & -0.2 \\
\hline & 2.904802 & 1.6 & $-0 . \varsigma$ \\
\hline & 1.322837 & -2.2 & -1.2 \\
\hline & 1.38 & 0.4 & -1 \\
\hline & 5.1 & -3.9 & \\
\hline & 6.2 & -2.6 & \\
\hline & & & \\
\hline & & & \\
\hline & & & \\
\hline & 2.774659 & 2.5 & 15 \\
\hline
\end{tabular}




$\begin{array}{rrrr}\mathrm{H} & -5.866236 & -3.553292 & 1.944514 \\ \mathrm{H} & -3.625863 & -1.726528 & -2.350738 \\ \mathrm{H} & -2.728537 & 0.556357 & -2.347430 \\ \mathrm{H} & -3.769090 & 1.993874 & -0.530962 \\ \mathrm{H} & -5.657401 & -3.838633 & -1.780837\end{array}$

Compound R-VI

02

\begin{tabular}{|c|c|c|c|}
\hline & & & \\
\hline & & & \\
\hline & & & \\
\hline & & 892 & \\
\hline & & 3849 & 180 \\
\hline & 828 & 5469 & \\
\hline & 7536 & 7464 & \\
\hline & 5048 & 1468 & \\
\hline & 7411 & 3262 & \\
\hline & 764 & 830 & 302 \\
\hline & 2130 & & \\
\hline & & & \\
\hline & & & \\
\hline & & & \\
\hline & & & \\
\hline & & & \\
\hline & & & \\
\hline & & & \\
\hline & & & \\
\hline & & & \\
\hline & & & \\
\hline & & & \\
\hline & & & \\
\hline & & & \\
\hline & & & \\
\hline & & & \\
\hline & & & \\
\hline & & & \\
\hline & & & \\
\hline & & & \\
\hline & & & \\
\hline & & & \\
\hline & & & \\
\hline & & & \\
\hline & & & \\
\hline & & & \\
\hline & & & \\
\hline & & & \\
\hline 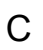 & & & \\
\hline 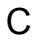 & & -4 & \\
\hline 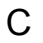 & 682 & -2.8 & \\
\hline C & & -2 & \\
\hline & -4.4 & $-0 . \varepsilon$ & \\
\hline C & 024 & 7306 & \\
\hline & & 289 & \\
\hline & & 3626 & \\
\hline & -3.2 & 678 & -0.2 \\
\hline & & -1.2 & \\
\hline & & & \\
\hline & & & \\
\hline & -5.577097 & -4.90 & -0.3 \\
\hline
\end{tabular}

$\begin{array}{lrrr}\text { Compound PDT } & & \\ \text { O 1 } & & & \\ \text { N } & 1.356808 & 0.707155 & 0.573365 \\ \mathrm{C} & -4.081042 & 0.336718 & 0.949207 \\ \mathrm{O} & -4.341482 & 1.528507 & 1.137207 \\ \mathrm{C} & -3.008470 & -0.408945 & 1.766030 \\ \mathrm{C} & 1.096272 & 2.383042 & -1.305989 \\ \mathrm{C} & -4.510119 & -1.867295 & -0.146330 \\ \mathrm{C} & -5.062732 & -2.108654 & -1.555841 \\ \mathrm{C} & -6.245956 & -1.125921 & -1.631076 \\ \mathrm{C} & -5.763561 & 0.113109 & -0.853560 \\ \mathrm{C} & -3.043113 & -2.235749 & 0.048003 \\ \mathrm{C} & -1.917459 & 0.538487 & 2.333466 \\ \mathrm{C} & -0.748562 & 0.826435 & 1.431506 \\ \mathrm{C} & 0.494137 & 0.254674 & 1.560531 \\ \mathrm{C} & 0.660142 & 1.614018 & -0.219437 \\ \mathrm{C} & -0.665674 & 1.710617 & 0.291072 \\ \mathrm{C} & -1.570271 & 2.595368 & -0.325835 \\ \mathrm{C} & -1.141789 & 3.349713 & -1.411588 \\ \mathrm{C} & 0.179071 & 3.246737 & -1.894550 \\ \mathrm{~N} & -2.411833 & -1.496753 & 0.992314 \\ \mathrm{~N} & -4.740787 & -0.427444 & 0.056384 \\ \mathrm{O} & -2.502081 & -3.137109 & -0.588784 \\ \mathrm{H} & -3.545340 & -0.826325 & 2.633463 \\ \mathrm{H} & 2.115685 & 2.313803 & -1.670960 \\ \mathrm{H} & -5.095666 & -2.444063 & 0.588558 \\ \mathrm{H} & -5.353094 & -3.149981 & -1.710331 \\ \mathrm{H} & -4.295071 & -1.857647 & -2.295472 \\ \mathrm{H} & -7.124714 & -1.558408 & -1.139529 \\ \mathrm{H} & -6.526649 & -0.880333 & -2.658555 \\ \mathrm{H} & -6.559636 & 0.605398 & -0.286949 \\ \mathrm{H} & -5.305253 & 0.864485 & -1.506958 \\ \mathrm{H} & -1.537527 & 0.087060 & 3.256466 \\ \mathrm{H} & -2.434722 & 1.460446 & 2.616301 \\ \mathrm{H} & 0.847396 & -0.461960 & 2.290180 \\ \mathrm{H} & -2.586256 & 2.680368 & 0.046491 \\ \mathrm{H} & -1.833111 & 4.035049 & -1.894955 \\ \mathrm{H} & 0.489639 & 3.855495 & -2.739530 \\ \mathrm{H} & -1.416606 & -1.660170 & 1.099554 \\ \mathrm{~N} & 5.259044 & -0.997053 & -1.940452 \\ \mathrm{C} & 3.187533 & -0.137795 & -0.799451 \\ \mathrm{C} & 7.916480 & -1.150499 & 0.656990 \\ \mathrm{C} & 6.682845 & -0.992579 & -0.181149 \\ \mathrm{C} & 6.548326 & -1.273608 & -1.518287 \\ \mathrm{C} & 4.527773 & -0.528064 & -0.873914 \\ & 5.395085 & -0.512776 & 0.256543 \\ \mathrm{C} & 4.882918 & -0.072616 & 1.489204 \\ & 0.339613 & 1.571412 \\ & 0.303755 & 0.436455\end{array}$




$\begin{array}{rrrr}\mathrm{H} & 4.913928 & -1.125835 & -2.879716 \\ \mathrm{H} & 2.528304 & -0.191921 & -1.659576 \\ \mathrm{H} & 7.754077 & -1.842854 & 1.493444 \\ \mathrm{H} & 8.749271 & -1.539320 & 0.062038 \\ \mathrm{H} & 7.279620 & -1.655734 & -2.217705 \\ \mathrm{H} & 5.516089 & -0.040955 & 2.371856 \\ \mathrm{H} & 3.159007 & 0.709255 & 2.510163 \\ \mathrm{H} & 8.237153 & -0.195091 & 1.092582\end{array}$

Compound C-V

11

$\begin{array}{llll}\mathrm{N} & 2.390732 & -1.819704 & -1.422595\end{array}$

$\begin{array}{llll}\text { C } & -1.008776 & 0.551870 & 1.251532\end{array}$

$\begin{array}{llll}\text { O } & -0.197445 & 1.319865 & 1.779028\end{array}$

$\begin{array}{llll}\text { C } & -0.945713 & -0.960549 & 1.542905\end{array}$

$\begin{array}{llll}\text { C } & 3.836111 & 0.234091 & -1.555774\end{array}$

C $\quad-3.002929 \quad 0.089168 \quad-0.185243$

$\begin{array}{llll}\text { C } & -3.345418 & 0.815108 & -1.493051\end{array}$

$\begin{array}{llll}\text { C } & -3.234871 & 2.295983 & -1.084955\end{array}$

$\begin{array}{llll}\text { C } & -2.061525 & 2.340070 & -0.085488\end{array}$

C $\quad-2.579796-1.362179 \quad-0.323993$

$\begin{array}{llll}\text { C } & 0.545899 & -1.380014 & 1.793450\end{array}$

C $\quad 1.424478-1.2169820 .619718$

C $1.542912-2.192876 \quad-0.530695$

$\begin{array}{llll}\text { C } & 2.909647 & -0.559881 & -0.953304\end{array}$

$\begin{array}{llll}\text { C } & 2.313732 & -0.191979 & 0.296969\end{array}$

C $2.667406 \quad 1.015553 \quad 0.947923$

$\begin{array}{llll}\text { C } & 3.618429 & 1.835931 & 0.332323\end{array}$

C $\quad 4.186644 \quad 1.453287 \quad-0.881786$

$\begin{array}{llll}\mathrm{N} & -1.550420 & -1.760995 & 0.489326\end{array}$

$\begin{array}{llll}\mathrm{N} & -1.966607 & 0.949522 & 0.402982\end{array}$

$\begin{array}{llll}\mathrm{O} & -3.151157 & -2.130201 & -1.084283\end{array}$

H $\quad-1.445616 \quad-1.134175 \quad 2.507029$

H $4.297364 \quad-0.029133 \quad-2.500840$

$\begin{array}{llll}\text { H } & -3.887882 & 0.084951 & 0.470995\end{array}$

H $\quad-4.333305 \quad 0.539465 \quad-1.865838$

$\begin{array}{llll}\mathrm{H} & -2.605326 & 0.560458 & -2.259565\end{array}$

H $\quad-4.158645 \quad 2.618336 \quad-0.593097$

$\begin{array}{llll}\mathrm{H} & -3.064821 & 2.955626 & -1.939090\end{array}$

$\begin{array}{llll}\mathrm{H} & -2.230382 & 3.026922 & 0.748602\end{array}$

$\begin{array}{llll}\mathrm{H} & -1.111989 & 2.612101 & -0.558233\end{array}$

$\begin{array}{llll}H & 0.539869 & -2.435455 & 2.093546\end{array}$

H $0.897858 \quad-0.772637 \quad 2.628174$

H $\quad 0.983796 \quad-3.119325 \quad-0.605294$

$\begin{array}{llll}H & 2.195807 & 1.295638 & 1.882198\end{array}$

$\begin{array}{llll}H & 3.915654 & 2.768208 & 0.798024\end{array}$

H $4.926974 \quad 2.099349 \quad-1.343953$

H $\quad-1.443865 \quad-2.768993 \quad 0.548216$

Iron-Oxo Quartet FeO_quartet

04
$\begin{array}{llll}\text { Fe } & 0.089141 & 0.036057 & -0.377911\end{array}$
N $\quad \begin{array}{llll}1.819494 & -0.979714 & -0.146250\end{array}$
$\begin{array}{llll}\mathrm{N} & -0.917496 & -1.709275 & -0.274068\end{array}$
$\begin{array}{llll}\mathrm{N} & 1.083957 & 1.774351 & -0.133367\end{array}$

$\begin{array}{lrrr}\text { N } & -1.657172 & 1.037712 & -0.294031 \\ \mathrm{C} & 3.081401 & -0.442680 & -0.039450 \\ \mathrm{C} & -2.283740 & -1.880043 & -0.331182 \\ \mathrm{C} & 1.995090 & -2.338860 & -0.207619 \\ \mathrm{C} & -0.378864 & -2.980953 & -0.309906 \\ \mathrm{C} & 2.440098 & 1.939745 & -0.026959 \\ \mathrm{C} & -2.917859 & 0.504666 & -0.369167 \\ \mathrm{C} & 0.552637 & 3.037947 & -0.164823 \\ \mathrm{C} & -1.825001 & 2.402919 & -0.311109 \\ \mathrm{C} & 4.071808 & -1.491461 & -0.023063 \\ \mathrm{C} & -2.610089 & -3.284464 & -0.372069 \\ \mathrm{C} & 3.397629 & -2.668854 & -0.134568 \\ \mathrm{C} & -1.431248 & -3.964519 & -0.364664 \\ \mathrm{C} & 2.774252 & 3.342623 & 0.015773 \\ \mathrm{C} & -3.906480 & 1.553781 & -0.424663 \\ \mathrm{C} & 1.599634 & 4.026212 & -0.073553 \\ \mathrm{C} & -3.227094 & 2.733367 & -0.389255 \\ \mathrm{H} & 5.138437 & -1.328178 & 0.061663 \\ \mathrm{H} & -3.617167 & -3.679288 & -0.410096 \\ \mathrm{H} & 3.794198 & -3.675647 & -0.157896 \\ \mathrm{H} & -1.269081 & -5.034293 & -0.392542 \\ \mathrm{H} & 3.779796 & 3.733563 & 0.102573 \\ \mathrm{H} & -4.974948 & 1.389913 & -0.480023 \\ \mathrm{H} & 1.437863 & 5.096418 & -0.073688 \\ \mathrm{H} & -3.621023 & 3.741304 & -0.409414 \\ \mathrm{C} & 3.374694 & 0.911083 & 0.028832 \\ \mathrm{C} & -3.216766 & -0.854725 & -0.375471 \\ \mathrm{C} & -0.802078 & 3.337879 & -0.250908 \\ \mathrm{C} & 0.973072 & -3.277374 & -0.299936 \\ \mathrm{H} & 4.419583 & 1.191446 & 0.116024 \\ \mathrm{H} & -4.263773 & -1.135122 & -0.431928 \\ \mathrm{H} & -1.081791 & 4.386473 & -0.266560 \\ \mathrm{H} & 1.257039 & -4.323926 & -0.342683 \\ \mathrm{O} & 0.174206 & 0.077727 & -2.000977 \\ \mathrm{~S} & -0.029871 & -0.377906 & 2.210114 \\ \mathrm{C} & -1.717070 & -0.057908 & 2.807120 \\ \mathrm{H} & -2.414815 & -0.741276 & 2.308018 \\ \mathrm{H} & -1.755821 & -0.254933 & 3.882505 \\ \mathrm{H} & -2.027517 & 0.968924 & 2.599579\end{array}$

Iron-Hydroxo Triplet

03

$\begin{array}{llll}\text { Fe } & 0.057031 & 0.006037 & -0.226647\end{array}$

N $\quad 0.974394 \quad-1.797923 \quad-0.242753$

$\begin{array}{llll}\mathrm{N} & -1.731268 & -0.907687 & -0.220318\end{array}$

$\begin{array}{llll}N & 1.856372 & 0.908100 & -0.084704\end{array}$

$\begin{array}{llll}\mathrm{N} & -0.852320 & 1.799772 & -0.277175\end{array}$

C $\quad 2.329800 \quad-2.034563 \quad-0.206678$

C $\quad-2.960752 \quad-0.301749-0.339972$

$\begin{array}{llll}\text { C } & 0.367226 & -3.032337 & -0.203144\end{array}$

C $\quad-1.968581 \quad-2.262094 \quad-0.209979$

C $\quad 3.088172 \quad 0.302557 \quad-0.085169$

C $\quad-2.199602 \quad 2.037186 \quad-0.378754$

$\begin{array}{llll}\text { C } & 2.088668 & 2.262332 & -0.048308\end{array}$

C $\quad-0.244044 \quad 3.029094 \quad-0.224220$ 


$\begin{array}{rrrr}\mathrm{C} & 2.580119 & -3.454499 & -0.182363 \\ \mathrm{C} & -3.998864 & -1.300172 & -0.375981 \\ \mathrm{C} & 1.366010 & -4.071634 & -0.175489 \\ \mathrm{C} & -3.385332 & -2.513778 & -0.289586 \\ \mathrm{C} & 4.126445 & 1.301639 & -0.022854 \\ \mathrm{C} & -2.448488 & 3.458318 & -0.407465 \\ \mathrm{C} & 3.507320 & 2.514797 & 0.003547 \\ \mathrm{C} & -1.237045 & 4.072641 & -0.307636 \\ \mathrm{H} & 3.565438 & -3.902074 & -0.160285 \\ \mathrm{H} & -5.055580 & -1.082119 & -0.462645 \\ \mathrm{H} & 1.147003 & -5.131360 & -0.149657 \\ \mathrm{H} & -3.833148 & -3.499210 & -0.293325 \\ \mathrm{H} & 5.186599 & 1.083695 & -0.008209 \\ \mathrm{H} & -3.429966 & 3.907778 & -0.487049 \\ \mathrm{H} & 3.953478 & 3.500219 & 0.041649 \\ \mathrm{H} & -1.015958 & 5.132094 & -0.290082 \\ \mathrm{C} & 3.319890 & -1.065283 & -0.147197 \\ \mathrm{C} & -3.189691 & 1.064480 & -0.417758 \\ \mathrm{C} & 1.120128 & 3.254418 & -0.104192 \\ \mathrm{C} & -1.000845 & -3.257576 & -0.181502 \\ \mathrm{H} & 4.351412 & -1.401018 & -0.128845 \\ \mathrm{H} & -4.218904 & 1.396951 & -0.503597 \\ \mathrm{H} & 1.456901 & 4.285330 & -0.071754 \\ \mathrm{H} & -1.340819 & -4.287758 & -0.163832 \\ \mathrm{O} & 0.105832 & 0.052682 & -2.029855 \\ \mathrm{~S} & 0.134786 & 0.070941 & 2.082629 \\ \mathrm{C} & -1.551017 & -0.093903 & 2.771846 \\ \mathrm{H} & -1.987130 & -1.064516 & 2.525644 \\ \mathrm{H} & -1.465322 & -0.001876 & 3.858606 \\ \mathrm{H} & -2.198792 & 0.701402 & 2.395289 \\ \mathrm{H} & 0.411430 & -0.826094 & -2.320515 \\ & & & \end{array}$

Iron-Aqua Doublet

02

$\begin{array}{lrrr}\text { Fe } & -0.061810 & -0.012974 & -0.108940 \\ \mathrm{~N} & -1.440696 & 1.471790 & -0.171790 \\ \mathrm{~N} & 1.426825 & 1.340160 & -0.271464 \\ \mathrm{~N} & -1.567654 & -1.350895 & -0.149754 \\ \mathrm{~N} & 1.297105 & -1.480332 & -0.269235 \\ \mathrm{C} & -2.811606 & 1.352528 & -0.123321 \\ \mathrm{C} & 2.780244 & 1.098135 & -0.365256 \\ \mathrm{C} & -1.176848 & 2.822091 & -0.121987 \\ \mathrm{C} & 1.290574 & 2.709063 & -0.215092 \\ \mathrm{C} & -2.919623 & -1.107179 & -0.115452 \\ \mathrm{C} & 2.663493 & -1.361298 & -0.376448 \\ \mathrm{C} & -1.428734 & -2.716964 & -0.114451 \\ \mathrm{C} & 1.036251 & -2.828918 & -0.228404 \\ \mathrm{C} & -3.424222 & 2.657030 & -0.063240 \\ \mathrm{C} & 3.509577 & 2.342040 & -0.374909 \\ \mathrm{C} & -2.411019 & 3.567883 & -0.059073 \\ \mathrm{C} & 2.586833 & 3.340253 & -0.276853 \\ \mathrm{C} & -3.651620 & -2.351266 & -0.067339 \\ \mathrm{C} & 3.277292 & -2.667368 & -0.409065 \\ \mathrm{C} & -2.726705 & -3.350030 & -0.063671 \\ \mathrm{C} & 2.268486 & -3.577279 & -0.312873\end{array}$

$\begin{array}{rrrr}\mathrm{H} & -4.491511 & 2.834722 & -0.025654 \\ \mathrm{H} & 4.586746 & 2.423825 & -0.447020 \\ \mathrm{H} & -2.475562 & 4.647925 & -0.018730 \\ \mathrm{H} & 2.751516 & 4.410129 & -0.254024 \\ \mathrm{H} & -4.731062 & -2.429621 & -0.039679 \\ \mathrm{H} & 4.341759 & -2.845496 & -0.494230 \\ \mathrm{H} & -2.888531 & -4.420111 & -0.034299 \\ \mathrm{H} & 2.333066 & -4.658000 & -0.304806 \\ \mathrm{C} & -3.508948 & 0.151165 & -0.110512 \\ \mathrm{C} & 3.362852 & -0.161422 & -0.429642 \\ \mathrm{C} & -0.224180 & -3.408337 & -0.145828 \\ \mathrm{C} & 0.086616 & 3.400029 & -0.138759 \\ \mathrm{H} & -4.592712 & 0.199035 & -0.073877 \\ \mathrm{H} & 4.444266 & -0.212462 & -0.508450 \\ \mathrm{H} & -0.271881 & -4.492591 & -0.120154 \\ \mathrm{H} & 0.137795 & 4.483815 & -0.100804 \\ \mathrm{O} & -0.145349 & 0.032792 & -2.296283 \\ \mathrm{~S} & -0.085960 & -0.034935 & 2.133451 \\ \mathrm{C} & 1.638559 & 0.015887 & 2.746327 \\ \mathrm{H} & 2.132588 & 0.951395 & 2.472574 \\ \mathrm{H} & 1.591581 & -0.054151 & 3.837830 \\ \mathrm{H} & 2.222347 & -0.824329 & 2.363104 \\ \mathrm{H} & -0.898807 & 0.632536 & -2.442733 \\ \mathrm{H} & 0.640921 & 0.566030 & -2.510789\end{array}$

Iron-Hydroxo Doublet Anion $-12$

$\begin{array}{lrrr}\text { Fe } & 0.064821 & -0.001115 & -0.221145 \\ \text { N } & 1.585262 & -1.345021 & -0.150582 \\ \mathrm{~N} & -1.305723 & -1.489454 & -0.267154 \\ \mathrm{~N} & 1.446338 & 1.486933 & -0.159251 \\ \mathrm{~N} & -1.443693 & 1.345391 & -0.276697 \\ \mathrm{C} & 2.931596 & -1.096136 & -0.097124 \\ \mathrm{C} & -2.665785 & -1.371806 & -0.365284 \\ \mathrm{C} & 1.443391 & -2.708095 & -0.142425 \\ \mathrm{C} & -1.032427 & -2.830214 & -0.254517 \\ \mathrm{C} & 2.810444 & 1.370747 & -0.105622 \\ \mathrm{C} & -2.786159 & 1.094913 & -0.373573 \\ \mathrm{C} & 1.172035 & 2.829343 & -0.161717 \\ \mathrm{C} & -1.303741 & 2.706417 & -0.270540 \\ \mathrm{C} & 3.667576 & -2.341348 & -0.056227 \\ \mathrm{C} & -3.278135 & -2.683455 & -0.407602 \\ \mathrm{C} & 2.743649 & -3.342022 & -0.083157 \\ \mathrm{C} & -2.263598 & -3.589312 & -0.336544 \\ \mathrm{C} & 3.421566 & 2.682362 & -0.074348 \\ \mathrm{C} & -3.524068 & 2.339897 & -0.421777 \\ \mathrm{C} & 2.404506 & 3.587718 & -0.107798 \\ \mathrm{C} & -2.603301 & 3.341178 & -0.355152 \\ \mathrm{H} & 4.747013 & -2.419808 & -0.011309 \\ \mathrm{H} & -4.343201 & -2.866275 & -0.482376 \\ \mathrm{H} & 2.907871 & -4.412600 & -0.065784 \\ \mathrm{H} & -2.322694 & -4.670947 & -0.342212 \\ \mathrm{H} & 4.488240 & 2.865889 & -0.030976 \\ \mathrm{H} & -4.601947 & 2.417145 & -0.496777 \\ \mathrm{H} & 2.463382 & 4.669324 & -0.098415\end{array}$




$\begin{array}{rrrr}\mathrm{H} & -2.767940 & 4.411805 & -0.365729 \\ \mathrm{C} & 3.512974 & 0.169140 & -0.078506 \\ \mathrm{C} & -3.365870 & -0.169537 & -0.418899 \\ \mathrm{C} & -0.096751 & 3.399739 & -0.211639 \\ \mathrm{C} & 0.236567 & -3.400673 & -0.190315 \\ \mathrm{H} & 4.596912 & 0.222512 & -0.034853 \\ \mathrm{H} & -4.448002 & -0.222606 & -0.497209 \\ \mathrm{H} & -0.150258 & 4.484860 & -0.211157 \\ \mathrm{H} & 0.290072 & -4.485778 & -0.183511 \\ \mathrm{O} & 0.110636 & -0.002976 & -2.069830 \\ \mathrm{~S} & 0.066238 & -0.018863 & 2.182733 \\ \mathrm{C} & -1.665805 & 0.046371 & 2.792667 \\ \mathrm{H} & -2.255038 & -0.791062 & 2.406404 \\ \mathrm{H} & -1.659807 & -0.008823 & 3.886918 \\ \mathrm{H} & -2.159557 & 0.977055 & 2.495720 \\ \mathrm{H} & 1.057433 & -0.012378 & -2.287687\end{array}$

Figure 11B Radical C2 TS 02

$\begin{array}{lrrr}\mathrm{N} & 2.035711 & -0.920612 & 1.727039 \\ \mathrm{C} & 2.372037 & -3.391249 & 1.368746 \\ \mathrm{C} & 4.189336 & 0.763761 & -0.802561 \\ \mathrm{C} & 3.379826 & -0.147172 & 0.065864 \\ \mathrm{C} & 2.436656 & 0.243184 & 1.056341 \\ \mathrm{C} & 2.556205 & -2.033040 & 1.103180 \\ \mathrm{C} & 3.406556 & -1.572232 & 0.050721 \\ \mathrm{C} & 4.089040 & -2.522830 & -0.737597 \\ \mathrm{C} & 3.912805 & -3.875255 & -0.469107 \\ \mathrm{C} & 3.063223 & -4.304924 & 0.571702 \\ \mathrm{H} & 1.420774 & -0.942104 & 2.525591 \\ \mathrm{H} & 1.719648 & -3.725291 & 2.170809 \\ \mathrm{H} & 3.880152 & 0.715524 & -1.856614 \\ \mathrm{H} & 4.103793 & 1.805800 & -0.482330 \\ \mathrm{H} & 2.487971 & 1.165416 & 1.623048 \\ \mathrm{H} & 4.742380 & -2.200202 & -1.543980 \\ \mathrm{H} & 4.433374 & -4.615938 & -1.070007 \\ \mathrm{H} & 2.944094 & -5.368648 & 0.758460 \\ \mathrm{H} & 5.252058 & 0.488585 & -0.776539 \\ \mathrm{~N} & -0.479438 & 2.379289 & 1.571440 \\ \mathrm{C} & -2.034364 & -1.179002 & -1.412882 \\ \mathrm{O} & -1.542500 & -2.038164 & -2.145616 \\ \mathrm{C} & -1.280255 & 0.095175 & -1.026703 \\ \mathrm{C} & 0.104475 & 4.583270 & 0.515677 \\ \mathrm{C} & -3.876945 & -0.259676 & 0.007742 \\ \mathrm{C} & -4.967803 & -1.054993 & 0.738210 \\ \mathrm{C} & -5.430459 & -2.063763 & -0.328185 \\ \mathrm{C} & -4.139614 & -2.442450 & -1.075697 \\ \mathrm{C} & -2.829692 & 0.381147 & 0.918333 \\ \mathrm{C} & 0.258567 & -0.047518 & -0.992025 \\ \mathrm{C} & 0.664288 & 0.984132 & 0.038824 \\ \mathrm{C} & -0.432701 & 1.004446 & 1.109015 \\ \mathrm{C} & 0.124195 & 3.194242 & 0.613673 \\ \mathrm{C} & 0.838169 & 2.393779 & -0.317131 \\ \mathrm{C} & 1.528179 & 3.014941 & -1.364245 \\ \mathrm{C} & 1.513452 & 4.410286 & -1.468951\end{array}$

$\begin{array}{rrrr}\mathrm{C} & 0.811586 & 5.182611 & -0.535799 \\ \mathrm{~N} & -1.615213 & 0.530045 & 0.344067 \\ \mathrm{~N} & -3.278971 & -1.263776 & -0.891117 \\ \mathrm{O} & -3.083078 & 0.745757 & 2.069219 \\ \mathrm{H} & -1.326120 & 2.675432 & 2.041597 \\ \mathrm{H} & -1.560198 & 0.882542 & -1.742760 \\ \mathrm{H} & -0.454851 & 5.185472 & 1.226517 \\ \mathrm{H} & -4.335783 & 0.547580 & -0.586220 \\ \mathrm{H} & -5.770313 & -0.410498 & 1.103314 \\ \mathrm{H} & -4.528169 & -1.568296 & 1.600071 \\ \mathrm{H} & -6.135602 & -1.585006 & -1.017051 \\ \mathrm{H} & -5.926492 & -2.937394 & 0.102781 \\ \mathrm{H} & -4.297250 & -2.639222 & -2.140295 \\ \mathrm{H} & -3.650451 & -3.323245 & -0.642657 \\ \mathrm{H} & 0.700225 & 0.126688 & -1.975135 \\ \mathrm{H} & 0.523813 & -1.062237 & -0.677391 \\ \mathrm{H} & -0.304464 & 0.327653 & 1.959040 \\ \mathrm{H} & 2.061144 & 2.419589 & -2.100161 \\ \mathrm{H} & 2.045030 & 4.895835 & -2.282438 \\ \mathrm{H} & 0.804682 & 6.265389 & -0.629336\end{array}$

Figure 11B Radical C2 Product 02

$\begin{array}{lrrr}\text { N } & 2.021897 & -0.812981 & 1.380367 \\ \mathrm{C} & 3.475741 & -2.854607 & 1.436932 \\ \mathrm{C} & 3.688043 & 1.336042 & -1.270884 \\ \mathrm{C} & 3.175283 & 0.298457 & -0.326469 \\ \mathrm{C} & 2.050322 & 0.485176 & 0.678402 \\ \mathrm{C} & 3.074558 & -1.614212 & 0.958229 \\ \mathrm{C} & 3.747670 & -0.973253 & -0.128925 \\ \mathrm{C} & 4.826988 & -1.641481 & -0.755068 \\ \mathrm{C} & 5.221157 & -2.889476 & -0.278958 \\ \mathrm{C} & 4.560355 & -3.488268 & 0.807315 \\ \mathrm{H} & 1.800037 & -0.806017 & 2.367699 \\ \mathrm{H} & 2.964921 & -3.323651 & 2.273540 \\ \mathrm{H} & 3.195911 & 1.301554 & -2.254875 \\ \mathrm{H} & 3.547794 & 2.349416 & -0.880310 \\ \mathrm{H} & 2.298025 & 1.295207 & 1.385644 \\ \mathrm{H} & 5.347447 & -1.180416 & -1.590059 \\ \mathrm{H} & 6.052401 & -3.406840 & -0.750372 \\ \mathrm{H} & 4.888869 & -4.459622 & 1.166566 \\ \mathrm{H} & 4.759246 & 1.189607 & -1.454013 \\ \mathrm{~N} & -0.628700 & 1.996562 & 1.787857 \\ \mathrm{C} & -2.056025 & -1.345315 & -1.463782 \\ \mathrm{O} & -1.549091 & -2.096623 & -2.297401 \\ \mathrm{C} & -1.359476 & -0.072008 & -0.975772 \\ \mathrm{C} & -0.605075 & 4.291176 & 0.765246 \\ \mathrm{C} & -3.879092 & -0.678104 & 0.111844 \\ \mathrm{C} & -4.896036 & -1.600332 & 0.798199 \\ \mathrm{C} & -5.351679 & -2.521613 & -0.347142 \\ \mathrm{C} & -4.074839 & -2.755885 & -1.173090 \\ \mathrm{C} & -2.829214 & -0.074632 & 1.043769 \\ \mathrm{C} & 0.172643 & -0.127145 & -1.018184 \\ \mathrm{C} & 0.620261 & 0.830730 & 0.102279 \\ \mathrm{C} & -0.476397 & 0.673631 & 1.207300\end{array}$




$\begin{array}{rrrr}\mathrm{C} & -0.298999 & 2.930872 & 0.798768 \\ \mathrm{C} & 0.441997 & 2.316146 & -0.229516 \\ \mathrm{C} & 0.840931 & 3.070035 & -1.327861 \\ \mathrm{C} & 0.535405 & 4.438275 & -1.379740 \\ \mathrm{C} & -0.173014 & 5.038487 & -0.336083 \\ \mathrm{~N} & -1.646763 & 0.190966 & 0.444607 \\ \mathrm{~N} & -3.270546 & -1.552544 & -0.907423 \\ \mathrm{O} & -3.054254 & 0.163914 & 2.232917 \\ \mathrm{H} & -1.481104 & 2.132029 & 2.320891 \\ \mathrm{H} & -1.724799 & 0.766542 & -1.587790 \\ \mathrm{H} & -1.178719 & 4.753052 & 1.564048 \\ \mathrm{H} & -4.403600 & 0.158402 & -0.378316 \\ \mathrm{H} & -5.713580 & -1.039776 & 1.256362 \\ \mathrm{H} & -4.394655 & -2.172258 & 1.586202 \\ \mathrm{H} & -6.105989 & -2.014621 & -0.959471 \\ \mathrm{H} & -5.786580 & -3.458450 & 0.010712 \\ \mathrm{H} & -4.264819 & -2.860542 & -2.245344 \\ \mathrm{H} & -3.523013 & -3.644316 & -0.843321 \\ \mathrm{H} & 0.560456 & 0.157519 & -1.998437 \\ \mathrm{H} & 0.513263 & -1.143496 & -0.799963 \\ \mathrm{H} & -0.252525 & -0.057497 & 1.984089 \\ \mathrm{H} & 1.371184 & 2.609648 & -2.155130 \\ \mathrm{H} & 0.845026 & 5.027509 & -2.237875 \\ \mathrm{H} & -0.408703 & 6.098494 & -0.383328\end{array}$

Figure 11B Radical C3 TS

$\begin{array}{lrrr}02 & & & \\ \mathrm{~N} & 1.839883 & -2.633470 & 1.532976 \\ \mathrm{C} & 3.492027 & -3.344098 & -0.234524 \\ \mathrm{C} & 2.591438 & 0.876978 & 2.235670 \\ \mathrm{C} & 2.155020 & -0.351744 & 1.458445 \\ \mathrm{C} & 1.502361 & -1.434502 & 2.123310 \\ \mathrm{C} & 2.814221 & -2.424904 & 0.569462 \\ \mathrm{C} & 3.089023 & -1.035329 & 0.531812 \\ \mathrm{C} & 4.111959 & -0.570329 & -0.298739 \\ \mathrm{C} & 4.809425 & -1.481030 & -1.097377 \\ \mathrm{C} & 4.493161 & -2.849822 & -1.070929 \\ \mathrm{H} & 1.437641 & -3.529077 & 1.768168 \\ \mathrm{H} & 3.259837 & -4.404780 & -0.199240 \\ \mathrm{H} & 2.887521 & 1.689315 & 1.566225 \\ \mathrm{H} & 1.787932 & 1.249816 & 2.877810 \\ \mathrm{H} & 0.797672 & -1.405069 & 2.941892 \\ \mathrm{H} & 4.363939 & 0.485654 & -0.324688 \\ \mathrm{H} & 5.607927 & -1.128216 & -1.744005 \\ \mathrm{H} & 5.044675 & -3.539788 & -1.703837 \\ \mathrm{H} & 3.450043 & 0.634201 & 2.873558 \\ \mathrm{~N} & -0.266893 & 2.391172 & 1.215343 \\ \mathrm{C} & -2.301233 & -1.352517 & -1.152531 \\ \mathrm{O} & -1.956889 & -2.434305 & -1.631132 \\ \mathrm{C} & -1.316991 & -0.203124 & -0.920506 \\ \mathrm{C} & 0.810074 & 4.128833 & -0.246056 \\ \mathrm{C} & -3.997217 & 0.208870 & -0.184103 \\ \mathrm{C} & -5.300985 & -0.167547 & 0.533070 \\ \mathrm{C} & -5.890870 & -1.259731 & -0.376613 \\ \mathrm{C} & -4.662769 & -2.050947 & -0.860146\end{array}$

$\begin{array}{rrrr}\text { C } & -2.913375 & 0.796265 & 0.720554 \\ \mathrm{C} & 0.143305 & -0.637826 & -0.680726 \\ \mathrm{C} & 0.700853 & 0.474392 & 0.194302 \\ \mathrm{C} & -0.457701 & 0.955743 & 1.084086 \\ \mathrm{C} & 0.555552 & 2.826334 & 0.174214 \\ \mathrm{C} & 1.172925 & 1.711173 & -0.449840 \\ \mathrm{C} & 2.043836 & 1.923960 & -1.523597 \\ \mathrm{C} & 2.307594 & 3.232078 & -1.950336 \\ \mathrm{C} & 1.698620 & 4.319356 & -1.314873 \\ \mathrm{~N} & -1.653396 & 0.546363 & 0.303697 \\ \mathrm{~N} & -3.573210 & -1.064580 & -0.794504 \\ \mathrm{O} & -3.178219 & 1.455580 & 1.729583 \\ \mathrm{H} & -1.082752 & 2.930917 & 1.478728 \\ \mathrm{H} & -1.367966 & 0.467138 & -1.792297 \\ \mathrm{H} & 0.322051 & 4.974807 & 0.230694 \\ \mathrm{H} & -4.204427 & 0.950434 & -0.972875 \\ \mathrm{H} & -5.960550 & 0.693035 & 0.663026 \\ \mathrm{H} & -5.067988 & -0.567989 & 1.525538 \\ \mathrm{H} & -6.401815 & -0.801868 & -1.231279 \\ \mathrm{H} & -6.612488 & -1.897384 & 0.140825 \\ \mathrm{H} & -4.769085 & -2.437407 & -1.878142 \\ \mathrm{H} & -4.429759 & -2.898583 & -0.204314 \\ \mathrm{H} & 0.685205 & -0.754768 & -1.621031 \\ \mathrm{H} & 0.147450 & -1.602195 & -0.164722 \\ \mathrm{H} & -0.531050 & 0.489819 & 2.071215 \\ \mathrm{H} & 2.503897 & 1.081234 & -2.031654 \\ \mathrm{H} & 2.981453 & 3.401257 & -2.785645 \\ \mathrm{H} & 1.905266 & 5.329701 & -1.658309\end{array}$

Figure 11B Radical C3 Product 02

$\begin{array}{rrrr}\text { N } & 2.161406 & -2.553138 & 1.589048 \\ \mathrm{C} & 3.987615 & -3.136642 & -0.029161 \\ \mathrm{C} & 2.564915 & 0.842287 & 2.098101 \\ \mathrm{C} & 1.952142 & -0.223774 & 1.158170 \\ \mathrm{C} & 1.433696 & -1.422899 & 1.939771 \\ \mathrm{C} & 3.086421 & -2.272960 & 0.598340 \\ \mathrm{C} & 3.042404 & -0.891966 & 0.306572 \\ \mathrm{C} & 3.976169 & -0.361955 & -0.576248 \\ \mathrm{C} & 4.897998 & -1.211365 & -1.209054 \\ \mathrm{C} & 4.890081 & -2.584459 & -0.942566 \\ \mathrm{H} & 1.987209 & -3.477400 & 1.955555 \\ \mathrm{H} & 3.998785 & -4.198624 & 0.199725 \\ \mathrm{H} & 2.988301 & 1.675172 & 1.526966 \\ \mathrm{H} & 1.812886 & 1.249474 & 2.781242 \\ \mathrm{H} & 0.981400 & -1.375383 & 2.923297 \\ \mathrm{H} & 4.006007 & 0.704473 & -0.773827 \\ \mathrm{H} & 5.622632 & -0.797338 & -1.904159 \\ \mathrm{H} & 5.605121 & -3.234531 & -1.440082 \\ \mathrm{H} & 3.366367 & 0.393486 & 2.693216 \\ \mathrm{~N} & -0.323586 & 2.244481 & 1.395226 \\ \mathrm{C} & -2.246010 & -1.337030 & -1.249517 \\ \mathrm{O} & -1.880554 & -2.349385 & -1.848358 \\ \mathrm{C} & -1.285549 & -0.196774 & -0.900482 \\ \mathrm{C} & 0.460821 & 4.099452 & -0.105727\end{array}$




$\begin{array}{lrrr}\text { C } & -3.970786 & 0.064898 & -0.103156 \\ \mathrm{C} & -5.246928 & -0.427214 & 0.594132 \\ \mathrm{C} & -5.822270 & -1.437199 & -0.414569 \\ \mathrm{C} & -4.581698 & -2.129516 & -1.006346 \\ \mathrm{C} & -2.891477 & 0.596809 & 0.838490 \\ \mathrm{C} & 0.169930 & -0.632461 & -0.702492 \\ \mathrm{C} & 0.761520 & 0.398408 & 0.286776 \\ \mathrm{C} & -0.451285 & 0.808704 & 1.193984 \\ \mathrm{C} & 0.377228 & 2.768431 & 0.303338 \\ \mathrm{C} & 1.056117 & 1.743134 & -0.383417 \\ \mathrm{C} & 1.787406 & 2.053693 & -1.525603 \\ \mathrm{C} & 1.879403 & 3.387553 & -1.951231 \\ \mathrm{C} & 1.226549 & 4.396590 & -1.239091 \\ \mathrm{~N} & -1.628352 & 0.415473 & 0.393146 \\ \mathrm{~N} & -3.519912 & -1.123041 & -0.850388 \\ \mathrm{O} & -3.161443 & 1.160334 & 1.902322 \\ \mathrm{H} & -1.171656 & 2.709542 & 1.700683 \\ \mathrm{H} & -1.353797 & 0.557753 & -1.699032 \\ \mathrm{H} & -0.068178 & 4.881749 & 0.431761 \\ \mathrm{H} & -4.219052 & 0.876393 & -0.806624 \\ \mathrm{H} & -5.929909 & 0.392947 & 0.825132 \\ \mathrm{H} & -4.980990 & -0.920362 & 1.535310 \\ \mathrm{H} & -6.369630 & -0.909692 & -1.203969 \\ \mathrm{H} & -6.509071 & -2.151304 & 0.047276 \\ \mathrm{H} & -4.699387 & -2.402852 & -2.059150 \\ \mathrm{H} & -4.308225 & -3.037015 & -0.454747 \\ \mathrm{H} & 0.715499 & -0.666167 & -1.647182 \\ \mathrm{H} & 0.190473 & -1.633027 & -0.261932 \\ \mathrm{H} & -0.503294 & 0.295303 & 2.154811 \\ \mathrm{H} & 2.273418 & 1.271758 & -2.100988 \\ \mathrm{H} & 2.450444 & 3.631533 & -2.842316 \\ \mathrm{H} & 1.297264 & 5.427609 & -1.575999\end{array}$

Figure 11B Radical C4 TS

02

$\begin{array}{lrrr}\mathrm{N} & 1.620635 & -4.001210 & 0.629230 \\ \mathrm{C} & 2.220096 & -3.150934 & -1.665412 \\ \mathrm{C} & 2.447052 & -1.289044 & 3.023908 \\ \mathrm{C} & 2.137057 & -2.168932 & 1.845743 \\ \mathrm{C} & 1.707880 & -3.480784 & 1.902941 \\ \mathrm{C} & 2.012995 & -3.042757 & -0.280583 \\ \mathrm{C} & 2.316723 & -1.867312 & 0.453662 \\ \mathrm{C} & 2.715423 & -0.676594 & -0.254214 \\ \mathrm{C} & 3.075616 & -0.862760 & -1.634217 \\ \mathrm{C} & 2.780541 & -2.039245 & -2.316459 \\ \mathrm{H} & 1.370546 & -4.952881 & 0.405647 \\ \mathrm{H} & 2.006109 & -4.071093 & -2.200993 \\ \mathrm{H} & 3.527632 & -1.113459 & 3.115482 \\ \mathrm{H} & 1.973948 & -0.303156 & 2.947642 \\ \mathrm{H} & 1.467843 & -4.089735 & 2.763819 \\ \mathrm{H} & 3.247593 & 0.087841 & 0.305776 \\ \mathrm{H} & 3.527212 & -0.032211 & -2.169301 \\ \mathrm{H} & 3.008859 & -2.111203 & -3.376812 \\ \mathrm{H} & 2.110394 & -1.752856 & 3.957095 \\ \mathrm{~N} & 0.663978 & 1.696845 & 1.638795\end{array}$

$\begin{array}{rrrr}\mathrm{C} & -2.436458 & -0.261094 & -1.592535 \\ \mathrm{O} & -2.384917 & -0.960078 & -2.605491 \\ \mathrm{C} & -1.258223 & 0.596067 & -1.124085 \\ \mathrm{C} & 1.676579 & 3.913617 & 1.017803 \\ \mathrm{C} & -3.599933 & 0.641960 & 0.428299 \\ \mathrm{C} & -4.749711 & -0.011105 & 1.207509 \\ \mathrm{C} & -5.693186 & -0.491863 & 0.090445 \\ \mathrm{C} & -4.745558 & -0.964550 & -1.025845 \\ \mathrm{C} & -2.260251 & 0.672878 & 1.165038 \\ \mathrm{C} & 0.132812 & 0.067574 & -1.530985 \\ \mathrm{C} & 1.028871 & 0.596874 & -0.427276 \\ \mathrm{C} & 0.205790 & 0.554501 & 0.864929 \\ \mathrm{C} & 1.290380 & 2.597295 & 0.774909 \\ \mathrm{C} & 1.543028 & 1.971917 & -0.472553 \\ \mathrm{C} & 2.196773 & 2.685614 & -1.481079 \\ \mathrm{C} & 2.590307 & 4.007793 & -1.243653 \\ \mathrm{C} & 2.331049 & 4.610550 & -0.007039 \\ \mathrm{~N} & -1.184320 & 0.650036 & 0.348741 \\ \mathrm{~N} & -3.529995 & -0.166889 & -0.802652 \\ \mathrm{O} & -2.179521 & 0.734392 & 2.394643 \\ \mathrm{H} & 0.040445 & 2.030865 & 2.364086 \\ \mathrm{H} & -1.399122 & 1.611528 & -1.525046 \\ \mathrm{H} & 1.468278 & 4.390115 & 1.971855 \\ \mathrm{H} & -3.861415 & 1.682854 & 0.176904 \\ \mathrm{H} & -5.220877 & 0.685999 & 1.903698 \\ \mathrm{H} & -4.363263 & -0.856925 & 1.786016 \\ \mathrm{H} & -6.307268 & 0.342344 & -0.267512 \\ \mathrm{H} & -6.368471 & -1.286259 & 0.419016 \\ \mathrm{H} & -5.138578 & -0.790389 & -2.031914 \\ \mathrm{H} & -4.503945 & -2.031053 & -0.941975 \\ \mathrm{H} & 0.420460 & 0.414935 & -2.524714 \\ \mathrm{H} & 0.121289 & -1.026742 & -1.546593 \\ \mathrm{H} & 0.284900 & -0.365873 & 1.447391 \\ \mathrm{H} & 2.386169 & 2.223722 & -2.446689 \\ \mathrm{H} & 3.094306 & 4.570155 & -2.024547 \\ \mathrm{H} & 2.636453 & 5.639491 & 0.164146\end{array}$

Figure 11B Radical C4 Product 02

$\begin{array}{lrrr}\mathrm{N} & 2.339850 & -3.871873 & 0.285473 \\ \mathrm{C} & 3.077094 & -2.534350 & -1.705250 \\ \mathrm{C} & 1.876747 & -1.511342 & 3.099210 \\ \mathrm{C} & 2.065900 & -2.205459 & 1.774932 \\ \mathrm{C} & 2.066670 & -3.585487 & 1.599105 \\ \mathrm{C} & 2.554862 & -2.696746 & -0.403707 \\ \mathrm{C} & 2.343935 & -1.633887 & 0.498713 \\ \mathrm{C} & 2.486395 & -0.203790 & 0.034136 \\ \mathrm{C} & 3.299653 & -0.143072 & -1.243021 \\ \mathrm{C} & 3.492699 & -1.226772 & -2.062895 \\ \mathrm{H} & 2.473944 & -4.798775 & -0.091312 \\ \mathrm{H} & 3.249502 & -3.382704 & -2.359777 \\ \mathrm{H} & 0.830039 & -1.503755 & 3.430702 \\ \mathrm{H} & 2.454888 & -2.008916 & 3.886351 \\ \mathrm{H} & 1.899658 & -4.375727 & 2.317741 \\ \mathrm{H} & 2.985331 & 0.390146 & 0.814203\end{array}$




$\begin{array}{rrrr}\mathrm{H} & 3.673344 & 0.829744 & -1.545461 \\ \mathrm{H} & 4.011680 & -1.088257 & -3.008901 \\ \mathrm{H} & 2.207308 & -0.468982 & 3.054755 \\ \mathrm{~N} & 0.418714 & 1.710266 & 1.767619 \\ \mathrm{C} & -2.242272 & -0.541080 & -1.636418 \\ \mathrm{O} & -2.064050 & -1.250178 & -2.627615 \\ \mathrm{C} & -1.165280 & 0.404467 & -1.096929 \\ \mathrm{C} & 0.989047 & 4.055466 & 1.055934 \\ \mathrm{C} & -3.617383 & 0.310075 & 0.269551 \\ \mathrm{C} & -4.774272 & -0.410326 & 0.975202 \\ \mathrm{C} & -5.594185 & -0.976580 & -0.197936 \\ \mathrm{C} & -4.533720 & -1.398127 & -1.230293 \\ \mathrm{C} & -2.343792 & 0.447253 & 1.103456 \\ \mathrm{C} & 0.273889 & -0.042680 & -1.378492 \\ \mathrm{C} & 1.096526 & 0.555259 & -0.218894 \\ \mathrm{C} & 0.129640 & 0.508015 & 1.003518 \\ \mathrm{C} & 0.889023 & 2.677067 & 0.869209 \\ \mathrm{C} & 1.308950 & 2.066162 & -0.327968 \\ \mathrm{C} & 1.815159 & 2.848950 & -1.359884 \\ \mathrm{C} & 1.918767 & 4.237845 & -1.188042 \\ \mathrm{C} & 1.512881 & 4.827289 & 0.011783 \\ \mathrm{~N} & -1.208670 & 0.489864 & 0.372104 \\ \mathrm{~N} & -3.397548 & -0.511597 & -0.934640 \\ \mathrm{O} & -2.362333 & 0.529836 & 2.334625 \\ \mathrm{H} & -0.297051 & 1.972439 & 2.436927 \\ \mathrm{H} & -1.344590 & 1.399831 & -1.531387 \\ \mathrm{H} & 0.657930 & 4.518922 & 1.981252 \\ \mathrm{H} & -3.931051 & 1.325376 & -0.023322 \\ \mathrm{H} & -5.344458 & 0.262901 & 1.618843 \\ \mathrm{H} & -4.374286 & -1.216397 & 1.599465 \\ \mathrm{H} & -6.237171 & -0.195378 & -0.619114 \\ \mathrm{H} & -6.234299 & -1.812055 & 0.097488 \\ \mathrm{H} & -4.861523 & -1.268733 & -2.266021 \\ \mathrm{H} & -4.224360 & -2.442953 & -1.106223 \\ \mathrm{H} & 0.618807 & 0.295770 & -2.356543 \\ \mathrm{H} & 0.332821 & -1.134743 & -1.362420 \\ \mathrm{H} & 0.219781 & -0.375793 & 1.629515 \\ \mathrm{H} & 2.115236 & 2.397240 & -2.301419 \\ \mathrm{H} & 2.309495 & 4.853914 & -1.992683 \\ \mathrm{H} & 1.592068 & 5.904108 & 0.137229\end{array}$

Figure 11B Radical C5 TS 02
N $\quad-5.566010$
$0.067828-0.750328$
C -3.454605
$\begin{array}{lll}0.700912 & -1.990373\end{array}$
C -4.832501
$\begin{array}{ll}-3.102094 & 1.047864\end{array}$
C -4.862720
$-1.840573$
0.238460
C -5.927209
$-0.991043$
0.067570
C -4.252694
$-0.077585-1.125277$
C -3.766090
$-1.271044-0.515001$
$-2.448732$
$-1.672261$
$-0.752188$
$-1.566897$
$-0.813989$
$-1.490162$
C -2.154795
0.306253
0.822785
$-2.202178$
H -6.176279
H -3.867343
$\begin{array}{ll}1.568247 & -2.499427\end{array}$

$\begin{array}{rrrr}\mathrm{H} & -4.064844 & -3.062957 & 1.832136 \\ \mathrm{H} & -5.796425 & -3.281852 & 1.535252 \\ \mathrm{H} & -6.929326 & -1.051219 & 0.470160 \\ \mathrm{H} & -2.068121 & -2.601321 & -0.336045 \\ \mathrm{H} & -0.719156 & -1.296211 & -1.977442 \\ \mathrm{H} & -1.526915 & 0.877519 & -2.879386 \\ \mathrm{H} & -4.605915 & -3.979222 & 0.427063 \\ \mathrm{~N} & 0.370239 & 2.191987 & -0.782233 \\ \mathrm{C} & 3.033387 & -1.588206 & 0.786701 \\ \mathrm{O} & 2.865040 & -2.775631 & 1.068131 \\ \mathrm{C} & 1.886553 & -0.574303 & 0.794455 \\ \mathrm{C} & -0.928421 & 3.435362 & 0.975946 \\ \mathrm{C} & 4.450511 & 0.349733 & 0.084470 \\ \mathrm{C} & 5.747855 & 0.295457 & -0.733970 \\ \mathrm{C} & 6.529909 & -0.847469 & -0.062876 \\ \mathrm{C} & 5.448422 & -1.877127 & 0.310084 \\ \mathrm{C} & 3.250176 & 0.937567 & -0.657262 \\ \mathrm{C} & 0.495758 & -1.170169 & 0.492859 \\ \mathrm{C} & -0.249851 & -0.002293 & -0.133561 \\ \mathrm{C} & 0.783946 & 0.807292 & -0.931227 \\ \mathrm{C} & -0.479647 & 2.290720 & 0.321110 \\ \mathrm{C} & -0.896340 & 0.998166 & 0.725834 \\ \mathrm{C} & -1.777322 & 0.862332 & 1.800922 \\ \mathrm{C} & -2.236110 & 2.008060 & 2.462871 \\ \mathrm{C} & -1.813846 & 3.277280 & 2.051555 \\ \mathrm{~N} & 2.057237 & 0.441918 & -0.260771 \\ \mathrm{~N} & 4.233375 & -1.060095 & 0.456805 \\ \mathrm{O} & 3.369234 & 1.804301 & -1.526856 \\ \mathrm{H} & 1.075567 & 2.898458 & -0.953090 \\ \mathrm{H} & 1.873819 & -0.086820 & 1.781145 \\ \mathrm{H} & -0.594050 & 4.422834 & 0.669273 \\ \mathrm{H} & 4.605929 & 0.956180 & 0.991746 \\ \mathrm{H} & 6.278617 & 1.249778 & -0.724339 \\ \mathrm{H} & 5.511617 & 0.050567 & -1.775019 \\ \mathrm{H} & 7.026897 & -0.481508 & 0.842661 \\ \mathrm{H} & 7.296375 & -1.275343 & -0.714376 \\ \mathrm{H} & 5.663680 & -2.414495 & 1.238490 \\ \mathrm{H} & 5.293505 & -2.623200 & -0.478554 \\ \mathrm{H} & 0.015751 & -1.549716 & 1.396973 \\ \mathrm{H} & 0.606425 & -2.008434 & -0.205451 \\ \mathrm{H} & 0.881116 & 0.552887 & -1.991896 \\ \mathrm{H} & -2.101273 & -0.123659 & 2.122621 \\ \mathrm{H} & -2.918971 & 1.910454 & 3.302204 \\ \mathrm{H} & -2.171186 & 4.159557 & 2.576477\end{array}$

Figure 11B Radical C5 Product 02

$\begin{array}{lrrr}\text { N } & -5.707588 & -0.106556 & -0.984684 \\ C & -3.514493 & 0.747008 & -1.934977 \\ \text { C } & -4.888469 & -3.070003 & 1.101239 \\ \text { C } & -4.944779 & -1.862661 & 0.215120 \\ \text { C } & -6.064454 & -1.160100 & -0.159222 \\ \text { C } & -4.344925 & -0.109478 & -1.157341 \\ \text { C } & -3.817546 & -1.201966 & -0.414240 \\ \text { C } & -2.448342 & -1.453522 & -0.410841\end{array}$




\begin{tabular}{|c|c|c|c|}
\hline & & & \\
\hline & 12 & & \\
\hline & 730 & & \\
\hline & & & \\
\hline & 773 & & \\
\hline & 7756 & 22 & \\
\hline & 804 & & \\
\hline & 811 & & \\
\hline & 361 & & \\
\hline & -1 . & & \\
\hline & -4.4 & -3.5 & \\
\hline & & & \\
\hline & & & \\
\hline & 2.7 & -2.7 & \\
\hline & 1.82 & -0.5 & \\
\hline & & & \\
\hline & & & \\
\hline & & & \\
\hline & & & \\
\hline & & -1. & \\
\hline & 3.22 & & \\
\hline & & & \\
\hline & & & \\
\hline & & & \\
\hline & & & \\
\hline & & & \\
\hline & & & \\
\hline & & & \\
\hline & -1.4 & & \\
\hline & & & \\
\hline & & & \\
\hline & & & \\
\hline & 1.2 & & \\
\hline & & & \\
\hline & -0.1 & & \\
\hline & & & \\
\hline & & & \\
\hline & 5.4 & -0.2 & \\
\hline & & & \\
\hline & & & \\
\hline & & & \\
\hline & & & \\
\hline & -0.049433 & & \\
\hline & & & \\
\hline & 0.816040 & & \\
\hline & -2.038898 & & \\
\hline & -2.571343 & & \\
\hline & -1.647894 & 4.307559 & 2.721 \\
\hline
\end{tabular}

Figure 11B Radical C6 TS

02
$\begin{array}{llll}\text { N } & 2.477549 & -3.282471 & -1.302257\end{array}$
$\begin{array}{llll}\text { C } & 2.166737 & -0.851400 & -1.866568\end{array}$
$\begin{array}{llll}\text { C } & 4.390644 & -3.390378 & 1.883507\end{array}$
$\begin{array}{llll}\text { C } & 3.633059 & -3.060107 & 0.631191\end{array}$

\begin{tabular}{|c|c|c|c|}
\hline & & & \\
\hline & 307 & & \\
\hline & & & \\
\hline & 7505 & & \\
\hline & 071 & 9 & \\
\hline & 196 & 5059 & \\
\hline & 614 & -3.716455 & \\
\hline & 1.6 & 1808 & \\
\hline & 3.8 & 2834 & \\
\hline & 4.48 & 3549 & \\
\hline & 3.11 & -5.039917 & -0.2 \\
\hline & & -0.278233 & \\
\hline & & & \\
\hline & & & \\
\hline & 5.4 & & \\
\hline & & & \\
\hline & & & \\
\hline & & & \\
\hline & & & \\
\hline & & & \\
\hline & & & \\
\hline & & & \\
\hline & -5 & & \\
\hline & -4.6 & $-1 . \varsigma$ & -0 . \\
\hline & & -0 & \\
\hline & & & \\
\hline & & & \\
\hline & & & \\
\hline & & & \\
\hline & & & \\
\hline & & & \\
\hline & & & \\
\hline & & & \\
\hline & & & \\
\hline & & -0 & -0. \\
\hline & & & \\
\hline & & & \\
\hline & -2 & & \\
\hline & & & \\
\hline & & & \\
\hline & & & \\
\hline & -3.4 & -2.5 & \\
\hline & -6.1 & -1.5 & \\
\hline & -5.6 & -3.1821 & \\
\hline & -5.35 & -1.570319 & -1.51324 \\
\hline & -4.1 & -2.795669 & -1.13295 \\
\hline & -0.6 & 1.516232 & -2.56577 \\
\hline & -0.3 & -0.181050 & -2.19097 \\
\hline & & -0.4064 & \\
\hline & & & -2.28255 \\
\hline & & 5.890243 & -1.22473 \\
\hline & & 010 & 18 \\
\hline
\end{tabular}

Figure 11B Radical C6 Product 02 


\begin{tabular}{|c|c|c|c|}
\hline & & & \\
\hline & & & \\
\hline & & 360 & \\
\hline & 7764 & 450 & \\
\hline & 976 & 054 & . \\
\hline & 943116 & 385 & -0 \\
\hline & 30748 & 297 & \\
\hline & 2760 & 500 & \\
\hline & & 375 & \\
\hline & & & \\
\hline & & & \\
\hline & & & \\
\hline & & & \\
\hline & & & \\
\hline & & & \\
\hline & & & \\
\hline & & & \\
\hline & & & \\
\hline & & & \\
\hline & & & \\
\hline & & & \\
\hline & & & \\
\hline & & & \\
\hline & & & \\
\hline & & & \\
\hline & & & \\
\hline & & & \\
\hline & -4.7 & & \\
\hline & & & \\
\hline & -0.6 & & \\
\hline & & & \\
\hline & & & \\
\hline & & & \\
\hline$\checkmark$ & & & \\
\hline 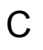 & 0.3 & & \\
\hline ; & & & \\
\hline 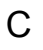 & & & \\
\hline$N$ & & & \\
\hline T & -3.7 & & \\
\hline 0 & & & \\
\hline 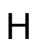 & -0.2 & & \\
\hline-1 & & & \\
\hline-1 & & & \\
\hline $\mathrm{H}$ & & & \\
\hline $\mathrm{H}$ & & & \\
\hline 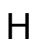 & & & \\
\hline $\mathrm{H}$ & & & \\
\hline $\mathrm{H}$ & -5.6 & & \\
\hline $\mathrm{H}$ & & & \\
\hline $\mathrm{H}$ & & & \\
\hline $\mathrm{H}$ & & & \\
\hline & & & \\
\hline & & & \\
\hline & & & \\
\hline & & & \\
\hline
\end{tabular}

$\begin{array}{llll}H & 0.048921 & 6.003156 & 1.108717\end{array}$

Figure 11B Radical C7 TS

02

$\begin{array}{llll}\mathrm{N} & -2.892314 & 0.454058 & -1.848977\end{array}$

C $\quad-1.664756 \quad-1.556181 \quad-0.917054$

$\begin{array}{llll}\text { C } & -6.416100 & 0.240288 & -0.687096\end{array}$

$\begin{array}{llll}\text { C } & -4.957892 & 0.094171 & -1.006261\end{array}$

C $\quad-4.180383 \quad 0.954574 \quad-1.741089$

$\begin{array}{llll}\text { C } & -2.810016 & -0.723807 & -1.148817\end{array}$

C $\quad-4.089200 \quad-0.987566-0.606192$

$\begin{array}{llll}\text { C } & -4.288594 & -2.157098 & 0.159709\end{array}$

$\begin{array}{llll}\text { C } & -3.206635 & -3.024440 & 0.353660\end{array}$

$\begin{array}{llll}\text { C } & -1.953171 & -2.766865 & -0.203632\end{array}$

$\begin{array}{llll}\mathrm{H} & -2.086921 & 1.003447 & -2.124869\end{array}$

H $\quad-0.893341 \quad-1.602786 \quad-1.684137$

H $\quad-6.595894 \quad 0.242032 \quad 0.395973$

H $\quad-6.815600 \quad 1.176655 \quad-1.090529$

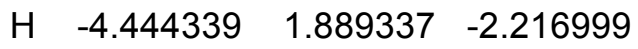

H $\quad-5.263719 \quad-2.387556 \quad 0.579917$

H $\quad-3.353924 \quad-3.941201 \quad 0.919202$

H $\quad-1.158631 \quad-3.497593 \quad-0.083118$

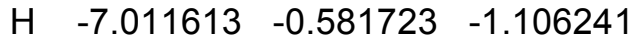

$\begin{array}{llll}\mathrm{N} & -0.172638 & 1.747898 & -0.744439\end{array}$

$\begin{array}{llll}\text { C } & 3.321457 & -1.319006 & 0.793663\end{array}$

$\begin{array}{llll}\text { O } & 3.410660 & -2.493549 & 1.153293\end{array}$

$\begin{array}{llll}\text { C } & 2.014226 & -0.528146 & 0.893300\end{array}$

$\begin{array}{llll}\text { C } & -1.427957 & 2.960829 & 1.072083\end{array}$

C $\quad 4.256405 \quad 0.787461 \quad-0.176567$

C $5.452465 \quad 0.920950-1.129165$

$\begin{array}{llll}\text { C } & 6.501724 & -0.010902 & -0.497375\end{array}$

$\begin{array}{llll}\text { C } & 5.679816 & -1.196780 & 0.036970\end{array}$

$\begin{array}{llll}\text { C } & 2.895953 & 1.076692 & -0.810771\end{array}$

$\begin{array}{llll}\text { C } & 0.731562 & -1.384358 & 0.812287\end{array}$

$\begin{array}{llll}\text { C } & -0.277104 & -0.419688 & 0.217891\end{array}$

C $\quad 0.491782 \quad 0.451433 \quad-0.785599$

$\begin{array}{llll}\text { C } & -0.864157 & 1.837437 & 0.475521\end{array}$

C $\quad-0.974520 \quad 0.553459 \quad 1.063036$

C $\quad-1.677332 \quad 0.400940 \quad 2.261425$

C $\quad-2.250963 \quad 1.524802 \quad 2.866136$

$\begin{array}{llll}\text { C } & -2.123897 & 2.788270 & 2.276763\end{array}$

$\begin{array}{llll}\mathrm{N} & 1.868063 & 0.413577 & -0.234477\end{array}$

$\begin{array}{llll}N & 4.355297 & -0.607980 & 0.290270\end{array}$

$\begin{array}{llll}\mathrm{O} & 2.752416 & 1.860843 & -1.751761\end{array}$

$\begin{array}{llll}\mathrm{H} & 0.389294 & 2.533510 & -1.054504\end{array}$

$\begin{array}{llll}\mathrm{H} & 2.026187 & 0.029442 & 1.841697\end{array}$

$\begin{array}{llll}\mathrm{H} & -1.327223 & 3.944636 & 0.621803\end{array}$

$\begin{array}{llll}\mathrm{H} & 4.376808 & 1.476154 & 0.675490\end{array}$

H $\quad 5.789500 \quad 1.955636 \quad-1.220978$

$\mathrm{H} \quad 5.164855 \quad 0.569692 \quad-2.125814$

H $\quad \begin{array}{llll}7.007172 & 0.498220 & 0.331084\end{array}$

H $\quad \begin{array}{llll}7.267033 & -0.330041 & -1.209671\end{array}$

H $\quad 6.087935 \quad-1.629386 \quad 0.955182$

H $\quad 5.586588-2.003715 \quad-0.699815$

H $\quad 0.437285 \quad-1.767581 \quad 1.791111$ 


$\begin{array}{rrrr}H & 0.912609 & -2.242642 & 0.155853 \\ H & 0.533996 & 0.083953 & -1.816376 \\ H & -1.773466 & -0.579632 & 2.720230 \\ H & -2.795332 & 1.416949 & 3.799954 \\ H & -2.568985 & 3.654157 & 2.759705\end{array}$

Figure 11B Radical C7 Product

$\begin{array}{lrrr}02 & & & \\ \mathrm{~N} & -2.749404 & 0.588212 & -1.668534 \\ \mathrm{C} & -1.565597 & -1.344318 & -0.448539 \\ \mathrm{C} & -6.417170 & 0.076139 & -1.457907 \\ \mathrm{C} & -4.918690 & 0.058967 & -1.384589 \\ \mathrm{C} & -4.052147 & 0.974734 & -1.932147 \\ \mathrm{C} & -2.758327 & -0.553932 & -0.912675 \\ \mathrm{C} & -4.097646 & -0.925722 & -0.723321 \\ \mathrm{C} & -4.424894 & -2.085244 & 0.034263 \\ \mathrm{C} & -3.365106 & -2.827857 & 0.596351 \\ \mathrm{C} & -2.041640 & -2.496403 & 0.412502 \\ \mathrm{H} & -1.936926 & 1.194460 & -1.762016 \\ \mathrm{H} & -1.064385 & -1.785654 & -1.336005 \\ \mathrm{H} & -6.874014 & 0.097266 & -0.459625 \\ \mathrm{H} & -6.773922 & 0.956491 & -2.003054 \\ \mathrm{H} & -4.253869 & 1.876710 & -2.493605 \\ \mathrm{H} & -5.457615 & -2.378185 & 0.195308 \\ \mathrm{H} & -3.599647 & -3.704781 & 1.196455 \\ \mathrm{H} & -1.275981 & -3.136302 & 0.839368 \\ \mathrm{H} & -6.813512 & -0.811619 & -1.968691 \\ \mathrm{~N} & -0.221474 & 1.738705 & -0.636559 \\ \mathrm{C} & 3.265009 & -1.345483 & 0.722345 \\ \mathrm{O} & 3.375754 & -2.501592 & 1.131233 \\ \mathrm{C} & 1.955479 & -0.559142 & 0.829930 \\ \mathrm{C} & -1.122259 & 2.970459 & 1.371492 \\ \mathrm{C} & 4.148882 & 0.722686 & -0.370206 \\ \mathrm{C} & 5.299978 & 0.817075 & -1.381510 \\ \mathrm{C} & 6.381296 & -0.080969 & -0.754566 \\ \mathrm{C} & 5.591017 & -1.243769 & -0.129188 \\ \mathrm{C} & 2.761873 & 0.995064 & -0.949245 \\ \mathrm{C} & 0.692749 & -1.423211 & 0.815087 \\ \mathrm{C} & -0.410198 & -0.503686 & 0.234456 \\ \mathrm{C} & 0.364911 & 0.407509 & -0.780747 \\ \mathrm{C} & -0.742152 & 1.826195 & 0.673436 \\ \mathrm{C} & -0.914196 & 0.543540 & 1.218969 \\ \mathrm{C} & -1.487118 & 0.394305 & 2.476986 \\ \mathrm{C} & -1.874960 & 1.536094 & 3.192069 \\ \mathrm{C} & -1.688762 & 2.807414 & 2.641416 \\ \mathrm{~N} & 1.763706 & 0.321535 & -0.331895 \\ \mathrm{~N} & 4.273137 & -0.651105 & 0.149561 \\ \mathrm{O} & 2.572698 & 1.778927 & -1.881969 \\ \mathrm{H} & 0.378404 & 2.489460 & -0.965938 \\ \mathrm{H} & -1.994204 & 0.040806 & 1.751782 \\ & 4.308075 & 3.959768 & 0.944851 \\ \mathrm{H} & 5.626227 & 1.446651 & 0.445510 \\ & 1.847740 & -1.535312 \\ & & \\ \mathrm{H} & & \end{array}$

$\begin{array}{rrrr}\mathrm{H} & 7.115785 & -0.430237 & -1.484844 \\ \mathrm{H} & 6.040158 & -1.627900 & 0.791395 \\ \mathrm{H} & 5.473071 & -2.086643 & -0.820606 \\ \mathrm{H} & 0.447227 & -1.796289 & 1.810569 \\ \mathrm{H} & 0.853750 & -2.287814 & 0.162045 \\ \mathrm{H} & 0.309191 & 0.082203 & -1.823156 \\ \mathrm{H} & -1.632271 & -0.594540 & 2.903601 \\ \mathrm{H} & -2.318336 & 1.431485 & 4.177981 \\ \mathrm{H} & -1.986750 & 3.687377 & 3.205323\end{array}$

Figure 11D Cation C2 TS

$\begin{array}{lrrr}11 & & & \\ \mathrm{~N} & 1.501309 & -1.347979 & 1.780037 \\ \mathrm{C} & 1.512447 & -3.761497 & 1.061592 \\ \mathrm{C} & 4.287954 & 0.271535 & -0.064632 \\ \mathrm{C} & 3.196383 & -0.595981 & 0.464102 \\ \mathrm{C} & 2.200847 & -0.213198 & 1.396802 \\ \mathrm{C} & 1.941693 & -2.427959 & 1.053326 \\ \mathrm{C} & 3.001857 & -1.978597 & 0.206975 \\ \mathrm{C} & 3.645507 & -2.903280 & -0.649772 \\ \mathrm{C} & 3.225392 & -4.218768 & -0.636694 \\ \mathrm{C} & 2.169474 & -4.639827 & 0.212388 \\ \mathrm{H} & 0.806079 & -1.386188 & 2.511643 \\ \mathrm{H} & 0.705626 & -4.093279 & 1.707043 \\ \mathrm{H} & 4.479147 & 0.083232 & -1.126610 \\ \mathrm{H} & 4.069154 & 1.332748 & 0.072551 \\ \mathrm{H} & 2.228907 & 0.648161 & 2.050799 \\ \mathrm{H} & 4.455409 & -2.582645 & -1.297907 \\ \mathrm{H} & 3.704572 & -4.947439 & -1.282749 \\ \mathrm{H} & 1.866686 & -5.682568 & 0.195750 \\ \mathrm{H} & 5.222711 & 0.059943 & 0.472086 \\ \mathrm{~N} & -0.140728 & 2.607873 & 1.308266 \\ \mathrm{C} & -2.153191 & -0.987562 & -1.305574 \\ \mathrm{O} & -1.758441 & -1.992945 & -1.894588 \\ \mathrm{C} & -1.231037 & 0.212866 & -1.058207 \\ \mathrm{C} & 1.109938 & 4.511145 & 0.289322 \\ \mathrm{C} & -3.868491 & 0.359024 & -0.080314 \\ \mathrm{C} & -5.094182 & -0.163580 & 0.681296 \\ \mathrm{C} & -5.669498 & -1.221125 & -0.277595 \\ \mathrm{C} & -4.428616 & -1.884146 & -0.898948 \\ \mathrm{C} & -2.760705 & 0.929862 & 0.801917 \\ \mathrm{C} & 0.268358 & -0.165997 & -0.955231 \\ \mathrm{C} & 0.772445 & 0.886741 & -0.007661 \\ \mathrm{C} & -0.330496 & 1.203187 & 1.016424 \\ \mathrm{C} & 0.755344 & 3.162176 & 0.450316 \\ \mathrm{C} & 1.323677 & 2.145385 & -0.387144 \\ \mathrm{C} & 2.200913 & 2.509504 & -1.435413 \\ \mathrm{C} & 2.533155 & 3.840359 & -1.598411 \\ \mathrm{C} & 1.997368 & 4.823959 & -0.729361 \\ \mathrm{~N} & -1.522401 & 0.861332 & 0.238639 \\ \mathrm{~N} & -3.404759 & -0.826399 & -0.827838 \\ \mathrm{O} & -2.951750 & 1.420239 & 1.910785 \\ \mathrm{H} & -0.731847 & 3.107768 & 1.956862 \\ \mathrm{H} & -1.373910 & 0.933694 & -1.875078 \\ \mathrm{H} & 0.686293 & 5.280500 & 0.926062\end{array}$




$\begin{array}{rrrr}\mathrm{H} & -4.169516 & 1.151529 & -0.783799 \\ \mathrm{H} & -5.798755 & 0.636247 & 0.917963 \\ \mathrm{H} & -4.771723 & -0.620473 & 1.622696 \\ \mathrm{H} & -6.266515 & -0.736990 & -1.058230 \\ \mathrm{H} & -6.309295 & -1.946177 & 0.231333 \\ \mathrm{H} & -4.576814 & -2.199618 & -1.935332 \\ \mathrm{H} & -4.090653 & -2.755727 & -0.326372 \\ \mathrm{H} & 0.764122 & -0.141847 & -1.925878 \\ \mathrm{H} & 0.354804 & -1.176941 & -0.547304 \\ \mathrm{H} & -0.333060 & 0.632262 & 1.946815 \\ \mathrm{H} & 2.603795 & 1.751569 & -2.099368 \\ \mathrm{H} & 3.204606 & 4.142205 & -2.395050 \\ \mathrm{H} & 2.278544 & 5.862536 & -0.878243\end{array}$

Figure 11D Cation C2 Product

11

N

c

c

c

c c

c c

\section{c}

c

\section{(a)}

do

\section{(a)}

c
$1.951542-1.0$

$3.289477-3.1$

$$
3.855563
$$$$
3.253263
$$$$
1.995487
$$$$
2.967209
$$$$
3.799507
$$$$
-1.052607
$$

$\begin{array}{rrrr}\mathrm{O} & -3.106889 & 0.385451 & 2.193067 \\ \mathrm{H} & -1.377775 & 2.262593 & 2.277487 \\ \mathrm{H} & -1.681928 & 0.735331 & -1.633339 \\ \mathrm{H} & -0.917946 & 4.818523 & 1.370274 \\ \mathrm{H} & -4.400640 & 0.358171 & -0.463149 \\ \mathrm{H} & -5.844392 & -0.662356 & 1.177857 \\ \mathrm{H} & -4.615570 & -1.855097 & 1.622223 \\ \mathrm{H} & -6.226196 & -1.739943 & -0.990753 \\ \mathrm{H} & -6.032391 & -3.140595 & 0.071087 \\ \mathrm{H} & -4.402066 & -2.777387 & -2.157969 \\ \mathrm{H} & -3.756871 & -3.515825 & -0.684401 \\ \mathrm{H} & 0.571278 & -0.005307 & -1.957886 \\ \mathrm{H} & 0.410389 & -1.278995 & -0.745058 \\ \mathrm{H} & -0.367159 & -0.014679 & 2.035004 \\ \mathrm{H} & 1.487398 & 2.312693 & -2.223039 \\ \mathrm{H} & 1.109249 & 4.750536 & -2.439409 \\ \mathrm{H} & -0.063710 & 5.997519 & -0.645747\end{array}$

Figure 11D Cation C3 TS

11

$\begin{array}{lrrr}\mathrm{N} & 1.502823 & -2.495214 & 1.567613 \\ \mathrm{C} & 3.068634 & -3.503374 & -0.146769 \\ \mathrm{C} & 2.707532 & 0.904036 & 2.192691 \\ \mathrm{C} & 2.222280 & -0.346666 & 1.511318 \\ \mathrm{C} & 1.341861 & -1.280837 & 2.112585 \\ \mathrm{C} & 2.542659 & -2.472284 & 0.627468 \\ \mathrm{C} & 3.039807 & -1.150849 & 0.597189 \\ \mathrm{C} & 4.144184 & -0.859441 & -0.211850 \\ \mathrm{C} & 4.689199 & -1.883313 & -0.987658 \\ \mathrm{C} & 4.154196 & -3.183201 & -0.961960 \\ \mathrm{H} & 0.944719 & -3.308828 & 1.799336 \\ \mathrm{H} & 2.662955 & -4.509379 & -0.111606 \\ \mathrm{H} & 3.020163 & 1.657160 & 1.462723 \\ \mathrm{H} & 1.939059 & 1.344219 & 2.834156 \\ \mathrm{H} & 0.610398 & -1.118461 & 2.892657 \\ \mathrm{H} & 4.576110 & 0.136074 & -0.230072 \\ \mathrm{H} & 5.547870 & -1.675543 & -1.618789 \\ \mathrm{H} & 4.600793 & -3.956820 & -1.578742 \\ \mathrm{H} & 3.577475 & 0.671487 & 2.817738 \\ \mathrm{~N} & -0.204073 & 2.508775 & 1.000737 \\ \mathrm{C} & -2.358858 & -1.329471 & -1.080285 \\ \mathrm{O} & -2.016551 & -2.455754 & -1.439328 \\ \mathrm{C} & -1.357661 & -0.170491 & -0.984121 \\ \mathrm{C} & 1.219011 & 4.097056 & -0.298961 \\ \mathrm{C} & -4.016307 & 0.346984 & -0.250625 \\ \mathrm{C} & -5.336727 & 0.073783 & 0.483187 \\ \mathrm{C} & -5.939725 & -1.095504 & -0.315749 \\ \mathrm{C} & -4.725739 & -1.959466 & -0.694398 \\ \mathrm{C} & -2.920175 & 0.973751 & 0.609427 \\ \mathrm{C} & 0.100312 & -0.630703 & -0.740406 \\ \mathrm{C} & 0.644988 & 0.507298 & 0.086560 \\ \mathrm{C} & -0.487154 & 1.087385 & 0.957775 \\ \mathrm{C} & 0.765579 & 2.830294 & 0.100673 \\ \mathrm{C} & 1.308391 & 1.640656 & -0.482282 \\ \mathrm{C} & 2.279113 & 1.735060 & -1.503687\end{array}$




$\begin{array}{rrrr}\mathrm{C} & 2.718834 & 2.986690 & -1.896098 \\ \mathrm{C} & 2.193555 & 4.151028 & -1.286227 \\ \mathrm{~N} & -1.660614 & 0.695274 & 0.174425 \\ \mathrm{~N} & -3.618441 & -0.988527 & -0.738477 \\ \mathrm{O} & -3.138966 & 1.674139 & 1.593820 \\ \mathrm{H} & -0.841471 & 3.160367 & 1.436087 \\ \mathrm{H} & -1.412284 & 0.414715 & -1.912563 \\ \mathrm{H} & 0.809820 & 5.000948 & 0.139923 \\ \mathrm{H} & -4.189386 & 1.020985 & -1.104638 \\ \mathrm{H} & -5.978187 & 0.956950 & 0.510131 \\ \mathrm{H} & -5.125756 & -0.222552 & 1.515995 \\ \mathrm{H} & -6.429764 & -0.720306 & -1.220909 \\ \mathrm{H} & -6.680648 & -1.658886 & 0.256790 \\ \mathrm{H} & -4.830470 & -2.458985 & -1.661372 \\ \mathrm{H} & -4.508813 & -2.725696 & 0.059239 \\ \mathrm{H} & 0.642069 & -0.767069 & -1.676528 \\ \mathrm{H} & 0.085056 & -1.586983 & -0.213586 \\ \mathrm{H} & -0.587057 & 0.703866 & 1.973944 \\ \mathrm{H} & 2.669789 & 0.837138 & -1.970413 \\ \mathrm{H} & 3.463090 & 3.083547 & -2.679336 \\ \mathrm{H} & 2.554010 & 5.122288 & -1.612959\end{array}$

Figure 11D Cation C3 Product

11

$\begin{array}{lrrr}\text { N } & 2.179513 & -2.543992 & 1.473199 \\ \mathrm{C} & 4.140154 & -3.043660 & -0.036436 \\ \mathrm{C} & 2.470277 & 0.742183 & 2.181264 \\ \mathrm{C} & 1.939014 & -0.265376 & 1.120859 \\ \mathrm{C} & 1.478722 & -1.510343 & 1.825871 \\ \mathrm{C} & 3.184610 & -2.209827 & 0.526538 \\ \mathrm{C} & 3.086601 & -0.832676 & 0.287164 \\ \mathrm{C} & 4.043078 & -0.238789 & -0.530320 \\ \mathrm{C} & 5.020347 & -1.052677 & -1.118891 \\ \mathrm{C} & 5.064564 & -2.432959 & -0.886717 \\ \mathrm{H} & 2.035935 & -3.482754 & 1.842409 \\ \mathrm{H} & 4.175552 & -4.106343 & 0.178381 \\ \mathrm{H} & 2.852917 & 1.623539 & 1.660691 \\ \mathrm{H} & 1.679053 & 1.060250 & 2.862449 \\ \mathrm{H} & 0.678934 & -1.602073 & 2.550422 \\ \mathrm{H} & 4.047535 & 0.829844 & -0.707406 \\ \mathrm{H} & 5.765866 & -0.598673 & -1.764054 \\ \mathrm{H} & 5.834689 & -3.034672 & -1.357836 \\ \mathrm{H} & 3.285869 & 0.297531 & 2.757521 \\ \mathrm{~N} & -0.302737 & 2.197058 & 1.410000 \\ \mathrm{C} & -2.307586 & -1.351369 & -1.230663 \\ \mathrm{O} & -1.946534 & -2.382760 & -1.798233 \\ \mathrm{C} & -1.329188 & -0.217033 & -0.904300 \\ \mathrm{C} & 0.509883 & 4.042366 & -0.090040 \\ \mathrm{C} & -4.002037 & 0.102914 & -0.107344 \\ \mathrm{C} & -5.299059 & -0.345969 & 0.580704 \\ \mathrm{C} & -5.888891 & -1.354118 & -0.421414 \\ \mathrm{C} & -4.661092 & -2.090354 & -0.984429 \\ \mathrm{C} & -2.915527 & 0.607572 & 0.839979 \\ \mathrm{C} & 0.118571 & -0.682850 & -0.716630 \\ \mathrm{C} & 0.723236 & 0.339994 & 0.275239\end{array}$

$\begin{array}{rrrr}\mathrm{C} & -0.477606 & 0.770154 & 1.195545 \\ \mathrm{C} & 0.400588 & 2.712317 & 0.317260 \\ \mathrm{C} & 1.049408 & 1.678814 & -0.383868 \\ \mathrm{C} & 1.772403 & 1.969987 & -1.538036 \\ \mathrm{C} & 1.887167 & 3.300735 & -1.959017 \\ \mathrm{C} & 1.267412 & 4.321815 & -1.231271 \\ \mathrm{~N} & -1.651905 & 0.404801 & 0.388939 \\ \mathrm{~N} & -3.575770 & -1.104728 & -0.840756 \\ \mathrm{O} & -3.161080 & 1.166434 & 1.908748 \\ \mathrm{H} & -1.129655 & 2.684035 & 1.739100 \\ \mathrm{H} & -1.388497 & 0.531353 & -1.707864 \\ \mathrm{H} & 0.005911 & 4.835266 & 0.454547 \\ \mathrm{H} & -4.216793 & 0.915690 & -0.819594 \\ \mathrm{H} & -5.962178 & 0.495571 & 0.790865 \\ \mathrm{H} & -5.058363 & -0.832676 & 1.531869 \\ \mathrm{H} & -6.409834 & -0.823867 & -1.226330 \\ \mathrm{H} & -6.601510 & -2.040961 & 0.041965 \\ \mathrm{H} & -4.772877 & -2.383446 & -2.032107 \\ \mathrm{H} & -4.414323 & -2.990266 & -0.408660 \\ \mathrm{H} & 0.662022 & -0.717100 & -1.662036 \\ \mathrm{H} & 0.112671 & -1.697521 & -0.305162 \\ \mathrm{H} & -0.549477 & 0.264597 & 2.161421 \\ \mathrm{H} & 2.227703 & 1.178907 & -2.125993 \\ \mathrm{H} & 2.447715 & 3.535982 & -2.858273 \\ \mathrm{H} & 1.358038 & 5.351296 & -1.566411\end{array}$

Figure 11D Cation C4 TS

$\begin{array}{lrrr}11 & & & \\ \mathrm{~N} & 0.892592 & -4.090827 & 0.529078 \\ \mathrm{C} & 1.777601 & -3.249487 & -1.664431 \\ \mathrm{C} & 2.018955 & -1.670858 & 3.109006 \\ \mathrm{C} & 1.638789 & -2.416412 & 1.862048 \\ \mathrm{C} & 0.977610 & -3.642429 & 1.812839 \\ \mathrm{C} & 1.505138 & -3.178773 & -0.305179 \\ \mathrm{C} & 1.961217 & -2.102077 & 0.513988 \\ \mathrm{C} & 2.599192 & -0.983953 & -0.116549 \\ \mathrm{C} & 2.961887 & -1.132875 & -1.495952 \\ \mathrm{C} & 2.532515 & -2.212026 & -2.250864 \\ \mathrm{H} & 0.475488 & -4.966373 & 0.243408 \\ \mathrm{H} & 1.445933 & -4.093877 & -2.261628 \\ \mathrm{H} & 3.098008 & -1.743778 & 3.294257 \\ \mathrm{H} & 1.778683 & -0.603335 & 3.048373 \\ \mathrm{H} & 0.578811 & -4.230334 & 2.628357 \\ \mathrm{H} & 3.183952 & -0.310370 & 0.500948 \\ \mathrm{H} & 3.548987 & -0.349503 & -1.964127 \\ \mathrm{H} & 2.780881 & -2.270329 & -3.305071 \\ \mathrm{H} & 1.503908 & -2.082405 & 3.981687 \\ \mathrm{~N} & 0.877277 & 1.634879 & 1.617970 \\ \mathrm{C} & -2.382668 & -0.022189 & -1.613931 \\ \mathrm{O} & -2.369497 & -0.762710 & -2.596614 \\ \mathrm{C} & -1.138988 & 0.759152 & -1.172129 \\ \mathrm{C} & 2.252593 & 3.653000 & 1.067768 \\ \mathrm{C} & -3.466295 & 1.038283 & 0.374059 \\ \mathrm{C} & -4.680223 & 0.525629 & 1.160966 \\ \mathrm{C} & -5.652649 & 0.091806 & 0.049634\end{array}$




$\begin{array}{rrrr}\mathrm{C} & -4.743264 & -0.512439 & -1.033525 \\ \mathrm{C} & -2.136050 & 0.951946 & 1.120530 \\ \mathrm{C} & 0.194203 & 0.087720 & -1.573359 \\ \mathrm{C} & 1.106517 & 0.527238 & -0.451602 \\ \mathrm{C} & 0.278622 & 0.566843 & 0.843150 \\ \mathrm{C} & 1.664338 & 2.408851 & 0.802674 \\ \mathrm{C} & 1.839407 & 1.771535 & -0.461240 \\ \mathrm{C} & 2.601426 & 2.395657 & -1.466521 \\ \mathrm{C} & 3.179385 & 3.627205 & -1.203173 \\ \mathrm{C} & 3.004446 & 4.240982 & 0.057713 \\ \mathrm{~N} & -1.056540 & 0.847374 & 0.299980 \\ \mathrm{~N} & -3.462614 & 0.187713 & -0.832449 \\ \mathrm{O} & -2.036173 & 0.985581 & 2.344621 \\ \mathrm{H} & 0.386834 & 1.992808 & 2.427448 \\ \mathrm{H} & -1.192480 & 1.768660 & -1.603507 \\ \mathrm{H} & 2.116861 & 4.142278 & 2.026787 \\ \mathrm{H} & -3.617183 & 2.091681 & 0.089283 \\ \mathrm{H} & -5.090898 & 1.291741 & 1.821788 \\ \mathrm{H} & -4.381381 & -0.328306 & 1.777853 \\ \mathrm{H} & -6.181823 & 0.964177 & -0.349233 \\ \mathrm{H} & -6.401637 & -0.622250 & 0.400857 \\ \mathrm{H} & -5.109518 & -0.348997 & -2.050759 \\ \mathrm{H} & -4.594019 & -1.590177 & -0.898215 \\ \mathrm{H} & 0.536205 & 0.414023 & -2.555652 \\ \mathrm{H} & 0.061352 & -0.997003 & -1.598591 \\ \mathrm{H} & 0.239111 & -0.356026 & 1.423676 \\ \mathrm{H} & 2.728410 & 1.922409 & -2.435738 \\ \mathrm{H} & 3.767317 & 4.127582 & -1.965340 \\ \mathrm{H} & 3.466323 & 5.206774 & 0.241523\end{array}$

Figure 11D Cation C4 Product

$\begin{array}{lrrr}11 & & & \\ \mathrm{~N} & 2.499813 & -3.824961 & 0.324475 \\ \mathrm{C} & 3.240414 & -2.474241 & -1.628323 \\ \mathrm{C} & 1.887050 & -1.440664 & 3.087033 \\ \mathrm{C} & 2.117435 & -2.110956 & 1.761675 \\ \mathrm{C} & 2.179529 & -3.516193 & 1.589501 \\ \mathrm{C} & 2.690420 & -2.646430 & -0.383976 \\ \mathrm{C} & 2.400054 & -1.561244 & 0.508659 \\ \mathrm{C} & 2.491217 & -0.157926 & -0.000060 \\ \mathrm{C} & 3.235531 & -0.069695 & -1.295293 \\ \mathrm{C} & 3.540679 & -1.145604 & -2.069769 \\ \mathrm{H} & 2.642710 & -4.761672 & -0.032626 \\ \mathrm{H} & 3.494956 & -3.325280 & -2.253717 \\ \mathrm{H} & 0.883857 & -1.646843 & 3.477791 \\ \mathrm{H} & 2.604844 & -1.809441 & 3.827986 \\ \mathrm{H} & 2.026842 & -4.290485 & 2.329839 \\ \mathrm{H} & 2.978562 & 0.478424 & 0.749469 \\ \mathrm{H} & 3.472110 & 0.927466 & -1.649524 \\ \mathrm{H} & 4.029522 & -1.013981 & -3.028695 \\ \mathrm{H} & 2.003283 & -0.356717 & 3.016504 \\ \mathrm{~N} & 0.418483 & 1.663886 & 1.763658 \\ \mathrm{C} & -2.251115 & -0.616107 & -1.626793 \\ \mathrm{O} & -2.046003 & -1.355735 & -2.589684 \\ \mathrm{C} & -1.197493 & 0.376585 & -1.121254\end{array}$

$\begin{array}{rrrr}\mathrm{C} & 0.930396 & 4.033836 & 1.089736 \\ \mathrm{C} & -3.629823 & 0.262390 & 0.261565 \\ \mathrm{C} & -4.788755 & -0.440914 & 0.980792 \\ \mathrm{C} & -5.597000 & -1.049603 & -0.179118 \\ \mathrm{C} & -4.526932 & -1.502278 & -1.187023 \\ \mathrm{C} & -2.355250 & 0.412965 & 1.091204 \\ \mathrm{C} & 0.248118 & -0.042681 & -1.419201 \\ \mathrm{C} & 1.057218 & 0.571821 & -0.260453 \\ \mathrm{C} & 0.109213 & 0.485756 & 0.974583 \\ \mathrm{C} & 0.850327 & 2.657944 & 0.877039 \\ \mathrm{C} & 1.246130 & 2.082563 & -0.345845 \\ \mathrm{C} & 1.703295 & 2.894887 & -1.380464 \\ \mathrm{C} & 1.783943 & 4.278602 & -1.180719 \\ \mathrm{C} & 1.406325 & 4.833864 & 0.046531 \\ \mathrm{~N} & -1.224347 & 0.485088 & 0.346354 \\ \mathrm{~N} & -3.402703 & -0.588546 & -0.923013 \\ \mathrm{O} & -2.352352 & 0.477456 & 2.320872 \\ \mathrm{H} & -0.273033 & 1.900120 & 2.467776 \\ \mathrm{H} & -1.408249 & 1.357324 & -1.571951 \\ \mathrm{H} & 0.619382 & 4.471833 & 2.033510 \\ \mathrm{H} & -3.938922 & 1.270974 & -0.056546 \\ \mathrm{H} & -5.367264 & 0.250911 & 1.596478 \\ \mathrm{H} & -4.390612 & -1.223584 & 1.635052 \\ \mathrm{H} & -6.239261 & -0.286367 & -0.632418 \\ \mathrm{H} & -6.235637 & -1.877045 & 0.140149 \\ \mathrm{H} & -4.847910 & -1.419442 & -2.229189 \\ \mathrm{H} & -4.205314 & -2.536578 & -1.015837 \\ \mathrm{H} & 0.575917 & 0.311442 & -2.397865 \\ \mathrm{H} & 0.319473 & -1.134482 & -1.417442 \\ \mathrm{H} & 0.200347 & -0.412917 & 1.579446 \\ \mathrm{H} & 1.973907 & 2.475251 & -2.345928 \\ \mathrm{H} & 2.132890 & 4.920296 & -1.983649 \\ \mathrm{H} & 1.470196 & 5.908690 & 0.191401\end{array}$

Figure 11D Cation C5 TS

$\begin{array}{lrrr}11 & & & \\ \text { N } & -5.209632 & 0.262283 & -0.586504 \\ \text { C } & -3.203395 & 0.840413 & -1.976389 \\ \text { C } & -4.627184 & -3.106813 & 0.887129 \\ \text { C } & -4.614109 & -1.782017 & 0.187942 \\ \text { C } & -5.597134 & -0.833208 & 0.186381 \\ \text { C } & -3.976616 & 0.047186 & -1.101810 \\ \text { C } & -3.546267 & -1.243534 & -0.632385 \\ \text { C } & -2.301151 & -1.707898 & -1.016250 \\ \mathrm{C} & -1.454746 & -0.860671 & -1.786322 \\ \mathrm{C} & -1.968702 & 0.359614 & -2.342749 \\ \mathrm{H} & -5.777610 & 1.083321 & -0.746843 \\ \mathrm{H} & -3.585522 & 1.780169 & -2.362403 \\ \mathrm{H} & -3.799807 & -3.193684 & 1.602521 \\ \mathrm{H} & -5.561637 & -3.249195 & 1.437454 \\ \mathrm{H} & -6.561623 & -0.834086 & 0.674360 \\ \mathrm{H} & -1.941769 & -2.682704 & -0.699706 \\ \mathrm{H} & -0.577471 & -1.302144 & -2.248819 \\ \mathrm{H} & -1.352023 & 0.925162 & -3.032984 \\ \mathrm{H} & -4.526682 & -3.936026 & 0.176185\end{array}$




$\begin{array}{lrrr}\mathrm{N} & 0.212167 & 2.155357 & -0.599177 \\ \mathrm{C} & 3.064318 & -1.597984 & 0.666610 \\ \mathrm{O} & 2.941516 & -2.816807 & 0.780964 \\ \mathrm{C} & 1.864309 & -0.652221 & 0.798628 \\ \mathrm{C} & -1.371289 & 3.180726 & 1.038707 \\ \mathrm{C} & 4.373960 & 0.491170 & 0.248974 \\ \mathrm{C} & 5.701164 & 0.624974 & -0.510089 \\ \mathrm{C} & 6.525615 & -0.557286 & 0.029508 \\ \mathrm{C} & 5.497739 & -1.688511 & 0.199805 \\ \mathrm{C} & 3.162285 & 1.087500 & -0.463797 \\ \mathrm{C} & 0.515217 & -1.300054 & 0.400845 \\ \mathrm{C} & -0.245062 & -0.095669 & -0.107465 \\ \mathrm{C} & 0.745076 & 0.829190 & -0.832893 \\ \mathrm{C} & -0.741248 & 2.118853 & 0.373120 \\ \mathrm{C} & -1.049099 & 0.759509 & 0.704185 \\ \mathrm{C} & -1.973371 & 0.475660 & 1.734178 \\ \mathrm{C} & -2.581684 & 1.527568 & 2.395690 \\ \mathrm{C} & -2.282206 & 2.863342 & 2.037875 \\ \mathrm{~N} & 1.982266 & 0.503264 & -0.116804 \\ \mathrm{~N} & 4.232324 & -0.967634 & 0.425187 \\ \mathrm{O} & 3.227386 & 2.015536 & -1.264898 \\ \mathrm{H} & 0.721965 & 2.979367 & -0.886630 \\ \mathrm{H} & 1.813658 & -0.299732 & 1.838190 \\ \mathrm{H} & -1.139534 & 4.211872 & 0.793522 \\ \mathrm{H} & 4.449869 & 0.981310 & 1.232721 \\ \mathrm{H} & 6.171736 & 1.595531 & -0.340323 \\ \mathrm{H} & 5.519506 & 0.519766 & -1.584766 \\ \mathrm{H} & 6.963572 & -0.299222 & 1.000007 \\ \mathrm{H} & 7.340634 & -0.842364 & -0.640232 \\ \mathrm{H} & 5.713278 & -2.349326 & 1.043872 \\ \mathrm{H} & 5.407519 & -2.308657 & -0.699634 \\ \mathrm{H} & 0.031034 & -1.787954 & 1.247248 \\ \mathrm{H} & 0.690544 & -2.048660 & -0.375847 \\ \mathrm{H} & 0.887564 & 0.661779 & -1.902258 \\ \mathrm{H} & -2.193115 & -0.551810 & 2.003906 \\ \mathrm{H} & -3.290257 & 1.334059 & 3.193926 \\ \mathrm{H} & -2.773446 & 3.671477 & 2.572534\end{array}$

Figure 11D Cation C5 Product 11

$\begin{array}{lrrr}\text { N } & -5.647849 & -0.163982 & -1.064375 \\ \mathrm{C} & -3.500934 & 0.740552 & -1.956776 \\ \mathrm{C} & -4.894590 & -3.074322 & 1.141437 \\ \mathrm{C} & -4.942774 & -1.893042 & 0.225743 \\ \mathrm{C} & -6.037991 & -1.219164 & -0.210976 \\ \mathrm{C} & -4.324256 & -0.131559 & -1.192630 \\ \mathrm{C} & -3.798900 & -1.219538 & -0.384328 \\ \mathrm{C} & -2.456582 & -1.414697 & -0.332673 \\ \mathrm{C} & -1.518574 & -0.539144 & -1.089870 \\ \mathrm{C} & -2.160947 & 0.538712 & -1.905149 \\ \mathrm{H} & -6.294610 & 0.473339 & -1.515326 \\ \mathrm{H} & -3.939724 & 1.530300 & -2.557389 \\ \mathrm{H} & -4.317288 & -2.850763 & 2.046276 \\ \mathrm{H} & -5.901210 & -3.371654 & 1.446142 \\ \mathrm{H} & -7.086674 & -1.376641 & -0.005002\end{array}$

$\begin{array}{rrrr}\mathrm{H} & -2.035003 & -2.228748 & 0.249905 \\ \mathrm{H} & -1.003861 & -1.209824 & -1.804330 \\ \mathrm{H} & -1.499965 & 1.188129 & -2.468717 \\ \mathrm{H} & -4.418714 & -3.933438 & 0.654255 \\ \mathrm{~N} & 0.411151 & 2.171135 & -0.902376 \\ \mathrm{C} & 2.930070 & -1.608562 & 0.817419 \\ \mathrm{O} & 2.713867 & -2.766109 & 1.176857 \\ \mathrm{C} & 1.821497 & -0.550925 & 0.777156 \\ \mathrm{C} & -0.570374 & 3.548170 & 0.963059 \\ \mathrm{C} & 4.396394 & 0.225560 & -0.041922 \\ \mathrm{C} & 5.677309 & 0.072355 & -0.874022 \\ \mathrm{C} & 6.431205 & -1.050119 & -0.139169 \\ \mathrm{C} & 5.322973 & -2.015876 & 0.315540 \\ \mathrm{C} & 3.204720 & 0.803108 & -0.802065 \\ \mathrm{C} & 0.421922 & -1.128088 & 0.538595 \\ \mathrm{C} & -0.343655 & 0.013358 & -0.170655 \\ \mathrm{C} & 0.746260 & 0.759845 & -1.015439 \\ \mathrm{C} & -0.300573 & 2.356339 & 0.289966 \\ \mathrm{C} & -0.805694 & 1.130001 & 0.756669 \\ \mathrm{C} & -1.594022 & 1.084204 & 1.900962 \\ \mathrm{C} & -1.873019 & 2.274665 & 2.585310 \\ \mathrm{C} & -1.361803 & 3.488679 & 2.114921 \\ \mathrm{~N} & 1.999859 & 0.370399 & -0.353815 \\ \mathrm{~N} & 4.138339 & -1.147837 & 0.431055 \\ \mathrm{O} & 3.324239 & 1.606783 & -1.726776 \\ \mathrm{H} & 1.163233 & 2.810349 & -1.137089 \\ \mathrm{H} & 1.846015 & 0.006585 & 1.725035 \\ \mathrm{H} & -0.173798 & 4.493987 & 0.605835 \\ \mathrm{H} & 4.585667 & 0.887280 & 0.818737 \\ \mathrm{H} & 6.239544 & 1.006379 & -0.934107 \\ \mathrm{H} & 5.416362 & -0.232292 & -1.893098 \\ \mathrm{H} & 6.955170 & -0.643242 & 0.732794 \\ \mathrm{H} & 7.171380 & -1.545374 & -0.772529 \\ \mathrm{H} & 5.535184 & -2.500659 & 1.272690 \\ \mathrm{H} & 5.126546 & -2.802344 & -0.422599 \\ \mathrm{H} & -0.048612 & -1.432863 & 1.475099 \\ \mathrm{H} & 0.499505 & -2.011127 & -0.104720 \\ \mathrm{H} & 0.800100 & 0.467323 & -2.067675 \\ \mathrm{H} & -1.982723 & 0.140223 & 2.275221 \\ \mathrm{H} & -2.480487 & 2.252143 & 3.484775 \\ \mathrm{H} & -1.577357 & 4.406476 & 2.655127\end{array}$

Figure 11D Cation C6 TS 11

$\begin{array}{lrrr}\text { N } & 1.379894 & -3.637013 & -1.373907 \\ \mathrm{C} & 1.821328 & -1.235931 & -1.944873 \\ \mathrm{C} & 3.139838 & -4.283304 & 1.837828 \\ \mathrm{C} & 2.536687 & -3.741532 & 0.576205 \\ \mathrm{C} & 1.760788 & -4.442501 & -0.338054 \\ \mathrm{C} & 1.898664 & -2.372763 & -1.170144 \\ \mathrm{C} & 2.642216 & -2.421301 & 0.061929 \\ \mathrm{C} & 3.320239 & -1.254113 & 0.498758 \\ \mathrm{C} & 3.220312 & -0.104315 & -0.246637 \\ \mathrm{C} & 2.415827 & -0.043266 & -1.442852 \\ \mathrm{H} & 0.812567 & -3.924355 & -2.159092\end{array}$




$\begin{array}{rrrr}\mathrm{H} & 1.316476 & -1.239775 & -2.905585 \\ \mathrm{H} & 2.770154 & -3.748907 & 2.721254 \\ \mathrm{H} & 2.898096 & -5.342637 & 1.963479 \\ \mathrm{H} & 1.457412 & -5.480661 & -0.313096 \\ \mathrm{H} & 3.913588 & -1.274587 & 1.407704 \\ \mathrm{H} & 3.732973 & 0.796623 & 0.074403 \\ \mathrm{H} & 2.628675 & 0.743028 & -2.160472 \\ \mathrm{H} & 4.232254 & -4.187711 & 1.833871 \\ \mathrm{~N} & 0.856189 & 1.366652 & 1.603421 \\ \mathrm{C} & -2.840085 & 0.402586 & -1.391288 \\ \mathrm{O} & -3.000248 & 0.208750 & -2.595803 \\ \mathrm{C} & -1.621545 & 1.162492 & -0.851927 \\ \mathrm{C} & 1.912636 & 3.628959 & 1.704735 \\ \mathrm{C} & -3.479274 & 0.169408 & 1.016139 \\ \mathrm{C} & -4.358255 & -0.921963 & 1.642260 \\ \mathrm{C} & -5.533341 & -1.025757 & 0.653863 \\ \mathrm{C} & -4.885486 & -0.829530 & -0.727306 \\ \mathrm{C} & -2.000253 & 0.091164 & 1.387074 \\ \mathrm{C} & -0.370407 & 1.043793 & -1.755959 \\ \mathrm{C} & 0.741080 & 1.168523 & -0.732113 \\ \mathrm{C} & 0.284477 & 0.518083 & 0.577509 \\ \mathrm{C} & 1.381873 & 2.497805 & 1.063005 \\ \mathrm{C} & 1.317859 & 2.428317 & -0.364505 \\ \mathrm{C} & 1.768525 & 3.510759 & -1.147381 \\ \mathrm{C} & 2.283517 & 4.625237 & -0.510648 \\ \mathrm{C} & 2.355020 & 4.671782 & 0.903838 \\ \mathrm{~N} & -1.170401 & 0.601116 & 0.437855 \\ \mathrm{~N} & -3.691223 & -0.017702 & -0.432439 \\ \mathrm{O} & -1.587841 & -0.370302 & 2.447562 \\ \mathrm{H} & 0.725152 & 1.182685 & 2.587708 \\ \mathrm{H} & -1.895665 & 2.219484 & -0.727410 \\ \mathrm{H} & 1.964765 & 3.685832 & 2.786908 \\ \mathrm{H} & -3.839473 & 1.164888 & 1.321077 \\ \mathrm{H} & -4.666397 & -0.664652 & 2.657624 \\ \mathrm{H} & -3.797401 & -1.861530 & 1.686459 \\ \mathrm{H} & -6.257926 & -0.226641 & 0.845105 \\ \mathrm{H} & -6.061040 & -1.979895 & 0.726558 \\ \mathrm{H} & -5.531074 & -0.310172 & -1.440897 \\ \mathrm{H} & -4.576453 & -1.778114 & -1.181795 \\ \mathrm{H} & -0.336606 & 1.825350 & -2.515943 \\ \mathrm{H} & -0.388192 & 0.075090 & -2.254470 \\ \mathrm{H} & 0.586660 & -0.521057 & 0.734806 \\ \mathrm{H} & 1.718772 & 3.463195 & -2.231372 \\ \mathrm{H} & 2.637675 & 5.470995 & -1.090336 \\ \mathrm{H} & 2.765062 & 5.558780 & 1.378192\end{array}$

Figure 11D Cation C6 Product

$\begin{array}{lrrr}11 & & & \\ \text { N } & 3.408693 & -3.005977 & -0.939452 \\ \text { C } & 2.053440 & -0.970920 & -1.398005 \\ \text { C } & 6.033981 & -1.979417 & 1.484378 \\ \text { C } & 4.882407 & -2.082431 & 0.529516 \\ \text { C } & 4.474648 & -3.245005 & -0.154230 \\ \text { C } & 3.052001 & -1.664323 & -0.808390 \\ \text { C } & 3.996354 & -1.078262 & 0.124837\end{array}$

\begin{tabular}{rrrr}
$\mathrm{C}$ & 3.878330 & 0.310349 & 0.440239 \\
$\mathrm{C}$ & 2.882710 & 1.037455 & -0.125871 \\
$\mathrm{C}$ & 1.887275 & 0.481015 & -1.098684 \\
$\mathrm{H}$ & 2.941379 & -3.690966 & -1.519809 \\
$\mathrm{H}$ & 1.389910 & -1.447578 & -2.110866 \\
$\mathrm{H}$ & 5.697763 & -1.661297 & 2.477523 \\
$\mathrm{H}$ & 6.538637 & -2.942927 & 1.593274 \\
$\mathrm{H}$ & 4.911716 & -4.234404 & -0.106518 \\
$\mathrm{H}$ & 4.578348 & 0.769932 & 1.130242 \\
$\mathrm{H}$ & 2.785547 & 2.091581 & 0.107871 \\
$\mathrm{H}$ & 2.065656 & 1.000661 & -2.060092 \\
$\mathrm{H}$ & 6.773451 & -1.249310 & 1.136897 \\
$\mathrm{~N}$ & 0.177992 & 1.414163 & 1.598650 \\
$\mathrm{C}$ & -2.947730 & -0.723520 & -1.426272 \\
$\mathrm{O}$ & -2.970913 & -1.104816 & -2.596910 \\
$\mathrm{C}$ & -1.961199 & 0.341614 & -0.934745 \\
$\mathrm{C}$ & -0.004084 & 3.919403 & 1.407065 \\
$\mathrm{C}$ & -3.708189 & -0.778640 & 0.953976 \\
$\mathrm{C}$ & -4.381505 & -1.948959 & 1.685247 \\
$\mathrm{C}$ & -5.431333 & -2.432229 & 0.668820 \\
$\mathrm{C}$ & -4.738131 & -2.273312 & -0.695260 \\
$\mathrm{C}$ & -2.278180 & -0.480786 & 1.401304 \\
$\mathrm{C}$ & -0.662961 & 0.405913 & -1.748730 \\
$\mathrm{C}$ & 0.391645 & 0.900705 & -0.731137 \\
$\mathrm{C}$ & -0.069004 & 0.335500 & 0.653666 \\
$\mathrm{C}$ & 0.125706 & 2.624596 & 0.902021 \\
$\mathrm{C}$ & 0.291895 & 2.399057 & -0.476163 \\
$\mathrm{C}$ & 0.347437 & 3.467312 & -1.364956 \\
$\mathrm{C}$ & 0.220086 & 4.771137 & -0.869504 \\
$\mathrm{C}$ & 0.044457 & 4.984904 & 0.502525 \\
$\mathrm{H}$ & -1.491079 & 0.036762 & 0.425616 \\
$\mathrm{~N}$ & -3.769180 & -1.189200 & -0.461872 \\
$\mathrm{O}$ & -1.880476 & -0.675783 & 2.549978 \\
$\mathrm{H}$ & -0.295920 & 1.327302 & 2.491272 \\
$\mathrm{H}$ & -2.470952 & 1.315998 & -0.947957 \\
$\mathrm{H}$ & -0.139409 & 4.094207 & 2.470280 \\
$\mathrm{H}$ & -4.294332 & 0.142794 & 1.100847 \\
$\mathrm{H}$ & -4.811618 & -1.640823 & 2.640351 \\
$\mathrm{H}$ & -3.639679 & -2.729683 & 1.884632 \\
$\mathrm{H}$ & -6.320705 & -1.793942 & 0.714074 \\
$\mathrm{H}$ & -5.749956 & -3.462009 & 0.848926 \\
$\mathrm{H}$ & -5.424521 & -2.003515 & -1.502847 \\
$\mathrm{H}$ & -4.203194 & -3.180952 & -0.999167 \\
$\mathrm{H}$ & -0.750634 & 1.075766 & -2.606406 \\
\hline
\end{tabular}

Figure 11D Cation C7 TS

$\begin{array}{lrrr}11 & & & \\ \text { N } & 2.280527 & -2.430600 & -0.931170 \\ \text { C } & 1.526461 & -0.246925 & -1.968903 \\ \text { C } & 5.685665 & -1.855176 & 0.455900\end{array}$




\begin{tabular}{|c|c|c|c|}
\hline & & & \\
\hline & & & \\
\hline & 075 & & \\
\hline & 55 & & \\
\hline & 6175 & 68985 & ברחמתח 19 \\
\hline & 5302 & 359 & -10 \\
\hline & 2932 & 1654 & -2 \\
\hline & 2917 & 3486 & \\
\hline & 0.662988 & 668 & -2.4 \\
\hline & 6952 & 8077 & \\
\hline & 5.896967 & 41173 & 0.8 \\
\hline & 7545 & 8199 & \\
\hline & 5.124753 & 562 & \\
\hline & 3.727998 & & \\
\hline & & & \\
\hline & & & \\
\hline & & & \\
\hline & & & \\
\hline & & & \\
\hline & & & \\
\hline & & & \\
\hline C & & & \\
\hline C & & & \\
\hline & & & \\
\hline & -5.5 & & \\
\hline & -2.6 & & \\
\hline & -0.7 & & \\
\hline & & & \\
\hline & -0.2 & & \\
\hline & & & \\
\hline & 1.1 & & \\
\hline & & & \\
\hline & & & \\
\hline & & & \\
\hline & -1.6 & & \\
\hline & -4.2 & & -0 . \\
\hline & -2.3 & & \\
\hline & & & \\
\hline & -2.0 & & \\
\hline & & & \\
\hline$H$ & -4.1 & & \\
\hline $\mathrm{H}$ & & & \\
\hline $\mathrm{H}$ & -4.771336 & & 0.232 \\
\hline $\mathrm{H}$ & -6.830416 & & 0.8 \\
\hline $\mathrm{H}$ & -7.003803 & -1 & -0.3 \\
\hline $\mathrm{H}$ & -6.111736 & & -1.1 \\
\hline 11 & -5.484594 & 2820 & -1.91722 \\
\hline $\mathrm{H}$ & -0.582640 & 2.383334 & -1.35390 \\
\hline & -0.985565 & $0 . \varepsilon$ & -2.16301 \\
\hline & -0.231335 & 4047 & -0.233 \\
\hline & & & -0.297 \\
\hline & & & \\
\hline & 2.90 & 76 & 2771 \\
\hline
\end{tabular}

Figure 11D Cation C7 Product

\begin{tabular}{|c|c|c|c|}
\hline & & & \\
\hline & 502525 & & \\
\hline & 6.406364 & 0.2 & \\
\hline & & 590 & \\
\hline & 0745 & -0.7 & \\
\hline & 2.7 & & \\
\hline & & & \\
\hline & & & \\
\hline & & & \\
\hline & 2.064278 & & \\
\hline & & -1.0 & \\
\hline & & & \\
\hline & & & \\
\hline & & & \\
\hline & & & \\
\hline & & & \\
\hline & & & \\
\hline & & & \\
\hline & & & \\
\hline & & & \\
\hline & & & \\
\hline & & & \\
\hline & & & \\
\hline & & & \\
\hline & & & \\
\hline & & & \\
\hline & & & \\
\hline & & & \\
\hline & & -0 & \\
\hline & -0. & & \\
\hline & & & \\
\hline & -0 & & \\
\hline & & & \\
\hline & & & \\
\hline & & & \\
\hline C & & & \\
\hline 乙 & & -3 & \\
\hline 1 & & -0 & \\
\hline $\mathrm{N}$ & & & \\
\hline O & & & \\
\hline $\mathrm{H}$ & & -2 & \\
\hline & & -0.2 & \\
\hline & & -4 & \\
\hline & -4 & & \\
\hline & & & \\
\hline & & & \\
\hline & & & \\
\hline & & & \\
\hline & & & \\
\hline & & & \\
\hline & & & \\
\hline & & & \\
\hline & & & \\
\hline & & 0.136146 & \\
\hline
\end{tabular}




$$
\begin{array}{lll}
\mathrm{H} & 2.169476 & -2.055530 \\
\mathrm{H} & 1.866728 & -4.143663
\end{array}
$$

4.064145

2.766266

Figure 12B Radical C2 TS

\begin{tabular}{|c|c|c|c|}
\hline & 907 & .004 & \\
\hline 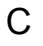 & 886 & 3709 & \\
\hline & 1.469529 & 0.110404 & 004 \\
\hline & .550238 & 0.084548 & -1.35060 \\
\hline & .239943 & 1.226885 & -0.82970 \\
\hline & 4.163016 & -0.595537 & 0.14435 \\
\hline & 3.086290 & -1.050405 & -0.67759 \\
\hline & 2.757418 & 3324 & 279 \\
\hline & 3.500216 & 1740 & 0. \\
\hline & 57 & 625 & 0. \\
\hline & & & \\
\hline & & & \\
\hline & & 991 & \\
\hline & 50 & 93 & \\
\hline & 19 & 39 & \\
\hline & 1.91 & -2.7 & -1 \\
\hline & 50 & -4 & \\
\hline & 0 & & \\
\hline & 1.8 & $-0 .($ & \\
\hline & 3 & & \\
\hline & 61 & -0.2 & \\
\hline & -3.6 & & \\
\hline & -1.67 & 355 & \\
\hline & 0.79 & 603 & \\
\hline & -2.96 & -2 & \\
\hline 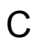 & -3.668698 & -2.3 & \\
\hline C & -5.092964 & -1.8 & \\
\hline C & -4.887071 & 0721 & -0 . \\
\hline C & -1.446904 & -2.022192 & -0 . \\
\hline C & -0.847828 & 4949 & 319 \\
\hline 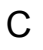 & -0.001896 & 1.2 & 53 \\
\hline 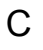 & 1.367320 & & \\
\hline C & 0.807731 & 4924 & -0 . \\
\hline C & -0.398600 & 4215 & 20 \\
\hline C & -1.612984 & 0760 & 0. \\
\hline C & -1.617812 & 0544 & -0 . \\
\hline$r_{1}+2$ & -0.429857 & 4052 & -1 \\
\hline N & & & \\
\hline 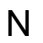 & -3.5 & & \\
\hline 0 & & & \\
\hline $\mathrm{H}$ & & & \\
\hline & & & \\
\hline $\mathrm{H}$ & -3.1 & & \\
\hline $\mathrm{H}$ & -3.6 & & \\
\hline $\mathrm{H}$ & -3.1 & -1 & -2 \\
\hline $\mathrm{H}$ & -5.67 & 449 & $-0 . \varepsilon$ \\
\hline $\mathrm{H}$ & -5.628260 & & \\
\hline $\mathrm{H}$ & -5.67 & & \\
\hline $\mathrm{H}$ & -4.81 & 0.3 & -0.7 \\
\hline & 192695 & (2012638 & \\
\hline
\end{tabular}
02

$\begin{array}{rrrr}\mathrm{H} & -1.570835 & 1.065841 & 2.996706 \\ \mathrm{H} & 2.026677 & 0.485201 & 1.935393 \\ \mathrm{H} & -2.533542 & 2.614188 & 0.747398 \\ \mathrm{H} & -2.555362 & 4.390831 & -0.990725 \\ \mathrm{H} & -0.463113 & 5.096279 & -2.110883 \\ \mathrm{H} & 0.079974 & -1.142087 & 0.672604\end{array}$

Figure 12B Radical C2 Product

$\begin{array}{lrrr}\text { O 2 } & & & \\ \mathrm{N} & 3.844035 & 1.208031 & 0.392991 \\ \mathrm{C} & 5.979825 & 0.026852 & 0.966909 \\ \mathrm{C} & 2.066650 & -1.142762 & -1.962229 \\ \mathrm{C} & 3.000126 & -0.435724 & -1.039688 \\ \mathrm{C} & 2.768026 & 0.991404 & -0.574977 \\ \mathrm{C} & 4.750605 & 0.158412 & 0.336072 \\ \mathrm{C} & 4.213103 & -0.884897 & -0.491898 \\ \mathrm{C} & 4.940639 & -2.091698 & -0.628897 \\ \mathrm{C} & 6.169757 & -2.220855 & 0.011042 \\ \mathrm{C} & 6.688163 & -1.174479 & 0.794231 \\ \mathrm{H} & 4.172928 & 2.153139 & 0.544434 \\ \mathrm{H} & 6.381942 & 0.827225 & 1.581875 \\ \mathrm{H} & 1.117781 & -1.413151 & -1.474894 \\ \mathrm{H} & 1.803963 & -0.516444 & -2.826407 \\ \mathrm{H} & 2.842330 & 1.695276 & -1.417127 \\ \mathrm{H} & 4.548778 & -2.899226 & -1.241099 \\ \mathrm{H} & 6.739514 & -3.139437 & -0.098750 \\ \mathrm{H} & 7.652607 & -1.294885 & 1.279722 \\ \mathrm{H} & 2.512054 & -2.067489 & -2.342193 \\ \mathrm{~N} & 1.444723 & 1.226250 & 0.007224 \\ \mathrm{C} & -3.533280 & -0.129704 & 1.363734 \\ \mathrm{O} & -3.985056 & 0.915990 & 1.839997 \\ \mathrm{C} & -2.247140 & -0.766567 & 1.917209 \\ \mathrm{C} & 0.510642 & 3.056465 & -1.474559 \\ \mathrm{C} & -3.702065 & -2.090105 & -0.187034 \\ \mathrm{C} & -4.305053 & -2.080432 & -1.596318 \\ \mathrm{C} & -5.644268 & -1.351322 & -1.382075 \\ \mathrm{C} & -5.332222 & -0.274670 & -0.324546 \\ \mathrm{C} & -2.190230 & -2.251469 & -0.120179 \\ \mathrm{C} & -1.233719 & 0.305783 & 2.411996 \\ \mathrm{C} & -0.362705 & 0.908528 & 1.344848 \\ \mathrm{C} & 0.925800 & 0.515953 & 1.074252 \\ \mathrm{C} & 0.475985 & 2.114281 & -0.437185 \\ \mathrm{C} & -0.676918 & 1.941487 & 0.383658 \\ \mathrm{C} & -1.807780 & 2.746514 & 0.150780 \\ \mathrm{C} & -1.771287 & 3.682244 & -0.876068 \\ \mathrm{C} & -0.623890 & 3.833911 & -1.681141 \\ \mathrm{~N} & -1.611115 & -1.664085 & 0.954180 \\ \mathrm{~N} & -4.142951 & -0.800601 & 0.366916 \\ \mathrm{O} & -1.562198 & -2.898852 & -0.956976 \\ \mathrm{H} & -2.562383 & -1.338462 & 2.804027 \\ \mathrm{H} & 1.387585 & 3.186355 & -2.101654 \\ \mathrm{H} & -4.143306 & -2.914172 & 0.397482 \\ \mathrm{H} & -4.419285 & -3.088082 & -2.001271 \\ \mathrm{H} & -3.651645 & -1.515131 & -2.269430 \\ & -6.393717 & -2.050965 & -0.995428\end{array}$




$\begin{array}{rrrr}H & -6.040334 & -0.915881 & -2.303065 \\ H & -6.151085 & -0.115730 & 0.383671 \\ H & -5.086018 & 0.694771 & -0.772079 \\ H & -0.592224 & -0.171965 & 3.161121 \\ H & -1.823171 & 1.067385 & 2.930569 \\ H & 1.543313 & -0.213996 & 1.581086 \\ H & -2.695993 & 2.624565 & 0.762416 \\ H & -2.639825 & 4.307915 & -1.064014 \\ H & -0.622314 & 4.572646 & -2.478131 \\ H & -0.598896 & -1.721430 & 0.978165\end{array}$

Figure 12B Radical C3 TS

02

$\begin{array}{lrrr}\text { N } & -1.367504 & -2.194245 & -1.417445 \\ \mathrm{C} & -0.989037 & -2.879502 & 0.973255 \\ \mathrm{C} & -4.437493 & -0.173647 & -1.409041 \\ \mathrm{C} & -3.024797 & -0.611376 & -1.096303 \\ \mathrm{C} & -2.266328 & -1.386171 & -2.056654 \\ \mathrm{C} & -1.600025 & -2.150108 & -0.045200 \\ \mathrm{C} & -2.676354 & -1.267717 & 0.194065 \\ \mathrm{C} & -3.199069 & -1.167409 & 1.483573 \\ \mathrm{C} & -2.610847 & -1.907692 & 2.514168 \\ \mathrm{C} & -1.508852 & -2.738778 & 2.262411 \\ \mathrm{H} & -0.493810 & -2.539001 & -1.813588 \\ \mathrm{H} & -0.157926 & -3.545046 & 0.763001 \\ \mathrm{H} & -4.480924 & 0.313878 & -2.387122 \\ \mathrm{H} & -2.268920 & -1.295329 & -3.133111 \\ \mathrm{H} & -4.053648 & -0.531918 & 1.692058 \\ \mathrm{H} & -3.011317 & -1.837459 & 3.521220 \\ \mathrm{H} & -1.062355 & -3.298924 & 3.079357 \\ \mathrm{H} & -5.108143 & -1.040940 & -1.421154 \\ \mathrm{~N} & -2.128709 & 1.056529 & -1.067112 \\ \mathrm{C} & 2.982124 & 1.195665 & -0.255042 \\ \mathrm{O} & 3.150924 & 2.296986 & 0.272868 \\ \mathrm{C} & 2.426933 & 1.084905 & -1.682423 \\ \mathrm{C} & -2.623481 & 2.069911 & 1.195555 \\ \mathrm{C} & 3.209738 & -1.303534 & -0.236116 \\ \mathrm{C} & 3.024921 & -2.207772 & 0.987990 \\ \mathrm{C} & 3.907436 & -1.516631 & 2.044072 \\ \mathrm{C} & 3.737519 & -0.009930 & 1.763954 \\ \mathrm{C} & 2.131030 & -1.394779 & -1.302253 \\ \mathrm{C} & 1.335834 & 2.160024 & -1.956246 \\ \mathrm{C} & 0.001701 & 1.892822 & -1.317166 \\ \mathrm{C} & -1.008121 & 1.139953 & -1.869889 \\ \mathrm{C} & -1.822735 & 1.775768 & 0.083028 \\ \mathrm{C} & -0.504623 & 2.323189 & -0.033321 \\ \mathrm{C} & -0.000810 & 3.146953 & 0.986492 \\ \mathrm{C} & -0.796395 & 3.410324 & 2.098568 \\ \mathrm{C} & -2.097520 & 2.880335 & 2.199075 \\ \mathrm{~N} & 1.906496 & -0.252401 & -1.982111 \\ \mathrm{~N} & 3.294394 & 0.036407 & 0.359950 \\ \mathrm{O} & 1.553682 & -2.460824 & -1.552065 \\ \mathrm{H} & 3.271986 & 1.302780 & -2.353019 \\ \mathrm{H} & -3.639157 & 1.695538 & 1.269980 \\ \mathrm{H} & 4.163484 & -1.550864 & -0.731696\end{array}$

$\begin{array}{rrrr}\mathrm{H} & 3.317304 & -3.240285 & 0.785432 \\ \mathrm{H} & 1.972841 & -2.200978 & 1.291750 \\ \mathrm{H} & 4.954342 & -1.809143 & 1.906416 \\ \mathrm{H} & 3.621919 & -1.776101 & 3.066655 \\ \mathrm{H} & 4.663649 & 0.558148 & 1.895163 \\ \mathrm{H} & 2.970837 & 0.453162 & 2.394533 \\ \mathrm{H} & 1.215897 & 2.220678 & -3.045393 \\ \mathrm{H} & 1.757207 & 3.114781 & -1.630032 \\ \mathrm{H} & -1.011410 & 0.662208 & -2.841853 \\ \mathrm{H} & 1.000340 & 3.560597 & 0.908708 \\ \mathrm{H} & -0.414439 & 4.043563 & 2.895535 \\ \mathrm{H} & -2.706362 & 3.115062 & 3.068594 \\ \mathrm{H} & 1.190629 & -0.312012 & -2.697214 \\ \mathrm{H} & -4.806097 & 0.535473 & -0.662920\end{array}$

Figure 12B Radical C3 Product 02

$\begin{array}{lrrr}\mathrm{N} & 4.312527 & 1.164820 & -1.750030 \\ \mathrm{C} & 4.542382 & 2.792893 & 0.148514 \\ \mathrm{C} & 4.272968 & -1.869107 & -0.314840 \\ \mathrm{C} & 3.342897 & -0.665966 & -0.606490 \\ \mathrm{C} & 3.715606 & -0.069375 & -1.953026 \\ \mathrm{C} & 4.183567 & 1.574510 & -0.430173 \\ \mathrm{C} & 3.604522 & 0.531754 & 0.320371 \\ \mathrm{C} & 3.382529 & 0.712228 & 1.680089 \\ \mathrm{C} & 3.737655 & 1.929878 & 2.279454 \\ \mathrm{C} & 4.311985 & 2.953330 & 1.517530 \\ \mathrm{H} & 4.609874 & 1.772308 & -2.500012 \\ \mathrm{H} & 4.982995 & 3.588816 & -0.445067 \\ \mathrm{H} & 4.087569 & -2.668154 & -1.040586 \\ \mathrm{H} & 3.992059 & -0.661481 & -2.817333 \\ \mathrm{H} & 2.925877 & -0.068848 & 2.279010 \\ \mathrm{H} & 3.561862 & 2.078037 & 3.340732 \\ \mathrm{H} & 4.581626 & 3.892306 & 1.993452 \\ \mathrm{H} & 5.312712 & -1.543372 & -0.413251 \\ \mathrm{~N} & 1.909026 & -1.103912 & -0.600893 \\ \mathrm{C} & -3.580380 & -0.585581 & -0.867267 \\ \mathrm{O} & -3.876331 & -1.783690 & -0.851375 \\ \mathrm{C} & -2.487219 & -0.012732 & -1.790841 \\ \mathrm{C} & 1.621370 & -2.396346 & 1.585685 \\ \mathrm{C} & -3.936443 & 1.785607 & -0.173889 \\ \mathrm{C} & -4.518354 & 2.297934 & 1.148973 \\ \mathrm{C} & -5.732023 & 1.376386 & 1.367507 \\ \mathrm{C} & -5.263573 & 0.002504 & 0.855260 \\ \mathrm{C} & -2.448482 & 2.055463 & -0.380635 \\ \mathrm{C} & -1.441295 & -1.073918 & -2.227519 \\ \mathrm{C} & -0.248495 & -1.251616 & -1.328071 \\ \mathrm{C} & 1.006845 & -0.761254 & -1.593018 \\ \mathrm{C} & 1.214377 & -1.830056 & 0.365258 \\ \mathrm{C} & -0.141860 & -1.947265 & -0.071061 \\ \mathrm{C} & -1.067940 & -2.663125 & 0.710433 \\ \mathrm{C} & -0.650200 & -3.231219 & 1.906279 \\ \mathrm{C} & 0.682336 & -3.091263 & 2.340230 \\ \mathrm{~N} & -1.839889 & 1.149772 & -1.184398 \\ \mathrm{~N} & -4.217062 & 0.340336 & -0.122523\end{array}$




$\begin{array}{rrrr}\mathrm{O} & -1.874691 & 3.016219 & 0.127380 \\ \mathrm{H} & -3.019281 & 0.300436 & -2.704074 \\ \mathrm{H} & 2.635330 & -2.304512 & 1.953866 \\ \mathrm{H} & -4.477184 & 2.238695 & -1.021203 \\ \mathrm{H} & -4.781510 & 3.356652 & 1.098508 \\ \mathrm{H} & -3.777790 & 2.171160 & 1.945698 \\ \mathrm{H} & -6.581887 & 1.727721 & 0.771497 \\ \mathrm{H} & -6.051070 & 1.337284 & 2.412298 \\ \mathrm{H} & -6.059219 & -0.578240 & 0.379285 \\ \mathrm{H} & -4.830885 & -0.613805 & 1.652177 \\ \mathrm{H} & -1.081470 & -0.788848 & -3.222082 \\ \mathrm{H} & -1.993269 & -2.011971 & -2.343116 \\ \mathrm{H} & 1.353218 & -0.199463 & -2.448999 \\ \mathrm{H} & -2.096900 & -2.755239 & 0.376850 \\ \mathrm{H} & -1.357434 & -3.784974 & 2.518139 \\ \mathrm{H} & 0.989424 & -3.533306 & 3.284327 \\ \mathrm{H} & -0.832258 & 1.226309 & -1.275306 \\ \mathrm{H} & 4.146923 & -2.276845 & 0.689539\end{array}$

Figure 12B Radical C4 TS 02

$\begin{array}{lrrr}\mathrm{N} & 4.667825 & -2.445472 & 0.666480 \\ \mathrm{C} & 2.510785 & -2.607687 & -0.629545 \\ \mathrm{C} & 5.838701 & 1.059626 & 0.315739 \\ \mathrm{C} & 5.151211 & -0.272539 & 0.286677 \\ \mathrm{C} & 5.574211 & -1.438945 & 0.902173 \\ \mathrm{C} & 3.640375 & -1.957338 & -0.113353 \\ \mathrm{C} & 3.924487 & -0.593911 & -0.367220 \\ \mathrm{C} & 2.988180 & 0.192686 & -1.126304 \\ \mathrm{C} & 1.916536 & -0.528038 & -1.760699 \\ \mathrm{C} & 1.657648 & -1.861398 & -1.464770 \\ \mathrm{H} & 4.758690 & -3.397934 & 0.988548 \\ \mathrm{H} & 2.310627 & -3.653187 & -0.414748 \\ \mathrm{H} & 5.179257 & 1.835282 & 0.723065 \\ \mathrm{H} & 6.743096 & 1.025635 & 0.931393 \\ \mathrm{H} & 6.459963 & -1.622455 & 1.494680 \\ \mathrm{H} & 3.350839 & 1.094784 & -1.606459 \\ \mathrm{H} & 1.248796 & 0.017521 & -2.419827 \\ \mathrm{H} & 0.776622 & -2.340489 & -1.881092 \\ \mathrm{H} & 6.133009 & 1.385504 & -0.690404 \\ \mathrm{~N} & 2.011978 & 1.289791 & 0.226097 \\ \mathrm{C} & -3.297860 & 0.201890 & 1.322863 \\ \mathrm{O} & -3.607835 & 1.293846 & 1.806634 \\ \mathrm{C} & -2.026045 & -0.561626 & 1.742974 \\ \mathrm{C} & 1.241373 & 3.154316 & -1.283442 \\ \mathrm{C} & -3.753772 & -1.782222 & -0.121037 \\ \mathrm{C} & -4.587890 & -1.830645 & -1.406617 \\ \mathrm{C} & -5.836833 & -1.010097 & -1.036187 \\ \mathrm{C} & -5.297520 & 0.119500 & -0.139765 \\ \mathrm{C} & -2.251249 & -1.965704 & -0.315441 \\ \mathrm{C} & -0.931699 & 0.367254 & 2.337892 \\ \mathrm{C} & 0.030014 & 0.956845 & 1.346365 \\ \mathrm{C} & 1.332070 & 0.540058 & 1.161819 \\ \mathrm{C} & 1.073311 & 2.187611 & -0.288031 \\ \mathrm{C} & -0.165707 & 2.046320 & 0.404955\end{array}$

$\begin{array}{rrrr}\mathrm{C} & -1.226791 & 2.913528 & 0.112320 \\ \mathrm{C} & -1.053609 & 3.874225 & -0.886909 \\ \mathrm{C} & 0.163073 & 3.990345 & -1.580817 \\ \mathrm{~N} & -1.500012 & -1.362346 & 0.635679 \\ \mathrm{~N} & -4.064230 & -0.450390 & 0.425479 \\ \mathrm{O} & -1.787059 & -2.618313 & -1.250351 \\ \mathrm{H} & -2.347662 & -1.232525 & 2.555940 \\ \mathrm{H} & 2.186663 & 3.258655 & -1.809853 \\ \mathrm{H} & -4.096956 & -2.561475 & 0.579417 \\ \mathrm{H} & -4.815942 & -2.854686 & -1.709958 \\ \mathrm{H} & -4.030143 & -1.353436 & -2.219223 \\ \mathrm{H} & -6.541412 & -1.631858 & -0.472312 \\ \mathrm{H} & -6.362727 & -0.621792 & -1.912285 \\ \mathrm{H} & -5.987886 & 0.406794 & 0.658893 \\ \mathrm{H} & -5.052029 & 1.023147 & -0.710022 \\ \mathrm{H} & -0.360437 & -0.220160 & 3.064764 \\ \mathrm{H} & -1.465976 & 1.142846 & 2.895954 \\ \mathrm{H} & 1.847984 & -0.257606 & 1.684792 \\ \mathrm{H} & -2.166054 & 2.836112 & 0.651808 \\ \mathrm{H} & -1.871703 & 4.548122 & -1.128492 \\ \mathrm{H} & 0.270089 & 4.746879 & -2.353846 \\ \mathrm{H} & -0.494468 & -1.363339 & 0.491029\end{array}$

Figure 12B Radical C4 Product 02

$\begin{array}{lrrr}\text { N } & -5.664623 & -1.505061 & -1.029016 \\ \mathrm{C} & -4.368672 & -2.663947 & 0.790844 \\ \mathrm{C} & -4.419887 & 1.980691 & -1.204769 \\ \mathrm{C} & -4.727947 & 0.539967 & -0.915334 \\ \mathrm{C} & -5.680310 & -0.238864 & -1.561069 \\ \mathrm{C} & -4.712176 & -1.572098 & -0.033050 \\ \mathrm{C} & -4.112972 & -0.302844 & 0.049099 \\ \mathrm{C} & -3.031184 & -0.018188 & 1.054972 \\ \mathrm{C} & -2.730438 & -1.231637 & 1.912520 \\ \mathrm{C} & -3.356860 & -2.441867 & 1.760379 \\ \mathrm{H} & -6.267628 & -2.263727 & -1.311314 \\ \mathrm{H} & -4.852887 & -3.629634 & 0.690682 \\ \mathrm{H} & -3.409096 & 2.105693 & -1.612315 \\ \mathrm{H} & -5.126640 & 2.395317 & -1.930903 \\ \mathrm{H} & -6.361902 & 0.021451 & -2.358742 \\ \mathrm{H} & -3.345675 & 0.811811 & 1.705652 \\ \mathrm{H} & -1.956314 & -1.103708 & 2.664113 \\ \mathrm{H} & -3.068601 & -3.267153 & 2.407145 \\ \mathrm{H} & -4.474801 & 2.596057 & -0.297900 \\ \mathrm{~N} & -1.789741 & 0.484416 & 0.412421 \\ \mathrm{C} & 3.393620 & 0.344507 & -1.347710 \\ \mathrm{O} & 3.465095 & 1.496649 & -1.784438 \\ \mathrm{C} & 2.231320 & -0.602238 & -1.704169 \\ \mathrm{C} & -1.223812 & 2.477230 & 1.864067 \\ \mathrm{C} & 4.332610 & -1.601017 & -0.094206 \\ \mathrm{C} & 5.275344 & -1.562910 & 1.114369 \\ \mathrm{C} & 6.320372 & -0.511983 & 0.698067 \\ \mathrm{C} & 5.511263 & 0.544794 & -0.076421 \\ \mathrm{C} & 2.914950 & -2.080519 & 0.201039 \\ \mathrm{C} & 0.933324 & 0.153318 & -2.095743\end{array}$




$\begin{array}{rrrr}\mathrm{C} & 0.014689 & 0.537153 & -0.967951 \\ \mathrm{C} & -1.152935 & -0.120099 & -0.652716 \\ \mathrm{C} & -1.017638 & 1.556089 & 0.826795 \\ \mathrm{C} & 0.124026 & 1.627022 & -0.026285 \\ \mathrm{C} & 1.056577 & 2.663538 & 0.166518 \\ \mathrm{C} & 0.846140 & 3.580391 & 1.189606 \\ \mathrm{C} & -0.281156 & 3.485790 & 2.031692 \\ \mathrm{~N} & 1.978813 & -1.561253 & -0.629573 \\ \mathrm{~N} & 4.344965 & -0.206656 & -0.567627 \\ \mathrm{O} & 2.663851 & -2.878057 & 1.101978 \\ \mathrm{H} & 2.565680 & -1.141356 & -2.605725 \\ \mathrm{H} & -2.085993 & 2.413384 & 2.521170 \\ \mathrm{H} & 4.749977 & -2.261047 & -0.872583 \\ \mathrm{H} & 5.706895 & -2.542765 & 1.329361 \\ \mathrm{H} & 4.719080 & -1.236283 & 1.999369 \\ \mathrm{H} & 7.068926 & -0.966963 & 0.039579 \\ \mathrm{H} & 6.847681 & -0.078715 & 1.551943 \\ \mathrm{H} & 6.062741 & 0.986029 & -0.912011 \\ \mathrm{H} & 5.169527 & 1.363459 & 0.567636 \\ \mathrm{H} & 0.378782 & -0.488271 & -2.789357 \\ \mathrm{H} & 1.255473 & 1.033307 & -2.661079 \\ \mathrm{H} & -1.609187 & -0.973697 & -1.136602 \\ \mathrm{H} & 1.930300 & 2.733343 & -0.473805 \\ \mathrm{H} & 1.561889 & 4.383038 & 1.347257 \\ \mathrm{H} & -0.417801 & 4.212953 & 2.827751 \\ \mathrm{H} & 1.012613 & -1.785057 & -0.416345\end{array}$

Figure 12B Radical C5 TS

$\begin{array}{lrrr}02 & & & \\ \mathrm{~N} & 2.236957 & -2.686931 & -0.632466 \\ \mathrm{C} & 1.662316 & -0.537246 & -1.806753 \\ \mathrm{C} & 5.349938 & -2.070473 & 1.316957 \\ \mathrm{C} & 4.115946 & -2.049460 & 0.466848 \\ \mathrm{C} & 3.252491 & -3.087456 & 0.228532 \\ \mathrm{C} & 2.410568 & -1.366402 & -0.937084 \\ \mathrm{C} & 3.581404 & -0.909011 & -0.254254 \\ \mathrm{C} & 3.937526 & 0.431557 & -0.348215 \\ \mathrm{C} & 3.039670 & 1.346157 & -1.018425 \\ \mathrm{C} & 2.022659 & 0.780644 & -1.898485 \\ \mathrm{H} & 1.350145 & -3.163662 & -0.785830 \\ \mathrm{H} & 0.843812 & -0.948408 & -2.389519 \\ \mathrm{H} & 5.305035 & -1.313997 & 2.111241 \\ \mathrm{H} & 5.483653 & -3.047084 & 1.793139 \\ \mathrm{H} & 3.277292 & -4.101529 & 0.603968 \\ \mathrm{H} & 4.811525 & 0.825544 & 0.162060 \\ \mathrm{H} & 3.469106 & 2.294703 & -1.325653 \\ \mathrm{H} & 1.481145 & 1.452229 & -2.555576 \\ \mathrm{H} & 6.253039 & -1.860268 & 0.728982 \\ \mathrm{~N} & 1.960608 & 2.109903 & 0.357242 \\ \mathrm{C} & -2.974004 & -0.093844 & 1.145800 \\ \mathrm{O} & -3.541887 & 0.951474 & 1.467915 \\ \mathrm{C} & -1.644284 & -0.545738 & 1.777681 \\ \mathrm{C} & 0.930305 & 3.987277 & -0.977513 \\ \mathrm{C} & -2.883660 & -2.268070 & -0.080182 \\ \mathrm{C} & -3.515865 & -2.602079 & -1.437524\end{array}$

$\begin{array}{rrrr}\mathrm{C} & -4.929010 & -2.004766 & -1.309200 \\ \mathrm{C} & -4.724587 & -0.710885 & -0.499341 \\ \mathrm{C} & -1.361511 & -2.200277 & -0.072351 \\ \mathrm{C} & -0.815116 & 0.622665 & 2.374895 \\ \mathrm{C} & 0.046884 & 1.399294 & 1.417846 \\ \mathrm{C} & 1.397278 & 1.193408 & 1.220057 \\ \mathrm{C} & 0.903479 & 2.914969 & -0.080944 \\ \mathrm{C} & -0.301627 & 2.526955 & 0.574555 \\ \mathrm{C} & -1.481075 & 3.250658 & 0.341551 \\ \mathrm{C} & -1.448412 & 4.318537 & -0.554812 \\ \mathrm{C} & -0.257617 & 4.680861 & -1.211021 \\ \mathrm{~N} & -0.852886 & -1.333306 & 0.826877 \\ \mathrm{~N} & -3.486179 & -0.967808 & 0.253559 \\ \mathrm{O} & -0.680593 & -2.901086 & -0.831779 \\ \mathrm{H} & -1.928352 & -1.190209 & 2.625048 \\ \mathrm{H} & 1.851388 & 4.277271 & -1.476651 \\ \mathrm{H} & -3.183297 & -3.022686 & 0.666032 \\ \mathrm{H} & -3.514204 & -3.675363 & -1.638395 \\ \mathrm{H} & -2.951180 & -2.106398 & -2.234258 \\ \mathrm{H} & -5.578810 & -2.693502 & -0.757736 \\ \mathrm{H} & -5.394798 & -1.811538 & -2.278889 \\ \mathrm{H} & -5.550542 & -0.493560 & 0.184413 \\ \mathrm{H} & -4.582697 & 0.166053 & -1.141459 \\ \mathrm{H} & -0.173164 & 0.192527 & 3.152049 \\ \mathrm{H} & -1.535604 & 1.273704 & 2.879268 \\ \mathrm{H} & 2.026523 & 0.448653 & 1.692590 \\ \mathrm{H} & -2.402677 & 2.968679 & 0.841261 \\ \mathrm{H} & -2.356996 & 4.883006 & -0.749056 \\ \mathrm{H} & -0.260697 & 5.517253 & -1.905227 \\ \mathrm{H} & 0.148515 & -1.171179 & 0.800912 \\ & & & \end{array}$

Figure 12B Radical C5 Product

02

$\begin{array}{lrrr}\text { N } & 5.506655 & -2.301124 & -0.650018 \\ \mathrm{C} & 3.545351 & -1.266604 & -1.878345 \\ \mathrm{C} & 6.429878 & 0.039847 & 2.085015 \\ \mathrm{C} & 5.862558 & -0.717985 & 0.923464 \\ \mathrm{C} & 6.285557 & -1.928739 & 0.435252 \\ \mathrm{C} & 4.563612 & -1.333758 & -0.884671 \\ \mathrm{C} & 4.748456 & -0.308469 & 0.088363 \\ \mathrm{C} & 3.925819 & 0.810659 & 0.093502 \\ \mathrm{C} & 2.814537 & 0.958433 & -0.916385 \\ \mathrm{C} & 2.723145 & -0.186013 & -1.907288 \\ \mathrm{H} & 5.622609 & -3.151216 & -1.181692 \\ \mathrm{H} & 3.430391 & -2.069815 & -2.602325 \\ \mathrm{H} & 5.677161 & 0.207157 & 2.866920 \\ \mathrm{H} & 7.266291 & -0.501085 & 2.539509 \\ \mathrm{H} & 7.090237 & -2.567059 & 0.773611 \\ \mathrm{H} & 4.047396 & 1.608546 & 0.820172 \\ \mathrm{H} & 2.960071 & 1.897331 & -1.473934 \\ \mathrm{H} & 1.936779 & -0.111247 & -2.653080 \\ \mathrm{H} & 6.797690 & 1.029614 & 1.783326 \\ \mathrm{~N} & 1.503990 & 1.157945 & -0.238449 \\ \mathrm{C} & -3.558733 & -0.109837 & 1.391811 \\ \mathrm{O} & -3.835493 & 0.941624 & 1.975884\end{array}$




$\begin{array}{lrrr}\text { C } & -2.247248 & -0.875291 & 1.651735 \\ \mathrm{C} & 0.595669 & 3.201242 & -1.421947 \\ \mathrm{C} & -4.136018 & -1.997886 & -0.139140 \\ \mathrm{C} & -5.056816 & -1.949772 & -1.364346 \\ \mathrm{C} & -6.273965 & -1.162691 & -0.845196 \\ \mathrm{C} & -5.671872 & -0.106857 & 0.100799 \\ \mathrm{C} & -2.654085 & -2.185315 & -0.447633 \\ \mathrm{C} & -1.104437 & 0.042465 & 2.163789 \\ \mathrm{C} & -0.271165 & 0.722511 & 1.112251 \\ \mathrm{C} & 0.989247 & 0.321244 & 0.731795 \\ \mathrm{C} & 0.558185 & 2.129579 & -0.518074 \\ \mathrm{C} & -0.572571 & 1.891435 & 0.319406 \\ \mathrm{C} & -1.671223 & 2.768703 & 0.248509 \\ \mathrm{C} & -1.628937 & 3.834126 & -0.642792 \\ \mathrm{C} & -0.507461 & 4.046295 & -1.471375 \\ \mathrm{~N} & -1.827909 & -1.628994 & 0.470768 \\ \mathrm{~N} & -4.394810 & -0.706843 & 0.519015 \\ \mathrm{O} & -2.261537 & -2.803357 & -1.435163 \\ \mathrm{H} & -2.480053 & -1.575876 & 2.470192 \\ \mathrm{H} & 1.452498 & 3.374983 & -2.066152 \\ \mathrm{H} & -4.442412 & -2.822146 & 0.526025 \\ \mathrm{H} & -5.308627 & -2.948160 & -1.728207 \\ \mathrm{H} & -4.555490 & -1.410372 & -2.174982 \\ \mathrm{H} & -6.941639 & -1.828766 & -0.287101 \\ \mathrm{H} & -6.856379 & -0.706389 & -1.649864 \\ \mathrm{H} & -6.300724 & 0.102934 & 0.971260 \\ \mathrm{H} & -5.475340 & 0.845076 & -0.406007 \\ \mathrm{H} & -0.443711 & -0.573112 & 2.784064 \\ \mathrm{H} & -1.578175 & 0.772816 & 2.826931 \\ \mathrm{H} & 1.589154 & -0.497945 & 1.106271 \\ \mathrm{H} & -2.539446 & 2.602706 & 0.878637 \\ \mathrm{H} & -2.473186 & 4.515819 & -0.705582 \\ \mathrm{H} & -0.502968 & 4.885061 & -2.162460 \\ \mathrm{H} & -0.836276 & -1.655808 & 0.258488\end{array}$

Figure 12B Radical C6 TS 02

$\begin{array}{rrrr}N & 1.628868 & 2.371470 & 0.385167 \\ C & -3.057800 & -0.383152 & 1.292029 \\ O & -3.737536 & 0.514829 & 1.793887 \\ C & -1.622581 & -0.692437 & 1.759793 \\ C & 0.332875 & 3.860637 & -1.181784 \\ C & -2.761023 & -2.328535 & -0.245852 \\ C & -3.469395 & -2.577941 & -1.583383 \\ C & -4.936073 & -2.242684 & -1.256950 \\ C & -4.846998 & -1.048538 & -0.289576 \\ C & -1.277190 & -2.000168 & -0.342929 \\ C & -0.921317 & 0.532525 & 2.415086 \\ C & -0.165289 & 1.417839 & 1.465689 \\ C & 1.205666 & 1.451723 & 1.325502 \\ C & 0.465431 & 2.904003 & -0.169023 \\ C & -0.678346 & 2.370905 & 0.497738 \\ C & -1.957553 & 2.836098 & 0.159862 \\ C & -2.081661 & 3.786109 & -0.854961 \\ C & -0.950282 & 4.288995 & -1.522441\end{array}$

$\begin{array}{rrrr}\mathrm{N} & -0.813341 & -1.233708 & 0.663127 \\ \mathrm{~N} & -3.519814 & -1.205633 & 0.327785 \\ \mathrm{O} & -0.579786 & -2.430606 & -1.271574 \\ \mathrm{H} & -1.728718 & -1.460371 & 2.541819 \\ \mathrm{H} & 1.205318 & 4.268784 & -1.685649 \\ \mathrm{H} & -2.859558 & -3.216158 & 0.400716 \\ \mathrm{H} & -3.326397 & -3.600416 & -1.939099 \\ \mathrm{H} & -3.069333 & -1.895647 & -2.340699 \\ \mathrm{H} & -5.413539 & -3.093734 & -0.758530 \\ \mathrm{H} & -5.523112 & -2.005611 & -2.147886 \\ \mathrm{H} & -5.627882 & -1.051486 & 0.476472 \\ \mathrm{H} & -4.892440 & -0.084966 & -0.810284 \\ \mathrm{H} & -0.223341 & 0.150971 & 3.168011 \\ \mathrm{H} & -1.706800 & 1.077960 & 2.947712 \\ \mathrm{H} & 1.941598 & 0.872288 & 1.872365 \\ \mathrm{H} & -2.834709 & 2.460448 & 0.678417 \\ \mathrm{H} & -3.068542 & 4.149615 & -1.130270 \\ \mathrm{H} & -1.075982 & 5.029132 & -2.308464 \\ \mathrm{H} & 0.141219 & -0.893382 & 0.590969 \\ \mathrm{~N} & 2.283452 & -2.063674 & -1.021218 \\ \mathrm{C} & 2.276500 & 0.423216 & -1.356496 \\ \mathrm{C} & 5.321341 & -2.829177 & 0.988720 \\ \mathrm{C} & 4.184181 & -2.233125 & 0.212777 \\ \mathrm{C} & 3.120222 & -2.922849 & -0.355244 \\ \mathrm{C} & 2.766582 & -0.773905 & -0.864981 \\ \mathrm{C} & 3.968233 & -0.860003 & -0.091867 \\ \mathrm{C} & 4.673715 & 0.341163 & 0.210390 \\ \mathrm{C} & 4.167951 & 1.544587 & -0.201453 \\ \mathrm{C} & 2.904802 & 1.638663 & -0.916648 \\ \mathrm{H} & 1.322837 & -2.282504 & -1.301820 \\ \mathrm{H} & 1.389518 & 0.466976 & -1.980630 \\ \mathrm{H} & 5.196008 & -3.910690 & 1.101922 \\ \mathrm{H} & 6.285879 & -2.656927 & 0.493982 \\ \mathrm{H} & 2.906609 & -3.983242 & -0.326546 \\ \mathrm{H} & 5.612699 & 0.297331 & 0.756450 \\ \mathrm{H} & 4.690834 & 2.468077 & 0.027645 \\ \mathrm{H} & 2.774659 & 2.511858 & -1.546851 \\ \mathrm{H} & 5.395658 & -2.394980 & 1.993737 \\ & & & \end{array}$

Figure 12B Radical C6 Product 02

$\begin{array}{rrrr}\mathrm{N} & 1.436848 & 1.622912 & 0.112574 \\ \mathrm{C} & -3.604476 & -0.172117 & 1.222682 \\ \mathrm{O} & -4.097955 & 0.886551 & 1.621131 \\ \mathrm{C} & -2.255581 & -0.702578 & 1.745995 \\ \mathrm{C} & 0.459894 & 3.367009 & -1.436960 \\ \mathrm{C} & -3.697515 & -2.288584 & -0.099461 \\ \mathrm{C} & -4.442957 & -2.532692 & -1.416833 \\ \mathrm{C} & -5.814839 & -1.883871 & -1.157035 \\ \mathrm{C} & -5.492909 & -0.637326 & -0.312355 \\ \mathrm{C} & -2.175687 & -2.273281 & -0.205508 \\ \mathrm{C} & -1.331034 & 0.422865 & 2.284649 \\ \mathrm{C} & -0.445533 & 1.096732 & 1.272594 \\ \mathrm{C} & 0.895642 & 0.834058 & 1.108268 \\ \mathrm{C} & 0.426845 & 2.412550 & -0.410078\end{array}$




$\begin{array}{rrrr}\mathrm{C} & -0.772964 & 2.115816 & 0.302999 \\ \mathrm{C} & -1.950070 & 2.817288 & -0.019682 \\ \mathrm{C} & -1.913237 & 3.768918 & -1.031877 \\ \mathrm{C} & -0.720741 & 4.038381 & -1.735338 \\ \mathrm{~N} & -1.571193 & -1.505695 & 0.733230 \\ \mathrm{~N} & -4.219617 & -0.983465 & 0.338954 \\ \mathrm{O} & -1.567735 & -2.916477 & -1.058735 \\ \mathrm{H} & -2.511187 & -1.340614 & 2.607608 \\ \mathrm{H} & 1.371383 & 3.582601 & -1.986652 \\ \mathrm{H} & -3.971852 & -3.064270 & 0.634448 \\ \mathrm{H} & -4.504921 & -3.594936 & -1.662792 \\ \mathrm{H} & -3.915578 & -2.026417 & -2.232287 \\ \mathrm{H} & -6.451545 & -2.568739 & -0.585669 \\ \mathrm{H} & -6.343498 & -1.628614 & -2.079131 \\ \mathrm{H} & -6.257677 & -0.411617 & 0.436791 \\ \mathrm{H} & -5.353255 & 0.259258 & -0.927638 \\ \mathrm{H} & -0.695921 & -0.019308 & 3.060005 \\ \mathrm{H} & -1.988623 & 1.143410 & 2.780611 \\ \mathrm{H} & 1.532551 & 0.152188 & 1.656451 \\ \mathrm{H} & -2.872099 & 2.603165 & 0.511838 \\ \mathrm{H} & -2.817433 & 4.314260 & -1.289613 \\ \mathrm{H} & -0.722161 & 4.784083 & -2.525947 \\ \mathrm{H} & -0.568118 & -1.385495 & 0.639808 \\ \mathrm{~N} & 4.218573 & -1.871805 & -1.583379 \\ \mathrm{C} & 2.976331 & 0.276843 & -1.272523 \\ \mathrm{C} & 6.743454 & -2.045623 & 1.138391 \\ \mathrm{C} & 5.649742 & -1.716506 & 0.164408 \\ \mathrm{C} & 5.249077 & -2.495512 & -0.912561 \\ \mathrm{C} & 3.921427 & -0.670948 & -0.956805 \\ \mathrm{C} & 4.822370 & -0.561871 & 0.153096 \\ \mathrm{C} & 4.734396 & 0.597223 & 0.994207 \\ \mathrm{C} & 3.815143 & 1.562243 & 0.747877 \\ \mathrm{C} & 2.828369 & 1.500281 & -0.399410 \\ \mathrm{H} & 3.747171 & -2.238856 & -2.396416 \\ \mathrm{H} & 2.305881 & 0.170741 & -2.120004 \\ \mathrm{H} & 6.366437 & -2.104350 & 2.167401 \\ \mathrm{H} & 7.206416 & -3.008473 & 0.899794 \\ \mathrm{H} & 5.628546 & -3.450553 & -1.248835 \\ \mathrm{H} & 5.416107 & 0.701721 & 1.835383 \\ \mathrm{H} & 3.746935 & 2.440518 & 1.384119 \\ \mathrm{H} & 2.959564 & 2.405961 & -1.011627 \\ \mathrm{H} & 7.535235 & -1.285673 & 1.131104\end{array}$

Figure 12B Radical C7 TS

02

$\begin{array}{lrrr}\text { N } & -2.628234 & -0.497297 & 1.886417 \\ \mathrm{C} & -3.844699 & 0.303962 & -0.181696 \\ \mathrm{C} & -1.497132 & -3.974606 & 1.227141 \\ \mathrm{C} & -2.069454 & -2.589328 & 1.243453 \\ \mathrm{C} & -1.976295 & -1.662915 & 2.253534 \\ \mathrm{C} & -3.143138 & -0.642738 & 0.628298 \\ \mathrm{C} & -2.815957 & -1.949175 & 0.186024 \\ \mathrm{C} & -3.275365 & -2.379946 & -1.071805 \\ \mathrm{C} & -4.066239 & -1.507232 & -1.845761 \\ \mathrm{C} & -4.383104 & -0.230805 & -1.405833\end{array}$

$\begin{array}{rrrr}\mathrm{H} & -2.617827 & 0.364504 & 2.414395 \\ \mathrm{H} & -4.382883 & 1.112494 & 0.299795 \\ \mathrm{H} & -0.659403 & -4.040840 & 0.523207 \\ \mathrm{H} & -1.121136 & -4.254568 & 2.216604 \\ \mathrm{H} & -1.502167 & -1.740486 & 3.222042 \\ \mathrm{H} & -3.048219 & -3.378542 & -1.433994 \\ \mathrm{H} & -4.457837 & -1.853008 & -2.798484 \\ \mathrm{H} & -5.008589 & 0.416078 & -2.013023 \\ \mathrm{H} & -2.246966 & -4.718987 & 0.931414 \\ \mathrm{~N} & -2.461876 & 1.623527 & -0.803679 \\ \mathrm{C} & 3.084488 & 0.894715 & -0.916491 \\ \mathrm{O} & 3.347774 & 2.098162 & -0.850916 \\ \mathrm{C} & 1.999625 & 0.323760 & -1.850563 \\ \mathrm{C} & -2.217282 & 2.895311 & 1.356540 \\ \mathrm{C} & 3.508165 & -1.494246 & -0.344652 \\ \mathrm{C} & 4.132065 & -2.061124 & 0.936776 \\ \mathrm{C} & 5.323878 & -1.118183 & 1.182846 \\ \mathrm{C} & 4.807108 & 0.267435 & 0.755765 \\ \mathrm{C} & 2.023053 & -1.795283 & -0.534278 \\ \mathrm{C} & 0.950620 & 1.375452 & -2.292540 \\ \mathrm{C} & -0.244749 & 1.572437 & -1.401017 \\ \mathrm{C} & -1.527179 & 1.155518 & -1.699769 \\ \mathrm{C} & -1.744752 & 2.276043 & 0.192721 \\ \mathrm{C} & -0.358873 & 2.307834 & -0.153470 \\ \mathrm{C} & 0.540471 & 3.006610 & 0.663752 \\ \mathrm{C} & 0.064016 & 3.612645 & 1.828252 \\ \mathrm{C} & -1.297171 & 3.553547 & 2.175311 \\ \mathrm{~N} & 1.367041 & -0.852556 & -1.251847 \\ \mathrm{~N} & 3.753313 & -0.047396 & -0.221174 \\ \mathrm{O} & 1.499291 & -2.810137 & -0.077969 \\ \mathrm{H} & 2.538657 & 0.019676 & -2.762982 \\ \mathrm{H} & -3.274923 & 2.884547 & 1.609841 \\ \mathrm{H} & 4.041094 & -1.887636 & -1.226202 \\ \mathrm{H} & 4.423352 & -3.107441 & 0.823008 \\ \mathrm{H} & 3.405132 & -1.999420 & 1.753508 \\ \mathrm{H} & 6.170831 & -1.412135 & 0.552616 \\ \mathrm{H} & 5.662838 & -1.127101 & 2.222048 \\ \mathrm{H} & 5.576956 & 0.896452 & 0.299105 \\ \mathrm{H} & 4.372567 & 0.826083 & 1.593241 \\ \mathrm{H} & 0.589209 & 1.076258 & -3.282398 \\ \mathrm{H} & 1.503684 & 2.311553 & -2.423163 \\ \mathrm{H} & -1.851245 & 0.582232 & -2.561182 \\ \mathrm{H} & 1.590288 & 3.070812 & 0.393925 \\ \mathrm{H} & 0.755801 & 4.147051 & 2.474397 \\ \mathrm{H} & -1.641530 & 4.038742 & 3.084861 \\ & 0.361500 & -0.952577 & -1.344759\end{array}$

Figure 12B Radical C7 Product

02

$\begin{array}{lrrr}\text { N } & -2.852943 & -0.675510 & 1.620853 \\ \text { C } & -3.219138 & 1.210708 & -0.070840 \\ \text { C } & -4.910853 & -3.600901 & 0.644288 \\ \text { C } & -4.185744 & -2.298677 & 0.815185 \\ \text { C } & -3.298911 & -1.972362 & 1.814342 \\ \text { C } & -3.433299 & -0.159537 & 0.497792\end{array}$




\begin{tabular}{|c|c|c|c|}
\hline & 1 & 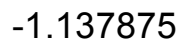 & \\
\hline & 9761 & 34899 & \\
\hline & 8436 & & \\
\hline 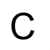 & -4.042682 & 1.380836 & \\
\hline & -2.163471 & -0.208317 & 2.1927 \\
\hline & -3.524420 & 1.979235 & 0.6 \\
\hline & -4.678300 & -4.072443 & -0.3 \\
\hline & -4.637501 & -4.309005 & 1.4 \\
\hline & -2.942706 & -2.557190 & 2.65066 \\
\hline & -5.679523 & -1.609766 & -1.6 \\
\hline & -5.430641 & 0.617392 & -2.73696 \\
\hline & -3.957455 & 2.343463 & -1.82314 \\
\hline & -6.000275 & -3.469381 & 0.67970 \\
\hline & -1.786180 & 046 & -0.32120 \\
\hline & & 283 & \\
\hline & 3.9 & & \\
\hline$\checkmark$ & 2.30 & & \\
\hline 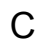 & -1.17 & & \\
\hline c & & & \\
\hline U & & -2.5 & \\
\hline 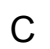 & 5.6 & -1.7 & \\
\hline & 5.2 & & \\
\hline & & & \\
\hline & 1.34 & & -2.2 \\
\hline & 0.27 & & -1 \\
\hline & -1 & & -1 \\
\hline & -0. & & \\
\hline & 0.3 & 55 & -0.0 \\
\hline & & & \\
\hline & 1.1 & & \\
\hline & -0.1 & & 2.3 \\
\hline & & -1.2 & -1.1 \\
\hline $\mathbf{I V}$ & 4.1 & -0.6 & -0.2 \\
\hline & & -3. & 0.2 \\
\hline & 2.72 & -0.5 & -2.7 \\
\hline & -2.17 & & 2.1 \\
\hline & 4.11 & -2.5 & -1.0 \\
\hline & 4.41 & -3.5 & \\
\hline & & -2.2 & 1.9 \\
\hline & & -2.2 & \\
\hline & 493 & -1.6 & 2.1 \\
\hline $\mathrm{H}$ & 6.081441 & & 0.01 \\
\hline $\mathrm{H}$ & 4.960381 & 355 & 1.35082 \\
\hline $\mathrm{H}$ & 0.854010 & 0.706083 & -3.19641 \\
\hline 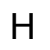 & 1.980598 & 1.858465 & -2.48700 \\
\hline & -1.546984 & 9104 & -2.16759 \\
\hline & 2.446722 & 601 & 0.21 \\
\hline & 2.006028 & 027 & 2.34378 \\
\hline & -0.273527 & & \\
\hline & 0.577978 & -1.206794 & -1.18 \\
\hline
\end{tabular}

Compound SI-6

01

$\begin{array}{llll}\mathrm{N} & -3.411545 & 1.506824 & -0.600124\end{array}$

$\begin{array}{llll}\text { C } & 1.358177 & -0.248240 & 1.359732\end{array}$

$\begin{array}{lrrr}\mathrm{O} & 0.933925 & -1.082974 & 2.164039 \\ \mathrm{C} & 0.823028 & 1.195582 & 1.318693 \\ \mathrm{C} & -3.998417 & -0.847060 & -1.280641 \\ \mathrm{C} & 2.945599 & 0.480348 & -0.427680 \\ \mathrm{C} & 3.579456 & -0.396553 & -1.514122 \\ \mathrm{C} & 4.050463 & -1.629507 & -0.721584 \\ \mathrm{C} & 2.956197 & -1.842449 & 0.341574 \\ \mathrm{C} & 1.912163 & 1.485608 & -0.923507 \\ \mathrm{C} & -0.632012 & 1.312448 & 1.846299 \\ \mathrm{C} & -1.729816 & 1.067741 & 0.847147 \\ \mathrm{C} & -2.486681 & 2.057690 & 0.261036 \\ \mathrm{C} & -3.279619 & 0.134434 & -0.587631 \\ \mathrm{C} & -2.224383 & -0.183751 & 0.315383 \\ \mathrm{C} & -1.900592 & -1.538844 & 0.522785 \\ \mathrm{C} & -2.614339 & -2.515452 & -0.161952 \\ \mathrm{C} & -3.651366 & -2.174342 & -1.056257 \\ \mathrm{~N} & 0.944798 & 1.772342 & -0.019162 \\ \mathrm{~N} & 2.352438 & -0.507877 & 0.487718 \\ \mathrm{O} & 1.976136 & 1.996010 & -2.039887 \\ \mathrm{H} & -4.090602 & 2.027075 & -1.135132 \\ \mathrm{H} & 1.458503 & 1.758574 & 2.021697 \\ \mathrm{H} & -4.798876 & -0.579752 & -1.965306 \\ \mathrm{H} & 3.731268 & 1.048551 & 0.097580 \\ \mathrm{H} & 4.387958 & 0.117843 & -2.037783 \\ \mathrm{H} & 2.817025 & -0.669696 & -2.251358 \\ \mathrm{H} & 5.009386 & -1.418559 & -0.234994 \\ \mathrm{H} & 4.184679 & -2.511838 & -1.352667 \\ \mathrm{H} & 3.348472 & -2.193769 & 1.300695 \\ \mathrm{H} & 2.187887 & -2.552612 & 0.014878 \\ \mathrm{H} & -0.752821 & 2.323525 & 2.250832 \\ \mathrm{H} & -0.703840 & 0.619364 & 2.690162 \\ \mathrm{H} & -2.448788 & 3.128955 & 0.409946 \\ \mathrm{H} & -1.099900 & -1.808675 & 1.204075 \\ \mathrm{H} & -2.371032 & -3.563439 & -0.007429 \\ \mathrm{H} & -4.189824 & -2.961729 & -1.576910 \\ \mathrm{H} & 0.203031 & 2.386955 & -0.336186\end{array}$

\section{Compound SI-PC-I}

04

$\begin{array}{lrrr}\mathrm{H} & 0.156796 & 0.114742 & 0.205381 \\ \mathrm{Fe} & -2.998491 & -0.235894 & 0.008038 \\ \mathrm{~N} & -3.474594 & -2.190562 & 0.184175 \\ \mathrm{~N} & -2.222544 & -0.595080 & -1.817988 \\ \mathrm{~N} & -4.078481 & 0.149371 & 1.666905 \\ \mathrm{~N} & -2.792431 & 1.742806 & -0.306468 \\ \mathrm{C} & -4.067139 & -2.802783 & 1.259344 \\ \mathrm{C} & -1.642011 & 0.318113 & -2.660525 \\ \mathrm{C} & -3.139187 & -3.201730 & -0.686097 \\ \mathrm{C} & -2.059578 & -1.817107 & -2.418291 \\ \mathrm{C} & -4.604047 & -0.776450 & 2.547968 \\ \mathrm{C} & -2.123730 & 2.346625 & -1.347260 \\ \mathrm{C} & -4.288463 & 1.381361 & 2.249504 \\ \mathrm{C} & -3.150039 & 2.755235 & 0.545957 \\ \mathrm{C} & -4.110441 & -4.231010 & 1.063532 \\ \mathrm{C} & -1.101164 & -0.342825 & -3.821983\end{array}$




\begin{tabular}{|c|c|c|c|}
\hline & & & \\
\hline & & & \\
\hline & 734 & & \\
\hline & 7299 & 025 & \\
\hline & 367 & 283 & \\
\hline & -2.705897 & 4.027305 & \\
\hline & 443 & & \\
\hline & & 9842 & \\
\hline & 086 & & \\
\hline & 095 & & \\
\hline & -5.65 & & \\
\hline & -1.597885 & & \\
\hline & & & \\
\hline & & & \\
\hline & & & \\
\hline & & & \\
\hline & & & \\
\hline & & & \\
\hline & & & \\
\hline & & & \\
\hline & & & \\
\hline & & & \\
\hline & & & \\
\hline & & & \\
\hline & & & \\
\hline & & & \\
\hline & & & \\
\hline & & & \\
\hline & & & \\
\hline & & & \\
\hline & & & \\
\hline & & & \\
\hline & & & \\
\hline & & & \\
\hline & & & \\
\hline & & & \\
\hline & & & \\
\hline & & & \\
\hline & & & \\
\hline & & & \\
\hline & & & \\
\hline & & & \\
\hline & & & \\
\hline & & 2.5 & \\
\hline & & & \\
\hline & & & \\
\hline & & & \\
\hline & & & \\
\hline & & & \\
\hline & & & \\
\hline & & & \\
\hline & & & \\
\hline & & & \\
\hline & & & \\
\hline & 67 & & \\
\hline
\end{tabular}

$\begin{array}{rrrr}\mathrm{H} & 9.382792 & 0.244539 & 2.650529 \\ \mathrm{H} & 9.060415 & 0.790638 & -0.073253 \\ \mathrm{H} & 7.921044 & 1.424830 & 1.122308 \\ \mathrm{H} & 3.796240 & -1.179573 & -2.606032 \\ \mathrm{H} & 4.649350 & 0.361091 & -2.632839 \\ \mathrm{H} & 1.536536 & -1.383130 & -1.264351 \\ \mathrm{H} & 4.926492 & 2.500548 & -0.744185 \\ \mathrm{H} & 4.229274 & 4.465391 & 0.605162 \\ \mathrm{H} & 2.007562 & 4.533431 & 1.698896 \\ \mathrm{H} & 3.969441 & -1.937277 & 0.061203\end{array}$

TS from SI-PC-I to SI-R-I

04

$\begin{array}{lrrr}\mathrm{H} & 0.212881 & -0.095237 & 0.260839 \\ \mathrm{Fe} & 2.755812 & 0.248505 & -0.018954 \\ \mathrm{~N} & 3.073813 & 2.234834 & 0.209025 \\ \mathrm{~N} & 1.894495 & 0.612618 & -1.814512 \\ \mathrm{~N} & 3.745924 & -0.115029 & 1.701589 \\ \mathrm{~N} & 2.591814 & -1.733687 & -0.333383 \\ \mathrm{C} & 3.705567 & 2.843470 & 1.261672 \\ \mathrm{C} & 1.440768 & -0.323620 & -2.707577 \\ \mathrm{C} & 2.632885 & 3.241688 & -0.610806 \\ \mathrm{C} & 1.613894 & 1.837898 & -2.362039 \\ \mathrm{C} & 4.277824 & 0.820746 & 2.552374 \\ \mathrm{C} & 2.036436 & -2.347116 & -1.426516 \\ \mathrm{C} & 3.930599 & -1.332468 & 2.303013 \\ \mathrm{C} & 2.932328 & -2.736413 & 0.541210 \\ \mathrm{C} & 3.673873 & 4.278390 & 1.096538 \\ \mathrm{C} & 0.852443 & 0.329265 & -3.852857 \\ \mathrm{C} & 3.004603 & 4.525599 & -0.062788 \\ \mathrm{C} & 0.954884 & 1.671067 & -3.635923 \\ \mathrm{C} & 4.827519 & 0.171359 & 3.719975 \\ \mathrm{C} & 2.039686 & -3.779304 & -1.245453 \\ \mathrm{C} & 4.607783 & -1.163546 & 3.567994 \\ \mathrm{C} & 2.588500 & -4.020393 & -0.022055 \\ \mathrm{H} & 4.107686 & 4.986413 & 1.791029 \\ \mathrm{H} & 0.423745 & -0.187245 & -4.702229 \\ \mathrm{H} & 2.776470 & 5.478977 & -0.522069 \\ \mathrm{H} & 0.632488 & 2.485499 & -4.272199 \\ \mathrm{H} & 5.309591 & 0.688136 & 4.540018 \\ \mathrm{H} & 1.661069 & -4.490757 & -1.968216 \\ \mathrm{H} & 4.874204 & -1.973242 & 4.235320 \\ \mathrm{H} & 2.758950 & -4.971185 & 0.466712 \\ \mathrm{C} & 4.273953 & 2.194235 & 2.350870 \\ \mathrm{C} & 1.509632 & -1.700771 & -2.539342 \\ \mathrm{C} & 3.542441 & -2.559050 & 1.776030 \\ \mathrm{C} & 1.942814 & 3.067812 & -1.803464 \\ \mathrm{H} & 4.735618 & 2.811822 & 3.114675 \\ \mathrm{H} & 1.103629 & -2.322119 & -3.331271 \\ \mathrm{H} & 3.754107 & -3.447071 & 2.362995 \\ \mathrm{H} & 1.659466 & 3.959674 & -2.353184 \\ \mathrm{O} & 1.258203 & 0.321697 & 0.786757 \\ \mathrm{~S} & 4.875395 & 0.265719 & -1.175643 \\ \mathrm{C} & 5.781432 & -1.282407 & -0.802588 \\ \mathrm{H} & 5.235575 & -2.161776 & -1.154976\end{array}$




$\begin{array}{rrrr}\mathrm{H} & 6.745971 & -1.236476 & -1.318420 \\ \mathrm{H} & 5.964774 & -1.386762 & 0.270695 \\ \mathrm{~N} & -0.886881 & -0.475613 & -0.161706 \\ \mathrm{C} & -6.100032 & -0.177158 & -0.646909 \\ \mathrm{O} & -6.314447 & -1.326261 & -1.044886 \\ \mathrm{C} & -5.164180 & 0.766650 & -1.422137 \\ \mathrm{C} & -1.090980 & -2.681852 & 1.016169 \\ \mathrm{C} & -6.543099 & 1.722981 & 0.922303 \\ \mathrm{C} & -6.786999 & 1.597455 & 2.431666 \\ \mathrm{C} & -7.867430 & 0.501842 & 2.500588 \\ \mathrm{C} & -7.502421 & -0.476694 & 1.365110 \\ \mathrm{C} & -5.227610 & 2.370521 & 0.520930 \\ \mathrm{C} & -4.015764 & -0.019858 & -2.110630 \\ \mathrm{C} & -2.917520 & -0.496881 & -1.209869 \\ \mathrm{C} & -1.692665 & 0.198096 & -0.975773 \\ \mathrm{C} & -1.540376 & -1.663124 & 0.195092 \\ \mathrm{C} & -2.812636 & -1.706251 & -0.446575 \\ \mathrm{C} & -3.644998 & -2.822720 & -0.252236 \\ \mathrm{C} & -3.193245 & -3.858540 & 0.575941 \\ \mathrm{C} & -1.942584 & -3.791203 & 1.199523 \\ \mathrm{~N} & -4.631103 & 1.831615 & -0.575473 \\ \mathrm{~N} & -6.673486 & 0.336052 & 0.456742 \\ \mathrm{O} & -4.769363 & 3.330673 & 1.132889 \\ \mathrm{H} & -5.771496 & 1.197180 & -2.233894 \\ \mathrm{H} & -0.119183 & -2.633828 & 1.497020 \\ \mathrm{H} & -7.342078 & 2.337331 & 0.475455 \\ \mathrm{H} & -7.098529 & 2.544054 & 2.877360 \\ \mathrm{H} & -5.864761 & 1.272752 & 2.925922 \\ \mathrm{H} & -8.854930 & 0.938959 & 2.315968 \\ \mathrm{H} & -7.900919 & 0.003236 & 3.472560 \\ \mathrm{H} & -8.377817 & -0.870731 & 0.839929 \\ \mathrm{H} & -6.911801 & -1.329618 & 1.716414 \\ \mathrm{H} & -3.585814 & 0.632455 & -2.879681 \\ \mathrm{H} & -4.486989 & -0.864366 & -2.620719 \\ \mathrm{H} & -1.384139 & 1.145522 & -1.401398 \\ \mathrm{H} & -4.623661 & -2.864105 & -0.717478 \\ \mathrm{H} & -3.825404 & -4.726403 & 0.737604 \\ \mathrm{H} & -1.616231 & -4.606958 & 1.837732 \\ \mathrm{H} & -3.811043 & 2.330622 & -0.902223\end{array}$

Compound SI-R-I

02

$\begin{array}{rrrr}\text { N } & -3.049957 & 1.767668 & -0.938990 \\ \mathrm{C} & 1.226975 & -0.379821 & 1.350606 \\ \mathrm{O} & 0.668817 & -1.205515 & 2.078688 \\ \mathrm{C} & 0.891815 & 1.119025 & 1.446887 \\ \mathrm{C} & -3.937156 & -0.528659 & -1.404071 \\ \mathrm{C} & 2.951727 & 0.232762 & -0.356465 \\ \mathrm{C} & 3.358945 & -0.614142 & -1.568735 \\ \mathrm{C} & 3.598159 & -1.999161 & -0.939197 \\ \mathrm{C} & 2.528685 & -2.114275 & 0.165686 \\ \mathrm{C} & 2.191476 & 1.510604 & -0.674993 \\ \mathrm{C} & -0.590898 & 1.344209 & 1.848498 \\ \mathrm{C} & -1.600186 & 1.142981 & 0.761433 \\ \mathrm{C} & -2.163567 & 2.189106 & -0.058441\end{array}$

$\begin{array}{rrrr}\mathrm{C} & -3.128193 & 0.370188 & -0.735631 \\ \mathrm{C} & -2.247992 & -0.055782 & 0.304737 \\ \mathrm{C} & -2.194798 & -1.406082 & 0.671805 \\ \mathrm{C} & -3.019767 & -2.316515 & -0.008681 \\ \mathrm{C} & -3.873622 & -1.888708 & -1.027255 \\ \mathrm{~N} & 1.213738 & 1.831963 & 0.211707 \\ \mathrm{~N} & 2.173231 & -0.713534 & 0.453780 \\ \mathrm{O} & 2.474247 & 2.204186 & -1.647704 \\ \mathrm{H} & 1.499014 & 1.511817 & 2.277799 \\ \mathrm{H} & -4.604721 & -0.196304 & -2.193602 \\ \mathrm{H} & 3.855768 & 0.535333 & 0.197462 \\ \mathrm{H} & 4.236716 & -0.209331 & -2.076008 \\ \mathrm{H} & 2.532376 & -0.644972 & -2.286978 \\ \mathrm{H} & 4.599817 & -2.040306 & -0.497273 \\ \mathrm{H} & 3.522188 & -2.811683 & -1.666205 \\ \mathrm{H} & 2.895304 & -2.612983 & 1.068029 \\ \mathrm{H} & 1.631644 & -2.646631 & -0.168840 \\ \mathrm{H} & -0.676916 & 2.370569 & 2.224488 \\ \mathrm{H} & -0.783836 & 0.667611 & 2.685956 \\ \mathrm{H} & -1.913981 & 3.244200 & 0.022986 \\ \mathrm{H} & -1.518446 & -1.741265 & 1.451010 \\ \mathrm{H} & -2.991264 & -3.368044 & 0.261809 \\ \mathrm{H} & -4.502380 & -2.611211 & -1.539980 \\ \mathrm{H} & 0.765510 & 2.726764 & 0.048899\end{array}$

Compound SI-PC-II

04

$\begin{array}{rrrr}\text { N } & 2.776823 & -2.902193 & -1.761432 \\ \mathrm{C} & 3.906859 & 0.961732 & 1.432361 \\ \mathrm{O} & 4.633907 & 0.451003 & 2.290953 \\ \mathrm{C} & 2.443526 & 0.527270 & 1.299373 \\ \mathrm{C} & 5.195672 & -3.602081 & -1.883743 \\ \mathrm{C} & 3.532782 & 2.648665 & -0.386444 \\ \mathrm{C} & 4.526529 & 2.916383 & -1.524176 \\ \mathrm{C} & 5.834046 & 3.192586 & -0.755828 \\ \mathrm{C} & 5.774178 & 2.258427 & 0.473776 \\ \mathrm{C} & 2.245204 & 1.943000 & -0.779774 \\ \mathrm{C} & 2.316617 & -1.020417 & 1.402508 \\ \mathrm{C} & 2.805609 & -1.800963 & 0.212847 \\ \mathrm{C} & 2.021531 & -2.199354 & -0.846039 \\ \mathrm{C} & 4.077690 & -2.982504 & -1.311833 \\ \mathrm{C} & 4.137548 & -2.293111 & -0.064876 \\ \mathrm{C} & 5.370878 & -2.238965 & 0.614233 \\ \mathrm{C} & 6.482798 & -2.854236 & 0.049027 \\ \mathrm{C} & 6.397844 & -3.529210 & -1.187804 \\ \mathrm{~N} & 1.792775 & 1.009941 & 0.085140 \\ \mathrm{~N} & 4.348365 & 1.907141 & 0.579551 \\ \mathrm{O} & 1.646077 & 2.272368 & -1.806272 \\ \mathrm{H} & 2.425542 & -3.302117 & -2.618368 \\ \mathrm{H} & 1.925285 & 0.942518 & 2.177500 \\ \mathrm{H} & 5.124965 & -4.121352 & -2.836012 \\ \mathrm{H} & 3.229981 & 3.612421 & 0.056255 \\ \mathrm{H} & 4.212553 & 3.746071 & -2.160699 \\ \mathrm{H} & 4.618321 & 2.019623 & -2.147218 \\ \mathrm{H} & 5.861786 & 4.238930 & -0.431940\end{array}$




\begin{tabular}{|c|c|c|c|}
\hline & & & \\
\hline & & & \\
\hline & & & \\
\hline & & & \\
\hline & & 989 & \\
\hline & 7210 & 808 & \\
\hline & חכם & 904 & \\
\hline & & 383 & \\
\hline & & & \\
\hline & & & \\
\hline & & 7543 & \\
\hline & & & \\
\hline & & & \\
\hline & & & \\
\hline & & & \\
\hline & & & \\
\hline & & & \\
\hline & & & \\
\hline & & & \\
\hline & & & \\
\hline & & & \\
\hline & & -2 & \\
\hline & & & \\
\hline & & & \\
\hline & & & \\
\hline & & & \\
\hline & & & \\
\hline & & & \\
\hline & & & \\
\hline & & & \\
\hline & & & \\
\hline & & & \\
\hline & & & \\
\hline & & & \\
\hline & -0.8 & & \\
\hline & & & \\
\hline & & & \\
\hline $\mathrm{H}$ & & & \\
\hline $\mathrm{H}$ & & & \\
\hline C & & & \\
\hline C & & & \\
\hline C & & & \\
\hline C & -1.9 & & \\
\hline $\mathrm{H}$ & -3.8 & -1 & \\
\hline r & -0.86 & & \\
\hline r & -3.3 & -4 & \\
\hline & & & \\
\hline O & & & \\
\hline$S$ & & & \\
\hline C & & & \\
\hline & & & \\
\hline & & & \\
\hline & 510 & & 1.5 \\
\hline
\end{tabular}

TS from SI-PC-II to SI-R-II

\begin{tabular}{|c|c|c|c|}
\hline & & & \\
\hline V & 2.868669 & -2.928062 & \\
\hline & & 1.017824 & \\
\hline & 894 & 8841 & \\
\hline & 243 & & \\
\hline & 5.306654 & -3.5 & -1 \\
\hline & 526 & 64 & \\
\hline 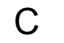 & 3.6 & & \\
\hline c & 4.9 & & \\
\hline C & 393 & & \\
\hline C & 1.7 & & \\
\hline c & 2.2 & -1.1 & \\
\hline$C$ & & & \\
\hline C & & & \\
\hline$C$ & & -2. & \\
\hline c & & & \\
\hline C & & & \\
\hline$C$ & & & \\
\hline$C$ & & & \\
\hline V & & & \\
\hline 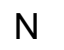 & & & \\
\hline 0 & & & \\
\hline & & & \\
\hline & & & \\
\hline & & & \\
\hline & & & \\
\hline & & & \\
\hline$H$ & & & \\
\hline $\mathrm{H}$ & & & \\
\hline$H$ & & & \\
\hline$H$ & & & \\
\hline H & & & \\
\hline$H$ & & & \\
\hline $\mathrm{H}$ & & & \\
\hline $\mathrm{H}$ & & & \\
\hline $\mathrm{H}$ & & & \\
\hline $\mathrm{H}$ & & & \\
\hline $\mathrm{H}$ & & & \\
\hline $\mathrm{H}$ & & & \\
\hline $\mathrm{Fe}$ & -2.2 & & \\
\hline $\mathrm{N}$ & -2.6 & & \\
\hline $\mathrm{N}$ & & & \\
\hline $\mathrm{N}$ & & & \\
\hline $\mathrm{N}$ & & & \\
\hline C & & & \\
\hline C & & & \\
\hline C & & & \\
\hline C & & & \\
\hline C & & & \\
\hline C & & & \\
\hline$\Omega$ & & & \\
\hline C & & & \\
\hline C & & & \\
\hline 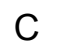 & & & \\
\hline & & 2.684785 & \\
\hline
\end{tabular}




$\begin{array}{lrrr}\text { C } & -0.680314 & 3.832687 & 1.181731 \\ \text { C } & -4.186768 & -3.076704 & -2.172127 \\ \text { C } & -1.323915 & -1.507698 & 3.939273 \\ \text { C } & -3.888165 & -3.779887 & -1.043658 \\ \text { C } & -1.817547 & -2.644832 & 3.372746 \\ \text { H } & -3.790244 & 1.470445 & -4.739083 \\ H & -0.109847 & 3.549496 & 3.294635 \\ \text { H } & -2.530500 & 3.646511 & -3.712583 \\ \text { H } & -0.454076 & 4.863908 & 0.944095 \\ \text { H } & -4.682330 & -3.419791 & -3.070894 \\ \text { H } & -0.922212 & -1.367003 & 4.934217 \\ \text { H } & -4.090240 & -4.819559 & -0.821373 \\ \text { H } & -1.909301 & -3.631864 & 3.806910 \\ \mathrm{C} & -3.795906 & -0.712533 & -2.904407 \\ \mathrm{C} & -0.966628 & 0.833425 & 3.123150 \\ \mathrm{C} & -2.796395 & -3.187541 & 1.134301 \\ \mathrm{C} & -1.615953 & 3.190579 & -1.050092 \\ \mathrm{H} & -4.277225 & -0.946674 & -3.848453 \\ \mathrm{H} & -0.569300 & 1.090650 & 4.099662 \\ \mathrm{H} & -2.936725 & -4.211640 & 1.464787 \\ \mathrm{H} & -1.398308 & 4.190028 & -1.409585 \\ \mathrm{O} & -0.708696 & -0.527651 & -0.580195 \\ \mathrm{~S} & -4.346376 & 0.809579 & 0.771725 \\ \mathrm{C} & -5.039156 & -0.304895 & 2.049446 \\ \mathrm{H} & -4.440599 & -0.293232 & 2.963552 \\ \mathrm{H} & -6.043096 & 0.064705 & 2.281322 \\ \mathrm{H} & -5.120011 & -1.330381 & 1.680053\end{array}$

Compound SI-R-II

$\begin{array}{lrrr}02 & & & \\ \text { N } & 2.644043 & -1.781181 & -1.140983 \\ \mathrm{C} & -1.130295 & 0.808909 & 0.799360 \\ \mathrm{O} & -0.283326 & 1.685774 & 0.916388 \\ \mathrm{C} & -0.917929 & -0.637102 & 1.384117 \\ \mathrm{C} & 4.381714 & 0.032908 & -1.371507 \\ \mathrm{C} & -3.322762 & -0.099010 & 0.076513 \\ \mathrm{C} & -4.134917 & 0.324925 & -1.157262 \\ \mathrm{C} & -4.111774 & 1.862295 & -1.064586 \\ \mathrm{C} & -2.709488 & 2.196369 & -0.519161 \\ \mathrm{C} & -2.678551 & -1.481357 & 0.004227 \\ \mathrm{C} & 0.543061 & -0.888083 & 1.793187 \\ \mathrm{C} & 1.519784 & -0.990193 & 0.652609 \\ \mathrm{C} & 1.598576 & -2.009132 & -0.272380 \\ \mathrm{C} & 3.276346 & -0.604311 & -0.796553 \\ \mathrm{C} & 2.590200 & -0.074818 & 0.333420 \\ \mathrm{C} & 3.043337 & 1.136104 & 0.889126 \\ \mathrm{C} & 4.143521 & 1.770833 & 0.324898 \\ \mathrm{C} & 4.807152 & 1.225156 & -0.794924 \\ \mathrm{~N} & -1.380538 & -1.611196 & 0.438871 \\ \mathrm{~N} & -2.316731 & 0.965127 & 0.187870 \\ \mathrm{O} & -3.303049 & -2.444474 & -0.439334 \\ \mathrm{H} & 2.896872 & -2.379485 & -1.913237 \\ \mathrm{H} & -1.556082 & -0.647103 & 2.288131 \\ \mathrm{H} & 4.889063 & -0.388969 & -2.235061 \\ \mathrm{H} & -3.971670 & -0.095328 & 0.967502\end{array}$

$\begin{array}{rrrr}\mathrm{H} & -5.142356 & -0.095347 & -1.147955 \\ \mathrm{H} & -3.629067 & -0.019938 & -2.066013 \\ \mathrm{H} & -4.878412 & 2.205780 & -0.361387 \\ \mathrm{H} & -4.300826 & 2.345534 & -2.026451 \\ \mathrm{H} & -2.706319 & 3.050561 & 0.164191 \\ \mathrm{H} & -1.984916 & 2.399219 & -1.315784 \\ \mathrm{H} & 0.547744 & -1.813649 & 2.381404 \\ \mathrm{H} & 0.843356 & -0.084002 & 2.470554 \\ \mathrm{H} & 0.978621 & -2.886849 & -0.379164 \\ \mathrm{H} & 2.527822 & 1.576402 & 1.737441 \\ \mathrm{H} & 4.498472 & 2.706920 & 0.748002 \\ \mathrm{H} & 5.663427 & 1.745647 & -1.215417\end{array}$

TS from SI-R-II to SI-R-III

02

$\begin{array}{rrrr}\text { N } & 2.054588 & -1.702855 & -0.920267 \\ \mathrm{C} & -1.285375 & 0.527119 & 1.430980 \\ \mathrm{O} & -0.896443 & 1.486723 & 2.105933 \\ \mathrm{C} & -0.832201 & -0.921629 & 1.700817 \\ \mathrm{C} & 3.141042 & 0.408001 & -1.773276 \\ \mathrm{C} & -2.587282 & -0.472826 & -0.440516 \\ \mathrm{C} & -3.056809 & 0.224715 & -1.723386 \\ \mathrm{C} & -3.609956 & 1.566608 & -1.209244 \\ \mathrm{C} & -2.660827 & 1.949133 & -0.060258 \\ \mathrm{C} & -1.436582 & -1.483161 & -0.612862 \\ \mathrm{C} & 0.584753 & -0.934887 & 2.333706 \\ \mathrm{C} & 1.508491 & -0.972254 & 1.156546 \\ \mathrm{C} & 1.423875 & -2.059332 & 0.237002 \\ \mathrm{C} & 2.496783 & -0.389081 & -0.832173 \\ \mathrm{C} & 2.161418 & 0.089889 & 0.471328 \\ \mathrm{C} & 2.474986 & 1.422340 & 0.814730 \\ \mathrm{C} & 3.126193 & 2.216795 & -0.118700 \\ \mathrm{C} & 3.457796 & 1.714810 & -1.394976 \\ \mathrm{~N} & -0.745542 & -1.775335 & 0.508010 \\ \mathrm{~N} & -2.155522 & 0.651531 & 0.409468 \\ \mathrm{O} & -1.227853 & -2.011393 & -1.713728 \\ \mathrm{H} & 2.124856 & -2.289303 & -1.739226 \\ \mathrm{H} & -1.560154 & -1.353338 & 2.407149 \\ \mathrm{H} & 3.384922 & 0.033421 & -2.762814 \\ \mathrm{H} & -3.431780 & -0.996424 & 0.035195 \\ \mathrm{H} & -3.796192 & -0.368235 & -2.266828 \\ \mathrm{H} & -2.197804 & 0.378683 & -2.384721 \\ \mathrm{H} & -4.627634 & 1.432125 & -0.825003 \\ \mathrm{H} & -3.645947 & 2.336580 & -1.984868 \\ \mathrm{H} & -3.157964 & 2.485818 & 0.753694 \\ \mathrm{H} & -1.821869 & 2.567573 & -0.402367 \\ \mathrm{H} & 0.706991 & -1.828549 & 2.955336 \\ \mathrm{H} & 0.723044 & -0.051847 & 2.958568 \\ \mathrm{H} & 1.233769 & -3.099462 & 0.444966 \\ \mathrm{H} & 2.202206 & 1.815093 & 1.789175 \\ \mathrm{H} & 3.380256 & 3.242796 & 0.130272 \\ \mathrm{H} & 3.965163 & 2.361605 & -2.104951\end{array}$

Compound SI-R-III

02 


$\begin{array}{rrrr}\text { N } & -1.634781 & -1.658743 & -0.712899 \\ \mathrm{C} & 1.506274 & 1.567482 & -0.154578 \\ \mathrm{O} & 1.289192 & 2.670708 & 0.346353 \\ \mathrm{C} & 0.755073 & 1.038723 & -1.387324 \\ \mathrm{C} & -3.304927 & -1.425570 & 1.142572 \\ \mathrm{C} & 2.677383 & -0.633732 & -0.275697 \\ \mathrm{C} & 3.411967 & -1.374954 & 0.849997 \\ \mathrm{C} & 4.199742 & -0.253039 & 1.549491 \\ \mathrm{C} & 3.250679 & 0.956017 & 1.502868 \\ \mathrm{C} & 1.368101 & -1.288041 & -0.726507 \\ \mathrm{C} & -0.635159 & 1.703001 & -1.624591 \\ \mathrm{C} & -1.597803 & 0.590322 & -1.339798 \\ \mathrm{C} & -0.930233 & -0.743561 & -1.583446 \\ \mathrm{C} & -2.516513 & -0.960855 & 0.094344 \\ \mathrm{C} & -2.538967 & 0.420425 & -0.299025 \\ \mathrm{C} & -3.374652 & 1.321822 & 0.393154 \\ \mathrm{C} & -4.168361 & 0.849252 & 1.434670 \\ \mathrm{C} & -4.133360 & -0.507518 & 1.803256 \\ \mathrm{~N} & 0.474856 & -0.406728 & -1.227093 \\ \mathrm{~N} & 2.439614 & 0.703610 & 0.301756 \\ \mathrm{O} & 1.162150 & -2.500586 & -0.626376 \\ \mathrm{H} & -1.174033 & -2.512178 & -0.426085 \\ \mathrm{H} & 1.408610 & 1.193394 & -2.257627 \\ \mathrm{H} & -3.272493 & -2.467841 & 1.446986 \\ \mathrm{H} & 3.329629 & -0.547663 & -1.159799 \\ \mathrm{H} & 4.046275 & -2.176432 & 0.465264 \\ \mathrm{H} & 2.679024 & -1.822222 & 1.529485 \\ \mathrm{H} & 5.116531 & -0.031114 & 0.991515 \\ \mathrm{H} & 4.485357 & -0.511679 & 2.572487 \\ \mathrm{H} & 3.769873 & 1.914922 & 1.417145 \\ \mathrm{H} & 2.598707 & 1.005172 & 2.383523 \\ \mathrm{H} & -0.696716 & 2.068473 & -2.657054 \\ \mathrm{H} & -0.758934 & 2.559516 & -0.957255 \\ \mathrm{H} & -0.943585 & -1.108738 & -2.620713 \\ \mathrm{H} & -3.394087 & 2.371118 & 0.111483 \\ \mathrm{H} & -4.819868 & 1.533436 & 1.970662 \\ \mathrm{H} & -4.758319 & -0.854737 & 2.621519\end{array}$

TS from SI-R-III to SI-R-IV 02
N 0.265708
$0.489845-1.431330$
C -3.102061
$-0.299133$
1.399508
O -3.569103
0.553118
2.155126
C -1.626946
$-0.733673$
1.418794
C -0.303932
2.861026
$-2.044520$
C -3.256682
$-1.963198$
$-0.448991$
C -4.309087
$-2.047212$
$-1.563253$
C -5.628170
$-1.769166$
$-0.820289$
C -5.252241
$-0.703634$
0.222744
C -1.859650
$-1.542618$
$-0.916885$
C -0.658815
0.290992
2.059553
C 0.237476
0.763580
0.922416
C 0.185109
$-0.276263$
$-0.201179$
C -0.049512
1.820228
$-1.152825$
C $\quad-0.055024$
2.034553
0.246374

$\begin{array}{rrrr}\mathrm{C} & -0.303496 & 3.312377 & 0.751533 \\ \mathrm{C} & -0.553996 & 4.364950 & -0.137298 \\ \mathrm{C} & -0.554612 & 4.135698 & -1.518381 \\ \mathrm{~N} & -1.135600 & -0.922788 & 0.040417 \\ \mathrm{~N} & -3.829957 & -0.971780 & 0.481196 \\ \mathrm{O} & -1.458994 & -1.748919 & -2.066965 \\ \mathrm{H} & -0.111076 & 0.036184 & -2.256286 \\ \mathrm{H} & -1.583391 & -1.690948 & 1.959163 \\ \mathrm{H} & -0.314333 & 2.688517 & -3.117350 \\ \mathrm{H} & -3.161243 & -2.938052 & 0.056518 \\ \mathrm{H} & -4.292303 & -3.014701 & -2.069735 \\ \mathrm{H} & -4.108421 & -1.272667 & -2.310731 \\ \mathrm{H} & -5.976438 & -2.678506 & -0.317294 \\ \mathrm{H} & -6.425347 & -1.429515 & -1.486781 \\ \mathrm{H} & -5.828344 & -0.778003 & 1.149628 \\ \mathrm{H} & -5.371031 & 0.315547 & -0.165134 \\ \mathrm{H} & -0.094538 & -0.195297 & 2.859205 \\ \mathrm{H} & -1.231016 & 1.109055 & 2.503659 \\ \mathrm{H} & 0.967053 & -1.041636 & -0.163663 \\ \mathrm{H} & -0.316232 & 3.486458 & 1.825023 \\ \mathrm{H} & -0.753941 & 5.361395 & 0.246319 \\ \mathrm{H} & -0.756964 & 4.958333 & -2.199461 \\ \mathrm{~N} & 4.612971 & 0.341156 & -1.222571 \\ \mathrm{C} & 3.001693 & 1.230941 & 0.487554 \\ \mathrm{C} & 5.364913 & -3.165980 & -0.245745 \\ \mathrm{C} & 4.885589 & -1.753666 & -0.409610 \\ \mathrm{C} & 5.248712 & -0.871348 & -1.406146 \\ \mathrm{C} & 3.807945 & 0.267939 & -0.101945 \\ \mathrm{C} & 3.969451 & -1.046907 & 0.435380 \\ \mathrm{C} & 3.288990 & -1.369186 & 1.637875 \\ \mathrm{C} & 2.470190 & -0.435282 & 2.228870 \\ \mathrm{C} & 2.195451 & 0.850824 & 1.614223 \\ \mathrm{H} & 4.686095 & 1.135361 & -1.840357 \\ \mathrm{H} & 2.917798 & 2.229792 & 0.070476 \\ \mathrm{H} & 4.533512 & -3.882529 & -0.266057 \\ \mathrm{H} & 6.059179 & -3.441529 & -1.046287 \\ \mathrm{H} & 5.921990 & -1.016140 & -2.240212 \\ \mathrm{H} & 3.444542 & -2.336684 & 2.109677 \\ \mathrm{H} & 1.995766 & -0.670620 & 3.177130 \\ \mathrm{H} & 1.897289 & 1.653397 & 2.287298 \\ \mathrm{H} & 5.886538 & -3.310882 & 0.709442 \\ & & & \end{array}$

\section{Compound SI-R-IV}

02

$\begin{array}{lrrr}N & 0.098901 & 1.051300 & -1.574953 \\ C & -3.065573 & -0.584440 & 1.445621 \\ O & -3.536363 & -0.054982 & 2.452857 \\ C & -1.558598 & -0.638766 & 1.176721 \\ C & -0.102977 & 3.550690 & -1.712833 \\ C & -3.278238 & -1.784768 & -0.736549 \\ C & -4.466217 & -1.720217 & -1.707018 \\ C & -5.677065 & -1.903360 & -0.774946 \\ C & -5.288394 & -1.140623 & 0.504459 \\ C & -2.005637 & -1.099597 & -1.230571 \\ C & -0.767619 & 0.515645 & 1.809384\end{array}$




$\begin{array}{rrrr}\mathrm{C} & 0.387181 & 0.857149 & 0.812919 \\ \mathrm{C} & 0.063664 & 0.071998 & -0.505960 \\ \mathrm{C} & 0.068491 & 2.337354 & -1.041991 \\ \mathrm{C} & 0.303493 & 2.303019 & 0.343569 \\ \mathrm{C} & 0.386173 & 3.484933 & 1.068556 \\ \mathrm{C} & 0.226188 & 4.711746 & 0.406382 \\ \mathrm{C} & -0.020450 & 4.734052 & -0.969459 \\ \mathrm{~N} & -1.268575 & -0.515932 & -0.258831 \\ \mathrm{~N} & -3.816917 & -1.169769 & 0.487644 \\ \mathrm{O} & -1.689917 & -1.100503 & -2.424007 \\ \mathrm{H} & -0.416806 & 0.815927 & -2.414236 \\ \mathrm{H} & -1.184677 & -1.605931 & 1.548713 \\ \mathrm{H} & -0.293906 & 3.575357 & -2.782212 \\ \mathrm{H} & -3.016797 & -2.837888 & -0.540819 \\ \mathrm{H} & -4.398846 & -2.480233 & -2.488047 \\ \mathrm{H} & -4.489185 & -0.737588 & -2.190666 \\ \mathrm{H} & -5.819775 & -2.966234 & -0.549416 \\ \mathrm{H} & -6.606690 & -1.527118 & -1.209823 \\ \mathrm{H} & -5.671148 & -1.604733 & 1.418309 \\ \mathrm{H} & -5.631258 & -0.099353 & 0.489796 \\ \mathrm{H} & -0.401506 & 0.248634 & 2.803109 \\ \mathrm{H} & -1.427869 & 1.376228 & 1.933279 \\ \mathrm{H} & 0.770914 & -0.739261 & -0.706337 \\ \mathrm{H} & 0.561846 & 3.463945 & 2.141964 \\ \mathrm{H} & 0.287716 & 5.641520 & 0.964383 \\ \mathrm{H} & -0.152437 & 5.686520 & -1.476646 \\ \mathrm{~N} & 4.827437 & -0.008359 & -0.944487 \\ \mathrm{C} & 2.918302 & 0.852849 & 0.423305 \\ \mathrm{C} & 5.141064 & -3.633303 & -0.185239 \\ \mathrm{C} & 4.788338 & -2.179008 & -0.302936 \\ \mathrm{C} & 5.424120 & -1.244193 & -1.105212 \\ \mathrm{C} & 3.781910 & -0.114483 & -0.040617 \\ \mathrm{C} & 3.749720 & -1.484223 & 0.376989 \\ \mathrm{C} & 2.773378 & -1.867126 & 1.354234 \\ \mathrm{C} & 1.887564 & -0.960743 & 1.840349 \\ \mathrm{C} & 1.818897 & 0.484585 & 1.389363 \\ \mathrm{H} & 5.088543 & 0.832082 & -1.437509 \\ \mathrm{H} & 2.986814 & 1.882100 & 0.085806 \\ \mathrm{H} & 4.306991 & -4.280063 & -0.486891 \\ \mathrm{H} & 5.998766 & -3.882851 & -0.818355 \\ \mathrm{H} & 6.260925 & -1.369627 & -1.778436 \\ \mathrm{H} & 2.758423 & -2.890319 & 1.724470 \\ \mathrm{H} & 1.185055 & -1.272397 & 2.608673 \\ \mathrm{H} & 1.903544 & 1.126221 & 2.287369 \\ \mathrm{H} & 5.399407 & -3.906765 & 0.846009\end{array}$

Compound SI-PDT ${ }_{\text {C-C }}$

01

$\begin{array}{lrrr}N & -0.438727 & 0.477825 & -1.938170 \\ C & -2.844336 & -0.568978 & 1.584112 \\ O & -2.782404 & 0.067747 & 2.635303 \\ C & -1.645382 & -1.304830 & 0.960532 \\ C & -0.928155 & 2.910736 & -1.510061 \\ C & -4.022475 & -1.469358 & -0.415412 \\ C & -5.326405 & -0.976539 & -1.058309\end{array}$

\begin{tabular}{|c|c|c|c|}
\hline & & & \\
\hline & 692 & & \\
\hline & -2.761785 & & \\
\hline & -0.263357 & & \\
\hline & 1925 & 49 & \\
\hline & -0.331410 & -0.655094 & -1 \\
\hline & -0.494395 & 187 & \\
\hline & -0.004895 & & \\
\hline & 025 & & \\
\hline & -0.380719 & & \\
\hline & -0.862095 & & -0.5 \\
\hline & -1.637367 & -1.1 & \\
\hline & -3.974078 & -0.728873 & \\
\hline & -2.792362 & & \\
\hline & $-1.1<$ & & -2.6 \\
\hline & & & \\
\hline & & & \\
\hline & & & \\
\hline & & -1.7 & \\
\hline & & & \\
\hline & & $-1 . t$ & \\
\hline & & & \\
\hline & & & \\
\hline & & & \\
\hline & & -1 & \\
\hline & & -0 . & \\
\hline & & -1 & \\
\hline & & & \\
\hline & -0 . & & \\
\hline & -1. & & \\
\hline & & & \\
\hline & & & \\
\hline & & -1 . & \\
\hline & & & \\
\hline & & 0.5 & \\
\hline & & & \\
\hline & & $-0 . c$ & \\
\hline & & & \\
\hline & & -1.6 & \\
\hline & & -0.3 & \\
\hline & & 1.75 & \\
\hline & 6.92 & -2.58 & -0.5 \\
\hline & 8.129501 & -1.623 & -0 \\
\hline & 7.410997 & 0.990461 & 0.0 \\
\hline & 4.093022 & -2.991383 & -0.0 \\
\hline & 1.696742 & -2.490085 & \\
\hline & 6.9 & -2.653529 & \\
\hline & 5.253870 & 2.274163 & 0.153 \\
\hline
\end{tabular}

Compound SI-C-III

11

$\begin{array}{rrrr}\text { N } & -1.653726 & -1.554632 & -0.457652 \\ \mathrm{C} & 1.744958 & 1.562715 & -0.100231 \\ \mathrm{O} & 1.638618 & 2.676386 & 0.408882 \\ \mathrm{C} & 0.802434 & 1.074953 & -1.212377\end{array}$ 


$\begin{array}{rrrr}\mathrm{C} & -3.770019 & -1.468938 & 0.842437 \\ \mathrm{C} & 2.770709 & -0.708030 & -0.344519 \\ \mathrm{C} & 3.588057 & -1.483869 & 0.699652 \\ \mathrm{C} & 4.519506 & -0.404333 & 1.277999 \\ \mathrm{C} & 3.645765 & 0.859707 & 1.328363 \\ \mathrm{C} & 1.394802 & -1.301192 & -0.645228 \\ \mathrm{C} & -0.572421 & 1.806822 & -1.245228 \\ \mathrm{C} & -1.536262 & 0.702769 & -0.979658 \\ \mathrm{C} & -0.911778 & -0.627608 & -1.292280 \\ \mathrm{C} & -2.720259 & -0.934950 & 0.063988 \\ \mathrm{C} & -2.655577 & 0.500486 & -0.219783 \\ \mathrm{C} & -3.621385 & 1.386893 & 0.361522 \\ \mathrm{C} & -4.615169 & 0.844769 & 1.123256 \\ \mathrm{C} & -4.683339 & -0.574383 & 1.351158 \\ \mathrm{~N} & 0.473922 & -0.352342 & -0.976457 \\ \mathrm{~N} & 2.674684 & 0.646096 & 0.239742 \\ \mathrm{O} & 1.132005 & -2.496908 & -0.591291 \\ \mathrm{H} & -1.505145 & -2.556142 & -0.493200 \\ \mathrm{H} & 1.316747 & 1.184702 & -2.175732 \\ \mathrm{H} & -3.837361 & -2.532144 & 1.042767 \\ \mathrm{H} & 3.320315 & -0.665772 & -1.298334 \\ \mathrm{H} & 4.122081 & -2.325160 & 0.253641 \\ \mathrm{H} & 2.917386 & -1.877840 & 1.470523 \\ \mathrm{H} & 5.371984 & -0.244943 & 0.608806 \\ \mathrm{H} & 4.910962 & -0.670994 & 2.262627 \\ \mathrm{H} & 4.205211 & 1.783673 & 1.159792 \\ \mathrm{H} & 3.109848 & 0.956902 & 2.279703 \\ \mathrm{H} & -0.748162 & 2.278716 & -2.218935 \\ \mathrm{H} & -0.609577 & 2.590263 & -0.483680 \\ \mathrm{H} & -1.050071 & -0.923941 & -2.345653 \\ \mathrm{H} & -3.562730 & 2.452737 & 0.170325 \\ \mathrm{H} & -5.375267 & 1.477656 & 1.567687 \\ \mathrm{H} & -5.495508 & -0.952256 & 1.965084\end{array}$

TS from SI-C-III to SI-C-IV 11
$N$
C $\quad-3.035039$
$0.644691-1.446866$
O -3.531108
$-0.389201$
1.421128
C -1.531379
0.407198
2.215769
C -0.269550
$-0.715268$
1.398055
C -3.112645
$3.011137-2.043863$
C -4.183456
$\begin{array}{ll}-1.999073 & -0.483448\end{array}$
C -5.499021
$-2.123295$
$-1.577146$
C -5.175577
$-1.963915$
$-0.793860$
$-1.759754$
$-0.904735$
0.271996
C -0.636728
$-1.474374$
$-0.968661$
C $\quad 0.190495$
0.371172
2.049927
C $\quad 0.220127$
0.889011
0.886202
$\begin{array}{ll}\text { C } & -0.018186 \\ \text { C } & -0.057927\end{array}$
$-0.147416$
$-0.237137$
1.949740
$-1.162944$
C -0.353364
2.147597
0.250339
C -0.596875
3.418641
0.782611
C -0.552690
4.464374
$-0.090186$
N $\quad-1.066862$
4.252805
$-1.489209$
$-0.816354$
$-0.001759$

$\begin{array}{rrrr}\mathrm{N} & -3.729250 & -1.076520 & 0.490697 \\ \mathrm{O} & -1.346541 & -1.626115 & -2.116799 \\ \mathrm{H} & 0.063298 & 0.197034 & -2.328912 \\ \mathrm{H} & -1.390797 & -1.677693 & 1.908673 \\ \mathrm{H} & -0.249759 & 2.864305 & -3.118668 \\ \mathrm{H} & -2.936877 & -2.979302 & -0.011989 \\ \mathrm{H} & -4.109473 & -3.071746 & -2.112988 \\ \mathrm{H} & -4.058485 & -1.313680 & -2.303678 \\ \mathrm{H} & -5.768343 & -2.910457 & -0.312289 \\ \mathrm{H} & -6.333961 & -1.662946 & -1.431453 \\ \mathrm{H} & -5.719284 & -1.047853 & 1.209713 \\ \mathrm{H} & -5.373795 & 0.114107 & -0.081442 \\ \mathrm{H} & -0.037598 & -0.066421 & 2.846452 \\ \mathrm{H} & -1.260423 & 1.155482 & 2.483610 \\ \mathrm{H} & 1.042700 & -0.869174 & -0.197649 \\ \mathrm{H} & -0.386353 & 3.569928 & 1.857523 \\ \mathrm{H} & -0.827775 & 5.452743 & 0.292730 \\ \mathrm{H} & -0.754449 & 5.089075 & -2.152370 \\ \mathrm{~N} & 4.420825 & 0.137988 & -1.342505 \\ \mathrm{C} & 2.951644 & 1.204971 & 0.387142 \\ \mathrm{C} & 5.123178 & -3.304831 & -0.128381 \\ \mathrm{C} & 4.678623 & -1.892112 & -0.363693 \\ \mathrm{C} & 4.999878 & -1.093182 & -1.454821 \\ \mathrm{C} & 3.701266 & 0.187841 & -0.163676 \\ \mathrm{C} & 3.855940 & -1.090782 & 0.472363 \\ \mathrm{C} & 3.234678 & -1.307586 & 1.730636 \\ \mathrm{C} & 2.494841 & -0.296450 & 2.296499 \\ \mathrm{C} & 2.275395 & 0.950977 & 1.616826 \\ \mathrm{H} & 4.509435 & 0.887728 & -2.014137 \\ \mathrm{H} & 2.853375 & 2.168753 & -0.102255 \\ \mathrm{H} & 4.268135 & -3.987492 & -0.054004 \\ \mathrm{H} & 5.760536 & -3.656043 & -0.944905 \\ \mathrm{H} & 5.616076 & -1.332193 & -2.311271 \\ \mathrm{H} & 3.370866 & -2.250598 & 2.251348 \\ \mathrm{H} & 2.063810 & -0.436985 & 3.282400 \\ \mathrm{H} & 1.971117 & 1.806099 & 2.212811 \\ \mathrm{H} & 5.693454 & -3.396254 & 0.803823 \\ & & & \end{array}$

\section{Compound SI-C-IV}

11

$\begin{array}{lrrr}\text { N } & 0.168642 & 0.394571 & -1.466474 \\ \text { C } & -3.072888 & -0.431485 & 1.424746 \\ \text { O } & -3.381382 & 0.357176 & 2.316885 \\ \text { C } & -1.668132 & -1.048162 & 1.294805 \\ \text { C } & -0.641526 & 2.772177 & -1.691039 \\ \text { C } & -3.515386 & -1.785976 & -0.618621 \\ \text { C } & -4.618686 & -1.589365 & -1.668184 \\ \text { C } & -5.857771 & -1.263766 & -0.815443 \\ \text { C } & -5.306373 & -0.403603 & 0.333660 \\ \text { C } & -2.101522 & -1.468799 & -1.111697 \\ \text { C } & -0.555606 & -0.271073 & 2.021804 \\ \text { C } & 0.352193 & 0.311056 & 0.910478 \\ C & 0.094140 & -0.550155 & -0.364885 \\ \text { C } & -0.231841 & 1.646064 & -0.976878 \\ \text { C } & -0.112376 & 1.676774 & 0.422680\end{array}$




$\begin{array}{rrrr}\mathrm{C} & -0.388286 & 2.842772 & 1.128523 \\ \mathrm{C} & -0.798885 & 3.981136 & 0.423350 \\ \mathrm{C} & -0.923516 & 3.937355 & -0.969907 \\ \mathrm{~N} & -1.259926 & -1.080984 & -0.119984 \\ \mathrm{~N} & -3.917616 & -0.872197 & 0.469045 \\ \mathrm{O} & -1.773660 & -1.547210 & -2.296960 \\ \mathrm{H} & -0.245371 & 0.055398 & -2.330422 \\ \mathrm{H} & -1.730707 & -2.073238 & 1.686633 \\ \mathrm{H} & -0.742231 & 2.744607 & -2.772042 \\ \mathrm{H} & -3.518144 & -2.826036 & -0.254411 \\ \mathrm{H} & -4.745298 & -2.472641 & -2.297613 \\ \mathrm{H} & -4.356684 & -0.747306 & -2.317050 \\ \mathrm{H} & -6.294573 & -2.186115 & -0.416554 \\ \mathrm{H} & -6.634128 & -0.743444 & -1.382000 \\ \mathrm{H} & -5.844700 & -0.536664 & 1.276164 \\ \mathrm{H} & -5.311186 & 0.665698 & 0.090407 \\ \mathrm{H} & -0.017079 & -0.941926 & 2.691280 \\ \mathrm{H} & -0.997211 & 0.517358 & 2.634675 \\ \mathrm{H} & 0.800398 & -1.372166 & -0.512287 \\ \mathrm{H} & -0.302018 & 2.873679 & 2.211940 \\ \mathrm{H} & -1.028041 & 4.896268 & 0.960380 \\ \mathrm{H} & -1.250461 & 4.823887 & -1.506250 \\ \mathrm{~N} & 4.779457 & 0.844622 & -1.151920 \\ \mathrm{C} & 2.714170 & 0.992737 & 0.227382 \\ \mathrm{C} & 6.429390 & -2.348788 & -0.210321 \\ \mathrm{C} & 5.543922 & -1.148574 & -0.366203 \\ \mathrm{C} & 5.758958 & -0.070808 & -1.249783 \\ \mathrm{C} & 3.862599 & 0.415356 & -0.193826 \\ \mathrm{C} & 4.352643 & -0.851884 & 0.303651 \\ \mathrm{C} & 3.599154 & -1.529113 & 1.315608 \\ \mathrm{C} & 2.451074 & -0.974347 & 1.775609 \\ \mathrm{C} & 1.902901 & 0.336331 & 1.294319 \\ \mathrm{H} & 4.719801 & 1.701220 & -1.687705 \\ \mathrm{H} & 2.371589 & 1.940826 & -0.173234 \\ \mathrm{H} & 5.915727 & -3.264608 & -0.524454 \\ \mathrm{H} & 7.334612 & -2.248246 & -0.814988 \\ \mathrm{H} & 6.578373 & 0.069944 & -1.943049 \\ \mathrm{H} & 3.956770 & -2.473638 & 1.712877 \\ \mathrm{H} & 1.905312 & -1.482826 & 2.561932 \\ \mathrm{H} & 1.937171 & 1.026408 & 2.159582 \\ \mathrm{H} & 6.734403 & -2.483403 & 0.833155\end{array}$

Figure S23B Radical C2 TS 02

$\begin{array}{lrrr}\text { N } & 2.714778 & -0.758670 & 1.803598 \\ \text { C } & 3.944439 & -2.701281 & 0.772631 \\ \text { C } & 3.440973 & 2.172721 & -0.375352 \\ \text { C } & 3.276231 & 0.843215 & 0.290893 \\ \text { C } & 2.468096 & 0.569027 & 1.427761 \\ \text { C } & 3.507543 & -1.378260 & 0.861669 \\ \text { C } & 3.875989 & -0.388977 & -0.102480 \\ \text { C } & 4.717248 & -0.762373 & -1.171968 \\ \text { C } & 5.159781 & -2.077524 & -1.256967 \\ \text { C } & 4.776917 & -3.036769 & -0.296497 \\ \text { H } & 2.315984 & -1.212909 & 2.610401\end{array}$

$\begin{array}{rrrr}\mathrm{H} & 3.652981 & -3.442190 & 1.512097 \\ \mathrm{H} & 2.896273 & 2.223553 & -1.327301 \\ \mathrm{H} & 3.068620 & 2.984899 & 0.255797 \\ \mathrm{H} & 2.249127 & 1.288281 & 2.208969 \\ \mathrm{H} & 5.012467 & -0.030086 & -1.918835 \\ \mathrm{H} & 5.808033 & -2.375661 & -2.076517 \\ \mathrm{H} & 5.137357 & -4.057695 & -0.388453 \\ \mathrm{H} & 4.497292 & 2.372197 & -0.597641 \\ \mathrm{~N} & 0.237898 & 0.592625 & -1.530339 \\ \mathrm{C} & -2.907903 & -0.400917 & 1.474694 \\ \mathrm{O} & -3.284239 & 0.382243 & 2.346842 \\ \mathrm{C} & -1.451716 & -0.875370 & 1.333131 \\ \mathrm{C} & -0.345976 & 3.014071 & -1.872188 \\ \mathrm{C} & -3.263977 & -1.865202 & -0.510805 \\ \mathrm{C} & -4.400168 & -1.801877 & -1.540997 \\ \mathrm{C} & -5.648013 & -1.567559 & -0.671164 \\ \mathrm{C} & -5.156194 & -0.628298 & 0.442965 \\ \mathrm{C} & -1.891573 & -1.441454 & -1.044418 \\ \mathrm{C} & -0.402941 & 0.059412 & 1.987616 \\ \mathrm{C} & 0.384180 & 0.643635 & 0.827352 \\ \mathrm{C} & 0.236410 & -0.285853 & -0.376624 \\ \mathrm{C} & -0.035763 & 1.893012 & -1.105712 \\ \mathrm{C} & 0.073350 & 1.972228 & 0.306369 \\ \mathrm{C} & -0.120897 & 3.199507 & 0.947371 \\ \mathrm{C} & -0.423074 & 4.332120 & 0.182392 \\ \mathrm{C} & -0.534593 & 4.235436 & -1.210224 \\ \mathrm{~N} & -1.073428 & -0.942483 & -0.091731 \\ \mathrm{~N} & -3.727211 & -0.956552 & 0.555517 \\ \mathrm{O} & -1.588171 & -1.541287 & -2.237306 \\ \mathrm{H} & -0.199164 & 0.223267 & -2.367341 \\ \mathrm{H} & -1.399270 & -1.880670 & 1.776458 \\ \mathrm{H} & -0.444551 & 2.943542 & -2.952049 \\ \mathrm{H} & -3.169362 & -2.888904 & -0.113658 \\ \mathrm{H} & -4.456364 & -2.710294 & -2.144656 \\ \mathrm{H} & -4.229093 & -0.958139 & -2.217519 \\ \mathrm{H} & -5.989578 & -2.514767 & -0.238440 \\ \mathrm{H} & -6.480858 & -1.137123 & -1.233309 \\ \mathrm{H} & -5.660140 & -0.786924 & 1.400823 \\ \mathrm{H} & -5.271793 & 0.428904 & 0.174379 \\ \mathrm{H} & 0.220693 & -0.525185 & 2.672244 \\ \mathrm{H} & -0.909953 & 0.826679 & 2.577619 \\ \mathrm{H} & 1.003690 & -1.060712 & -0.475237 \\ \mathrm{H} & -0.051309 & 3.271986 & 2.030270 \\ \mathrm{H} & -0.578874 & 5.289279 & 0.671972 \\ & -0.777407 & 5.120568 & -1.792375\end{array}$

Figure S23B Radical C2 Product 02

$\begin{array}{lrrr}\text { N } & 1.989245 & -1.239008 & 1.313234 \\ \text { C } & 3.473386 & -3.169335 & 0.716716 \\ \text { C } & 3.540872 & 1.671307 & -0.564656 \\ \text { C } & 3.104180 & 0.371460 & 0.027350 \\ \text { C } & 2.010592 & 0.216728 & 1.068173 \\ \text { C } & 3.051171 & -1.848357 & 0.654952 \\ \text { C } & 3.701829 & -0.886102 & -0.182984\end{array}$




$\begin{array}{rrrr}\mathrm{C} & 4.780775 & -1.309937 & -0.996541 \\ \mathrm{C} & 5.196149 & -2.637831 & -0.938317 \\ \mathrm{C} & 4.557580 & -3.557643 & -0.089115 \\ \mathrm{H} & 1.767606 & -1.556833 & 2.248179 \\ \mathrm{H} & 2.978469 & -3.884855 & 1.367961 \\ \mathrm{H} & 2.857418 & 2.025325 & -1.348168 \\ \mathrm{H} & 3.585354 & 2.465202 & 0.191765 \\ \mathrm{H} & 2.289873 & 0.757330 & 1.991105 \\ \mathrm{H} & 5.285297 & -0.603088 & -1.649460 \\ \mathrm{H} & 6.027721 & -2.967990 & -1.555100 \\ \mathrm{H} & 4.902858 & -4.587232 & -0.054931 \\ \mathrm{H} & 4.535298 & 1.576800 & -1.014210 \\ \mathrm{~N} & 0.054983 & 1.282895 & -1.599473 \\ \mathrm{C} & -2.825086 & -0.758997 & 1.480920 \\ \mathrm{O} & -3.163962 & -0.402773 & 2.610191 \\ \mathrm{C} & -1.360601 & -0.775411 & 1.035390 \\ \mathrm{C} & -0.075611 & 3.778144 & -1.343442 \\ \mathrm{C} & -3.319260 & -1.578969 & -0.829262 \\ \mathrm{C} & -4.605521 & -1.332591 & -1.630673 \\ \mathrm{C} & -5.709313 & -1.657777 & -0.608692 \\ \mathrm{C} & -5.154541 & -1.133735 & 0.728457 \\ \mathrm{C} & -2.085615 & -0.844045 & -1.348495 \\ \mathrm{C} & -0.482044 & 0.268094 & 1.739982 \\ \mathrm{C} & 0.580212 & 0.729453 & 0.687629 \\ \mathrm{C} & 0.091414 & 0.154869 & -0.687664 \\ \mathrm{C} & 0.123273 & 2.475322 & -0.881381 \\ \mathrm{C} & 0.510267 & 2.230003 & 0.447543 \\ \mathrm{C} & 0.712971 & 3.287724 & 1.324982 \\ \mathrm{C} & 0.529376 & 4.602623 & 0.870839 \\ \mathrm{C} & 0.134092 & 4.835037 & -0.449748 \\ \mathrm{~N} & -1.229104 & -0.431690 & -0.386474 \\ \mathrm{~N} & -3.695847 & -1.169960 & 0.533679 \\ \mathrm{O} & -1.899690 & -0.662650 & -2.555131 \\ \mathrm{H} & -0.557035 & 1.185508 & -2.400915 \\ \mathrm{H} & -0.961399 & -1.786217 & 1.210227 \\ \mathrm{H} & -0.381662 & 3.966355 & -2.368775 \\ \mathrm{H} & -3.076206 & -2.654331 & -0.842107 \\ \mathrm{H} & -4.651417 & -1.947840 & -2.531490 \\ \mathrm{H} & -4.650210 & -0.281142 & -1.934836 \\ \mathrm{H} & -5.861322 & -2.741590 & -0.553016 \\ \mathrm{H} & -6.668689 & -1.199157 & -0.861746 \\ \mathrm{H} & -5.442932 & -1.748148 & 1.586592 \\ \mathrm{H} & -5.467528 & -0.103657 & 0.935504 \\ \mathrm{H} & -0.030474 & -0.141514 & 2.646563 \\ & -1.101879 & 1.113139 & 2.046043 \\ \mathrm{H} & 1.000551 & -0.634175 & -1.079566 \\ \mathrm{H} & 5.103364 & 2.357720 \\ \mathrm{H} & 5.855066 & -0.794077\end{array}$

Figure S23B Radical C3 TS

02

$\begin{array}{lrrr}\mathrm{N} & 0.447129 & -0.050752 & -1.463984 \\ \mathrm{C} & -2.919196 & 0.064156 & 1.391562 \\ \mathrm{O} & -3.069446 & 0.981014 & 2.199269\end{array}$

\begin{tabular}{|c|c|c|c|}
\hline & -1.6 & & \\
\hline & 69 & 325 & \\
\hline & & & \\
\hline & -4.653126 & 27837 & \\
\hline & -5.798378 & -0.360273 & \\
\hline & -5.080094 & 1348 & 317 \\
\hline & -2.167901 & 3875 & -1 \\
\hline & -0.423670 & 870 & \\
\hline & 0.532824 & 416 & 0.904 \\
\hline & 0.176172 & -0.839192 & -0.2 \\
\hline & 0.459264 & 1.297197 & -1.0 \\
\hline & 0.516954 & 1.418296 & 0.3 \\
\hline & 0.528380 & 363 & \\
\hline & 0.505890 & 3.825073 & \\
\hline & 0.462844 & 3.684735 & -1.32853 \\
\hline & -1.276147 & -1.106091 & \\
\hline & -3.8 & -0.2 & \\
\hline & -1.863848 & -1.6 & \\
\hline & -0.0 & -0.3 & \\
\hline & -1.9 & -1.7 & \\
\hline & 0.3764 & 2.3 & -3.0 \\
\hline & & -2.3 & \\
\hline & & -1.9 & \\
\hline & -4.2 & & \\
\hline & -6.4 & -1.1 & -0. \\
\hline & -6.4 & & -1 \\
\hline & -5.6 & & \\
\hline & -4.8 & 1.4 & -0 . \\
\hline & -0. & -1. & \\
\hline & -0.7 & & \\
\hline & & -1 & -0 . \\
\hline & & & \\
\hline & & & \\
\hline & 0 & & -1. \\
\hline & 3.0 & -2.5 & \\
\hline & & -1.8 & -1.2 \\
\hline & & 0.5 & \\
\hline & 2.4 & -0.4 & \\
\hline & & -1.8 & \\
\hline & & -1.6 & -0.1 \\
\hline & 3.3 & -0.3 & \\
\hline & 3.842223 & 0.7 & -0.3 \\
\hline & 4.61 & 0.5 & -1.46274 \\
\hline & 4.850815 & -0.72 & -1.93940 \\
\hline & 3.072101 & -3.524513 & 0.67547 \\
\hline & 4.541509 & -2.84 & -1.63623 \\
\hline & 2.4431 & 1.57 & 2.39344 \\
\hline & 1.878381 & 0.321957 & 3.51620 \\
\hline & 1.992531 & -2.384995 & 2.63267 \\
\hline & 3.6553 & 1.798087 & 0.04178 \\
\hline & 5.028020 & 1.428694 & -1.99518 \\
\hline & & -0.861390 & -2.8 \\
\hline & 3.602236 & 0.470984 & $31100 ?$ \\
\hline
\end{tabular}

Figure S23B Radical C3 Product 


\begin{tabular}{|c|c|c|c|}
\hline & & & \\
\hline & 4 & & \\
\hline & & & \\
\hline & & & \\
\hline & & & \\
\hline & & & \\
\hline & & & \\
\hline & & & \\
\hline & & & \\
\hline & & & \\
\hline & -2.1 & & \\
\hline & -0.5 & & \\
\hline & 0.6 & & \\
\hline & & & \\
\hline & & & \\
\hline & & & \\
\hline & & & \\
\hline & & & \\
\hline & & & \\
\hline & -1.3 & & \\
\hline & $-3 . c$ & & \\
\hline & & & \\
\hline & & & \\
\hline & & & \\
\hline & & & \\
\hline & -3.6 & & \\
\hline & $-4 . \varepsilon$ & -1 & \\
\hline & -4.4 & & \\
\hline & & & \\
\hline & & & \\
\hline & & & \\
\hline & & & \\
\hline & & & \\
\hline & & & \\
\hline & & & \\
\hline & & & \\
\hline & & & \\
\hline & & & \\
\hline & & & \\
\hline & & & \\
\hline & & & \\
\hline C & & & \\
\hline C & & & \\
\hline C & & & \\
\hline 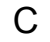 & & & \\
\hline C & & & \\
\hline 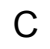 & & -0 . & \\
\hline C & & & \\
\hline $\mathrm{H}$ & & & \\
\hline & & -3 & \\
\hline & 2.9 & & \\
\hline & & & \\
\hline & & & \\
\hline & & & \\
\hline & 50 & 0.591432 & -1.9 \\
\hline
\end{tabular}

$\begin{array}{llll}\text { H } & 5.773491 & -1.822721 & -2.318388\end{array}$

H $3.416936 \quad-0.465251 \quad 2.909292$

Figure S23B Radical C4 TS

02

N $\quad 4.424266 \quad-2.387935 \quad-0.475959$

C $3.829139-0.678768 \quad-2.227623$

$\begin{array}{llll}\text { C } & 3.570014 & -0.895868 & 2.811526\end{array}$

$\begin{array}{llll}\text { C } & 3.761834 & -1.267700 & 1.369207\end{array}$

$\begin{array}{llll}\text { C } & 4.373517 & -2.414673 & 0.901565\end{array}$

C $3.870874-1.208372 \quad-0.927611$

$\begin{array}{llll}\text { C } & 3.418715 & -0.493148 & 0.209947\end{array}$

$\begin{array}{llll}\text { C } & 2.756752 & 0.777899 & 0.033154\end{array}$

C $2.884204 \quad 1.364187 \quad-1.276345$

$\begin{array}{llll}\text { C } & 3.348154 & 0.634221 & -2.366135\end{array}$

H $4.873999 \quad-3.081719-1.054529$

H $\quad 4.204861 \quad-1.236537 \quad-3.080288$

$\begin{array}{llll}\mathrm{H} & 2.512983 & -0.770922 & 3.075051\end{array}$

H $3.985680 \quad-1.664654 \quad 3.471261$

H 4.786768 -3.248643 1.452181

$\begin{array}{llll}H & 2.747938 & 1.457244 & 0.882528\end{array}$

H $\quad 2.531591 \quad 2.379965-1.425442$

$\begin{array}{llll}H & 3.358867 & 1.097370 & -3.349539\end{array}$

N $\quad-0.357798 \quad 1.494762 \quad-1.668088$

C $\quad-2.159326-1.158956 \quad 1.376040$

$\begin{array}{llll}\text { O } & -2.162976 & -0.887492 & 2.576758\end{array}$

$\begin{array}{llll}\text { C } & -0.876004 & -1.327171 & 0.545227\end{array}$

C $\quad-1.067118 \quad 3.656173 \quad-0.596666$

C $\quad-3.265338-1.654156-0.800889$

C $\quad-4.698325-1.316620-1.236205$

$\begin{array}{llll}\text { C } & -5.532464 & -1.648771 & 0.014053\end{array}$

$\begin{array}{llll}\text { C } & -4.636573 & -1.216548 & 1.186741\end{array}$

C $\quad-2.174469-0.846766-1.513142$

$\begin{array}{llll}\text { C } & 0.379959 & -0.651770 & 1.144703\end{array}$

$\begin{array}{llll}\text { C } & 0.672125 & 0.528246 & 0.234648\end{array}$

C $\quad 0.071165 \quad 0.212965 \quad-1.141978$

$\begin{array}{llll}\text { C } & -0.476288 & 2.393858 & -0.607098\end{array}$

C $\quad 0.145545 \quad 1.863390 \quad 0.548933$

C $\quad 0.185985 \quad 2.619626 \quad 1.722579$

C $\quad-0.400903 \quad 3.890530 \quad 1.740413$

C $\quad-1.019562 \quad 4.397864 \quad 0.591460$

$\begin{array}{llll}\mathrm{N} & -1.045890 & -0.707738 & -0.783066\end{array}$

$\begin{array}{llll}N & -3.277668 & -1.368299 & 0.646857\end{array}$

$\begin{array}{llll}\mathrm{O} & -2.339530 & -0.363627 & -2.637887\end{array}$

$\begin{array}{llll}\mathrm{H} & -1.077210 & 1.455556 & -2.382079\end{array}$

$\begin{array}{llll}\mathrm{H} & -0.710920 & -2.408486 & 0.429422\end{array}$

H $\quad-1.555904 \quad 4.052243 \quad-1.482637$

H $\quad-3.059256 \quad-2.723719 \quad-0.968918$

H $\quad-5.000693 \quad-1.880290 \quad-2.121499$

H $\quad-4.761491 \quad-0.250784 \quad-1.478499$

$\begin{array}{llll}\mathrm{H} & -5.722245 & -2.726957 & 0.065748\end{array}$

H $\quad-6.499070 \quad-1.138266 \quad 0.025124$

H $\quad-4.761781 \quad-1.833040 \quad 2.081638$

H $\quad-4.805484-0.171172 \quad 1.473151$

H $\quad 1.207346 \quad-1.366087 \quad 1.146789$ 


$\begin{array}{rrrr}\mathrm{H} & 0.181184 & -0.351989 & 2.176408 \\ \mathrm{H} & 0.743163 & -0.291778 & -1.842301 \\ \mathrm{H} & 0.655124 & 2.219821 & 2.618542 \\ \mathrm{H} & -0.380090 & 4.483189 & 2.650543 \\ \mathrm{H} & -1.476373 & 5.383781 & 0.618164 \\ \mathrm{H} & 4.071011 & 0.051272 & 3.050982\end{array}$

Figure S23B Radical C4 Product

\begin{tabular}{lrrr} 
O 2 & & & \\
$\mathrm{N}$ & 0.429591 & 1.847117 & -1.472722 \\
$\mathrm{C}$ & -2.415722 & -0.558557 & 1.366240 \\
$\mathrm{O}$ & -2.556133 & -0.519859 & 2.589808 \\
$\mathrm{C}$ & -1.036369 & -0.633979 & 0.712787 \\
$\mathrm{C}$ & 0.959831 & 4.131102 & -0.579543 \\
$\mathrm{C}$ & -3.311789 & -0.588914 & -0.969665 \\
$\mathrm{C}$ & -4.609809 & 0.071985 & -1.454506 \\
$\mathrm{C}$ & -5.633458 & -0.379319 & -0.397447 \\
$\mathrm{C}$ & -4.844692 & -0.368508 & 0.925188 \\
$\mathrm{C}$ & -2.026912 & 0.067444 & -1.469099 \\
$\mathrm{C}$ & 0.065421 & 0.081498 & 1.502320 \\
$\mathrm{C}$ & 1.114058 & 0.552701 & 0.448042 \\
$\mathrm{C}$ & 0.359624 & 0.504398 & -0.931188 \\
$\mathrm{C}$ & 0.895394 & 2.736861 & -0.512551 \\
$\mathrm{C}$ & 1.392779 & 2.042567 & 0.604859 \\
$\mathrm{C}$ & 1.973186 & 2.736980 & 1.658052 \\
$\mathrm{C}$ & 2.056489 & 4.136882 & 1.598651 \\
$\mathrm{C}$ & 1.546913 & 4.818097 & 0.489617 \\
$\mathrm{~N}$ & -1.004338 & 0.051684 & -0.586415 \\
$\mathrm{~N}$ & -3.447917 & -0.544599 & 0.495228 \\
$\mathrm{O}$ & -1.948271 & 0.555433 & -2.600695 \\
$\mathrm{H}$ & -0.272844 & 2.105200 & -2.153858 \\
$\mathrm{H}$ & -0.780719 & -1.696406 & 0.578690 \\
$\mathrm{H}$ & 0.568745 & 4.666870 & -1.440142 \\
$\mathrm{H}$ & -3.284237 & -1.637620 & -1.309176 \\
$\mathrm{H}$ & -4.869931 & -0.232050 & -2.470414 \\
$\mathrm{H}$ & -4.490828 & 1.160988 & -1.445471 \\
$\mathrm{H}$ & -5.978583 & -1.395307 & -0.620109 \\
$\mathrm{H}$ & -6.512517 & 0.269025 & -0.355217 \\
$\mathrm{H}$ & -5.140368 & -1.168554 & 1.610655 \\
$\mathrm{H}$ & -4.940779 & 0.582463 & 1.462020 \\
$\mathrm{H}$ & 0.497702 & -0.551977 & 2.273595 \\
$\mathrm{H}$ & -0.369058 & 0.948983 & 2.004822 \\
$\mathrm{H}$ & 0.790957 & -0.213523 & -1.634662 \\
$\mathrm{H}$ & 2.353011 & 2.205306 & 2.527855 \\
$\mathrm{H}$ & 2.510188 & 4.688718 & 2.416725 \\
$\mathrm{H}$ & 1.605630 & 5.902985 & 0.451149 \\
$\mathrm{~N}$ & 2.237110 & -3.722913 & -0.951601 \\
$\mathrm{C}$ & 3.197737 & -1.704071 & -2.077442 \\
$\mathrm{C}$ & 1.481124 & -2.842750 & 2.554984 \\
$\mathrm{C}$ & 1.812794 & -2.863470 & 1.085847 \\
$\mathrm{C}$ & 2.538940 & -4.023461 & 0.319736 \\
\hline
\end{tabular}

$\begin{array}{lrrr}\mathrm{C} & 3.651610 & -0.390425 & -1.800823 \\ \mathrm{H} & 2.408747 & -4.397622 & -1.682658 \\ \mathrm{H} & 3.429284 & -2.199806 & -3.014666 \\ \mathrm{H} & 0.422213 & -2.637864 & 2.757524 \\ \mathrm{H} & 1.708829 & -3.812705 & 3.010419 \\ \mathrm{H} & 1.572271 & -5.037227 & 0.600879 \\ \mathrm{H} & 2.887779 & -0.148904 & 1.437860 \\ \mathrm{H} & 3.754970 & 1.248708 & -0.446791 \\ \mathrm{H} & 4.254678 & 0.120049 & -2.548351 \\ \mathrm{H} & 2.067251 & -2.086215 & 3.089210\end{array}$

Figure S23B Radical C5 TS

02

$\begin{array}{lrrr}\text { N } & -0.101160 & 1.075050 & -1.595294 \\ \mathrm{C} & -2.634826 & -0.841903 & 1.480686 \\ \mathrm{O} & -3.027726 & -0.244309 & 2.482553 \\ \mathrm{C} & -1.148922 & -1.052269 & 1.143191 \\ \mathrm{C} & -1.143840 & 3.359616 & -1.466511 \\ \mathrm{C} & -2.974536 & -2.090830 & -0.647401 \\ \mathrm{C} & -4.215547 & -2.114021 & -1.550507 \\ \mathrm{C} & -5.379202 & -2.195177 & -0.545996 \\ \mathrm{C} & -4.921748 & -1.313823 & 0.627976 \\ \mathrm{C} & -1.762490 & -1.364260 & -1.240176 \\ \mathrm{C} & -0.179067 & -0.064729 & 1.833040 \\ \mathrm{C} & 0.365737 & 0.814962 & 0.715844 \\ \mathrm{C} & 0.206899 & 0.056678 & -0.608475 \\ \mathrm{C} & -0.541743 & 2.221640 & -0.932756 \\ \mathrm{C} & -0.260862 & 2.120049 & 0.450754 \\ \mathrm{C} & -0.573972 & 3.181622 & 1.301847 \\ \mathrm{C} & -1.173034 & 4.331763 & 0.772882 \\ \mathrm{C} & -1.453912 & 4.413656 & -0.595887 \\ \mathrm{~N} & -0.925180 & -0.865815 & -0.303800 \\ \mathrm{~N} & -3.456099 & -1.409659 & 0.570012 \\ \mathrm{O} & -1.594514 & -1.242881 & -2.457900 \\ \mathrm{H} & -0.584483 & 0.747419 & -2.424483 \\ \mathrm{H} & -0.903387 & -2.087792 & 1.422180 \\ \mathrm{H} & -1.371981 & 3.426689 & -2.526829 \\ \mathrm{H} & -2.664056 & -3.119299 & -0.401385 \\ \mathrm{H} & -4.193147 & -2.947985 & -2.255453 \\ \mathrm{H} & -4.259859 & -1.185688 & -2.129262 \\ \mathrm{H} & -5.513833 & -3.228780 & -0.207261 \\ \mathrm{H} & -6.328048 & -1.855970 & -0.969881 \\ \mathrm{H} & -5.287602 & -1.657666 & 1.599832 \\ \mathrm{H} & -5.229233 & -0.268010 & 0.504837 \\ \mathrm{H} & 0.615116 & -0.628748 & 2.329375 \\ \mathrm{H} & -0.714090 & 0.509146 & 2.593439 \\ \mathrm{H} & 1.075180 & -0.535146 & -0.914242 \\ \mathrm{H} & -0.369075 & 3.111510 & 2.367662 \\ \mathrm{H} & -1.425709 & 5.160453 & 1.428234 \\ \mathrm{H} & -1.925214 & 5.308032 & -0.995249 \\ \mathrm{~N} & 4.964346 & -0.875054 & -1.497722 \\ \mathrm{C} & 3.665182 & 1.247005 & -1.033154 \\ \mathrm{C} & 4.468458 & -3.085458 & 1.449847 \\ \mathrm{C} & 4.499768 & -2.018875 & 0.396870 \\ \mathrm{C} & 5.184403 & -2.047829 & -0.792547\end{array}$




$\begin{array}{lrrr}\mathrm{C} & 4.128358 & -0.061665 & -0.772783 \\ \mathrm{C} & 3.803362 & -0.750726 & 0.431810 \\ \mathrm{C} & 2.982992 & -0.127564 & 1.378388 \\ \mathrm{C} & 2.357267 & 1.128773 & 1.069481 \\ \mathrm{C} & 2.833059 & 1.835002 & -0.111928 \\ \mathrm{H} & 5.361869 & -0.656211 & -2.398983 \\ \mathrm{H} & 3.975205 & 1.780143 & -1.928220 \\ \mathrm{H} & 3.445220 & -3.433148 & 1.644847 \\ \mathrm{H} & 5.063246 & -3.954468 & 1.149868 \\ \mathrm{H} & 5.818102 & -2.821139 & -1.205153 \\ \mathrm{H} & 2.800044 & -0.600818 & 2.338799 \\ \mathrm{H} & 2.094523 & 1.756385 & 1.920948 \\ \mathrm{H} & 2.467255 & 2.841954 & -0.287431 \\ \mathrm{H} & 4.867837 & -2.724811 & 2.407226\end{array}$

Figure S23B Radical C5 Product

$\begin{array}{lrrr}02 & & & \\ \mathrm{~N} & -0.119646 & 1.561815 & -1.593199 \\ \mathrm{C} & -2.566201 & -0.986770 & 1.471354 \\ \mathrm{O} & -2.933168 & -0.710083 & 2.613940 \\ \mathrm{C} & -1.125206 & -0.772092 & 1.000120 \\ \mathrm{C} & -0.637102 & 3.996132 & -1.256340 \\ \mathrm{C} & -2.977170 & -1.818510 & -0.850751 \\ \mathrm{C} & -4.302269 & -1.755607 & -1.623986 \\ \mathrm{C} & -5.323195 & -2.264699 & -0.591097 \\ \mathrm{C} & -4.828200 & -1.685967 & 0.746572 \\ \mathrm{C} & -1.883920 & -0.887723 & -1.371243 \\ \mathrm{C} & -0.394923 & 0.368412 & 1.723824 \\ \mathrm{C} & 0.544111 & 1.032546 & 0.665291 \\ \mathrm{C} & 0.121809 & 0.429676 & -0.719717 \\ \mathrm{C} & -0.223348 & 2.728643 & -0.839826 \\ \mathrm{C} & 0.217889 & 2.507042 & 0.476507 \\ \mathrm{C} & 0.266436 & 3.557694 & 1.383937 \\ \mathrm{C} & -0.135379 & 4.838958 & 0.976396 \\ \mathrm{C} & -0.585870 & 5.045234 & -0.330731 \\ \mathrm{~N} & -1.079422 & -0.373706 & -0.413784 \\ \mathrm{~N} & -3.383874 & -1.504345 & 0.528862 \\ \mathrm{O} & -1.757492 & -0.647443 & -2.575662 \\ \mathrm{H} & -0.724940 & 1.392185 & -2.387333 \\ \mathrm{H} & -0.579287 & -1.719172 & 1.134956 \\ \mathrm{H} & -0.987019 & 4.163551 & -2.271307 \\ \mathrm{H} & -2.571036 & -2.842574 & -0.895829 \\ \mathrm{H} & -4.272245 & -2.352491 & -2.537757 \\ \mathrm{H} & -4.512438 & -0.717494 & -1.903564 \\ \mathrm{H} & -5.306588 & -3.359902 & -0.555162 \\ \mathrm{H} & -6.346269 & -1.954030 & -0.818353 \\ \mathrm{H} & -5.008144 & -2.348846 & 1.598187 \\ \mathrm{H} & -5.285138 & -0.716118 & 0.976168 \\ \mathrm{H} & 0.149428 & 0.000561 & 2.596088 \\ \mathrm{H} & -1.129246 & 1.091080 & 2.085058 \\ \mathrm{H} & 0.881610 & -0.231545 & -1.148905 \\ \mathrm{H} & 0.601286 & 3.390224 & 2.405337 \\ \mathrm{H} & -0.102490 & 5.666817 & 1.678685 \\ \mathrm{H} & -0.904798 & 6.037662 & -0.639278 \\ \mathrm{~N} & 4.877130 & -1.518144 & -1.253233\end{array}$

$\begin{array}{lrrr}\mathrm{C} & 3.870665 & 0.770679 & -0.813812 \\ \mathrm{C} & 3.307284 & -3.857672 & 1.168853 \\ \mathrm{C} & 3.775887 & -2.723282 & 0.309042 \\ \mathrm{C} & 4.717496 & -2.773547 & -0.690004 \\ \mathrm{C} & 4.036271 & -0.631718 & -0.626503 \\ \mathrm{C} & 3.315358 & -1.349403 & 0.368499 \\ \mathrm{C} & 2.391184 & -0.693061 & 1.177647 \\ \mathrm{C} & 2.080935 & 0.773358 & 0.984825 \\ \mathrm{C} & 2.968760 & 1.437387 & -0.047111 \\ \mathrm{H} & 5.511997 & -1.295703 & -2.005333 \\ \mathrm{H} & 4.471528 & 1.300573 & -1.549658 \\ \mathrm{H} & 2.226988 & -4.027615 & 1.064732 \\ \mathrm{H} & 3.816646 & -4.790674 & 0.905935 \\ \mathrm{H} & 5.294901 & -3.612757 & -1.053355 \\ \mathrm{H} & 1.888266 & -1.229156 & 1.977190 \\ \mathrm{H} & 2.224874 & 1.299173 & 1.948590 \\ \mathrm{H} & 2.845495 & 2.508363 & -0.176231 \\ \mathrm{H} & 3.494781 & -3.665491 & 2.233899\end{array}$

Figure S23B Radical C6 TS 02

$\begin{array}{lrrr}\text { N } & 0.265708 & 0.489845 & -1.431330 \\ \mathrm{C} & -3.102061 & -0.299133 & 1.399508 \\ \mathrm{O} & -3.569103 & 0.553118 & 2.155126 \\ \mathrm{C} & -1.626946 & -0.733673 & 1.418794 \\ \mathrm{C} & -0.303932 & 2.861026 & -2.044520 \\ \mathrm{C} & -3.256682 & -1.963198 & -0.448991 \\ \mathrm{C} & -4.309087 & -2.047212 & -1.563253 \\ \mathrm{C} & -5.628170 & -1.769166 & -0.820289 \\ \mathrm{C} & -5.252241 & -0.703634 & 0.222744 \\ \mathrm{C} & -1.859650 & -1.542618 & -0.916885 \\ \mathrm{C} & -0.658815 & 0.290992 & 2.059553 \\ \mathrm{C} & 0.237476 & 0.763580 & 0.922416 \\ \mathrm{C} & 0.185109 & -0.276263 & -0.201179 \\ \mathrm{C} & -0.049512 & 1.820228 & -1.152825 \\ \mathrm{C} & -0.055024 & 2.034553 & 0.246374 \\ \mathrm{C} & -0.303496 & 3.312377 & 0.751533 \\ \mathrm{C} & -0.553996 & 4.364950 & -0.137298 \\ \mathrm{C} & -0.554612 & 4.135698 & -1.518381 \\ \mathrm{~N} & -1.135600 & -0.922788 & 0.040417 \\ \mathrm{~N} & -3.829957 & -0.971780 & 0.481196 \\ \mathrm{O} & -1.458994 & -1.748919 & -2.066965 \\ \mathrm{H} & -0.111076 & 0.036184 & -2.256286 \\ \mathrm{H} & -1.583391 & -1.690948 & 1.959163 \\ \mathrm{H} & -0.314333 & 2.688517 & -3.117350 \\ \mathrm{H} & -3.161243 & -2.938052 & 0.056518 \\ \mathrm{H} & -4.292303 & -3.014701 & -2.069735 \\ \mathrm{H} & -4.108421 & -1.272667 & -2.310731 \\ \mathrm{H} & -5.976438 & -2.678506 & -0.317294 \\ \mathrm{H} & -6.425347 & -1.429515 & -1.486781 \\ \mathrm{H} & -5.828344 & -0.778003 & 1.149628 \\ \mathrm{H} & -5.371031 & 0.315547 & -0.165134 \\ \mathrm{H} & -0.094538 & -0.195297 & 2.859205 \\ \mathrm{H} & -1.231016 & 1.109055 & 2.503659 \\ \mathrm{H} & 0.967053 & -1.041636 & -0.163663\end{array}$




$\begin{array}{rrrr}\mathrm{H} & -0.316232 & 3.486458 & 1.825023 \\ \mathrm{H} & -0.753941 & 5.361395 & 0.246319 \\ \mathrm{H} & -0.756964 & 4.958333 & -2.199461 \\ \mathrm{~N} & 4.612971 & 0.341156 & -1.222571 \\ \mathrm{C} & 3.001693 & 1.230941 & 0.487554 \\ \mathrm{C} & 5.364913 & -3.165980 & -0.245745 \\ \mathrm{C} & 4.885589 & -1.753666 & -0.409610 \\ \mathrm{C} & 5.248712 & -0.871348 & -1.406146 \\ \mathrm{C} & 3.807945 & 0.267939 & -0.101945 \\ \mathrm{C} & 3.969451 & -1.046907 & 0.435380 \\ \mathrm{C} & 3.288990 & -1.369186 & 1.637875 \\ \mathrm{C} & 2.470190 & -0.435282 & 2.228870 \\ \mathrm{C} & 2.195451 & 0.850824 & 1.614223 \\ \mathrm{H} & 4.686095 & 1.135361 & -1.840357 \\ \mathrm{H} & 2.917798 & 2.229792 & 0.070476 \\ \mathrm{H} & 4.533512 & -3.882529 & -0.266057 \\ \mathrm{H} & 6.059179 & -3.441529 & -1.046287 \\ \mathrm{H} & 5.921990 & -1.016140 & -2.240212 \\ \mathrm{H} & 3.444542 & -2.336684 & 2.109677 \\ \mathrm{H} & 1.995766 & -0.670620 & 3.177130 \\ \mathrm{H} & 1.897289 & 1.653397 & 2.287298 \\ \mathrm{H} & 5.886538 & -3.310882 & 0.709442\end{array}$

Figure S23B Radical C6 Product

02

$\begin{array}{rrrr}\text { N } & 0.098901 & 1.051300 & -1.574953 \\ \mathrm{C} & -3.065573 & -0.584440 & 1.445621 \\ \mathrm{O} & -3.536363 & -0.054982 & 2.452857 \\ \mathrm{C} & -1.558598 & -0.638766 & 1.176721 \\ \mathrm{C} & -0.102977 & 3.550690 & -1.712833 \\ \mathrm{C} & -3.278238 & -1.784768 & -0.736549 \\ \mathrm{C} & -4.466217 & -1.720217 & -1.707018 \\ \mathrm{C} & -5.677065 & -1.903360 & -0.774946 \\ \mathrm{C} & -5.288394 & -1.140623 & 0.504459 \\ \mathrm{C} & -2.005637 & -1.099597 & -1.230571 \\ \mathrm{C} & -0.767619 & 0.515645 & 1.809384 \\ \mathrm{C} & 0.387181 & 0.857149 & 0.812919 \\ \mathrm{C} & 0.063664 & 0.071998 & -0.505960 \\ \mathrm{C} & 0.068491 & 2.337354 & -1.041991 \\ \mathrm{C} & 0.303493 & 2.303019 & 0.343569 \\ \mathrm{C} & 0.386173 & 3.484933 & 1.068556 \\ \mathrm{C} & 0.226188 & 4.711746 & 0.406382 \\ \mathrm{C} & -0.020450 & 4.734052 & -0.969459 \\ \mathrm{~N} & -1.268575 & -0.515932 & -0.258831 \\ \mathrm{~N} & -3.816917 & -1.169769 & 0.487644 \\ \mathrm{O} & -1.689917 & -1.100503 & -2.424007 \\ \mathrm{H} & -0.416806 & 0.815927 & -2.414236 \\ \mathrm{H} & -1.184677 & -1.605931 & 1.548713 \\ \mathrm{H} & -0.293906 & 3.575357 & -2.782212 \\ \mathrm{H} & -3.016797 & -2.837888 & -0.540819 \\ \mathrm{H} & -4.398846 & -2.480233 & -2.488047 \\ \mathrm{H} & -4.489185 & -0.737588 & -2.190666 \\ \mathrm{H} & -5.819775 & -2.966234 & -0.549416 \\ \mathrm{H} & -6.606690 & -1.527118 & -1.209823 \\ \mathrm{H} & -5.671148 & -1.604733 & 1.418309\end{array}$

$\begin{array}{rrrr}\mathrm{H} & -5.631258 & -0.099353 & 0.489796 \\ \mathrm{H} & -0.401506 & 0.248634 & 2.803109 \\ \mathrm{H} & -1.427869 & 1.376228 & 1.933279 \\ \mathrm{H} & 0.770914 & -0.739261 & -0.706337 \\ \mathrm{H} & 0.561846 & 3.463945 & 2.141964 \\ \mathrm{H} & 0.287716 & 5.641520 & 0.964383 \\ \mathrm{H} & -0.152437 & 5.686520 & -1.476646 \\ \mathrm{~N} & 4.827437 & -0.008359 & -0.944487 \\ \mathrm{C} & 2.918302 & 0.852849 & 0.423305 \\ \mathrm{C} & 5.141064 & -3.633303 & -0.185239 \\ \mathrm{C} & 4.788338 & -2.179008 & -0.302936 \\ \mathrm{C} & 5.424120 & -1.244193 & -1.105212 \\ \mathrm{C} & 3.781910 & -0.114483 & -0.040617 \\ \mathrm{C} & 3.749720 & -1.484223 & 0.376989 \\ \mathrm{C} & 2.773378 & -1.867126 & 1.354234 \\ \mathrm{C} & 1.887564 & -0.960743 & 1.840349 \\ \mathrm{C} & 1.818897 & 0.484585 & 1.389363 \\ \mathrm{H} & 5.088543 & 0.832082 & -1.437509 \\ \mathrm{H} & 2.986814 & 1.882100 & 0.085806 \\ \mathrm{H} & 4.306991 & -4.280063 & -0.486891 \\ \mathrm{H} & 5.998766 & -3.882851 & -0.818355 \\ \mathrm{H} & 6.260925 & -1.369627 & -1.778436 \\ \mathrm{H} & 2.758423 & -2.890319 & 1.724470 \\ \mathrm{H} & 1.185055 & -1.272397 & 2.608673 \\ \mathrm{H} & 1.903544 & 1.126221 & 2.287369 \\ \mathrm{H} & 5.399407 & -3.906765 & 0.846009\end{array}$

Figure S23B Radical C7 TS 


$\begin{array}{rrrr}\mathrm{H} & -4.250300 & -0.004208 & -2.452854 \\ \mathrm{H} & -6.531190 & -1.158601 & -0.748981 \\ \mathrm{H} & -6.545317 & 0.370099 & -1.638681 \\ \mathrm{H} & -5.895747 & 0.285297 & 1.064977 \\ \mathrm{H} & -5.091386 & 1.436504 & -0.012380 \\ \mathrm{H} & -0.287220 & -1.143139 & 2.730313 \\ \mathrm{H} & -1.003585 & 0.471491 & 2.670054 \\ \mathrm{H} & 0.588750 & -1.675937 & -0.396137 \\ \mathrm{H} & 0.564379 & 2.596983 & 2.402858 \\ \mathrm{H} & 0.767270 & 4.766195 & 1.195729 \\ \mathrm{H} & 0.740351 & 4.822866 & -1.282063 \\ \mathrm{~N} & 3.611321 & 1.071033 & 0.338382 \\ \mathrm{C} & 2.235848 & -0.545954 & 1.722043 \\ \mathrm{C} & 5.582662 & -0.930910 & -2.090340 \\ \mathrm{C} & 4.686470 & -0.368282 & -1.027344 \\ \mathrm{C} & 4.502335 & 0.956822 & -0.716133 \\ \mathrm{C} & 3.193768 & -0.177239 & 0.717077 \\ \mathrm{C} & 3.853323 & -1.114263 & -0.114565 \\ \mathrm{C} & 3.640290 & -2.493985 & 0.096675 \\ \mathrm{C} & 2.805008 & -2.889492 & 1.149947 \\ \mathrm{C} & 2.174155 & -1.957958 & 1.976037 \\ \mathrm{H} & 3.242430 & 1.939949 & 0.697934 \\ \mathrm{H} & 2.093083 & 0.127752 & 2.565023 \\ \mathrm{H} & 5.021293 & -1.527583 & -2.821324 \\ \mathrm{H} & 6.093569 & -0.132025 & -2.637923 \\ \mathrm{H} & 4.945505 & 1.840011 & -1.155455 \\ \mathrm{H} & 4.138162 & -3.235562 & -0.521835 \\ \mathrm{H} & 2.674044 & -3.949330 & 1.354639 \\ \mathrm{H} & 1.588711 & -2.311218 & 2.819861 \\ \mathrm{H} & 6.353788 & -1.589169 & -1.668442\end{array}$

Figure S23B Radical C7 Product

02

$\begin{array}{lrrr}\text { N } & -0.362704 & 0.719051 & 1.495497 \\ \mathrm{C} & 3.201568 & -0.191986 & -1.382528 \\ \mathrm{O} & 3.543373 & 0.383114 & -2.416451 \\ \mathrm{C} & 1.754289 & -0.622229 & -1.126212 \\ \mathrm{C} & -0.867000 & 3.177414 & 1.488855 \\ \mathrm{C} & 3.663799 & -1.163535 & 0.874552 \\ \mathrm{C} & 4.766879 & -0.727454 & 1.849619 \\ \mathrm{C} & 6.007810 & -0.642745 & 0.943242 \\ \mathrm{C} & 5.466995 & -0.090573 & -0.387866 \\ \mathrm{C} & 2.243270 & -0.810785 & 1.310440 \\ \mathrm{C} & 0.708820 & 0.252182 & -1.833110 \\ \mathrm{C} & -0.526245 & 0.320983 & -0.877039 \\ \mathrm{C} & -0.041465 & -0.275710 & 0.491478 \\ \mathrm{C} & -0.672575 & 1.930152 & 0.889578 \\ \mathrm{C} & -0.852043 & 1.757400 & -0.495193 \\ \mathrm{C} & -1.243049 & 2.828847 & -1.290417 \\ \mathrm{C} & -1.457708 & 4.083574 & -0.697873 \\ \mathrm{C} & -1.263088 & 4.246666 & 0.677283 \\ \mathrm{~N} & 1.404759 & -0.500098 & 0.295569 \\ \mathrm{~N} & 4.054412 & -0.504466 & -0.382832 \\ \mathrm{O} & 1.905802 & -0.825772 & 2.497499 \\ \mathrm{H} & 0.157343 & 0.672499 & 2.362990\end{array}$

$\begin{array}{rrrr}\mathrm{H} & 1.651685 & -1.671791 & -1.443680 \\ \mathrm{H} & -0.720895 & 3.311786 & 2.556904 \\ \mathrm{H} & 3.693599 & -2.258234 & 0.746783 \\ \mathrm{H} & 4.880726 & -1.427762 & 2.679516 \\ \mathrm{H} & 4.518201 & 0.254884 & 2.265795 \\ \mathrm{H} & 6.430140 & -1.642395 & 0.790885 \\ \mathrm{H} & 6.794215 & -0.008465 & 1.360424 \\ \mathrm{H} & 5.982040 & -0.493837 & -1.264833 \\ \mathrm{H} & 5.524902 & 1.003018 & -0.440344 \\ \mathrm{H} & 0.457222 & -0.145135 & -2.818855 \\ \mathrm{H} & 1.123314 & 1.250401 & -1.986550 \\ \mathrm{H} & -0.513643 & -1.232882 & 0.735495 \\ \mathrm{H} & -1.373449 & 2.703287 & -2.363136 \\ \mathrm{H} & -1.764204 & 4.926922 & -1.309454 \\ \mathrm{H} & -1.420976 & 5.222280 & 1.129791 \\ \mathrm{~N} & -3.683986 & 0.624760 & -0.137445 \\ \mathrm{C} & -1.774837 & -0.464792 & -1.467583 \\ \mathrm{C} & -5.652408 & -2.021817 & 1.553105 \\ \mathrm{C} & -4.699766 & -1.157507 & 0.780485 \\ \mathrm{C} & -4.721926 & 0.213803 & 0.679632 \\ \mathrm{C} & -2.972690 & -0.463597 & -0.563089 \\ \mathrm{C} & -3.584898 & -1.602708 & -0.018820 \\ \mathrm{C} & -3.101294 & -2.901509 & -0.345515 \\ \mathrm{C} & -2.032672 & -2.998430 & -1.263092 \\ \mathrm{C} & -1.423379 & -1.895110 & -1.818288 \\ \mathrm{H} & -3.443338 & 1.585701 & -0.335334 \\ \mathrm{H} & -2.012358 & 0.082394 & -2.398127 \\ \mathrm{H} & -5.136082 & -2.614338 & 2.320025 \\ \mathrm{H} & -6.411282 & -1.415688 & 2.059158 \\ \mathrm{H} & -5.393928 & 0.936542 & 1.121045 \\ \mathrm{H} & -3.560672 & -3.792950 & 0.069669 \\ \mathrm{H} & -1.687158 & -3.986279 & -1.561264 \\ \mathrm{H} & -0.637467 & -2.042771 & -2.552896 \\ \mathrm{H} & -6.177008 & -2.733859 & 0.902216\end{array}$

Figure S23D Cation C2 TS 11

$\begin{array}{lrrr}\text { N } & 2.499976 & -0.990244 & 1.891234 \\ \mathrm{C} & 3.435639 & -3.004202 & 0.701729 \\ \mathrm{C} & 3.577696 & 1.963163 & -0.087487 \\ \mathrm{C} & 3.256661 & 0.622452 & 0.476312 \\ \mathrm{C} & 2.484858 & 0.372334 & 1.636940 \\ \mathrm{C} & 3.183815 & -1.638333 & 0.889507 \\ \mathrm{C} & 3.662650 & -0.646611 & -0.020789 \\ \mathrm{C} & 4.413694 & -1.049876 & -1.150710 \\ \mathrm{C} & 4.665976 & -2.395706 & -1.332210 \\ \mathrm{C} & 4.180834 & -3.360826 & -0.412445 \\ \mathrm{H} & 2.148343 & -1.428841 & 2.730211 \\ \mathrm{H} & 3.069611 & -3.748908 & 1.401218 \\ \mathrm{H} & 2.786096 & 2.314671 & -0.766134 \\ \mathrm{H} & 3.682843 & 2.716738 & 0.698181 \\ \mathrm{H} & 2.282227 & 1.067139 & 2.440126 \\ \mathrm{H} & 4.786431 & -0.313016 & -1.855637 \\ \mathrm{H} & 5.243533 & -2.727758 & -2.189123 \\ \mathrm{H} & 4.401283 & -4.409897 & -0.586235\end{array}$




$\begin{array}{rrrr}\mathrm{H} & 4.506021 & 1.930707 & -0.665024 \\ \mathrm{~N} & 0.339368 & 0.613424 & -1.506113 \\ \mathrm{C} & -2.957120 & -0.227594 & 1.460535 \\ \mathrm{O} & -3.345914 & 0.571139 & 2.310807 \\ \mathrm{C} & -1.490321 & -0.680302 & 1.356446 \\ \mathrm{C} & 0.188921 & 3.057604 & -2.004183 \\ \mathrm{C} & -3.261675 & -1.731434 & -0.509760 \\ \mathrm{C} & -4.377587 & -1.687293 & -1.563881 \\ \mathrm{C} & -5.642378 & -1.454917 & -0.718666 \\ \mathrm{C} & -5.181472 & -0.496940 & 0.392063 \\ \mathrm{C} & -1.880927 & -1.322386 & -1.025795 \\ \mathrm{C} & -0.476265 & 0.300241 & 2.003848 \\ \mathrm{C} & 0.356178 & 0.773043 & 0.828151 \\ \mathrm{C} & 0.238298 & -0.215373 & -0.326998 \\ \mathrm{C} & 0.283667 & 1.929148 & -1.169915 \\ \mathrm{C} & 0.299375 & 2.070341 & 0.258264 \\ \mathrm{C} & 0.197518 & 3.353005 & 0.844467 \\ \mathrm{C} & 0.096952 & 4.453831 & 0.018286 \\ \mathrm{C} & 0.095324 & 4.297870 & -1.392938 \\ \mathrm{~N} & -1.096746 & -0.764613 & -0.065130 \\ \mathrm{~N} & -3.748167 & -0.807273 & 0.534380 \\ \mathrm{O} & -1.520689 & -1.473789 & -2.190846 \\ \mathrm{H} & 0.125153 & 0.232310 & -2.418643 \\ \mathrm{H} & -1.412095 & -1.670367 & 1.825868 \\ \mathrm{H} & 0.173704 & 2.955172 & -3.083959 \\ \mathrm{H} & -3.171765 & -2.748738 & -0.096719 \\ \mathrm{H} & -4.413595 & -2.603043 & -2.157298 \\ \mathrm{H} & -4.201745 & -0.848667 & -2.245604 \\ \mathrm{H} & -5.982819 & -2.400018 & -0.281454 \\ \mathrm{H} & -6.467575 & -1.038923 & -1.301773 \\ \mathrm{H} & -5.698058 & -0.650937 & 1.343329 \\ \mathrm{H} & -5.301059 & 0.555521 & 0.109117 \\ \mathrm{H} & 0.102851 & -0.214479 & 2.771517 \\ \mathrm{H} & -1.007979 & 1.125549 & 2.481909 \\ \mathrm{H} & 0.988024 & -1.015051 & -0.349455 \\ \mathrm{H} & 0.202489 & 3.462863 & 1.924597 \\ \mathrm{H} & 0.017104 & 5.449191 & 0.441983 \\ \mathrm{H} & 0.010927 & 5.183501 & -2.015901\end{array}$

Figure S23D Cation C2 Product 11

$\begin{array}{lrrr}\mathrm{N} & 2.554474 & -0.993042 & 1.452018 \\ \mathrm{C} & 4.388018 & -2.482461 & 0.672844 \\ \mathrm{C} & 3.089784 & 2.228897 & -0.414888 \\ \mathrm{C} & 3.049797 & 0.848364 & 0.130171 \\ \mathrm{C} & 2.068813 & 0.353374 & 1.168725 \\ \mathrm{C} & 3.621427 & -1.296542 & 0.706688 \\ \mathrm{C} & 3.953371 & -0.164193 & -0.130867 \\ \mathrm{C} & 5.081263 & -0.240639 & -1.009408 \\ \mathrm{C} & 5.808749 & -1.397695 & -1.033949 \\ \mathrm{C} & 5.457697 & -2.506452 & -0.193212 \\ \mathrm{H} & 2.158041 & -1.602516 & 2.154145 \\ \mathrm{H} & 4.142860 & -3.329899 & 1.303178 \\ \mathrm{H} & 2.250551 & 2.403755 & -1.098097 \\ \mathrm{H} & 2.995072 & 2.969089 & 0.386605\end{array}$

$\begin{array}{rrrr}\mathrm{H} & 2.179891 & 0.972384 & 2.070681 \\ \mathrm{H} & 5.340179 & 0.601584 & -1.641696 \\ \mathrm{H} & 6.666616 & -1.493142 & -1.690514 \\ \mathrm{H} & 6.069373 & -3.402375 & -0.244115 \\ \mathrm{H} & 4.020306 & 2.410129 & -0.956619 \\ \mathrm{~N} & 0.201303 & 0.498093 & -1.561376 \\ \mathrm{C} & -2.862096 & -0.470295 & 1.470715 \\ \mathrm{O} & -3.145377 & 0.297484 & 2.388906 \\ \mathrm{C} & -1.447947 & -1.042775 & 1.260581 \\ \mathrm{C} & -0.597631 & 2.884222 & -1.685506 \\ \mathrm{C} & -3.365621 & -1.810403 & -0.567264 \\ \mathrm{C} & -4.526508 & -1.635075 & -1.556591 \\ \mathrm{C} & -5.730150 & -1.362993 & -0.636809 \\ \mathrm{C} & -5.149118 & -0.500952 & 0.496242 \\ \mathrm{C} & -1.989041 & -1.441996 & -1.125944 \\ \mathrm{C} & -0.327222 & -0.240940 & 1.943590 \\ \mathrm{C} & 0.530149 & 0.365805 & 0.800055 \\ \mathrm{C} & 0.214994 & -0.471319 & -0.481314 \\ \mathrm{C} & -0.156425 & 1.738016 & -1.020982 \\ \mathrm{C} & 0.055982 & 1.743130 & 0.368491 \\ \mathrm{C} & -0.160867 & 2.896898 & 1.113405 \\ \mathrm{C} & -0.598835 & 4.053677 & 0.457157 \\ \mathrm{C} & -0.814006 & 4.038301 & -0.926157 \\ \mathrm{~N} & -1.109714 & -1.041704 & -0.171504 \\ \mathrm{~N} & -3.740269 & -0.924423 & 0.552994 \\ \mathrm{O} & -1.718038 & -1.492948 & -2.326384 \\ \mathrm{H} & -0.262455 & 0.175547 & -2.405761 \\ \mathrm{H} & -1.459641 & -2.074466 & 1.639507 \\ \mathrm{H} & -0.768665 & 2.878818 & -2.757778 \\ \mathrm{H} & -3.317807 & -2.854771 & -0.218824 \\ \mathrm{H} & -4.656638 & -2.513045 & -2.192631 \\ \mathrm{H} & -4.325135 & -0.775632 & -2.204207 \\ \mathrm{H} & -6.115322 & -2.304923 & -0.230607 \\ \mathrm{H} & -6.551152 & -0.861210 & -1.154987 \\ \mathrm{H} & -5.633210 & -0.666059 & 1.462777 \\ \mathrm{H} & -5.201891 & 0.570920 & 0.270966 \\ \mathrm{H} & 0.251334 & -0.897688 & 2.596930 \\ \mathrm{H} & -0.760742 & 0.537183 & 2.574921 \\ \mathrm{H} & 0.926078 & -1.274185 & -0.693864 \\ \mathrm{H} & -0.006529 & 2.905454 & 2.189538 \\ \mathrm{H} & -0.780205 & 4.961182 & 1.024264 \\ \mathrm{H} & -1.161599 & 4.939391 & -1.423547\end{array}$

Figure S23D Cation C3 TS 11

$\begin{array}{lrrr}N & 0.465649 & -0.138262 & -1.452311 \\ C & -3.020731 & 0.326576 & 1.356612 \\ O & -3.203100 & 1.312602 & 2.068231 \\ C & -1.734805 & -0.517213 & 1.417682 \\ C & 0.917941 & 2.188723 & -2.235191 \\ C & -3.657099 & -1.302943 & -0.423011 \\ C & -4.683985 & -1.118653 & -1.550182 \\ C & -5.862549 & -0.432496 & -0.836938 \\ C & -5.191757 & 0.519451 & 0.166591 \\ C & -2.200459 & -1.348306 & -0.888583\end{array}$




$\begin{array}{rrrr}\mathrm{C} & -0.514906 & 0.228332 & 2.018432 \\ \mathrm{C} & 0.464403 & 0.304445 & 0.859299 \\ \mathrm{C} & 0.116246 & -0.753992 & -0.192986 \\ \mathrm{C} & 0.719866 & 1.188696 & -1.270322 \\ \mathrm{C} & 0.721316 & 1.503274 & 0.125782 \\ \mathrm{C} & 0.871360 & 2.844648 & 0.544105 \\ \mathrm{C} & 1.060790 & 3.826429 & -0.411374 \\ \mathrm{C} & 1.091250 & 3.490281 & -1.786578 \\ \mathrm{~N} & -1.326166 & -0.899850 & 0.050486 \\ \mathrm{~N} & -3.908309 & -0.143382 & 0.456035 \\ \mathrm{O} & -1.857636 & -1.743139 & -2.001353 \\ \mathrm{H} & 0.131458 & -0.546035 & -2.316022 \\ \mathrm{H} & -1.956116 & -1.417662 & 2.007000 \\ \mathrm{H} & 0.915255 & 1.951418 & -3.293842 \\ \mathrm{H} & -3.858636 & -2.237914 & 0.123981 \\ \mathrm{H} & -4.952221 & -2.069050 & -2.015908 \\ \mathrm{H} & -4.262654 & -0.469502 & -2.324727 \\ \mathrm{H} & -6.465525 & -1.175771 & -0.303697 \\ \mathrm{H} & -6.520796 & 0.099005 & -1.528595 \\ \mathrm{H} & -5.763563 & 0.655743 & 1.088508 \\ \mathrm{H} & -5.002445 & 1.510051 & -0.263478 \\ \mathrm{H} & -0.128918 & -0.314982 & 2.881045 \\ \mathrm{H} & -0.820462 & 1.220435 & 2.357250 \\ \mathrm{H} & 0.616851 & -1.721997 & -0.092429 \\ \mathrm{H} & 0.820990 & 3.100735 & 1.597491 \\ \mathrm{H} & 1.174430 & 4.863099 & -0.112689 \\ \mathrm{H} & 1.236255 & 4.281542 & -2.516506 \\ \mathrm{~N} & 2.577521 & -2.461500 & 1.181431 \\ \mathrm{C} & 3.887169 & -2.340304 & -0.980407 \\ \mathrm{C} & 2.722199 & 0.876824 & 2.671192 \\ \mathrm{C} & 2.511641 & -0.255501 & 1.700420 \\ \mathrm{C} & 2.170742 & -1.572405 & 2.100814 \\ \mathrm{C} & 3.279159 & -1.813713 & 0.155697 \\ \mathrm{C} & 3.286820 & -0.436714 & 0.469170 \\ \mathrm{C} & 3.957051 & 0.450669 & -0.378850 \\ \mathrm{C} & 4.581997 & -0.062780 & -1.516762 \\ \mathrm{C} & 4.542659 & -1.435256 & -1.816167 \\ \mathrm{H} & 2.405385 & -3.459031 & 1.228852 \\ \mathrm{H} & 3.862553 & -3.402349 & -1.202848 \\ \mathrm{H} & 2.698068 & 1.844330 & 2.163160 \\ \mathrm{H} & 1.970723 & 0.880621 & 3.466324 \\ \mathrm{H} & 1.648351 & -1.899309 & 2.989581 \\ \mathrm{H} & 4.000646 & 1.512261 & -0.157387 \\ \mathrm{H} & 5.112453 & 0.610051 & -2.183652 \\ \mathrm{H} & 5.038022 & -1.801634 & -2.709910 \\ & 3.707026 & 0.782766 & 3.143309\end{array}$

Figure S23D Cation C3 Product

$\begin{array}{lrrr}11 & & & \\ \text { N } & 0.408224 & 0.073579 & -1.522427 \\ \text { C } & -2.854963 & -0.160231 & 1.394091 \\ \text { O } & -2.947450 & 0.639026 & 2.324355 \\ \text { C } & -1.646641 & -1.096461 & 1.203386 \\ \text { C } & 0.167977 & 2.575367 & -1.580712 \\ \text { C } & -3.636092 & -1.281384 & -0.686362\end{array}$

\begin{tabular}{rrrr}
$\mathrm{C}$ & -4.683848 & -0.796182 & -1.698397 \\
$\mathrm{C}$ & -5.792396 & -0.214069 & -0.802799 \\
$\mathrm{C}$ & -5.028754 & 0.453764 & 0.352199 \\
$\mathrm{C}$ & -2.195324 & -1.276996 & -1.205047 \\
$\mathrm{C}$ & -0.373100 & -0.658629 & 1.942914 \\
$\mathrm{C}$ & 0.628587 & -0.200716 & 0.850406 \\
$\mathrm{C}$ & 0.162081 & -0.898565 & -0.475339 \\
$\mathrm{C}$ & 0.327620 & 1.345161 & -0.942956 \\
$\mathrm{C}$ & 0.468944 & 1.262655 & 0.453579 \\
$\mathrm{C}$ & 0.431641 & 2.417180 & 1.231186 \\
$\mathrm{C}$ & 0.277238 & 3.658790 & 0.601542 \\
$\mathrm{C}$ & 0.151405 & 3.728808 & -0.790044 \\
$\mathrm{~N}$ & -1.270552 & -1.151827 & -0.217895 \\
$\mathrm{~N}$ & -3.792486 & -0.339967 & 0.440849 \\
$\mathrm{O}$ & -1.912805 & -1.360959 & -2.401067 \\
$\mathrm{H}$ & -0.111757 & -0.101878 & -2.377361 \\
$\mathrm{H}$ & -1.957658 & -2.093882 & 1.545079 \\
$\mathrm{H}$ & 0.052760 & 2.634447 & -2.658907 \\
$\mathrm{H}$ & -3.872662 & -2.305143 & -0.354322 \\
$\mathrm{H}$ & -5.029841 & -1.604305 & -2.346227 \\
$\mathrm{H}$ & -4.243594 & -0.020490 & -2.333215 \\
$\mathrm{H}$ & -6.426825 & -1.019266 & -0.415907 \\
$\mathrm{H}$ & -6.434772 & 0.493062 & -1.333480 \\
$\mathrm{H}$ & -5.565007 & 0.426276 & 1.304710 \\
$\mathrm{H}$ & -4.780083 & 1.499417 & 0.134384 \\
$\mathrm{H}$ & 0.014316 & -1.494854 & 2.529826 \\
$\mathrm{H}$ & -0.614592 & 0.145234 & 2.641411 \\
$\mathrm{H}$ & 0.664440 & -1.843185 & -0.707420 \\
$\mathrm{H}$ & 0.497395 & 2.365827 & 2.314324 \\
$\mathrm{H}$ & 0.241478 & 4.565326 & 1.197410 \\
$\mathrm{H}$ & 0.024164 & 4.695768 & -1.268856 \\
$\mathrm{~N}$ & 3.279584 & -2.442758 & 0.540731 \\
$\mathrm{C}$ & 4.888867 & -1.437774 & -1.126739 \\
$\mathrm{C}$ & 2.540973 & 0.089187 & 2.592622 \\
$\mathrm{C}$ & 2.140083 & -0.547603 & 1.227210 \\
$\mathrm{C}$ & 2.338375 & -2.033147 & 1.335847 \\
$\mathrm{C}$ & 3.846976 & -1.379906 & -0.211214 \\
$\mathrm{C}$ & 3.179343 & -0.207039 & 0.162023 \\
$\mathrm{C}$ & 3.598298 & 0.998508 & -0.391706 \\
$\mathrm{C}$ & 4.643688 & 0.974359 & -1.323651 \\
$\mathrm{C}$ & 5.276941 & -0.220747 & -1.690378 \\
$\mathrm{H}$ & 3.584511 & -3.412413 & 0.473612 \\
$\mathrm{H}$ & 5.378991 & -2.370786 & -1.384382 \\
$\mathrm{H}$ & 2.498669 & 1.176147 & 2.497745 \\
\hline & 1.869928 & -0.224864 & 3.395512 \\
$\mathrm{H}$ & -130149 & -2.727410 & 1.9838961 \\
$\mathrm{H}$ & -0.907201 & -0.118850 \\
\hline
\end{tabular}

Figure S23D Cation C4 TS 11

$\begin{array}{llll}\mathrm{N} & 4.298010 & -2.367940 & -0.688516\end{array}$ $\begin{array}{llll}\text { C } & 3.702047 & -0.471728 & -2.221449\end{array}$ 


\begin{tabular}{|c|c|c|c|}
\hline & & $r$ & \\
\hline C & 52 & & \\
\hline & 446 & 6713 & \\
\hline 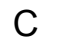 & 6986 & -1.129653 & \\
\hline 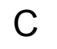 & 3.394066 & -0.513970 & \\
\hline C & 2.778545 & 0.781418 & 0.18504 \\
\hline C & 2.811338 & 1.475802 & -1.07074 \\
\hline C & 3.232684 & 0.856090 & -2.2377 \\
\hline & 4.676868 & 32490 & -1.3 \\
\hline & 4.026271 & -0.953745 & \\
\hline & 2.614417 & -0.953466 & \\
\hline & 3.971405 & & \\
\hline & 4.681324 & & \\
\hline & & & \\
\hline & & & \\
\hline & & & \\
\hline & & & \\
\hline C & -2 & & \\
\hline 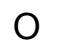 & -2.1 & & \\
\hline 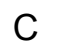 & $-0 . \varepsilon$ & & \\
\hline $\mathrm{C}$ & -1.0 & & \\
\hline $\mathrm{C}$ & -3.2 & & \\
\hline 0 & -4 & & \\
\hline C & -5.4 & & -0 . \\
\hline C & -4 & & \\
\hline C & -2 & -0 & -1 \\
\hline C & & & \\
\hline C & & & \\
\hline C & & & -1 \\
\hline C & -0.4 & 69 & -0 \\
\hline - & & & \\
\hline C & 0.1 & & \\
\hline C & -0.4 & & \\
\hline C & -1.0 & & \\
\hline$N$ & -0.9 & -0 . & -0.7 \\
\hline $\mathrm{N}$ & -3.2 & & \\
\hline 0 & -2.2 & 039 & -2.6 \\
\hline $\mathrm{H}$ & -0.8 & 175 & -2.4 \\
\hline $\mathrm{H}$ & -0.6 & 6609 & 0.4 \\
\hline $\mathrm{H}$ & -1.45 & 2295 & -1.4 \\
\hline $\mathrm{H}$ & $-2.9 \varepsilon$ & 7397 & -1.0 \\
\hline $\mathrm{H}$ & -4.9 & 6861 & -2.1 \\
\hline $\mathrm{H}$ & -4.70 & 8784 & -1.533 \\
\hline 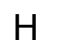 & -5.66 & -2.746368 & -0.0 \\
\hline 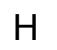 & -6.4 & -1 & -0.0 \\
\hline 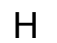 & & & \\
\hline $\mathrm{H}$ & & & \\
\hline 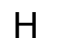 & & & \\
\hline $\mathrm{H}$ & & & \\
\hline $\mathrm{H}$ & & & \\
\hline $\mathrm{H}$ & & & \\
\hline $\mathrm{H}$ & -0.4 & & \\
\hline $\mathrm{H}$ & & & \\
\hline s & 4.265045 & -0.333897 & 3.0762 \\
\hline
\end{tabular}

Figure S23D Cation C4 Product

11

\begin{tabular}{|c|c|c|c|}
\hline & & & \\
\hline & 310 & & \\
\hline & -2.217552 & 2837 & 738 \\
\hline & -0.856208 & 8184 & 802 \\
\hline & 9151 & 75353 & 806 \\
\hline & 0174 & -1 & -0 . \\
\hline & 2477 & -1.2 & 7990 \\
\hline & 0461 & & -0. \\
\hline & -4.6 & & \\
\hline & & & \\
\hline & & & \\
\hline & & & \\
\hline & & & \\
\hline & & & \\
\hline & & & \\
\hline & & & \\
\hline & & & \\
\hline & & & \\
\hline & & & \\
\hline & & & \\
\hline & & & \\
\hline & & & \\
\hline & & & \\
\hline & & & \\
\hline & & & \\
\hline & & & \\
\hline & & -0 & \\
\hline & -5.6 & & -0 . \\
\hline & -6.4 & -1 & -0 \\
\hline & -4.8 & & 19 \\
\hline & -4.7 & $-0 .($ & 47 \\
\hline & & & \\
\hline & 0.2 & & \\
\hline & & & \\
\hline & & & \\
\hline & -0.9 & & \\
\hline & & & \\
\hline & & -1 & \\
\hline & & & \\
\hline & & & \\
\hline & & & \\
\hline & & & \\
\hline & & -0 . & \\
\hline & & & \\
\hline & & & \\
\hline & & & \\
\hline & & & \\
\hline & & -2.2 & \\
\hline & & & -2 \\
\hline & & & \\
\hline & & & \\
\hline & & -3 & \\
\hline & & 10 & 10700 \\
\hline
\end{tabular}




$\begin{array}{rrrr}\mathrm{H} & 2.120910 & 2.630937 & -0.740153 \\ \mathrm{H} & 3.523582 & 2.138830 & -2.681408 \\ \mathrm{H} & 2.879350 & -0.655884 & 3.022261\end{array}$

Figure S23D Cation C5 TS 11

\begin{tabular}{|c|c|c|c|}
\hline & & & \\
\hline & 5557 & 215 & \\
\hline & -2.997112 & 287 & \\
\hline & -1.115574 & -0.824743 & tus \\
\hline & -0.469252 & 3.864005 & -1.42332 \\
\hline & -2.917877 & -1.845496 & -0.80677 \\
\hline & -4.194963 & -1.789239 & 5879 \\
\hline & -5.293459 & -2.173880 & -0.6522 \\
\hline & -4.851220 & -1.50 & 0.66272 \\
\hline & -1.767698 & -0.9 & \\
\hline$\checkmark$ & -0.314013 & & \\
\hline 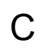 & 0.381383 & & \\
\hline 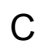 & 0.205039 & & \\
\hline 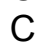 & -0.123085 & & \\
\hline 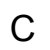 & & & \\
\hline c & & & \\
\hline 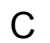 & & & \\
\hline 乙 & & & \\
\hline v & -0.9 & -0.4 & \\
\hline & -3.3 & -1. & \\
\hline & -1.5 & -0.7 & \\
\hline & -0.3 & 1.2 & \\
\hline & -0 . & $-1 . \varepsilon$ & \\
\hline & -0 . & & \\
\hline & -2.5 & $-2 . \varepsilon$ & \\
\hline & -4.1 & -2. & \\
\hline & -4.3 & -0.7 & \\
\hline & -5.3 & -3.2 & \\
\hline & -6.2 & $-1 . \varepsilon$ & -0. \\
\hline & -5.1 & -2. & \\
\hline & -5.2 & -0. & \\
\hline & 0.3 & -0.1 & \\
\hline & -0.9 & & \\
\hline & & -0.3 & -0 \\
\hline 7 & 0.1 & & \\
\hline H & -0.4 & & \\
\hline $\mathrm{H}$ & -0.8 & & \\
\hline S & 80 & -1.5 & \\
\hline C & 3.70 & 485 & $-0 . \varsigma$ \\
\hline C & 306 & -3.7 & \\
\hline 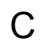 & 279 & -2.6 & \\
\hline C & 4.31 & -2.8 & $-0 . \varepsilon$ \\
\hline C & 3.83 & -0.6 & -0.7 \\
\hline C & 3.3 & -1.2 & \\
\hline C & 2.74 & -0.4 & \\
\hline C & 2.51 & & \\
\hline C & & & \\
\hline & & -1.4 & \\
\hline & 4.0 & 120 & $100 ?$ \\
\hline
\end{tabular}

$\begin{array}{rrrr}\mathrm{H} & 2.322272 & -3.790053 & 1.597413 \\ \mathrm{H} & 3.755477 & -4.686080 & 1.070177 \\ \mathrm{H} & 4.725643 & -3.711899 & -1.281542 \\ \mathrm{H} & 2.435817 & -0.890903 & 2.363296 \\ \mathrm{H} & 2.342284 & 1.570889 & 2.008838 \\ \mathrm{H} & 2.945236 & 2.581638 & -0.188259 \\ \mathrm{H} & 3.884690 & -3.477151 & 2.356679\end{array}$

Figure S23D Cation C5 Product

\begin{tabular}{|c|c|c|c|}
\hline & $-(-c)$ & 0.9 & \\
\hline & -2.710496 & -0.836337 & דרי \\
\hline & -2.995079 & -0.248235 & 596 \\
\hline & -1.264673 & -1.175376 & 05578 \\
\hline & -1.213604 & 3.197802 & -1. \\
\hline & -3.247293 & -1.936327 & \\
\hline & -4.514375 & 633 & -1 \\
\hline & -5.644248 & 54 & \\
\hline & -5.0 & & \\
\hline & -1.9 & 56 & \\
\hline & $-0.1 \varepsilon$ & -0 . & \\
\hline & 0.4 & & \\
\hline & & 93 & \\
\hline & -0.5 & & \\
\hline & -0.2 & & \\
\hline & -0.5 & & \\
\hline & -1 & & \\
\hline & -1. & & \\
\hline & $-1 . C$ & -0 & \\
\hline & -3. & & \\
\hline & -1 & 985 & 045 \\
\hline & -0.6 & & -2 \\
\hline & -1.1 & 722 & 899 \\
\hline & -1.4 & & -2 \\
\hline & -3.0 & $-3 .($ & -0 . \\
\hline & -4.5 & -2 & -2 \\
\hline & -4.4 & 24 & -2 \\
\hline & -5.8 & 504 & 1985 \\
\hline & -6.5 & 141 & 3857 \\
\hline & -5.425435 & 087 & 335 \\
\hline & -5.2 & & \\
\hline & 0.5 & -0 . & 7 \\
\hline & -0.6 & & 208 \\
\hline & 0.9 & $-0 . \varepsilon$ & -1. \\
\hline & -0.2 & 453 & 33 \\
\hline & -1.4 & & 829 \\
\hline & -2.0 & 93 & -0. \\
\hline & & -0.2 & -1.1 \\
\hline & & & -0 \\
\hline & & -3 & \\
\hline & & & \\
\hline & & -1.5 & $-0 . t$ \\
\hline & & & -0.5 \\
\hline & & -0.7 & \\
\hline & 2.800321 & -0.550817 & 1.00207 \\
\hline
\end{tabular}




$\begin{array}{rrrr}\mathrm{C} & 2.044199 & 0.725599 & 0.855390 \\ \mathrm{C} & 2.634162 & 1.697850 & -0.117362 \\ \mathrm{H} & 6.174978 & 0.268690 & -1.767181 \\ \mathrm{H} & 4.199652 & 2.224283 & -1.474893 \\ \mathrm{H} & 3.881363 & -3.707361 & 0.797459 \\ \mathrm{H} & 5.649247 & -3.821165 & 0.817125 \\ \mathrm{H} & 6.756477 & -2.028379 & -0.924340 \\ \mathrm{H} & 2.424275 & -1.300720 & 1.690102 \\ \mathrm{H} & 2.113034 & 1.216668 & 1.846835 \\ \mathrm{H} & 2.098671 & 2.629977 & -0.255181 \\ \mathrm{H} & 4.795755 & -2.985517 & 2.125744\end{array}$

Figure S23D Cation C6 TS 11

$\begin{array}{lrrr}\text { N } & 0.282788 & 0.644691 & -1.446866 \\ \mathrm{C} & -3.035039 & -0.389201 & 1.421128 \\ \mathrm{O} & -3.531108 & 0.407198 & 2.215769 \\ \mathrm{C} & -1.531379 & -0.715268 & 1.398055 \\ \mathrm{C} & -0.269550 & 3.011137 & -2.043863 \\ \mathrm{C} & -3.112645 & -1.999073 & -0.483448 \\ \mathrm{C} & -4.183456 & -2.123295 & -1.577146 \\ \mathrm{C} & -5.499021 & -1.963915 & -0.793860 \\ \mathrm{C} & -5.175577 & -0.904735 & 0.271996 \\ \mathrm{C} & -1.759754 & -1.474374 & -0.968661 \\ \mathrm{C} & -0.636728 & 0.371172 & 2.049927 \\ \mathrm{C} & 0.190495 & 0.889011 & 0.886202 \\ \mathrm{C} & 0.220127 & -0.147416 & -0.237137 \\ \mathrm{C} & -0.018186 & 1.949740 & -1.162944 \\ \mathrm{C} & -0.057927 & 2.147597 & 0.250339 \\ \mathrm{C} & -0.353364 & 3.418641 & 0.782611 \\ \mathrm{C} & -0.596875 & 4.464374 & -0.090186 \\ \mathrm{C} & -0.552690 & 4.252805 & -1.489209 \\ \mathrm{~N} & -1.066862 & -0.816354 & -0.001759 \\ \mathrm{~N} & -3.729250 & -1.076520 & 0.490697 \\ \mathrm{O} & -1.346541 & -1.626115 & -2.116799 \\ \mathrm{H} & 0.063298 & 0.197034 & -2.328912 \\ \mathrm{H} & -1.390797 & -1.677693 & 1.908673 \\ \mathrm{H} & -0.249759 & 2.864305 & -3.118668 \\ \mathrm{H} & -2.936877 & -2.979302 & -0.011989 \\ \mathrm{H} & -4.109473 & -3.071746 & -2.112988 \\ \mathrm{H} & -4.058485 & -1.313680 & -2.303678 \\ \mathrm{H} & -5.768343 & -2.910457 & -0.312289 \\ \mathrm{H} & -6.333961 & -1.662946 & -1.431453 \\ \mathrm{H} & -5.719284 & -1.047853 & 1.209713 \\ \mathrm{H} & -5.373795 & 0.114107 & -0.081442 \\ \mathrm{H} & -0.037598 & -0.066421 & 2.846452 \\ \mathrm{H} & -1.260423 & 1.155482 & 2.483610 \\ \mathrm{H} & 1.042700 & -0.869174 & -0.197649 \\ \mathrm{H} & -0.386353 & 3.569928 & 1.857523 \\ \mathrm{H} & -0.827775 & 5.452743 & 0.292730 \\ \mathrm{H} & -0.754449 & 5.089075 & -2.152370 \\ \mathrm{~N} & 4.420825 & 0.137988 & -1.342505 \\ \mathrm{C} & 2.951644 & 1.204971 & 0.387142 \\ \mathrm{C} & 5.123178 & -3.304831 & -0.128381 \\ \mathrm{C} & 4.678623 & -1.892112 & -0.363693\end{array}$

$\begin{array}{lrrr}\mathrm{C} & 4.999878 & -1.093182 & -1.454821 \\ \mathrm{C} & 3.701266 & 0.187841 & -0.163676 \\ \mathrm{C} & 3.855940 & -1.090782 & 0.472363 \\ \mathrm{C} & 3.234678 & -1.307586 & 1.730636 \\ \mathrm{C} & 2.494841 & -0.296450 & 2.296499 \\ \mathrm{C} & 2.275395 & 0.950977 & 1.616826 \\ \mathrm{H} & 4.509435 & 0.887728 & -2.014137 \\ \mathrm{H} & 2.853375 & 2.168753 & -0.102255 \\ \mathrm{H} & 4.268135 & -3.987492 & -0.054004 \\ \mathrm{H} & 5.760536 & -3.656043 & -0.944905 \\ \mathrm{H} & 5.616076 & -1.332193 & -2.311271 \\ \mathrm{H} & 3.370866 & -2.250598 & 2.251348 \\ \mathrm{H} & 2.063810 & -0.436985 & 3.282400 \\ \mathrm{H} & 1.971117 & 1.806099 & 2.212811 \\ \mathrm{H} & 5.693454 & -3.396254 & 0.803823\end{array}$

Figure S23D Cation C6 Product 11

$\begin{array}{lrrr}\text { N } & 0.168642 & 0.394571 & -1.466474 \\ \mathrm{C} & -3.072888 & -0.431485 & 1.424746 \\ \mathrm{O} & -3.381382 & 0.357176 & 2.316885 \\ \mathrm{C} & -1.668132 & -1.048162 & 1.294805 \\ \mathrm{C} & -0.641526 & 2.772177 & -1.691039 \\ \mathrm{C} & -3.515386 & -1.785976 & -0.618621 \\ \mathrm{C} & -4.618686 & -1.589365 & -1.668184 \\ \mathrm{C} & -5.857771 & -1.263766 & -0.815443 \\ \mathrm{C} & -5.306373 & -0.403603 & 0.333660 \\ \mathrm{C} & -2.101522 & -1.468799 & -1.111697 \\ \mathrm{C} & -0.555606 & -0.271073 & 2.021804 \\ \mathrm{C} & 0.352193 & 0.311056 & 0.910478 \\ \mathrm{C} & 0.094140 & -0.550155 & -0.364885 \\ \mathrm{C} & -0.231841 & 1.646064 & -0.976878 \\ \mathrm{C} & -0.112376 & 1.676774 & 0.422680 \\ \mathrm{C} & -0.388286 & 2.842772 & 1.128523 \\ \mathrm{C} & -0.798885 & 3.981136 & 0.423350 \\ \mathrm{C} & -0.923516 & 3.937355 & -0.969907 \\ \mathrm{~N} & -1.259926 & -1.080984 & -0.119984 \\ \mathrm{~N} & -3.917616 & -0.872197 & 0.469045 \\ \mathrm{O} & -1.773660 & -1.547210 & -2.296960 \\ \mathrm{H} & -0.245371 & 0.055398 & -2.330422 \\ \mathrm{H} & -1.730707 & -2.073238 & 1.686633 \\ \mathrm{H} & -0.742231 & 2.744607 & -2.772042 \\ \mathrm{H} & -3.518144 & -2.826036 & -0.254411 \\ \mathrm{H} & -4.745298 & -2.472641 & -2.297613 \\ \mathrm{H} & -4.356684 & -0.747306 & -2.317050 \\ \mathrm{H} & -6.294573 & -2.186115 & -0.416554 \\ \mathrm{H} & -6.634128 & -0.743444 & -1.382000 \\ \mathrm{H} & -5.844700 & -0.536664 & 1.276164 \\ \mathrm{H} & -5.311186 & 0.665698 & 0.090407 \\ \mathrm{H} & -0.017079 & -0.941926 & 2.691280 \\ \mathrm{H} & -0.997211 & 0.517358 & 2.634675 \\ \mathrm{H} & 0.800398 & -1.372166 & -0.512287 \\ \mathrm{H} & -0.302018 & 2.873679 & 2.211940 \\ \mathrm{H} & -1.028041 & 4.896268 & 0.960380 \\ \mathrm{H} & -1.250461 & 4.823887 & -1.506250 \\ & & & \end{array}$




$\begin{array}{lrrr}\mathrm{N} & 4.779457 & 0.844622 & -1.151920 \\ \mathrm{C} & 2.714170 & 0.992737 & 0.227382 \\ \mathrm{C} & 6.429390 & -2.348788 & -0.210321 \\ \mathrm{C} & 5.543922 & -1.148574 & -0.366203 \\ \mathrm{C} & 5.758958 & -0.070808 & -1.249783 \\ \mathrm{C} & 3.862599 & 0.415356 & -0.193826 \\ \mathrm{C} & 4.352643 & -0.851884 & 0.303651 \\ \mathrm{C} & 3.599154 & -1.529113 & 1.315608 \\ \mathrm{C} & 2.451074 & -0.974347 & 1.775609 \\ \mathrm{C} & 1.902901 & 0.336331 & 1.294319 \\ \mathrm{H} & 4.719801 & 1.701220 & -1.687705 \\ \mathrm{H} & 2.371589 & 1.940826 & -0.173234 \\ \mathrm{H} & 5.915727 & -3.264608 & -0.524454 \\ \mathrm{H} & 7.334612 & -2.248246 & -0.814988 \\ \mathrm{H} & 6.578373 & 0.069944 & -1.943049 \\ \mathrm{H} & 3.956770 & -2.473638 & 1.712877 \\ \mathrm{H} & 1.905312 & -1.482826 & 2.561932 \\ \mathrm{H} & 1.937171 & 1.026408 & 2.159582 \\ \mathrm{H} & 6.734403 & -2.483403 & 0.833155\end{array}$

Figure S23D Cation C7 TS 11

$\begin{array}{lrrr}\text { N } & -0.527346 & 0.146970 & 1.286103 \\ \mathrm{C} & 3.172773 & 0.085885 & -1.319558 \\ \mathrm{O} & 3.454134 & 0.941467 & -2.156795 \\ \mathrm{C} & 1.845911 & -0.692482 & -1.341597 \\ \mathrm{C} & -0.923877 & 2.576526 & 1.723200 \\ \mathrm{C} & 3.611477 & -1.279184 & 0.724723 \\ \mathrm{C} & 4.571094 & -0.960291 & 1.880368 \\ \mathrm{C} & 5.829806 & -0.455489 & 1.153495 \\ \mathrm{C} & 5.279595 & 0.352766 & -0.033354 \\ \mathrm{C} & 2.127851 & -1.198525 & 1.086885 \\ \mathrm{C} & 0.706530 & 0.016135 & -2.119050 \\ \mathrm{C} & -0.350081 & 0.271807 & -1.055321 \\ \mathrm{C} & -0.110613 & -0.644707 & 0.148886 \\ \mathrm{C} & -0.700278 & 1.447219 & 0.915843 \\ \mathrm{C} & -0.605750 & 1.569557 & -0.504236 \\ \mathrm{C} & -0.733025 & 2.834221 & -1.116589 \\ \mathrm{C} & -0.954194 & 3.941951 & -0.317899 \\ \mathrm{C} & -1.046213 & 3.803730 & 1.090295 \\ \mathrm{~N} & 1.338490 & -0.849788 & 0.036835 \\ \mathrm{~N} & 3.977120 & -0.282273 & -0.301119 \\ \mathrm{O} & 1.695424 & -1.419724 & 2.216437 \\ \mathrm{H} & -0.276105 & -0.155293 & 2.219116 \\ \mathrm{H} & 2.045845 & -1.679390 & -1.780700 \\ \mathrm{H} & -0.989035 & 2.487699 & 2.802483 \\ \mathrm{H} & 3.803394 & -2.295378 & 0.344566 \\ \mathrm{H} & 4.753631 & -1.832904 & 2.510634 \\ \mathrm{H} & 4.138383 & -0.173717 & 2.507328 \\ \mathrm{H} & 6.419676 & -1.303320 & 0.788059 \\ \mathrm{H} & 6.474122 & 0.148339 & 1.797224 \\ \mathrm{H} & 5.913058 & 0.307119 & -0.923392 \\ \mathrm{H} & 5.126270 & 1.408887 & 0.217919 \\ \mathrm{H} & 0.355244 & -0.616615 & -2.933232 \\ \mathrm{H} & 1.076584 & 0.948040 & -2.551477\end{array}$

$\begin{array}{rrrr}\mathrm{H} & -0.638048 & -1.604183 & 0.132544 \\ \mathrm{H} & -0.655878 & 2.932906 & -2.195503 \\ \mathrm{H} & -1.050607 & 4.926012 & -0.764193 \\ \mathrm{H} & -1.213348 & 4.691352 & 1.693528 \\ \mathrm{~N} & -3.615715 & 0.934179 & -0.224470 \\ \mathrm{C} & -2.238938 & -0.350496 & -1.901063 \\ \mathrm{C} & -5.024906 & -1.526235 & 2.188425 \\ \mathrm{C} & -4.349514 & -0.751561 & 1.098394 \\ \mathrm{C} & -4.364471 & 0.601002 & 0.908829 \\ \mathrm{C} & -3.116791 & -0.188985 & -0.786158 \\ \mathrm{C} & -3.552848 & -1.291776 & 0.014268 \\ \mathrm{C} & -3.198665 & -2.579233 & -0.372326 \\ \mathrm{C} & -2.431945 & -2.775954 & -1.545785 \\ \mathrm{C} & -2.005459 & -1.703170 & -2.308078 \\ \mathrm{H} & -3.513594 & 1.872212 & -0.588496 \\ \mathrm{H} & -2.167527 & 0.435453 & -2.647088 \\ \mathrm{H} & -4.300645 & -2.095980 & 2.783297 \\ \mathrm{H} & -5.563613 & -0.857947 & 2.866280 \\ \mathrm{H} & -4.854184 & 1.378417 & 1.477599 \\ \mathrm{H} & -3.520538 & -3.438635 & 0.209189 \\ \mathrm{H} & -2.195766 & -3.787703 & -1.859102 \\ \mathrm{H} & -1.450000 & -1.884123 & -3.222397 \\ \mathrm{H} & -5.746441 & -2.245078 & 1.781654\end{array}$

Figure S23D Cation C7 Product

$\begin{array}{lrrr}11 & & & \\ \text { N } & -0.389877 & 0.143650 & 1.390111 \\ \mathrm{C} & 3.050916 & -0.226684 & -1.355094 \\ \mathrm{O} & 3.194813 & 0.508531 & -2.330615 \\ \mathrm{C} & 1.825902 & -1.140236 & -1.163382 \\ \mathrm{C} & -0.245296 & 2.655794 & 1.321071 \\ \mathrm{C} & 3.724612 & -1.198254 & 0.837609 \\ \mathrm{C} & 4.716151 & -0.631846 & 1.864067 \\ \mathrm{C} & 5.873344 & -0.120993 & 0.987129 \\ \mathrm{C} & 5.176221 & 0.450943 & -0.258074 \\ \mathrm{C} & 2.259511 & -1.164795 & 1.279453 \\ \mathrm{C} & 0.591279 & -0.735558 & -1.987940 \\ \mathrm{C} & -0.469930 & -0.264977 & -0.962592 \\ \mathrm{C} & -0.061694 & -0.881347 & 0.414251 \\ \mathrm{C} & -0.312212 & 1.381815 & 0.750978 \\ \mathrm{C} & -0.378341 & 1.215857 & -0.644451 \\ \mathrm{C} & -0.391986 & 2.323415 & -1.489445 \\ \mathrm{C} & -0.335968 & 3.605053 & -0.927128 \\ \mathrm{C} & -0.260425 & 3.759227 & 0.464273 \\ \mathrm{~N} & 1.386110 & -1.101225 & 0.241347 \\ \mathrm{~N} & 3.940743 & -0.344539 & -0.347884 \\ \mathrm{O} & 1.915697 & -1.178240 & 2.462180 \\ \mathrm{H} & 0.062618 & 0.024631 & 2.291979 \\ \mathrm{H} & 2.139437 & -2.160216 & -1.426563 \\ \mathrm{H} & -0.188021 & 2.784162 & 2.397611 \\ \mathrm{H} & 3.979633 & -2.243180 & 0.598366 \\ \mathrm{H} & 5.023488 & -1.384909 & 2.592571 \\ \mathrm{H} & 4.243792 & 0.191911 & 2.408973 \\ \mathrm{H} & 6.526924 & -0.952840 & 0.701774 \\ \mathrm{H} & 6.486921 & 0.627846 & 1.494201\end{array}$




$\begin{array}{rrrr}\mathrm{H} & 5.762485 & 0.343997 & -1.174863 \\ \mathrm{H} & 4.922932 & 1.511468 & -0.141186 \\ \mathrm{H} & 0.247527 & -1.582815 & -2.582876 \\ \mathrm{H} & 0.858476 & 0.063139 & -2.683060 \\ \mathrm{H} & -0.557209 & -1.822894 & 0.664650 \\ \mathrm{H} & -0.436619 & 2.202233 & -2.568963 \\ \mathrm{H} & -0.337830 & 4.478838 & -1.570818 \\ \mathrm{H} & -0.209199 & 4.757879 & 0.888586 \\ \mathrm{~N} & -3.309709 & 1.174502 & -0.279515 \\ \mathrm{C} & -1.964776 & -0.678065 & -1.402894 \\ \mathrm{C} & -6.045628 & -0.332426 & 1.747708 \\ \mathrm{C} & -4.882094 & 0.032276 & 0.879481 \\ \mathrm{C} & -4.416610 & 1.276251 & 0.585506 \\ \mathrm{C} & -3.036144 & -0.100200 & -0.543591 \\ \mathrm{C} & -4.018303 & -0.884573 & 0.153046 \\ \mathrm{C} & -4.022192 & -2.250520 & -0.008487 \\ \mathrm{C} & -3.064556 & -2.885460 & -0.866912 \\ \mathrm{C} & -2.119253 & -2.163820 & -1.522970 \\ \mathrm{H} & -2.782199 & 1.967708 & -0.629816 \\ \mathrm{H} & -2.062438 & -0.257351 & -2.416648 \\ \mathrm{H} & -5.733746 & -0.969782 & 2.583291 \\ \mathrm{H} & -6.515751 & 0.562907 & 2.162822 \\ \mathrm{H} & -4.761147 & 2.250887 & 0.898453 \\ \mathrm{H} & -4.761619 & -2.862981 & 0.500353 \\ \mathrm{H} & -3.112304 & -3.961739 & -0.992615 \\ \mathrm{H} & -1.419111 & -2.680493 & -2.169513 \\ \mathrm{H} & -6.807154 & -0.882706 & 1.182687\end{array}$

Compound 6 Radical Cation

$\begin{array}{lrrr}12 & & & \\ \mathrm{~N} & 3.619127 & 1.673002 & 0.931523 \\ \mathrm{C} & -1.733273 & -1.099358 & 0.570575 \\ \mathrm{O} & -1.580032 & -2.071640 & 1.312202 \\ \mathrm{C} & -0.534835 & -0.204420 & 0.182028 \\ \mathrm{C} & 5.078210 & 0.312828 & -0.611681 \\ \mathrm{C} & -3.137632 & 0.386325 & -0.872211 \\ \mathrm{C} & -4.610126 & 0.742506 & -0.627587 \\ \mathrm{C} & -5.248062 & -0.635664 & -0.374518 \\ \mathrm{C} & -4.176133 & -1.421170 & 0.405296 \\ \mathrm{C} & -2.148826 & 1.522737 & -0.668350 \\ \mathrm{C} & 0.484949 & -0.227703 & 1.351284 \\ \mathrm{C} & 1.834238 & 0.285755 & 0.991920 \\ \mathrm{C} & 2.402535 & 1.516279 & 1.450478 \\ \mathrm{C} & 3.936560 & 0.574921 & 0.111790 \\ \mathrm{C} & 2.821250 & -0.304333 & 0.147473 \\ \mathrm{C} & 2.878742 & -1.511649 & -0.579267 \\ \mathrm{C} & 4.030813 & -1.792945 & -1.321724 \\ \mathrm{C} & 5.106054 & -0.901486 & -1.340272 \\ \mathrm{~N} & -0.957245 & 1.159996 & -0.111026 \\ \mathrm{~N} & -2.916782 & -0.741228 & 0.045410 \\ \mathrm{O} & -2.399530 & 2.672308 & -1.009656 \\ \mathrm{H} & 4.226815 & 2.465868 & 1.109906 \\ \mathrm{H} & -0.061681 & -0.659382 & -0.701903 \\ \mathrm{H} & 5.922524 & 0.993590 & -0.629283 \\ \mathrm{H} & -3.011950 & 0.045210 & -1.912663\end{array}$

$\begin{array}{rrrr}\mathrm{H} & -5.047387 & 1.275625 & -1.473732 \\ \mathrm{H} & -4.690320 & 1.381850 & 0.258280 \\ \mathrm{H} & -5.458007 & -1.132239 & -1.328077 \\ \mathrm{H} & -6.187255 & -0.569236 & 0.179932 \\ \mathrm{H} & -4.131840 & -2.479203 & 0.131553 \\ \mathrm{H} & -4.313902 & -1.360715 & 1.490264 \\ \mathrm{H} & 0.548575 & -1.276145 & 1.664838 \\ \mathrm{H} & 0.070359 & 0.339949 & 2.189549 \\ \mathrm{H} & 1.970350 & 2.249518 & 2.118082 \\ \mathrm{H} & 2.052844 & -2.215238 & -0.559595 \\ \mathrm{H} & 4.091014 & -2.716637 & -1.886685 \\ \mathrm{H} & 5.990802 & -1.139813 & -1.921274 \\ \mathrm{H} & -0.256931 & 1.893523 & -0.102324\end{array}$

Iron Oxo Anion Triplet

$-13$

$\begin{array}{lrrr}\text { Fe } & -0.073428 & -0.001837 & -0.310750 \\ \mathrm{~N} & -1.285124 & 1.621577 & -0.180319 \\ \mathrm{~N} & 1.550558 & 1.202948 & -0.287649 \\ \mathrm{~N} & -1.696456 & -1.204347 & -0.144644 \\ \mathrm{~N} & 1.140382 & -1.622091 & -0.276528 \\ \mathrm{C} & -2.653288 & 1.625993 & -0.140660 \\ \mathrm{C} & 2.861008 & 0.816128 & -0.389064 \\ \mathrm{C} & -0.893012 & 2.933033 & -0.183909 \\ \mathrm{C} & 1.550140 & 2.572226 & -0.278258 \\ \mathrm{C} & -3.008853 & -0.817351 & -0.108820 \\ \mathrm{C} & 2.505740 & -1.627396 & -0.376674 \\ \mathrm{C} & -1.694549 & -2.574003 & -0.127759 \\ \mathrm{C} & 0.748032 & -2.933298 & -0.248929 \\ \mathrm{C} & -3.145308 & 2.987235 & -0.115666 \\ \mathrm{C} & 3.719618 & 1.979164 & -0.432436 \\ \mathrm{C} & -2.052645 & 3.798599 & -0.140802 \\ \mathrm{C} & 2.906279 & 3.069250 & -0.359767 \\ \mathrm{C} & -3.868012 & -1.980310 & -0.060118 \\ \mathrm{C} & 2.996351 & -2.988679 & -0.404731 \\ \mathrm{C} & -3.052198 & -3.070631 & -0.070422 \\ \mathrm{C} & 1.905274 & -3.799150 & -0.322812 \\ \mathrm{H} & -4.192029 & 3.263174 & -0.079544 \\ \mathrm{H} & 4.799621 & 1.945343 & -0.506726 \\ \mathrm{H} & -2.013474 & 4.880896 & -0.131048 \\ \mathrm{H} & 3.178594 & 4.117521 & -0.364451 \\ \mathrm{H} & -4.949813 & -1.945731 & -0.021225 \\ \mathrm{H} & 4.040958 & -3.265924 & -0.475557 \\ \mathrm{H} & -3.323941 & -4.118684 & -0.043185 \\ \mathrm{H} & 1.866285 & -4.881470 & -0.313659 \\ \mathrm{C} & -3.464513 & 0.497224 & -0.110210 \\ \mathrm{C} & 3.314956 & -0.497415 & -0.436220 \\ \mathrm{C} & -0.565377 & -3.384350 & -0.172930 \\ \mathrm{C} & 0.421338 & 3.383954 & -0.223028 \\ \mathrm{H} & -4.538521 & 0.653658 & -0.075410 \\ \mathrm{H} & 4.386730 & -0.653329 & -0.515941 \\ \mathrm{H} & -0.722758 & -4.458756 & -0.156214 \\ \mathrm{H} & 0.580286 & 4.458240 & -0.221891 \\ \mathrm{O} & -0.140198 & -0.004882 & -1.957473 \\ \mathrm{~S} & -0.077129 & -0.034934 & 2.209741\end{array}$


$\begin{array}{llll}C & 1.657207 & 0.073177 & 2.813500\end{array}$

H $2.134832 \quad 1.0079892 .502419$
$\begin{array}{llll}H & 1.660012 & 0.034311 & 3.908788\end{array}$

$\begin{array}{llll}H & 2.263352 & -0.758495 & 2.439339\end{array}$ 
Supplementary Figure 23. Reaction coordinate diagrams for formation of (+)-naseseazine B (2), and single electron oxidation to radical cation.

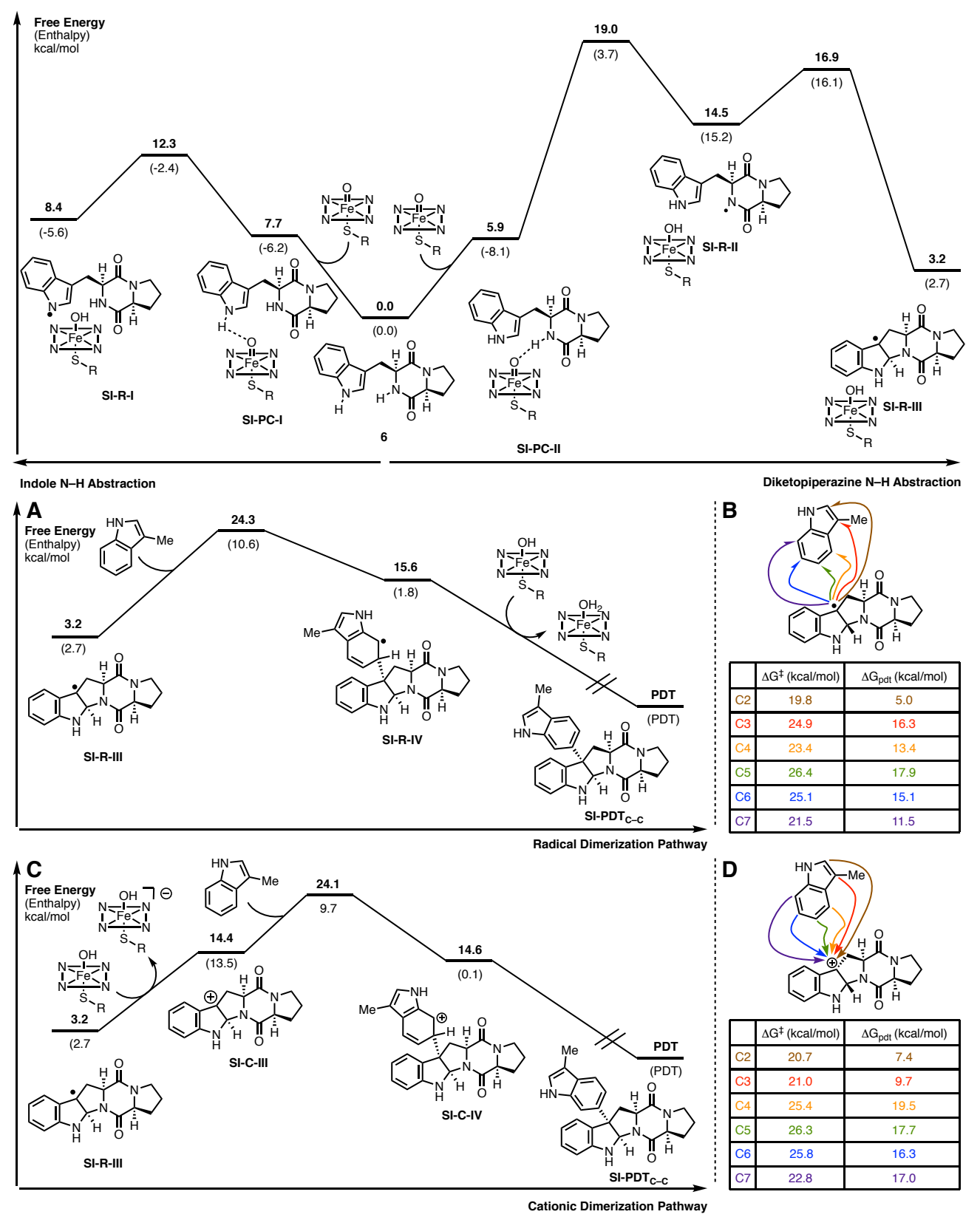

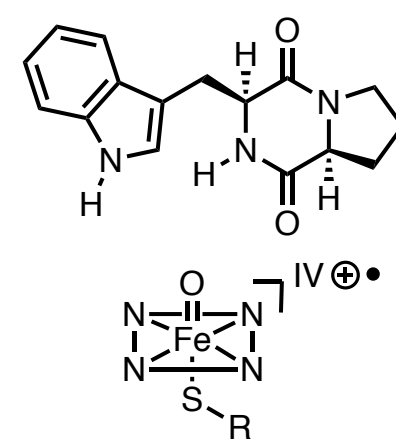

0.0

$(0.0)$<smiles>CC(C)C[C@H]1NC(=O)[C@@H]2CCCN2C1=O</smiles>

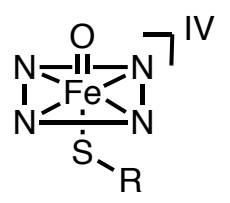

41.2

(41.3) 


\section{Computational References:}

6. Schrödinger Release 2017-2: MacroModel, Schrödinger, LLC, New York, NY, 2017.

7. Gaussian 09, Revision D.01, Frisch, M. J.; Trucks, G. W.; Schlegel, H. B.; Scuseria, G. E.; Robb, M. A.; Cheeseman, J. R.; Scalmani, G.; Barone, V.; Mennucci, B.; Petersson, G. A.; Nakatsuji, H.; Caricato, M.; Li, X.; Hratchian, H. P.; Izmaylov, A. F.; Bloino, J.; Zheng, G.; Sonnenberg, J. L.; Hada, M.; Ehara, M.; Toyota, K.; Fukuda, R.; Hasegawa, J.; Ishida, M.; Nakajima, T.; Honda, Y.; Kitao, O.; Nakai, H.; Vreven, T.; Montgomery, Jr., J. A.; Peralta, J. E.; Ogliaro, F.; Bearpark, M.; Heyd, J. J.; Brothers, E.; Kudin, K. N.; Staroverov, V. N.; Keith, T.; Kobayashi, R.; Normand, J.; Raghavachari, K.; Rendell, A.; Burant, J. C.; lyengar, S. S.; Tomasi, J.; Cossi, M.; Rega, N.; Millam, J. M.; Klene, M.; Knox, J. E.; Cross, J. B.; Bakken, V.; Adamo, C.; Jaramillo, J.; Gomperts, R.; Stratmann, R. E.; Yazyev, O.; Austin, A. J.; Cammi, R.; Pomelli, C.; Ochterski, J. W.; Martin, R. L.; Morokuma, K.; Zakrzewski, V. G.; Voth, G. A.; Salvador, P.; Dannenberg, J. J.; Dapprich, S.; Daniels, A. D.; Farkas, O.; Foresman, J. B.; Ortiz, J. V.; Cioslowski, J.; Fox, D. J. Gaussian, Inc., Wallingford, CT, 2013.

8. Becke, A. D. Density-functional Thermochemistry. III. The Role of Exact Exchange. J. Chem. Phys. 1993, 98, 5648-5652.

9. Lee, C.; Yang, W.; Parr, R. G. Development of the Colle-Salvetti Correlation-Energy Formula into a Functional of the Electron Density. Phys. Rev. B, 1988, 37, 785-789.

10. Hay, P. J.; Wadt, W. R. Ab Initio Effective Core Potentials for Molecular Calculations. Potentials for the Transition Metal Atoms Sc to Hg. J. Chem. Phys. 1985, 82, 270-283.

11. Miertuš, S.; Scrocco, E.; Tomasi, J. Electrostatic Interaction of a Solute with a Continuum. A Direct Utilizaion of Ab Initio Molecular Potentials for the Prevision of Solvent Effects. Chem. Phys. 1981, 55, 117-129.

12. Miertus̃, S.; Tomasi, J. Approximate Evaluations of the Electrostatic Free Energy and Internal Energy Changes in Solution Processes. Chem. Phys. 1982, 65, 239-245.

13. Pascual-ahuir, J. L.; Silla, E.; Tuñon, I. GEPOL: An Improved Description of Molecular Surfaces. III. A New Algorithm for the Computation of a Solvent-Excluding Surface. J. Comput. Chem. 1994, 15, 11271138.

14. Ribeiro, R. F.; Marenich, A. V.; Cramer, C. J.; Truhlar, D. G. Use of Solution-Phase Vibrational Frequencies in Continuum Models for the Free Energy of Solvation. J. Phys. Chem. B 2011, 115, $14556-14562$.

15. Grimme, S.; Antony, J.; Ehrlich, S.; Krieg, H. A Consistent and Accurate Ab Initio Parametrization of Density Functional Dispersion Correction (DFT-D) for the 94 Elements H-Pu. J. Chem. Phys. 2010, 132, 154104.

16. Grimme, S.; Ehrlich, S.; Goerigk, L. Effect of the Damping Function in Dispersion Corrected Density Functional Theory. J. Comput. Chem. 2011, 32, 1456-1465. 


\section{Chemistry}

\section{General Procedures:}

All reactions were performed in oven-dried or flame-dried round-bottom flasks. The flasks were fitted with rubber septa and reactions were conducted under a positive pressure of argon. Gas-tight syringes with stainless steel needles or cannulae were used to transfer air- and moisture-sensitive liquids. Where necessary (so noted), reactions were performed in Schlenk tubes fitted with a PTFE stopcock. Flash column chromatography was performed as described by Still et al. using granular silica gel (60 A pore size, 40-63 $\mu \mathrm{m}$, 4-6\% H2O content, Zeochem). Analytical thin layer chromatography (TLC) was performed using glass plates pre-coated with $0.25 \mathrm{~mm} \mathrm{230-400} \mathrm{mesh} \mathrm{silica} \mathrm{gel} \mathrm{impregnated} \mathrm{with} \mathrm{a} \mathrm{fluorescent} \mathrm{indicator} \mathrm{(254} \mathrm{nm}$ ). TLC plates were visualized by exposure to short wave ultraviolet light $(254 \mathrm{~nm})$ and an aqueous solution of ceric ammonium molybdate (CAM) followed by heating on a hot plate ( 250 $\left.{ }^{\circ} \mathrm{C}\right)$. Organic solutions were concentrated at $29-30{ }^{\circ} \mathrm{C}$ on rotary evaporators capable of achieving a minimum pressure of $\sim 2$ torr.

\section{Materials:}

Commercial reagents and solvents were used as received with the following exceptions: dichloromethane, acetonitrile, tetrahydrofuran, methanol, pyridine, toluene, and triethylamine were purchased from J.T. Baker (CycletainerTM) and were purified by the method of Grubbs et al. under positive argon pressure. Nitroethane was distilled over calcium hydride and stored over $4 \AA$ molecular sieves. Silver hexafluoroantimonate was purchased from Strem Chemicals, Inc. All other solvents and chemicals were purchased from Sigma-Aldrich.

\section{Instrumentation:}

Proton nuclear magnetic resonance (1H NMR) spectra were recorded with Varian inverse probe INOVA-500 and Varian INOVA-500 spectrometers, or a Bruker AVANCE III 400 spectrometer. Chemical shifts are reported in parts per million on the $\delta$ scale, and are referenced from the residual protium in the NMR solvent (CHCl3: $\delta$ 7.26, CD3SOCD2H: $\delta 2.50, \mathrm{CD} 2 \mathrm{HOD}: \delta 3.31)$. Data are reported as follows: chemical shift [multiplicity (s = singlet, $d=$ doublet, $t=$ triplet, $m=$ multiplet), coupling constant(s) in Hertz, integration, assignment]. Carbon13 nuclear magnetic resonance (13C NMR) spectra were recorded with a Varian INOVA-500 spectrometer or a Bruker AVANCE III 400 spectrometer and are reported in parts per million on the $\delta$ scale, and are referenced from the carbon resonances of the solvent (CDCl3: $\delta$ 77.16, DMSO-d6: $\delta$ 39.52, CD3OD: $\delta$ 49.00). Data are reported as follows: chemical shift (assignment). Data are reported as follows: chemical shift (assignment). Infrared data (IR) were obtained with a Perkin-Elmer 2000 FTIR, and are reported as follows: frequency of absorption (cm-1), intensity of absorption ( $\mathrm{s}=$ strong, $\mathrm{m}=$ medium, $\mathrm{w}=$ weak, $\mathrm{br}=$ broad). Optical rotations were measured on a Jasco-1010 polarimeter. UV-Vis spectrophotometric data were acquired on a Varian Cary 50 Bio UV-Vis spectrophotometer. Preprative HPLC was performed on a Waters system with the 1525 Binary HPLC Pump, 2489 UV/Vis Detector, 3100 Mass Detector, System Fluidics Organizer, and 2767 Sample Manager components. We thank Liam P. Kelly for obtaining the mass spectrometric data at the Department of Chemistry's Instrumentation Facility, Massachusetts Institute of Technology. High resolution mass spectra (HRMS) were recorded on a Bruker Daltonics APEXIV 4.7 Tesla FT-ICR-MS using either an electrospray (ESI) or direct analysis in real time (DART) ionization source. 


\section{Positional Numbering System.}

In assigning the ${ }^{1} \mathrm{H}$ and ${ }^{13} \mathrm{C}$ NMR data of all intermediates en route to our total syntheses of (-)-naseseazine $\mathrm{C}$ and (-)-iso-naseseazine $C$, we wished to employ a uniform numbering scheme. This numbering system is also consistent with that employed in the isolation papers of naseseazine $\mathrm{C}$ and iso-naseseazine $\mathrm{B} .^{1,2}$

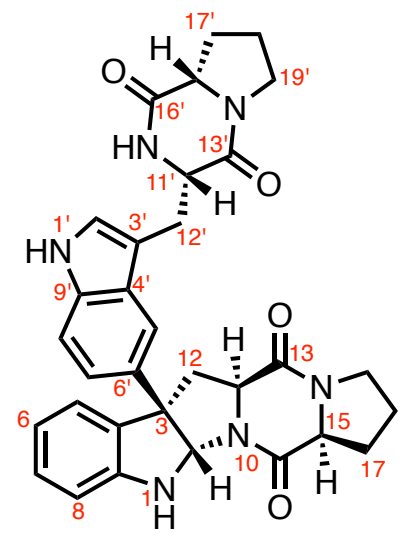

(-)-naseseazine C (4)

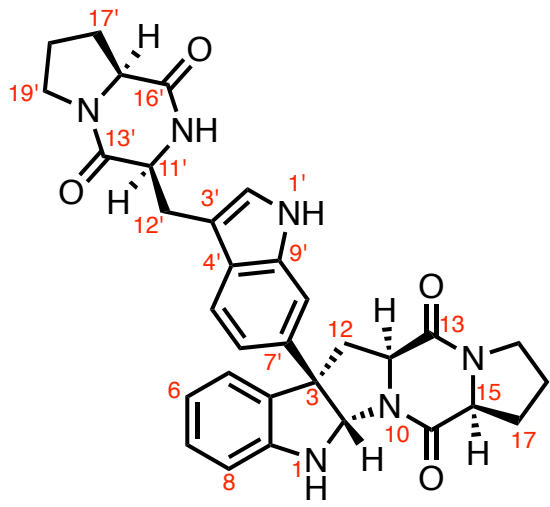

(-)-iso-naseseazine C (11) 


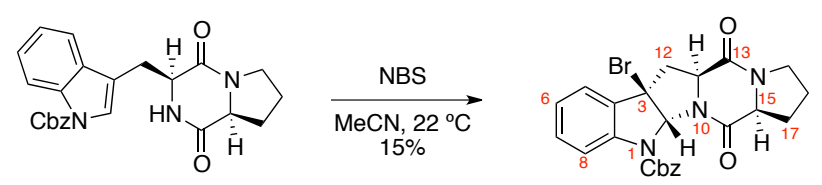

Diketopiperazine bromide (-)-8: A sample of $\mathrm{N}$-bromosuccinimide (641 mg, $3.60 \mathrm{mmol}, 3.00$ equiv) was added in one portion to a stirred suspension of diketopiperazine 7 (500 mg, $1.20 \mathrm{mmol}, 1$ equiv) in acetonitrile $(60 \mathrm{~mL})$. After $25 \mathrm{~min}$, a solution of saturated aqueous sodium thiosulfate solution $(30 \mathrm{~mL})$ was added to the reaction mixture. After $10 \mathrm{~min}$, the reaction mixture was diluted with ethyl acetate $(30 \mathrm{~mL})$, and washed with saturated aqueous sodium thiosulfate solution $(30 \mathrm{~mL})$. The aqueous layer was extracted with ethyl acetate $(3$ $\times 20 \mathrm{~mL}$ ) and the combined organic layers were dried over anhydrous sodium sulfate, were filtered, and were concentrated under reduced pressure. The crude reaction mixture was purified by flash column chromatography on silica gel (eluent: $30 \rightarrow 35 \%$ ethyl acetate in hexanes), including a second chromatographic purification (eluent: $30 \rightarrow 35 \%$ ethyl acetate in hexanes) to afford diketopiperazine bromide (-)-8 (90.5 mg, $14.8 \%)$ as an off-white foam.

Structural assignments were made using additional information from gCOSY, HSQC, HMBC and nOe experiments. $1 \mathrm{H}$ NMR (MHz, CDCl3, $20^{\circ} \mathrm{C}$ ): $\delta 7.69$ (app-br-s, 1H, C8H), 7.48 (app-d, J = 7.3 Hz, 2H, PhCbzo-H), 7.43 (d, J = 7.7 Hz, 1H, C5H), 7.41-7.30 (m, 4H, C7H, PhCbz-m-H, PhCbz-p-H), 7.16 (app-t, J = 7.5 Hz, $1 \mathrm{H}, \mathrm{C} 6 \mathrm{H}), 6.57(\mathrm{~s}, 1 \mathrm{H}, \mathrm{C} 2 \mathrm{H}), 5.43(\mathrm{~d}, \mathrm{~J}=12.2 \mathrm{~Hz}, 1 \mathrm{H}, \mathrm{PhCbzCHa}), 5.31$ (d, J = $12.3 \mathrm{~Hz}, 1 \mathrm{H}, \mathrm{PhCbzCHb}), 4.04$ (app-t, J = 8.2 Hz, 1H, C15H), $3.97(\mathrm{dd}, \mathrm{J}=5.9,11.4 \mathrm{~Hz}, 1 \mathrm{H}, \mathrm{C} 11 \mathrm{H}), 3.62-3.45(\mathrm{~m}, 2 \mathrm{H}, \mathrm{C} 19 \mathrm{H}), 3.35$ (dd, J = 5.9, $13.2 \mathrm{~Hz}, 1 \mathrm{H}, \mathrm{C} 12 \mathrm{Ha}), 3.01(\mathrm{dd}, \mathrm{J}=11.0,13.2 \mathrm{~Hz}, 1 \mathrm{H}, \mathrm{C} 12 \mathrm{Hb}), 2.35-2.23(\mathrm{~m}, 1 \mathrm{H}, \mathrm{C} 17 \mathrm{Ha}), 2.25-2.14(\mathrm{~m}$, 1H, C17Hb), 2.10-1.97 (m, 1H, C18Ha), 1.95-1.80 (m, 1H, C18Hb). 13C NMR (100 MHz, CDCl3, $\left.20^{\circ} \mathrm{C}\right): \delta$ 165.8 (C16), 164.2 (C13), 153.0 (NC=OCbz), 141.4 (C9), 135.8 (PhCbz-i-C), 132.0 (C4), 131.2 (PhCbz), 128.7 (C7), 128.5 (PhCbz-o-C), 128.4 (PhCbz), 125.1 (C6), 123.9 (C5), 117.7 (C8), 83.3 (C2), 68.6, 60.4 (2C, C11, C15), 59.0 (C3), 45.6 (C19), 43.1 (C12), 27.8 (C17), 23.1 (C18). FTIR (thin film) cm-1: 2957 (w), 2887 (w), 1716 (s), 1674 (s), 1329 (m). HRMS (ESI) (m/z):calc'd for C24H23BrN3O4 [M+H]+: 496.0866, found: 496.0858. [a]D24: -168 (c = 0.46, $\mathrm{CH} 2 \mathrm{Cl} 2)$. TLC (70\% ethyl acetate in hexanes), Rf: 0.22 (UV, CAM). 

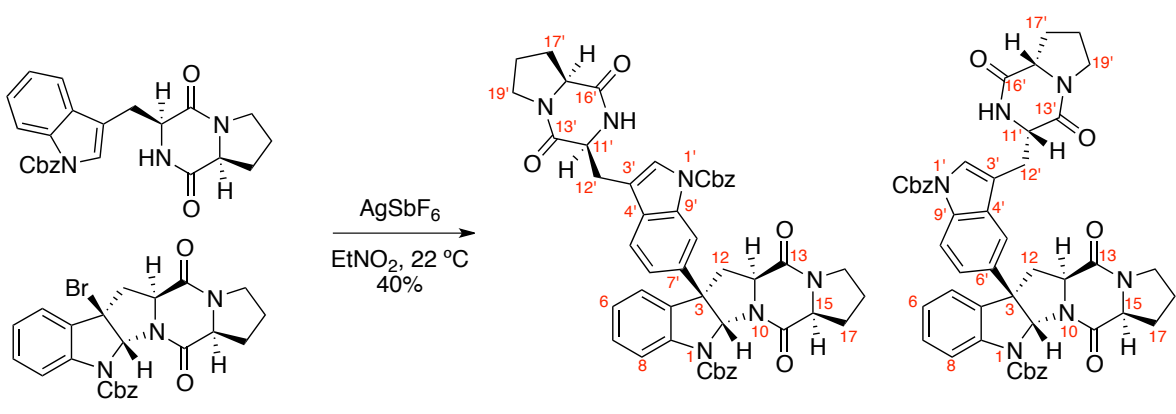

$(-)$-meta:(-)-para $=1.4: 1$

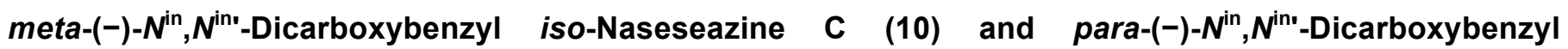
Naseseazine C (9): Diketopiperazine bromide (-)-8 (100 mg, $0.196 \mathrm{mmol}, 1$ equiv) and proline diketopiperazine 7 (245 mg, $0.588 \mathrm{mmol}, 3.00$ equiv) were azeotropically dried from anhydrous benzene $(3 \times$ $0.5 \mathrm{~mL})$ and the residue was suspended in freshly distilled nitroethane $(9.8 \mathrm{~mL})$. Silver hexafluoroantimonate (202 mg, $0.588 \mathrm{mmol}, 3.00$ equiv) was then added as a solid in a single portion. After $4.5 \mathrm{~h}$, the reaction mixture was diluted with dichloromethane $(15 \mathrm{~mL})$ and washed with a mixture of saturated aqueous sodium bicarbonate and saturated aqueous sodium thiosulfate $(10: 1 \mathrm{v} / \mathrm{v}, 15 \mathrm{~mL})$. The resulting aqueous layer was extracted with dichloromethane $(3 \times 10 \mathrm{~mL})$ and the combined organic layers were dried over anhydrous sodium sulfate, were filtered, and were concentrated under reduced pressure. The crude reaction mixture was purified by flash column chromatography on silica gel (eluent: $10 \rightarrow 50 \%$ acetone in dichloromethane) to afford a regioisomeric mixture of (-)-10 and (-)-9 (65.6 mg, 40.1\%, (-)-10:(-)-9, 1.4:1) as a white foam. Regioisomers (-)-10 and (-)-9 were separated for the purpose of full and independent characterization by preparative HPLC [Luna preparative HPLC column, C18, $5 \mu \mathrm{m}, 21.2 \times 250 \mathrm{~mm} ; 7.0 \mathrm{~mL} / \mathrm{min}$; gradient, $5 \% \rightarrow 100 \%$ acetonitrile in water with $0.1 \%$ formic acid, $60 \mathrm{~min} ; t_{R}((-)-10)=48.3 \mathrm{~min}, t_{R}((-)-9)=50.4 \mathrm{~min}$. Structural assignments were made using additional information from gCOSY, HSQC, HMBC and nOe experiments.

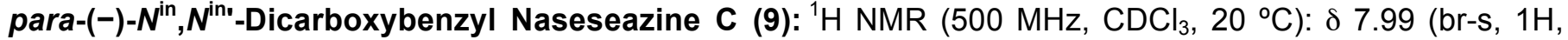
$\left.\mathrm{C}_{8} \mathrm{H}\right), 7.71\left(\mathrm{br}-\mathrm{s}, 1 \mathrm{H}, \mathrm{C}_{8} \mathrm{H}\right), 7.53\left(\mathrm{~s}, 1 \mathrm{H}, \mathrm{C}_{2} \cdot \mathrm{H}\right), 7.48-7.43\left(\mathrm{~m}, 3 \mathrm{H}, \mathrm{C}_{5} \mathrm{H}, \mathrm{Ph}_{\mathrm{Cbz}}-\mathrm{H}\right), 7.43-7.35\left(\mathrm{~m}, 5 \mathrm{H}, \mathrm{Ph}_{\mathrm{Cbz}}-\mathrm{H}\right)$, 7.34-7.26 (m, 4H, $\left.\left.\mathrm{C}_{7} \mathrm{H}\right) \mathrm{Ph}_{\mathrm{Cbz}}-\mathrm{H}\right), 7.22\left(\mathrm{~d}, J=7.4 \mathrm{~Hz}, 1 \mathrm{H}, \mathrm{C}_{5} \mathrm{H}\right), 7.10\left(\right.$ app-t, $\left.J=7.5 \mathrm{~Hz}, 1 \mathrm{H}, \mathrm{C}_{6} \mathrm{H}\right), 7.04(\mathrm{~d}, J=$ $\left.8.8 \mathrm{~Hz}, 1 \mathrm{H}, \mathrm{C}_{7} \mathrm{H}\right), 6.61\left(\mathrm{~s}, 1 \mathrm{H}, \mathrm{C}_{2} \mathrm{H}\right), 5.69\left(\mathrm{~s}, 1 \mathrm{H}, \mathrm{N}_{10} \mathrm{H}\right), 5.42\left(\mathrm{app}-\mathrm{s}, 2 \mathrm{H}, \mathrm{Ph}_{\mathrm{Cbz}} \mathrm{CH}_{2}\right), 5.39(\mathrm{~d}, J=12.2 \mathrm{~Hz}, 1 \mathrm{H}$, $\left.\mathrm{Ph}_{\mathrm{Cbz}^{\prime}} \mathrm{CH}_{\mathrm{a}}\right), 5.28\left(\mathrm{~d}, \mathrm{~J}=12.3 \mathrm{~Hz}, 1 \mathrm{H}, \mathrm{Ph}_{\mathrm{Cbz}^{\prime}} \mathrm{CH}_{\mathrm{b}}\right), 4.31$ (dd, J = 3.5, $\left.10.7 \mathrm{~Hz}, 1 \mathrm{H}, \mathrm{C}_{11} \mathrm{H}\right), 4.15(\mathrm{dd}, J=5.8,11.3$ $\left.\mathrm{Hz}, 1 \mathrm{H}, \mathrm{C}_{11} \mathbf{H}\right), 4.10\left(\mathrm{app}-\mathrm{q}, \mathrm{J}=7.8 \mathrm{~Hz}, 2 \mathrm{H}, \mathrm{C}_{15} \mathbf{H}, \mathrm{C}_{15} \mathbf{H}\right), 3.69-3.47\left(\mathrm{~m}, 5 \mathrm{H}, \mathrm{C}_{12} \mathbf{H}_{\mathrm{a}}, \mathrm{C}_{19} \mathrm{H}, \mathrm{C}_{19} \mathbf{H}\right), 3.16(\mathrm{dd}, \mathrm{J}=$ 5.8, $\left.12.9 \mathrm{~Hz}, 1 \mathrm{H}, \mathrm{C}_{12} \mathrm{H}_{\mathrm{a}}\right), 2.91-2.82\left(\mathrm{~m}, 2 \mathrm{H}, \mathrm{C}_{12} \mathrm{H}_{\mathrm{b}}, \mathrm{C}_{12} \mathrm{H}_{\mathrm{b}}\right), 2.36-2.27\left(\mathrm{~m}, 2 \mathrm{H}, \mathrm{C}_{17} \mathrm{H}\right), 2.27-2.11\left(\mathrm{~m}, 1 \mathrm{H}, \mathrm{C}_{17} \mathrm{H}_{\mathrm{a}}\right)$, 2.11-1.95 (m, 3H, $\left.\mathrm{C}_{17} \mathrm{H}_{\mathrm{b}}, \mathrm{C}_{18} \mathrm{H}\right), 1.96-1.84\left(\mathrm{~m}, 2 \mathrm{H}, \mathrm{C}_{18} \mathrm{H}\right) .{ }^{13} \mathrm{C}$ NMR $\left(125 \mathrm{MHz}, \mathrm{CDCl}_{3}, 20{ }^{\circ} \mathrm{C}\right): \delta 169.5\left(\mathbf{C}_{16^{\prime}}\right)$, $166.0\left(\mathbf{C}_{16}\right), 165.4\left(\mathbf{C}_{13}\right), 164.9\left(\mathbf{C}_{13^{\prime}}\right), 153.1\left(\mathbf{C}_{\mathrm{Cbz}}=\mathrm{O}\right), 150.4\left(\mathbf{C}_{\mathrm{Cbz}^{\prime}}=\mathrm{O}\right), 141.5\left(\mathbf{C}_{9}\right), 136.6\left(\mathbf{C}_{6^{\prime}}\right), 136.1\left(\mathrm{Ph}_{\mathrm{Cbz}}-i-\mathbf{C}\right)$, $135.1\left(\mathbf{C}_{4}\right), 134.8\left(2 \mathrm{C}, \mathbf{C}_{9^{\prime}}, \mathrm{Ph}_{\mathrm{Cbz}^{\prime}-}-\mathbf{C}\right)$ ), $130.0\left(\mathbf{C}_{4^{\prime}}\right), 129.3\left(\mathbf{C}_{7}\right), 129.0\left(2 \mathrm{C}, \mathrm{Ph}_{\mathrm{Cbz}}-\mathbf{C}\right), 128.8\left(\mathrm{Ph}_{\mathrm{Cbz}}-\mathbf{C}\right), 128.6$ $\left(\mathrm{Ph}_{\mathrm{Cbz}}-\mathbf{C}\right), 128.3\left(2 \mathrm{C}, \mathrm{Ph}_{\mathrm{Cbz}}-\mathbf{C}\right), 125.0\left(\mathbf{C}_{2^{\prime}}\right), 124.8\left(\mathbf{C}_{6}\right), 124.0\left(\mathbf{C}_{5}\right), 123.6\left(\mathbf{C}_{7^{\prime}}\right), 117.6\left(\mathbf{C}_{8}\right), 116.3\left(\mathbf{C}_{8^{\prime}}\right), 116.0$ $\left(\mathbf{C}_{3^{\prime}}\right), 115.4\left(\mathbf{C}_{5^{\prime}}\right), 81.9\left(\mathbf{C}_{2}\right), 69.2\left(\mathrm{Ph}_{\mathrm{bbz}^{\prime}}-\mathbf{C H}_{2}\right), 68.3\left(\mathrm{Ph}_{\mathrm{Cbz}^{-}}-\mathbf{C H}_{2}\right), 60.7\left(\mathbf{C}_{11}\right.$ or $\left.\mathbf{C}_{15^{\prime}}\right), 60.6\left(\mathbf{C}_{11}\right.$ or $\left.\mathbf{C}_{15^{\prime}}\right), 59.3\left(\mathbf{C}_{15}\right)$, $58.2\left(\mathbf{C}_{3}\right), 53.9\left(\mathbf{C}_{11^{\prime}}\right), 45.6\left(\mathbf{C}_{19}\right.$ or $\left.\mathbf{C}_{19^{\prime}}\right), 45.5\left(\mathbf{C}_{19}\right.$ or $\left.\mathbf{C}_{19^{\prime}}\right), 39.6\left(\mathbf{C}_{12}\right), 28.4\left(\mathbf{C}_{17^{\prime}}\right), 27.9\left(\mathbf{C}_{17}\right), 26.5\left(\mathbf{C}_{12^{\prime}}\right), 23.1$ $\left(\mathbf{C}_{18^{\prime}}\right), 22.8\left(\mathbf{C}_{18}\right)$. FTIR (thin film) $\mathrm{cm}^{-1}: 3472(\mathrm{br}-\mathrm{m}), 2956(\mathrm{w}), 1716(\mathrm{~s}), 1668(\mathrm{~s}), 1479(\mathrm{~m}), 1401$ (m). HRMS (ESI) $(\mathrm{m} / \mathrm{z})$ : calc'd for $\mathrm{C}_{48} \mathrm{H}_{45} \mathrm{~N}_{6} \mathrm{O}_{8}[\mathrm{M}+\mathrm{H}]^{+}:$833.3293, found: 833.3286. $[\alpha]_{D}{ }^{24}$ : $-128\left(c=0.24, \mathrm{CH}_{2} \mathrm{Cl}_{2}\right)$. TLC (70\% acetone in hexanes), Rf: 0.24 (UV, CAM).

meta-(-)- $\boldsymbol{N}^{\text {in }}, \boldsymbol{N}^{\mathrm{in}}$-Dicarboxybenzyl iso-Naseseazine $\mathbf{C}(10):{ }^{1} \mathrm{H}$ NMR $\left(\mathrm{MHz}, \mathrm{CDCl}_{3}, 20{ }^{\circ} \mathrm{C}\right): \delta 8.19(\mathrm{br}-\mathrm{s}, 1 \mathrm{H}$, $\left.\mathrm{C}_{8} \cdot \mathbf{H}\right), 7.73\left(\mathrm{br}-\mathrm{s}, 1 \mathrm{H}, \mathrm{C}_{8} \mathrm{H}\right), 7.50\left(\mathrm{~s}, 1 \mathrm{H}, \mathrm{C}_{2} \mathbf{H}\right), 7.48-7.38\left(\mathrm{~m}, 6 \mathrm{H}, \mathrm{Ph}_{\mathrm{cbz}}-\mathrm{H}\right), 7.38-7.34\left(\mathrm{~m}, 2 \mathrm{H}, \mathrm{C}_{5} \mathrm{H}, \mathrm{Ph}_{\mathrm{cbz}}-\mathrm{H}\right)$, 7.34-7.26 (m, 4H, C 7 H, $\left.\mathrm{Ph}_{\mathrm{Cbz}}-\mathrm{H}\right), 7.19\left(\mathrm{~d}, J=7.5 \mathrm{~Hz}, 1 \mathrm{H}, \mathrm{C}_{5} \mathrm{H}\right), 7.08\left(\mathrm{~d}, J=7.5 \mathrm{~Hz}, 1 \mathrm{H}, \mathrm{C}_{6} \mathrm{H}\right), 7.07-7.02(\mathrm{~m}$, $\left.1 \mathrm{H}, \mathrm{C}_{6} \mathrm{H}\right), 6.64\left(\mathrm{~s}, 1 \mathrm{H}, \mathrm{C}_{2} \mathrm{H}\right), 5.57\left(\mathrm{~s}, 1 \mathrm{H}, \mathrm{N}_{10} \mathrm{H}\right), 5.41\left(\mathrm{~d}, J=12.3 \mathrm{~Hz}, 1 \mathrm{H}, \mathrm{Ph}_{\mathrm{Cbz}} \mathrm{CH}_{\mathrm{a}}\right), 5.39(\operatorname{app}-\mathrm{s}, 2 \mathrm{H}$, $\left.\mathrm{Ph}_{\mathrm{Cbz}^{\prime}} \mathrm{CH}_{2}\right), 5.28\left(\mathrm{~d}, J=12.3 \mathrm{~Hz}, 1 \mathrm{H}, \mathrm{Ph}_{\mathrm{Cbz}} \mathrm{CH}_{\mathrm{b}}\right), 4.29(\mathrm{dd}, J=2.9,10.2 \mathrm{~Hz}, 1 \mathrm{H}, \mathrm{C} 11 \mathrm{H}), 4.18-4.14(\mathrm{~m}, 1 \mathrm{H}$, $\left.\mathrm{C}_{11} \mathrm{H}\right), 4.14-4.08\left(\mathrm{~m}, 1 \mathrm{H}, \mathrm{C}_{15} \cdot \mathrm{H}\right), 4.05\left(\mathrm{app}-\mathrm{t}, J=8.0 \mathrm{~Hz}, 1 \mathrm{H}, \mathrm{C}_{15} \mathrm{H}\right), 3.67-3.50\left(\mathrm{~m}, 5 \mathrm{H}, \mathrm{C}_{12} \cdot \mathrm{H}_{\mathrm{a}}, \mathrm{C}_{19} \mathrm{H}, \mathrm{C}_{19} \cdot \mathrm{H}\right)$, $3.15\left(\mathrm{dd}, J=5.8,12.9 \mathrm{~Hz}, 1 \mathrm{H}, \mathrm{C}_{12} \mathrm{H}_{\mathrm{a}}\right), 2.92-2.81\left(\mathrm{~m}, 2 \mathrm{H}, \mathrm{C}_{12} \mathrm{H}_{\mathrm{b}}, \mathrm{C}_{12} \mathrm{H}_{\mathrm{b}}\right), 2.37-2.28(\mathrm{~m}, 2 \mathrm{H}), 2.27-2.15(\mathrm{~m}, 1 \mathrm{H}$, $\left.\mathrm{C}_{17} \cdot \mathbf{H}\right), 2.11-1.98\left(\mathrm{~m}, 3 \mathrm{H}, \mathrm{C}_{17} \mathrm{H}_{\mathrm{b}}, \mathrm{C}_{18} \mathrm{H}\right), 1.98-1.85\left(\mathrm{~m}, 2 \mathrm{H}, \mathrm{C}_{18} \cdot \mathbf{H}\right) \cdot{ }^{13} \mathrm{C} N M R\left(\mathrm{MHz}, \mathrm{CDCl}_{3}, 20{ }^{\circ} \mathrm{C}\right): \delta 169.4\left(\mathbf{C}_{16^{\prime}}\right)$, $166.0\left(\mathbf{C}_{16}\right), 165.4\left(\mathbf{C}_{13}\right), 165.0\left(\mathbf{C}_{13^{\prime}}\right), 153.1\left(\mathbf{C}_{\mathrm{Cbz}}=\mathrm{O}\right), 150.3\left(\mathbf{C}_{\mathrm{Cbz}}=\mathbf{O}\right), 141.5\left(\mathbf{C}_{9}\right), 138.8\left(\mathbf{C}_{7^{\prime}}\right), 136.2\left(2 \mathrm{C}, \mathbf{C}_{4^{\prime}}\right.$, $\left.\mathrm{Ph}_{\mathrm{Cbz}}-\mathrm{i}-\mathbf{C}\right), 134.9\left(3 \mathrm{C}, \mathbf{C}_{4}, \mathbf{C}_{9^{\prime}}, \mathrm{Ph}_{\mathrm{Cbz}^{\prime}}-i-\mathbf{C}\right), 129.3\left(\mathbf{C}_{7}\right), 129.0\left(2 \mathrm{C}, \mathrm{Ph}_{\mathrm{Cbz}}-\mathbf{C}\right), 128.8\left(\mathrm{Ph}_{\mathrm{Cbz}}-\mathbf{C}\right), 128.6\left(\mathrm{Ph}_{\mathrm{Cbz}}-\mathbf{C}\right)$, 
$128.3\left(\mathrm{Ph}_{\mathrm{Cbz}}-\mathbf{C}\right), 128.2\left(\mathrm{Ph}_{\mathrm{Cbz}}-\mathbf{C}\right), 124.8\left(\mathbf{C}_{2^{\prime}}\right), 124.7\left(\mathbf{C}_{7^{\prime}}\right), 124.0\left(\mathbf{C}_{5}\right), 121.1\left(\mathbf{C}_{6}\right), 119.5\left(\mathbf{C}_{5^{\prime}}\right), 117.6\left(\mathbf{C}_{8}\right), 115.7$ $\left(\mathbf{C}_{3^{\prime}}\right), 113.0\left(\mathbf{C}_{8^{\prime}}\right), 81.8\left(\mathbf{C}_{2}\right), 69.1\left(\mathrm{Ph}_{\mathrm{Cbz}^{\prime}}-\mathbf{C H}_{2}\right), 68.3\left(\mathrm{Ph}_{\mathrm{Cbz}}-\mathbf{C H}_{2}\right), 60.7\left(\mathbf{C}_{11}\right.$ or $\left.\mathbf{C}_{15^{\prime}}\right), 60.6\left(\mathbf{C}_{11}\right.$ or $\left.\mathbf{C}_{15^{\prime}}\right), 59.3\left(\mathbf{C}_{15}\right)$, $58.6\left(\mathbf{C}_{3}\right), 54.0\left(\mathbf{C}_{11^{\prime}}\right), 45.6\left(\mathbf{C}_{19}\right.$ or $\left.\mathbf{C}_{19^{\prime}}\right), 45.5\left(\mathbf{C}_{19}\right.$ or $\left.\mathbf{C}_{19^{\prime}}\right), 39.4\left(\mathbf{C}_{12}\right), 28.4\left(\mathbf{C}_{17^{\prime}}\right), 28.0\left(\mathbf{C}_{17}\right), 26.5\left(\mathbf{C}_{12^{\prime}}\right), 23.1$ $\left(\mathbf{C}_{18^{\prime}}\right), 22.7\left(\mathbf{C}_{18}\right)$. FTIR (thin film) $\mathrm{cm}^{-1}: 3472$ (br-m), 2955 (w), 1713 (s), 1667 (s), $1480(\mathrm{~m}), 1398$ (m). HRMS (ESI) $(\mathrm{m} / \mathrm{z})$ : calc'd for $\mathrm{C}_{48} \mathrm{H}_{45} \mathrm{~N}_{6} \mathrm{O}_{8}[\mathrm{M}+\mathrm{H}]^{+}:$833.3293, found: 833.3278. $[\alpha]_{\mathrm{D}}{ }^{24}:-166\left(c=0.26, \mathrm{CHCl}_{3}\right)$. TLC (70\% acetone in hexanes), Rf: 0.28 (UV, CAM). 

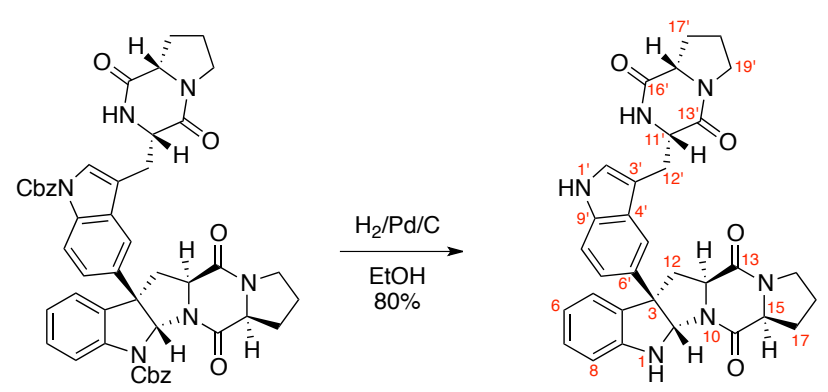

(-)-Naseseazine C (4): Palladium on activated charcoal (3.3 mg, $3.1 \mu \mathrm{mol}, 20 \mathrm{~mol} \%$ ) was added to a solution of para(-)-Nin,Nin'-dicarboxybenzyl naseseazine C (-)-9 (12.9 mg, $30.4 \mathrm{mmol}, 1$ equiv) in degassed $\left(\mathrm{N}_{2}\right.$ stream, $5 \mathrm{~min}$ ) ethanol (1.6 mL). A stream of hydrogen gas was passed through the heterogeneous mixture for 2 min by discharge of a balloon equipped with a needle extending into the reaction mixture. After stirring the reaction mixture at $22{ }^{\circ} \mathrm{C}$ for $21 \mathrm{~h}$ under an atmosphere of hydrogen gas, the solution was degassed $\left(\mathrm{N}_{2}\right.$ stream, $5 \mathrm{~min}$ ) and filtered through Celite. The filter cake was washed with methanol $(3 \times 2 \mathrm{~mL})$ and the combined filtrates were concentrated under reduced pressure. The crude reaction mixture was purified by flash column chromatography on silica gel (eluent: $70 \rightarrow 90 \%$ acetone in dichloromethane) to afford (-)-4 (7.0 mg, $80 \%)$. Structural assignments were made using additional information from gCOSY, HSQC, HMBC and nOe experiments.

1H NMR (MHz, CD3OD, $\left.20^{\circ} \mathrm{C}\right): \delta 7.64\left(\mathrm{~d}, \mathrm{~J}=1.8 \mathrm{~Hz}, 1 \mathrm{H}, \mathrm{C} 5^{\prime} \mathrm{H}\right), 7.24\left(\mathrm{~d}, \mathrm{~J}=8.6 \mathrm{~Hz}, 1 \mathrm{H}, \mathrm{C} 8^{\prime} \mathrm{H}\right), 7.19(\mathrm{~d}, \mathrm{~J}=$ $7.4 \mathrm{~Hz}, 1 \mathrm{H}, \mathrm{C} 5 \mathrm{H}), 7.13\left(\mathrm{dd}, \mathrm{J}=1.9,8.6 \mathrm{~Hz}, 1 \mathrm{H}, \mathrm{C} 7 \mathrm{\prime}^{\prime} \mathrm{H}\right), 7.08\left(\mathrm{~s}, 1 \mathrm{H}, \mathrm{C} 2{ }^{\prime} \mathrm{H}\right), 7.06(\mathrm{td}, \mathrm{J}=1.2,7.6 \mathrm{~Hz}, 1 \mathrm{H}, \mathrm{C} 7 \mathrm{H})$, $6.75(\mathrm{td}, \mathrm{J}=1.0,7.5 \mathrm{~Hz}, 1 \mathrm{H}, \mathrm{C} 6 \mathrm{H}), 6.71(\mathrm{~d}, \mathrm{~J}=8.1 \mathrm{~Hz}, 1 \mathrm{H}, \mathrm{C} 8 \mathrm{H}), 5.70(\mathrm{~s}, 1 \mathrm{H}, \mathrm{C} 2 \mathrm{H}), 4.39(\mathrm{td}, \mathrm{J}=1.8,4.9 \mathrm{~Hz}$, $\left.1 \mathrm{H}, \mathrm{C} 11^{\prime} \mathrm{H}\right), 4.31-4.23(\mathrm{~m}, 2 \mathrm{H}, \mathrm{C} 11 \mathrm{H}, \mathrm{C} 15 \mathrm{H}), 3.97$ (ddd, J = 1.9, 6.4, $\left.10.9 \mathrm{~Hz}, 1 \mathrm{H}, \mathrm{C} 15 \mathrm{H}\right), 3.54-3.47(\mathrm{~m}, 2 \mathrm{H}$, $\mathrm{C} 19 \mathrm{H}), 3.45-3.39\left(\mathrm{~m}, 1 \mathrm{H}, \mathrm{C} 19^{\prime} \mathrm{Ha}\right), 3.28$ (app-d, J = 5.2 Hz, 2H, C12'H), 3.26-3.21 (m, 1H, C19'Hb), 3.12 (dd, $\mathrm{J}=6.1,12.7 \mathrm{~Hz}, 1 \mathrm{H}, \mathrm{C} 12 \mathrm{Ha}), 2.95-2.88(\mathrm{~m}, 1 \mathrm{H}, \mathrm{C} 12 \mathrm{Hb}), 2.33-2.25(\mathrm{~m}, 1 \mathrm{H}, \mathrm{C} 17 \mathrm{Ha}), 2.10-2.00(\mathrm{~m}, 2 \mathrm{H}$, $\mathrm{C} 17 \mathrm{Hb}, \mathrm{C} 18 \mathrm{Ha}), 1.98-1.87\left(\mathrm{~m}, 2 \mathrm{H}, \mathrm{C} 17^{\prime} \mathrm{Ha}, \mathrm{C} 18 \mathrm{Hb}\right), 1.71-1.60\left(\mathrm{~m}, 1 \mathrm{H}, \mathrm{C} 18^{\prime} \mathrm{Ha}\right), 1.48-1.40\left(\mathrm{~m}, 1 \mathrm{H}, \mathrm{C} 18^{\prime} \mathrm{Hb}\right)$, 0.98-0.88 (m, 1H, C17'Hb). 1H NMR (MHz, DMSO-d6, $\left.20^{\circ} \mathrm{C}\right): \delta 10.84\left(\mathrm{~s}, 1 \mathrm{H}, \mathrm{N} 1{ }^{\prime} \mathrm{H}\right), 7.74\left(\mathrm{~s}, 1 \mathrm{H}, \mathrm{N} 10^{\prime} \mathrm{H}\right), 7.63$ (s, 1H, C5'H), 7.22, (d, J = 8.6 Hz, 1H, C8'H), $7.17\left(\mathrm{~s}, 1 \mathrm{H}, \mathrm{C} 8{ }^{\prime} \mathrm{H}\right), 7.16(\mathrm{~d}, \mathrm{~J}=8.7 \mathrm{~Hz}, 1 \mathrm{H}, \mathrm{C} 5 \mathrm{H}), 7.03(\mathrm{~d}, \mathrm{~J}=8.6$ $\left.\mathrm{Hz}, 1 \mathrm{H}, \mathrm{C} 7 \mathrm{H}^{\prime}\right), 6.98$ (app-t, J = 7.7 Hz, 1H, C7H), 6.65 (d, J = 8.6 Hz, C8H), $6.64(\mathrm{~s}, 1 \mathrm{H}, \mathrm{N} 1 \mathrm{H}), 6.61$ (app-t, J = $7.4 \mathrm{~Hz}, 1 \mathrm{H}, \mathrm{C} 6 \mathrm{H}), 5.63(\mathrm{~s}, 1 \mathrm{H}, \mathrm{C} 2 \mathrm{H}), 4.35-4.27\left(\mathrm{~m}, 2 \mathrm{H}, \mathrm{C} 111^{\prime} \mathrm{H}, \mathrm{C} 15 \mathrm{H}\right), 4.16(\mathrm{dd}, \mathrm{J}=6.1,11.4 \mathrm{~Hz}, 1 \mathrm{H}, \mathrm{C} 11 \mathrm{H})$, 4.09 (app-t, J = 8.4 Hz, 1H, C15'H), 3.45-3.33 (m, 3H, C19H, C19'Ha), 3.33-3.23 (m, 1H, C19'Hb), 3.22 (dd, J $=4.8,14.9 \mathrm{~Hz}, 1 \mathrm{H}, \mathrm{C} 12 \mathrm{Ha}), 3.10-3.02\left(\mathrm{~m}, 2 \mathrm{H}, \mathrm{C} 12 \mathrm{Ha}, \mathrm{C} 12^{\prime} \mathrm{Hb}\right), 2.68$ (app-t, J = $\left.12.0 \mathrm{~Hz}, 1 \mathrm{H}, \mathrm{C} 12 \mathrm{Hb}\right), 2.19-$ $2.08(\mathrm{~m}, 1 \mathrm{H}, \mathrm{C} 17 \mathrm{Ha}), 2.04-1.97(\mathrm{~m}, 1 \mathrm{H}, \mathrm{C} 17 \mathrm{Ha}), 1.97-1.86(\mathrm{~m}, 2 \mathrm{H}, \mathrm{C} 17 \mathrm{Hb}, \mathrm{C} 18 \mathrm{Ha}), 1.85-1.76(\mathrm{~m}, 1 \mathrm{H}$, $\mathrm{C} 18 \mathrm{Hb}), 1.75-1.68\left(\mathrm{~m}, 1 \mathrm{H}, \mathrm{C} 18^{\prime} \mathrm{Ha}\right), 1.68-1.59\left(\mathrm{~m}, 1 \mathrm{H}, \mathrm{C} 18^{\prime} \mathrm{Hb}\right), 1.53-1.42\left(\mathrm{~m}, 1 \mathrm{H}, \mathrm{C} 17^{\prime} \mathrm{Hb}\right) .13 \mathrm{C} \mathrm{NMR}(\mathrm{MHz}$, CD3OD, $20^{\circ} \mathrm{C}$ ): $\delta 170.7$ (C16'), 168.5 (C16), 168.0 (C13), 167.4 (C13'), 150.6 (C9), 136.9 (C9'), 135.3 (C6'), 134.2 (C4), 129.6 (C7), 128.5 (C4'), 126.2 (C2'), 125.2 (C5), 121.1 (C7'), 120.5 (C6), 116.3 (C5'), 112.6 (C8'), 111.1 (C8), 109.9 (C3'), 83.6 (C2), 61.9 (C15), 61.7 (C11), 60.8 (C3), 60.0 (C15'), 57.2 (C11'), 46.3 (C19), 46.0 (C19'), 41.1 (C12), 29.1 (2C, C12', C17'), 28.9 (C17), 23.8 (C18), 22.6 (C18'). 13C NMR (MHz, DMSO-d6, 20 $\left.{ }^{\circ} \mathrm{C}\right): \delta 169.2$ (C16'), 166.0 (C16), 165.6 (C13'), 165.3 (C13), 149.3 (C9), 134.8 (C9'), 134.1 (C6'), 133.0 (C4), 128.1 (C7), 127.1 (C4'), 124.8 (C2'), 123.8 (C5), 119.2 (C7'), 118.1 (C6), 114.6 (C5'), 111.4 (C8'), 109.6 (C3'), 109.5 (C8), 81.1 (C2), 60.1 (C11), 59.7 (C15), 58.7 (C3), 58.5 (C15'), 55.1 (C11'), 44.7 (2C, C19, C19'), 39.9 (C12), 27.7 (C17'), 27.4 (C17), 25.4 (C12'), 22.6 (C18), 22.0 (C18'). FTIR (thin film) cm-1: $\quad 3310 \quad$ (br-m), $2926(\mathrm{~m}), 1661$ (s), 1435 (s), 1301 (w), 1150 (w). HRMS (ESI) (m/z): calc'd for C32H33N6O4 $[\mathrm{M}+\mathrm{H}]+: 565.2558$, found: 565.2551 . [a]D24: $-180(\mathrm{c}=0.16, \mathrm{CH} 3 \mathrm{OH})$. TLC $(80 \%$ acetone in dichloromethane $)$, Rf: 0.11 (UV, CAM). 


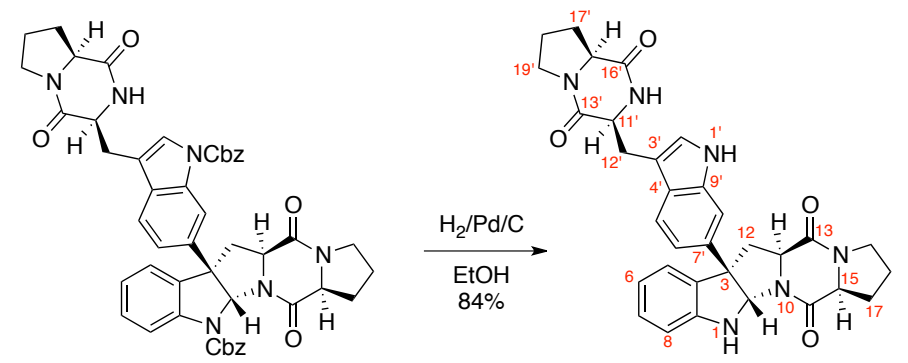

(-)-iso-Naseseazine C (11): Palladium on activated charcoal $(6.5 \mathrm{mg}, 6.1 \mu \mathrm{mol}, 20.0 \mathrm{~mol} \%)$ was added to a solution of meta(-)-Nin,Nin'-dicarboxybenzyl iso-naseseazine C (-)-10 (25.2 mg, $30.3 \mu \mathrm{mol}, 1$ equiv) in degassed $\left(\mathrm{N}_{2}\right.$ stream, $5 \mathrm{~min}$ ) ethanol $(3 \mathrm{~mL})$. A stream of hydrogen gas was passed through the heterogeneous mixture for 2 min by discharge of a balloon equipped with a needle extending into the reaction mixture. After stirring the reaction mixture at $22{ }^{\circ} \mathrm{C}$ for $64 \mathrm{~h}$ under an atmosphere of hydrogen gas, the solution was degassed ( $\mathrm{N}_{2}$ stream, $\left.5 \mathrm{~min}\right)$ and filtered over Celite. The filter cake was washed with methanol $(3 \times 2 \mathrm{~mL})$ and the combined filtrates were concentrated under reduced pressure. The crude reaction mixture was purified by flash column chromatography on silica gel (eluent: $70 \rightarrow 90 \%$ acetone in dichloromethane) to afford (-)-11 (14.3 mg, 83.6\%). Structural assignments were made using additional information from gCOSY, HSQC, HMBC and nOe experiments.

1H NMR (MHz, CD3OD, $\left.20^{\circ} \mathrm{C}\right): \delta 7.49\left(\mathrm{~d}, \mathrm{~J}=8.4 \mathrm{~Hz}, 1 \mathrm{H}, \mathrm{C} 5^{\prime} \mathrm{H}\right), 7.31\left(\mathrm{~d}, \mathrm{~J}=1.6 \mathrm{~Hz}, 1 \mathrm{H}, \mathrm{C} 8^{\prime} \mathrm{H}\right), 7.11-7.01$ $\left(\mathrm{m}, 2 \mathrm{H}, \mathrm{C} 5 \mathrm{H}, \mathrm{C}^{\prime} \mathrm{H}\right), 7.06(\mathrm{~s}, 1 \mathrm{H}, \mathrm{C} 2 \mathrm{H}), 7.05$ (td, J = 1.1, 7.1 Hz, 1H, C7H), 6.72 (td, J = 1.0, 7.5 Hz, 1H, C6H), $6.70(\mathrm{~d}, \mathrm{~J}=7.7 \mathrm{~Hz}, 1 \mathrm{H}, \mathrm{C} 8 \mathrm{H}), 5.64(\mathrm{~s}, 1 \mathrm{H}, \mathrm{C} 2 \mathrm{H}), 4.35\left(\mathrm{td}, \mathrm{J}=1.8,5.0 \mathrm{~Hz}, 1 \mathrm{H}, \mathrm{C} 11^{\prime} \mathrm{H}\right), 4.21-4.15(\mathrm{~m}, 2 \mathrm{H}$, $\mathrm{C} 11 \mathrm{H}, \mathrm{C} 15 \mathrm{H}), 3.92$ (ddd, J = 1.9, 6.5, $11.0 \mathrm{~Hz}, 1 \mathrm{H}, \mathrm{C} 15 \mathrm{H}), 3.54-3.44(\mathrm{~m}, 2 \mathrm{H}, \mathrm{C} 19 \mathrm{H}), 3.44-3.37(\mathrm{~m}, 1 \mathrm{H}$, C19'Ha), 3.26 (app-d, J = 4.5 Hz, 2H, C12'H), 3.24-3.19 (m, 1H, C19'Hb), 3.05 (dd, J = 6.0, 12.6 Hz, 1H, $\mathrm{C} 12 \mathrm{Ha}), 2.85-2.78(\mathrm{~m}, 1 \mathrm{H}, \mathrm{C} 12 \mathrm{Hb}), 2.29-2.21(\mathrm{~m}, 1 \mathrm{H}, \mathrm{C} 17 \mathrm{Ha}), 2.08-1.97(\mathrm{~m}, 2 \mathrm{H}, \mathrm{C} 17 \mathrm{Hb}, \mathrm{C} 18 \mathrm{Ha}), 1.95-1.85$ (m, 2H, C17'Ha, C18Hb), 1.67-1.56 (m, 1H, C18'Ha), 1.46-1.30 (m, 1H, C18'Hb), 0.96-0.86 (m, 1H, C17'Hb). $1 \mathrm{H}$ NMR (MHz, DMSO-d6, $\left.20^{\circ} \mathrm{C}\right): \delta 10.79$ (d, J = 2.5 Hz, 1H, N1'H), $7.68\left(\mathrm{~s}, 1 \mathrm{H}, \mathrm{N} 10^{\prime} \mathrm{H}\right), 7.50$ (d, J = 8.4 Hz, $\left.1 \mathrm{H}, \mathrm{C}^{\prime} \mathrm{H}\right), 7.23\left(\mathrm{~d}, \mathrm{~J}=1.6 \mathrm{~Hz}, 1 \mathrm{H}, \mathrm{C} 8^{\prime} \mathrm{H}\right), 7.17$ (d, J = $\left.7.4 \mathrm{~Hz}, 1 \mathrm{H}, \mathrm{C} 5 \mathrm{H}\right), 7.15\left(\mathrm{~d}, \mathrm{~J}=2.4 \mathrm{~Hz}, 1 \mathrm{H}, \mathrm{C} 2 \mathrm{H}^{\prime}\right), 7.08$ (dd, J = 1.8, 8.3 Hz, 1H, C6'H), 6.99 (app-t, J = 7.8 Hz, 1H, C7H), 6.65 (d, J = 4.7 Hz, 1H, C8H), 6.65 (s, $1 \mathrm{H}$, $\mathrm{N} 1 \mathrm{H}), 6.63(\mathrm{~d}, \mathrm{~J}=7.5 \mathrm{~Hz}, 1 \mathrm{H}, \mathrm{C} 6 \mathrm{H}), 5.56(\mathrm{~s}, 1 \mathrm{H}, \mathrm{C} 2 \mathrm{H}), 4.31$ (ddd, J = 1.8, 6.9, $9.1 \mathrm{~Hz}, 1 \mathrm{H}, \mathrm{C} 15 \mathrm{H}), 4.26$ (app-t, $\left.\mathrm{J}=5.3 \mathrm{~Hz}, 1 \mathrm{H}, \mathrm{C} 11^{\prime} \mathrm{H}\right), 4.17(\mathrm{ddd}, \mathrm{J}=3.2,6.6,10.1 \mathrm{~Hz}, 1 \mathrm{H}, \mathrm{C} 11 \mathrm{H}), 4.05\left(\mathrm{dd}, \mathrm{J}=6.9,9.7 \mathrm{~Hz}, 1 \mathrm{H}, \mathrm{C} 15^{\prime} \mathrm{H}\right)$, 3.45-3.30 (m, 3H, C19'Ha, C19H), 3.25 (td, J = 4.3, 7.7, 8.3 Hz, 1H, C19'Hb), 3.20 (app-dd, J = 4.9, $15.0 \mathrm{~Hz}$, 1H, C12'Ha), 3.03 (app-ddd, J = 6.1, 13.8, $21.2 \mathrm{~Hz}, 2 \mathrm{H}, \mathrm{C} 12 \mathrm{Hb}, \mathrm{C} 12 \mathrm{Ha}), 2.70-2.61$ (m, 1H, C12Hb), 2.19$2.07(\mathrm{~m}, 1 \mathrm{H}, \mathrm{C} 17 \mathrm{Ha}), 2.01-1.86(\mathrm{~m}, 3 \mathrm{H}, \mathrm{C} 17 \mathrm{H}, \mathrm{C} 18 \mathrm{Ha}), 1.86-1.73(\mathrm{~m}, 1 \mathrm{H}, \mathrm{C} 18 \mathrm{Hb}), 1.73-1.63(\mathrm{~m}, 1 \mathrm{H}$, C18'Ha), 1.63-1.56 (m, 1H, C18'Hb), 1.46-1.35 (m, 1H, C17'Hb). 13C NMR (MHz, CD3OD, $\left.20^{\circ} \mathrm{C}\right): \delta 170.8$ (C16'), 168.5 (C16), 168.0 (C13), 167.4 (C13'), 150.6 (C9), 138.2 (C7'), 137.9 (C9'), 133.8 (C4), 129.7 (C7), 127.6 (C4'), 126.1 (C2'), 125.1 (C5), 120.4 (C5' or C6), 120.3 (C5' or C6), 118.0 (C6'), 111.1 (C8), 109.5 (C3'), 109.3 (C8'), 83.5 (C2), 61.8 (C11), 61.6 (C15), 60.7 (C3), 60.0 (C15'), 57.1 (C11'), 46.3 (C19), 46.0 (C19'), 40.9 (C12), 29.1 (C12' or C17'), 29.0 (C12' or C17'), 28.8 (C17), 23.8 (C18), 22.5 (C18'). 13C NMR (MHz, DMSO-d6, $20^{\circ} \mathrm{C}$ ): $\delta 169.1$ (C16'), 166.1 (C16), 165.5 (C13'), 165.3 (C13), 149.4 (C9), 136.8 (C7'), 135.9 (C9'), 132.4 (C4), 128.2 (C7), 126.1 (C4'), 124.8 (C2'), 124.0 (C5), 119.0 (C5'), 118.1 (C6), 116.4 (C6'), 109.5 (C8), 109.2 (C3'), 108.0 (C8'), 81.5 (C2), 60.2 (C11), 59.6 (C15), 58.8 (C3), 58.4 (C15'), 55.2 (C11'), 44.8 (C19 or C19'), 44.6 (C19 or C19'), 39.9 (C12), 27.6 (C17'), 27.4 (C17), 25.7 (C12'), 22.6 (C18), 21.9 (C18'). FTIR (thin film) cm-1: 3310 (br-m), 2955 (w), 1653 (s), 1457 (m), 1340 (w), 1056 (w). HRMS (ESI) (m/z): calc'd for C32H33N6O4 [M+H]+: 565.2558, found: 565.2554. [a]D24: -312 (c=0.42, CH3OH). TLC (80\% acetone in dichloromethane), Rf: 0.11 (UV, CAM). 
Supplementary Table 6. Comparison of our ${ }^{13} \mathrm{C}$ NMR data for (-)-naseseazine $C(4)$ and (-)-iso-naseseazine $\mathrm{C}$ (11) with literature data $\left(\mathrm{CD}_{3} \mathrm{OD}\right){ }^{1}$

\begin{tabular}{|c|c|c|c|c|c|}
\hline Assignment & $\begin{array}{c}\text { Wang Report }^{1} \\
\text { iso-naseseazine } \\
\mathrm{B} \\
{ }^{13} \mathrm{C} \mathrm{NMR}, 150 \\
\mathrm{MHz}, \mathrm{CD}_{3} \mathrm{OD}\end{array}$ & $\begin{array}{c}\text { This Work } \\
(-) \text {-naseseazine } \mathrm{C} \\
(4) \\
{ }^{13} \mathrm{C} \mathrm{NMR,}, 125 \mathrm{MHz} \\
\mathrm{CD}_{3} \mathrm{OD}, 20^{\circ} \mathrm{C}\end{array}$ & $\begin{array}{c}\text { This Work } \\
(-)-\text {-iso- } \\
\text { naseseazine } \mathrm{C} \\
(\mathbf{1 1}) \\
{ }^{13} \mathrm{C} N M R, 125 \mathrm{MHz} \\
\mathrm{CD}_{3} \mathrm{OD}, 20^{\circ} \mathrm{C}\end{array}$ & $\begin{array}{c}\text { Chemical Shift } \\
\text { Difference } \Delta \delta= \\
\delta(-) \text {-naseseazine } \\
C \text { (this work) }-\delta \\
\text { (Wang report) }^{1}\end{array}$ & $\begin{array}{c}\text { Chemical Shift } \\
\text { Difference } \\
\Delta \delta= \\
\delta(-)-i s o- \\
\text { naseseazine C } \\
\text { (this work) }-\delta^{\text {(Wang report) }}\end{array}$ \\
\hline $\mathrm{C} 2$ & 83.6 & 83.6 & 83.5 & 0 & -0.1 \\
\hline C3 & 60.8 & 60.8 & 60.7 & 0 & -0.1 \\
\hline C4 & 134.2 & 134.2 & 133.8 & 0 & -0.4 \\
\hline C5 & 125.2 & 125.2 & 125.1 & 0 & -0.1 \\
\hline C6 & 120.5 & 120.5 & 120.4 or 120.3 & 0 & -0.1 or -0.2 \\
\hline C7 & 129.7 & 129.6 & 129.7 & -0.1 & 0 \\
\hline C8 & 111.2 & 111.1 & 111.1 & -0.1 & -0.1 \\
\hline C9 & 150.6 & 150.6 & 150.6 & 0 & 0 \\
\hline C11 & 62.0 & 61.9 & 61.8 & -0.1 & -0.2 \\
\hline C12 & 41.1 & 41.1 & 40.9 & 0 & -0.2 \\
\hline C13 & 168.1 & 168.0 & 168.0 & -0.1 & -0.1 \\
\hline C15 & 61.7 & 61.9 & 61.6 & 0.2 & -0.1 \\
\hline C16 & 168.6 & 168.5 & 168.5 & -0.1 & -0.1 \\
\hline C17 & 28.9 & 28.9 & 28.8 & 0 & -0.1 \\
\hline C18 & 23.9 & 23.8 & 23.8 & -0.1 & -0.1 \\
\hline C19 & 46.4 & 46.3 & 46.3 & -0.1 & -0.1 \\
\hline $\mathrm{C} 2^{\prime}$ & 126.3 & 126.2 & 126.1 & -0.1 & -0.2 \\
\hline C3' & 109.8 & 109.9 & 109.5 & 0.1 & -0.3 \\
\hline C4' & 128.5 & 128.5 & 127.6 & 0 & -0.9 \\
\hline C5' & 112.6 & 116.3 & 120.4 or 120.3 & $3.7^{*}$ & 7.8 or 7.7 \\
\hline C6' & 121.1 & 135.3 & 118.0 & 14.2 & -3.1 \\
\hline C7' & 135.3 & 121.1 & 138.2 & -14.2 & 2.9 \\
\hline C8' & 116.3 & 112.6 & 109.3 & -3.7 & -7.0 \\
\hline C9' & 136.9 & 136.9 & 137.9 & 0 & 1.0 \\
\hline C11' & 57.3 & 57.2 & 57.1 & -0.1 & -0.2 \\
\hline C12' & 29.3 & 29.1 & 29.1 or 29.0 & -0.2 & -0.2 or -0.3 \\
\hline C13' & 167.4 & 167.4 & 167.4 & 0 & 0 \\
\hline C15' & 60.1 & 60.0 & 60.0 & -0.1 & 0.1 \\
\hline C16' & 170.7 & 170.7 & 170.8 & 0 & 0.1 \\
\hline C17' & 29.1 & 29.1 & 29.1 or 29.0 & 0 & 0 or -0.1 \\
\hline C18' & 22.5 & 22.6 & 22.5 & 0.1 & 0 \\
\hline C19' & 46.03 & 46.0 & 46.0 & 0 & 0 \\
\hline
\end{tabular}

The comparison of the ${ }^{1} \mathrm{H}$ and ${ }^{13} \mathrm{C}$ NMR chemical shifts of the reported isonaseseazine $\mathrm{B}$ with both synthetic regioisomers (-)-4 and (-)-11 in $\mathrm{CD}_{3} \mathrm{OD}$ shows that the data for isonaseseazine $\mathrm{B}$ is most consistent with synthetic (-)-naseseazine C (4).

* Our assignment of these residues is supported by key HSQC and HMBC correlations 
Supplementary Table 7. Comparison of our ${ }^{13} \mathrm{C}$ NMR data for (-)-naseseazine $C(4)$ and (-)-iso-naseseazine $\mathrm{C}(11)$ with literature data $\left(\mathrm{DMSO}-d_{6}\right)^{2}$

\begin{tabular}{|c|c|c|c|c|c|}
\hline Assignment & $\begin{array}{c}\text { Carroll } \\
\text { Report }^{2} \\
\text { naseseazine C } \\
\text { 13C NMR, 200 } \\
\text { MHz, DMSO-d6 }\end{array}$ & $\begin{array}{c}\text { This Work } \\
\text { (-)-naseseazine } \\
\mathrm{C}(4) 13 \mathrm{C} \mathrm{NMR} \\
125 \mathrm{MHz}\end{array}$ & 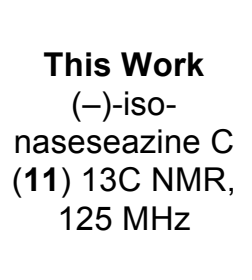 & $\begin{array}{c}\text { Chemical Shift } \\
\text { Difference } \\
\Delta \delta=\delta(-)- \\
\text { naseseazine C (this } \\
\text { work) }-\delta \text { (Carroll } \\
\text { report) }{ }^{2} \text { DMSO-d6, } \\
20^{\circ} \mathrm{C}\end{array}$ & $\begin{array}{c}\text { Chemical Shift } \\
\text { Difference } \\
\Delta \delta=\delta(-) \text {-iso- } \\
\text { naseseazine } C \text { (this work) } \\
-\delta(\text { Carroll report) } \\
\text { DMSO-d6, } 20^{\circ} \mathrm{C}\end{array}$ \\
\hline $\mathrm{C} 2$ & 81.1 & 81.1 & 81.5 & 0 & -0.4 \\
\hline C3 & 59.7 & 58.7 & 58.8 & $-1.0^{*}$ & 0.9 \\
\hline $\mathrm{C} 4$ & 134.1 & 133 & 132.4 & -1.1 & 1.7 \\
\hline $\mathrm{C} 5$ & 125.8 & 123.8 & 124 & -2.0 & 1.8 \\
\hline $\mathrm{C} 6$ & 118.1 & 118.1 & 118.1 & 0 & 0 \\
\hline $\mathrm{C7}$ & 128.1 & 128.1 & 128.2 & 0 & -0.1 \\
\hline $\mathrm{C} 8$ & 111.4 & 109.5 & 109.5 & -1.9 & 1.9 \\
\hline $\mathrm{C9}$ & 149.3 & 149.3 & 149.4 & 0 & -0.1 \\
\hline $\mathrm{C} 11$ & 60.1 & 60.1 & 60.2 & 0 & -0.1 \\
\hline C12 & 40 & 39.9 & 39.9 & -0.1 & 0.1 \\
\hline C13 & 165.3 & 165.3 & 165.3 & 0 & 0 \\
\hline C15 & 58.5 & 59.7 & 59.6 & 1.2 & -1.1 \\
\hline C16 & 169.1 & 166 & 166.1 & -3.1 & 3 \\
\hline C17 & 27.4 & 27.4 & 27.4 & 0 & 0 \\
\hline C18 & 22.5 & 22.6 & 22.6 & 0.1 & -0.1 \\
\hline C19 & 44.7 & 44.7 & 44.8 or 44.6 & 0 & 0.1 or -0.1 \\
\hline $\mathrm{C} 2^{\prime}$ & 124.8 & 124.8 & 124.8 & 0 & 0 \\
\hline C3' & 109.6 & 109.6 & 109.2 & 0 & 0.4 \\
\hline C4' & 127.1 & 127.1 & 126.1 & 0 & 1 \\
\hline C5' & 114.6 & 114.6 & 119 & 0 & -4.4 \\
\hline C6' & 134.8 & 134.1 & 116.4 & -0.7 & 18.4 \\
\hline C7' & 119.2 & 119.2 & 136.8 & 0 & -17.6 \\
\hline C8' & 110.4 & 111.4 & 108 & 1.0 & 2.4 \\
\hline C9' & 135.9 & 134.8 & 135.9 & -1.1 & 0 \\
\hline C11' & 55.1 & 55.1 & 55.2 & 0 & -0.1 \\
\hline C12' & 25.4 & 25.4 & 25.7 & 0 & -0.3 \\
\hline C13' & 166 & 165.6 & 165.5 & -0.4 & 0.5 \\
\hline C15' & 58.8 & 58.5 & 58.4 & -0.3 & 0.4 \\
\hline C16' & 165.6 & 169.2 & 169.1 & 3.6 & -3.5 \\
\hline C17' & 27.7 & 27.7 & 27.6 & 0 & 0.1 \\
\hline C18' & 21.9 & 22 & 21.9 & 0.1 & 0 \\
\hline C19' & 44.7 & 44.7 & 44.8 or 44.6 & 0 & 0.1 or -0.1 \\
\hline
\end{tabular}

The comparison of the ${ }^{1} \mathrm{H}$ and ${ }^{13} \mathrm{C}$ NMR chemical shifts of the reported naseseazine $\mathrm{C}$ with both synthetic regioisomers (-)-4 and (-)-11 in DMSO-d6 shows that the data for naseseazine $C$ is most consistent with synthetic (-)-naseseazine C (4).

* Our assignment of these residues is supported by key HSQC and HMBC correlations 
Synthetic References:

1. Xiong, Z. Q.; Liu, Q. X.; Pan, Z. L.; Zhao, N.; Feng, Z. X.; Wang, Y., Diversity and bioprospecting of culturable actinomycetes from marine sediment of the Yellow Sea, China. Arch. Microbiol. 2015, 197 (2), 299-309.

2. Buedenbender, L.; Grkovic, T.; Duffy, S.; Kurtboke, D. I.; Avery, V. M.; Carroll, A. R., Naseseazine C, a new anti-plasmodial dimeric diketopiperazine from a marine sediment derived Streptomyces sp. Tet. Lett. 2016, 57 (52), 5893-5895. 


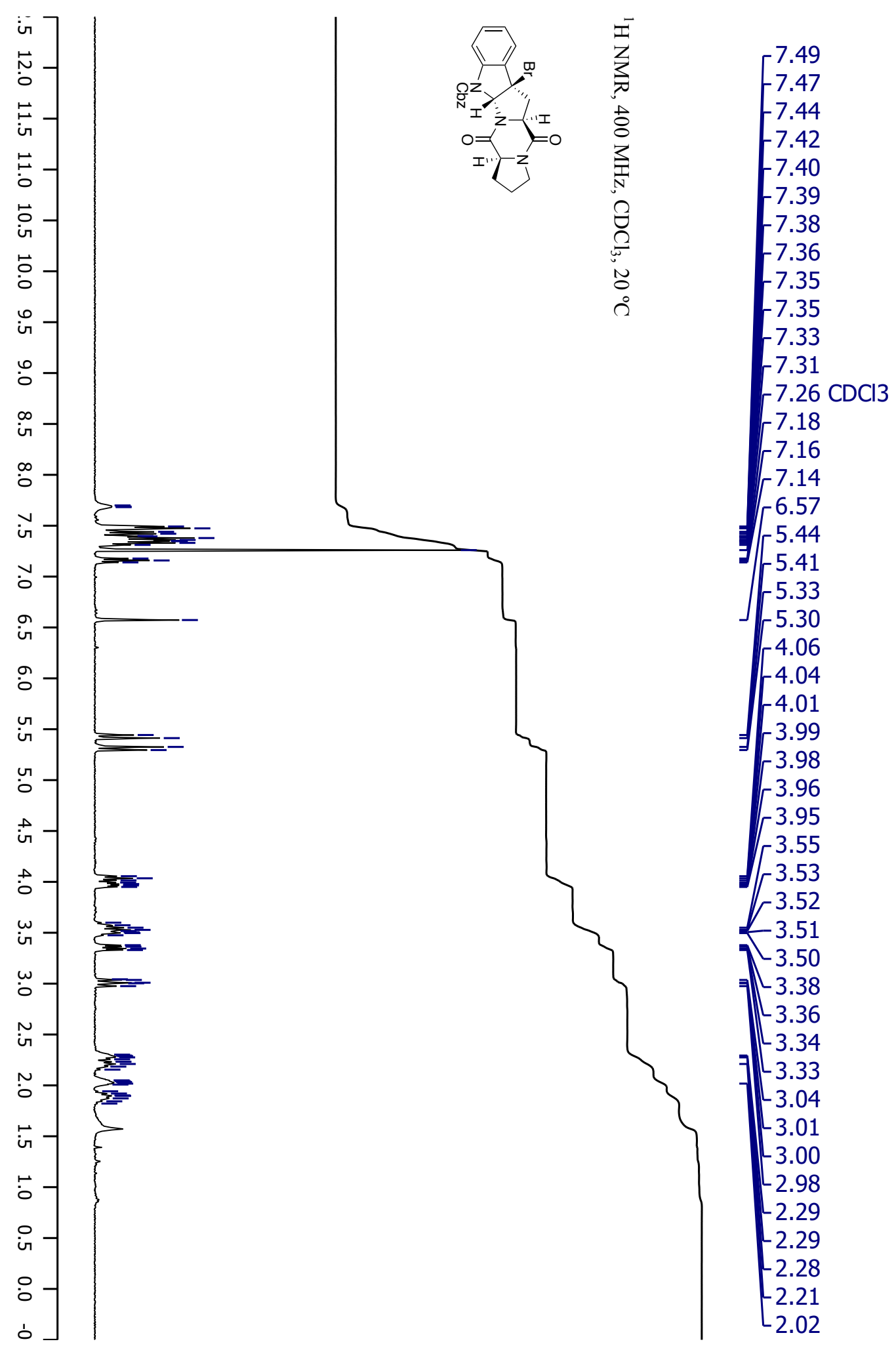




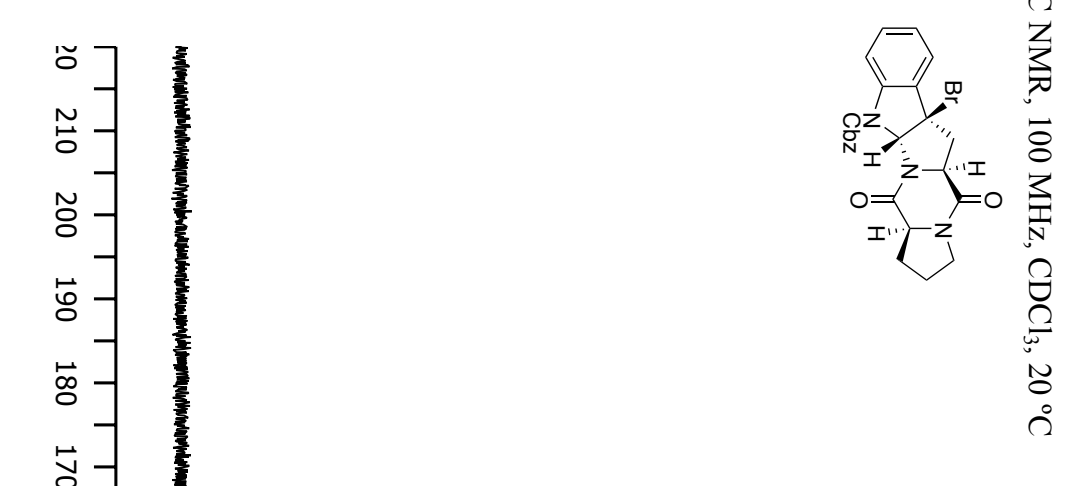

$-165.79$

$\sim_{164.20}$

$-152.96$

141.36

135.84

132.02

131.19

$-128.67$

- 128.46

128.40

125.10

123.88

- 117.67

꿍ㅁㅇㅇ

๖

$\varnothing$

๖

a

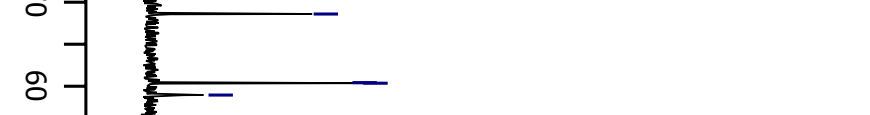

$-83.26$

$-77.16 \mathrm{CDCl} 3$

68.60

60.43

-60.36

58.95

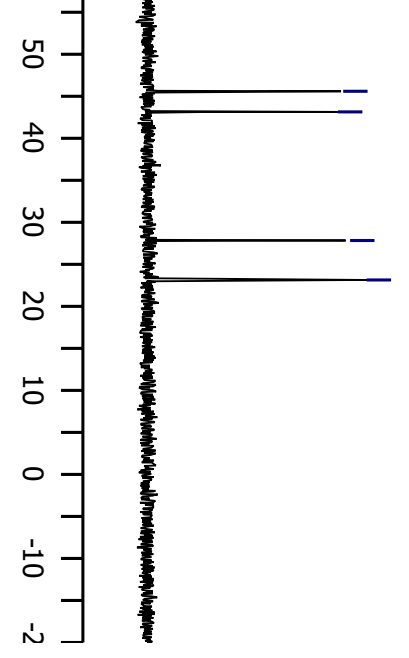

-45.60
-43.14
-27.84
-23.14

贅 

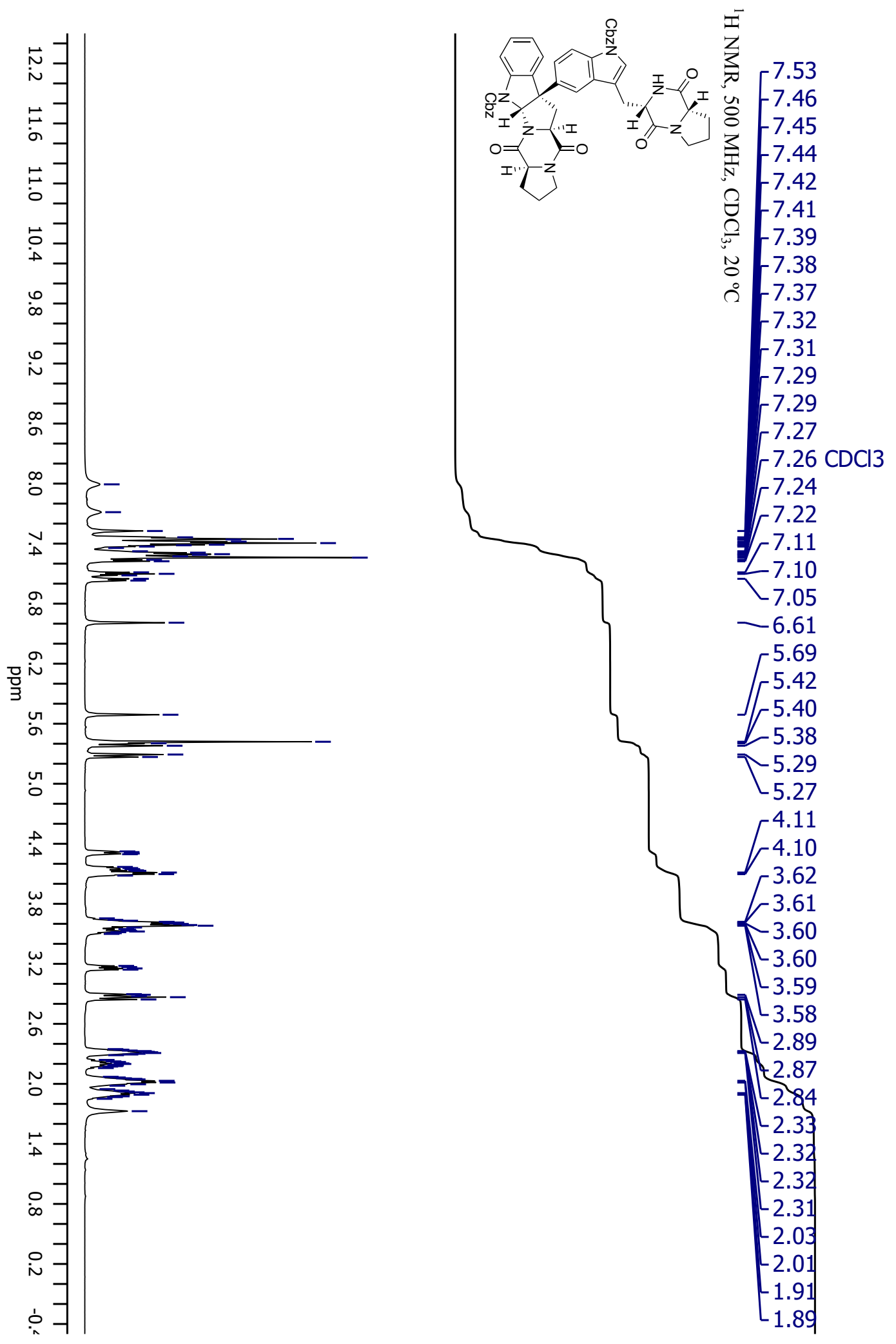

$\stackrel{\sim}{\circ}$ 

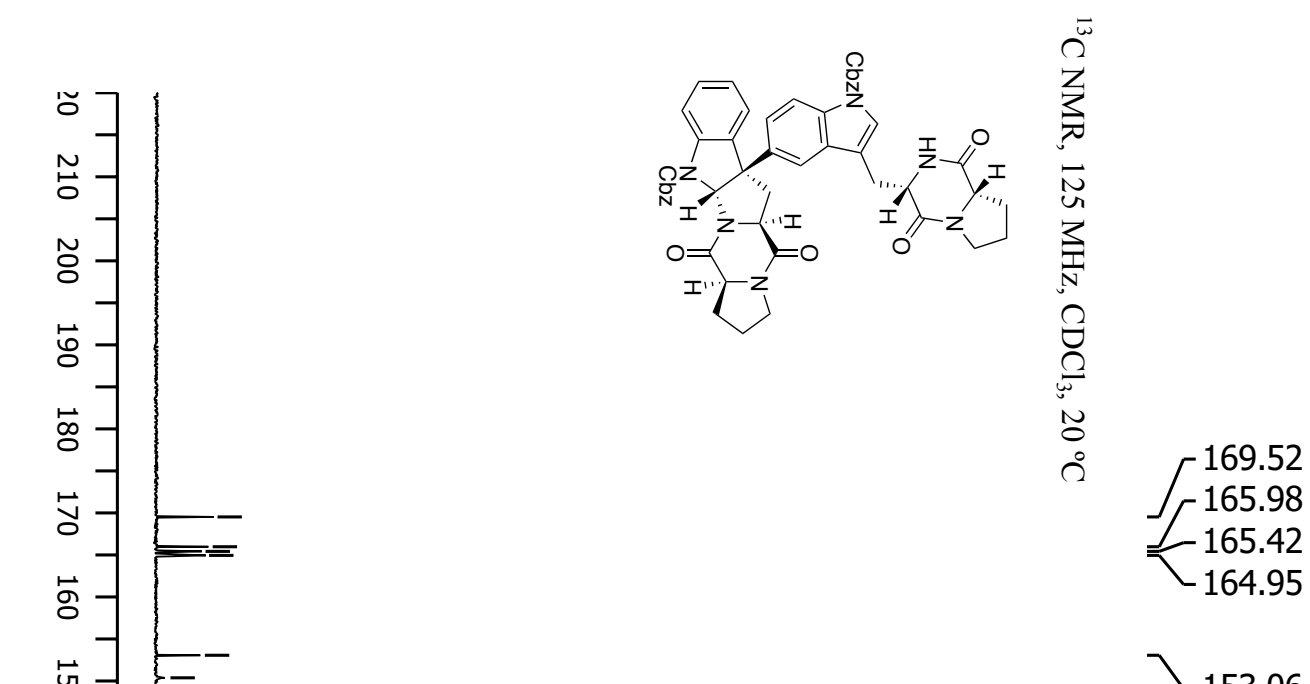

$\chi_{153.06}$

136.59
-136.10

134.80

129.34

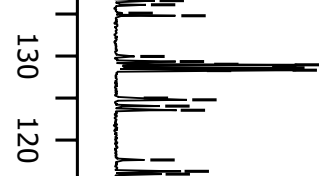

$-129.02$

$-128.96$

128.76

$-128.60$

$-128.32$

$-128.27$

$-124.79$

$-124.04$

=

ס

=

$-115.95$

$-115.43$

目 81.93

$77.16 \mathrm{CDCl} 3$

69.21

68.25

$-60.70$

$-60.60$

$-59.31$

7) -58.20

$-53.94$

$-45.64$

$-45.49$

$-39.63$

28.37

$-27.94$

$-26.47$

$-23.14$ 


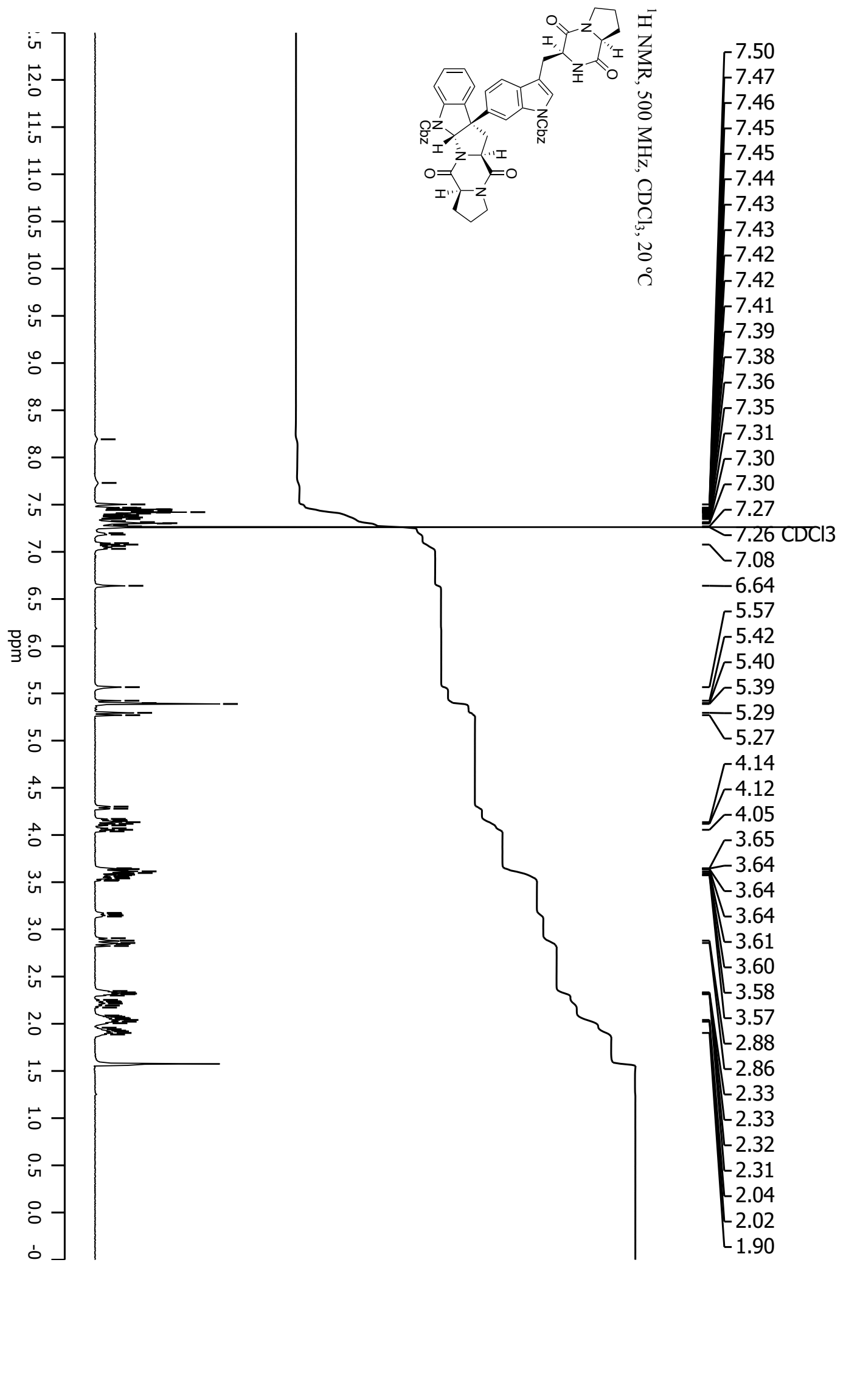



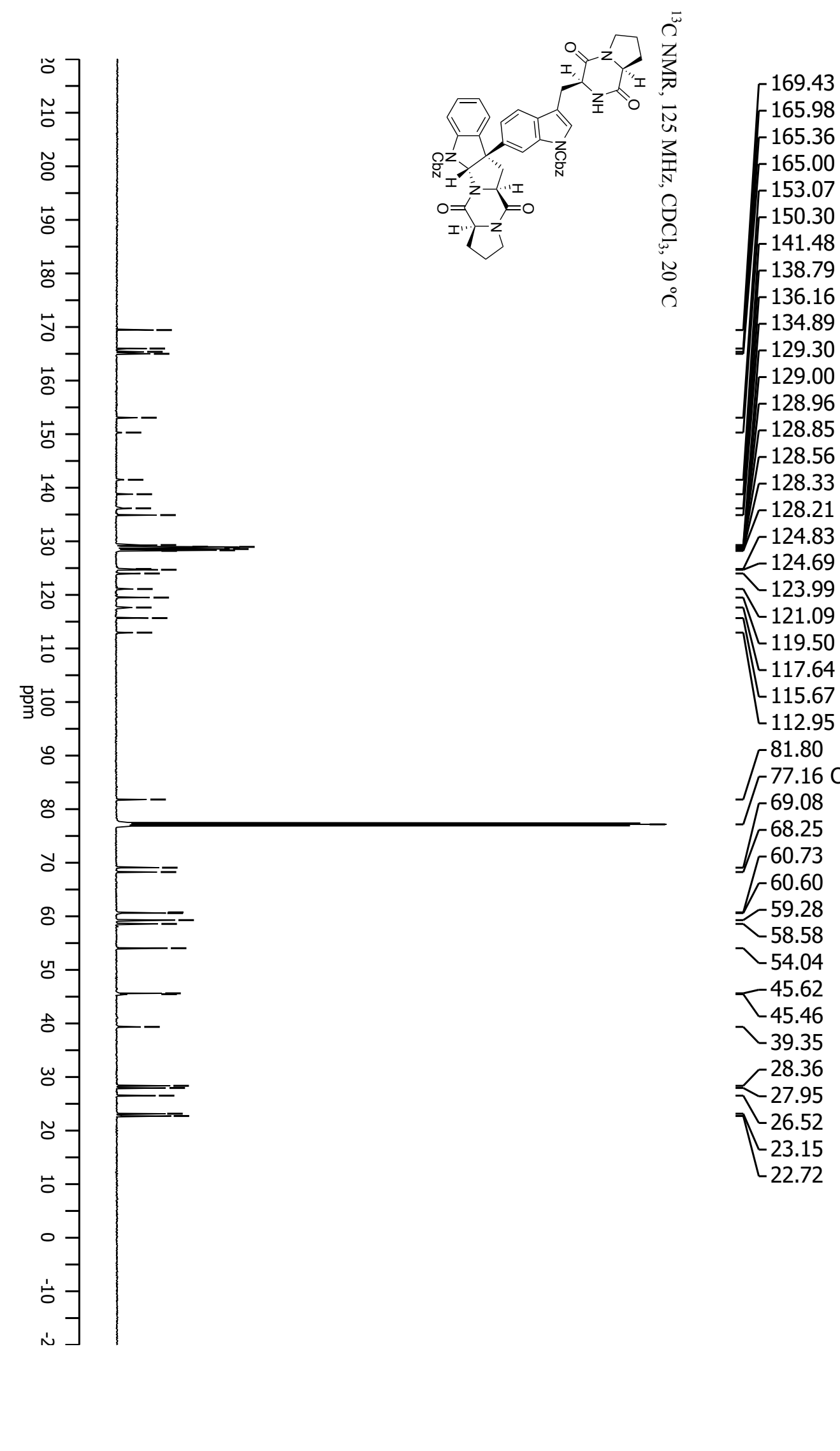

$-136.16$

$-134.89$

$-129.30$

$-129.00$

$-128.96$

$-128.85$

$-128.56$

128.33

$-128.21$

124.83

$-124.69$

-123.99

$-121.09$

- 119.50

117.64

115.67

$L_{112.95}$

81.80

$77.16 \mathrm{CDCl} 3$

69.08

68.25

60.73

60.60

$-59.28$

$-58.58$

$\backslash 54.04$

$-45.62$

$-45.46$

$\backslash 39.35$

28.36

$-27.95$

$-26.52$

$-23.15$

-22.72 


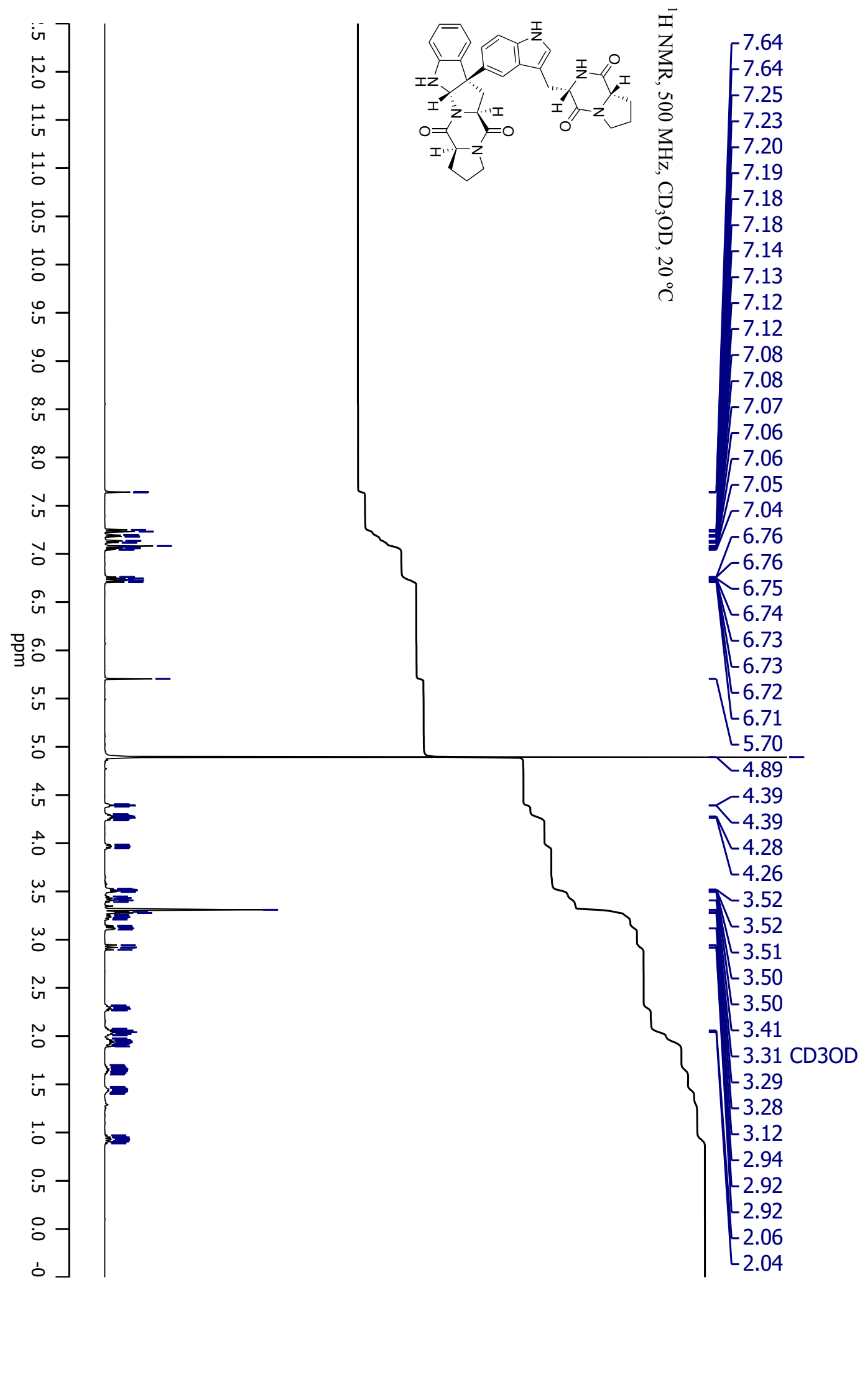



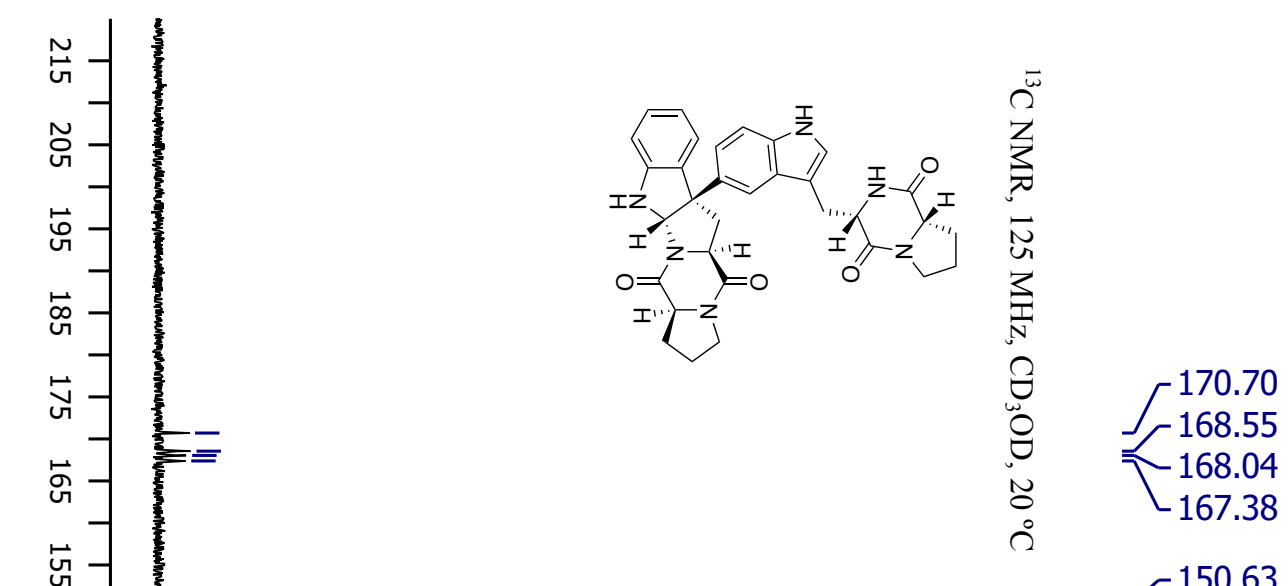

150.63

136.94

135.31

134.16

129.65

$-128.51$

- 126.17

125.18

$-121.07$

- 120.47

- 116.26

-112.59

111.12

$L_{109.86}$

$-83.59$

61.93

$-61.69$

-60.75

60.04

57.19

49.51

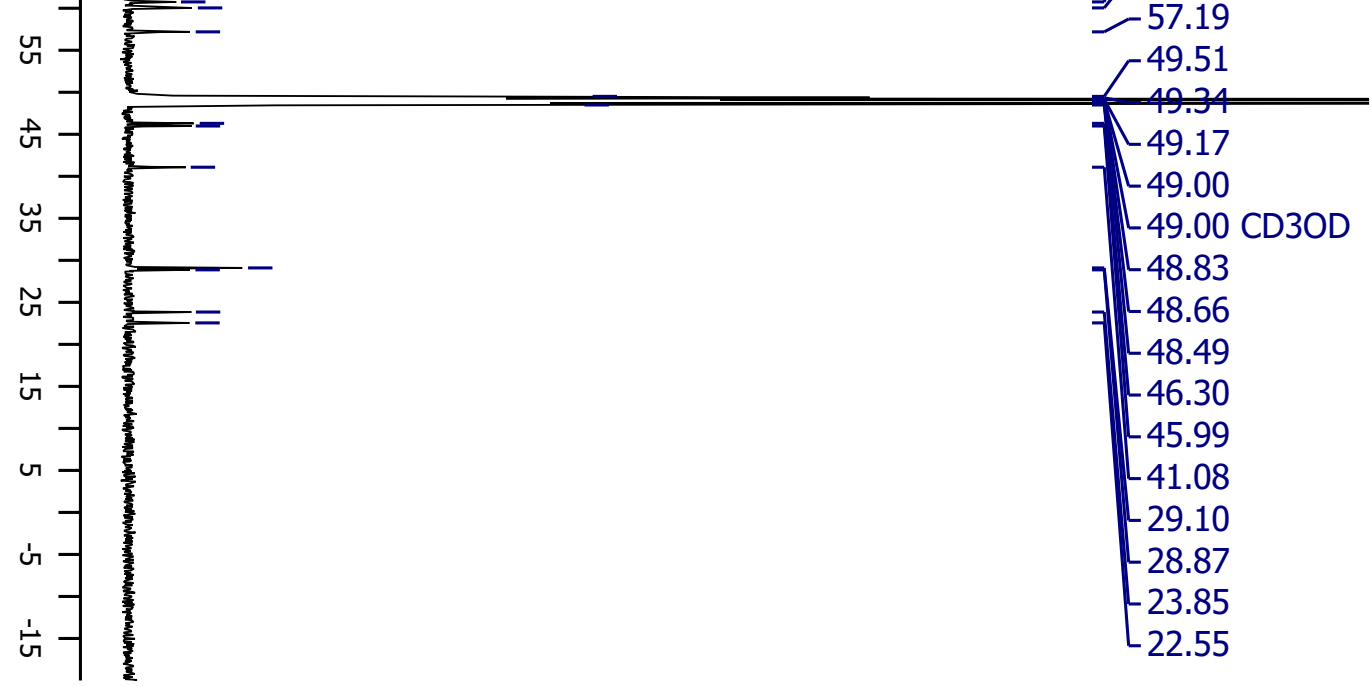




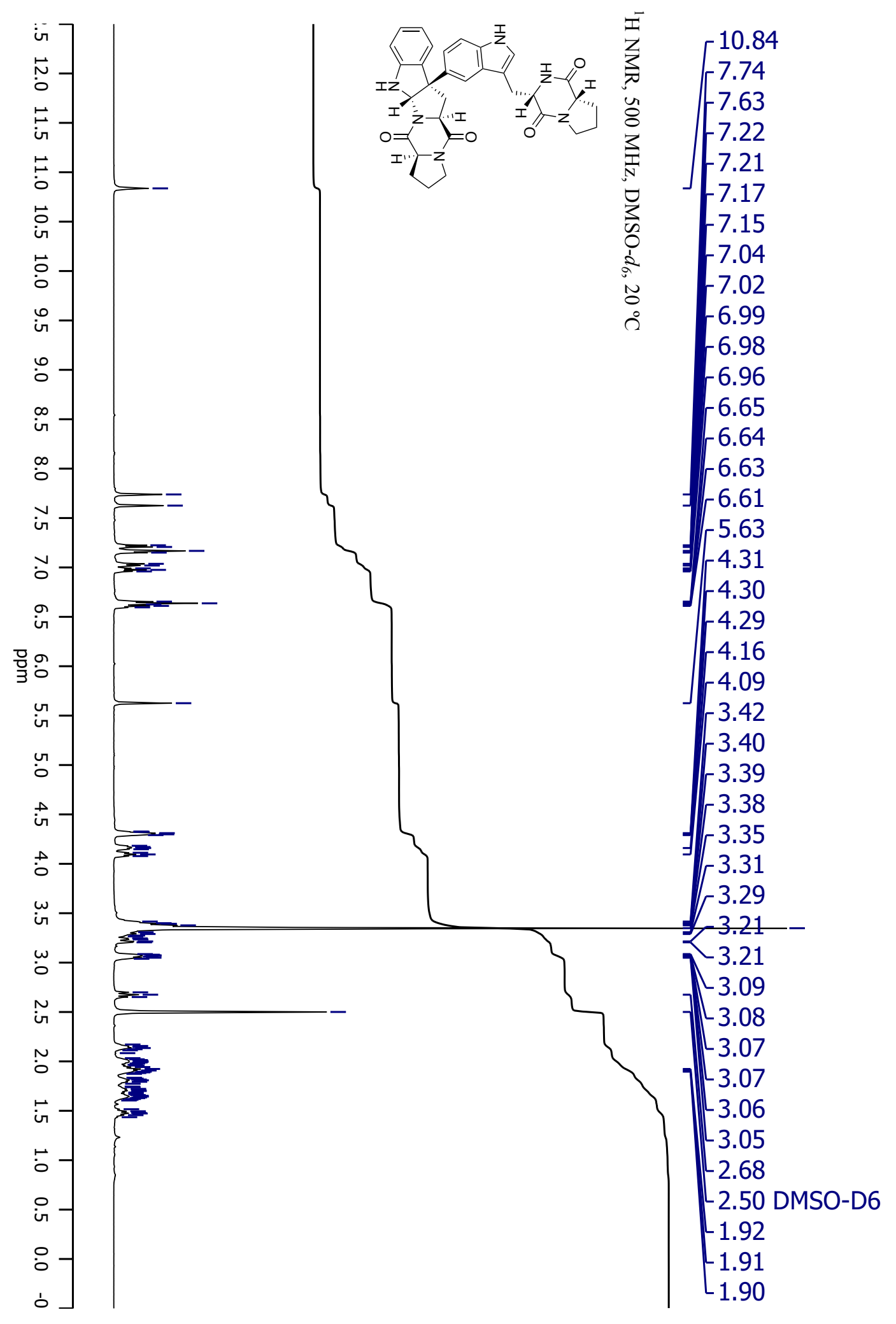



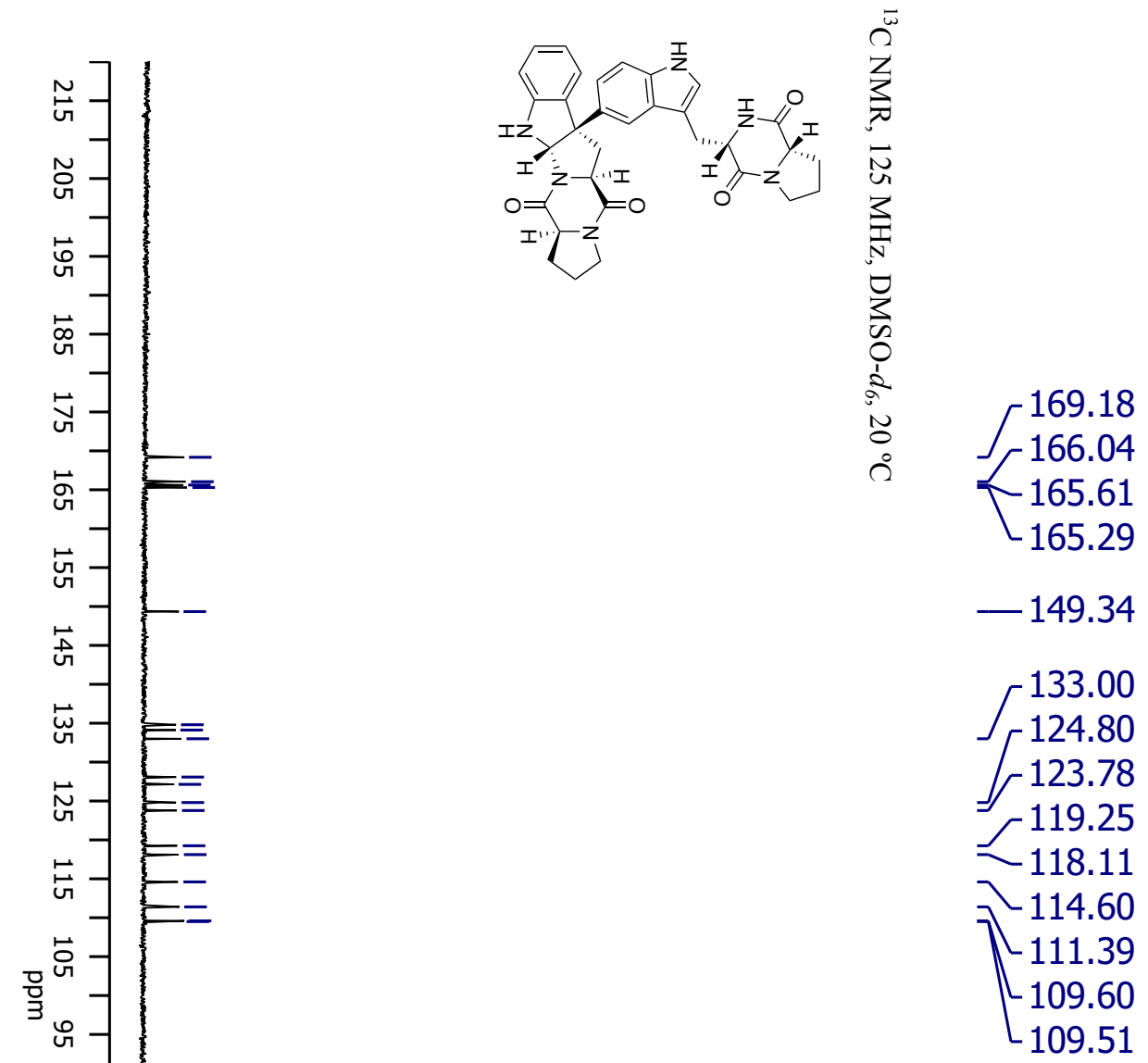

81.13

60.14

59.66

58.75

58.48

- 55.09

44.72

$-44.67$

覀

39.69 DMSO-D6

39.52 DMSO-D6

39.35 DMSO-D6

39.19 DMSO-D6

39.02 DMSO-D6

27.69

27.43

25.43

$-22.56$ 


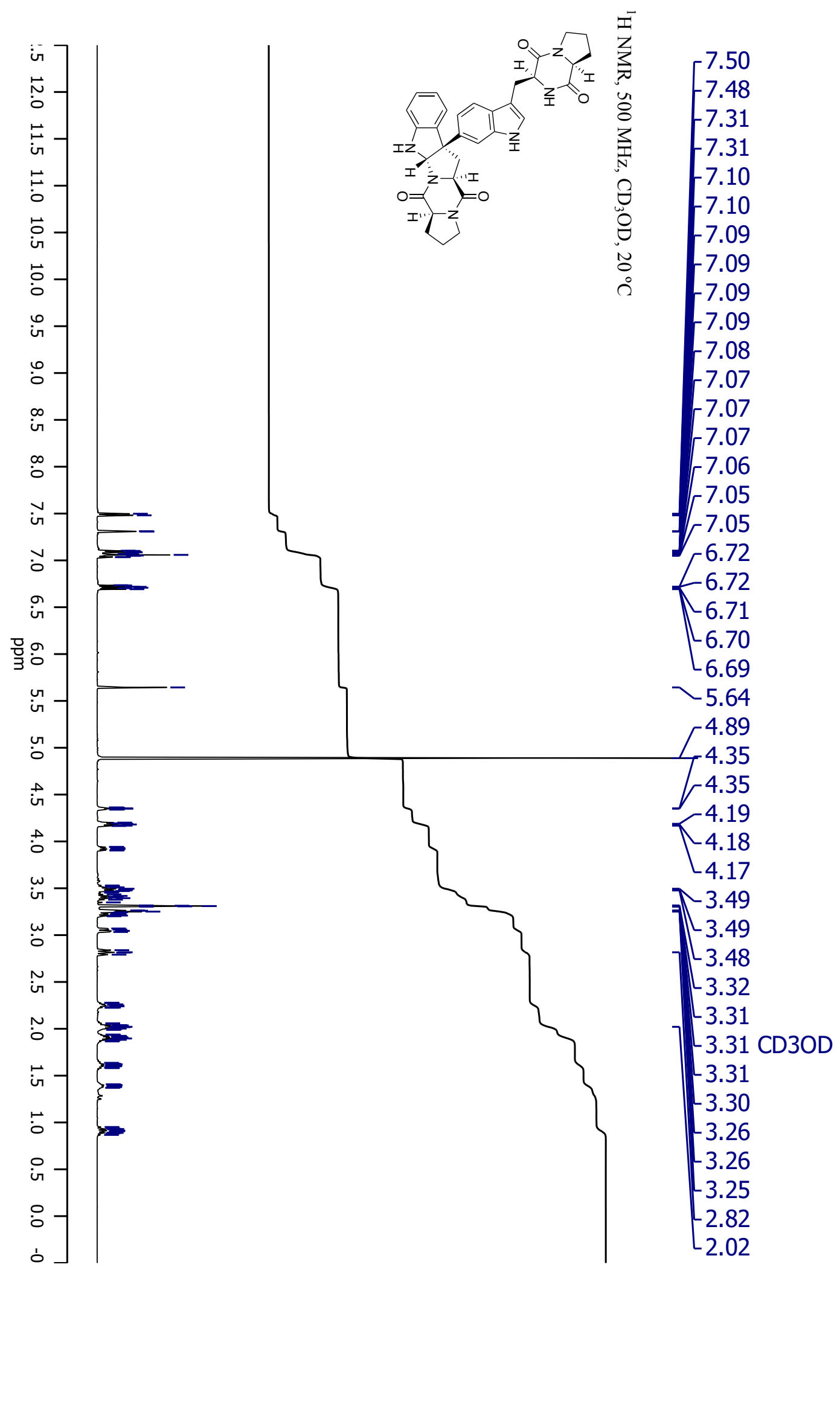




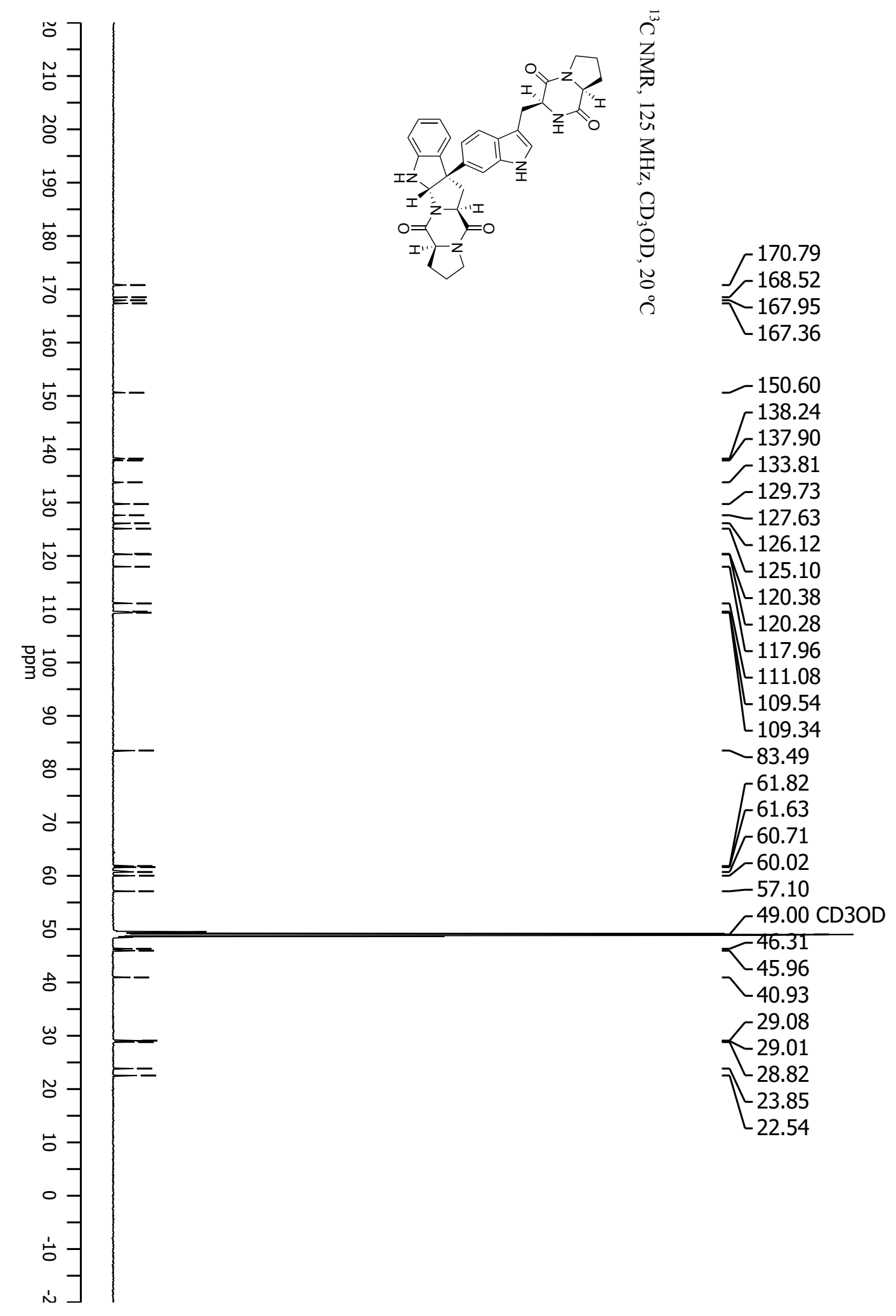




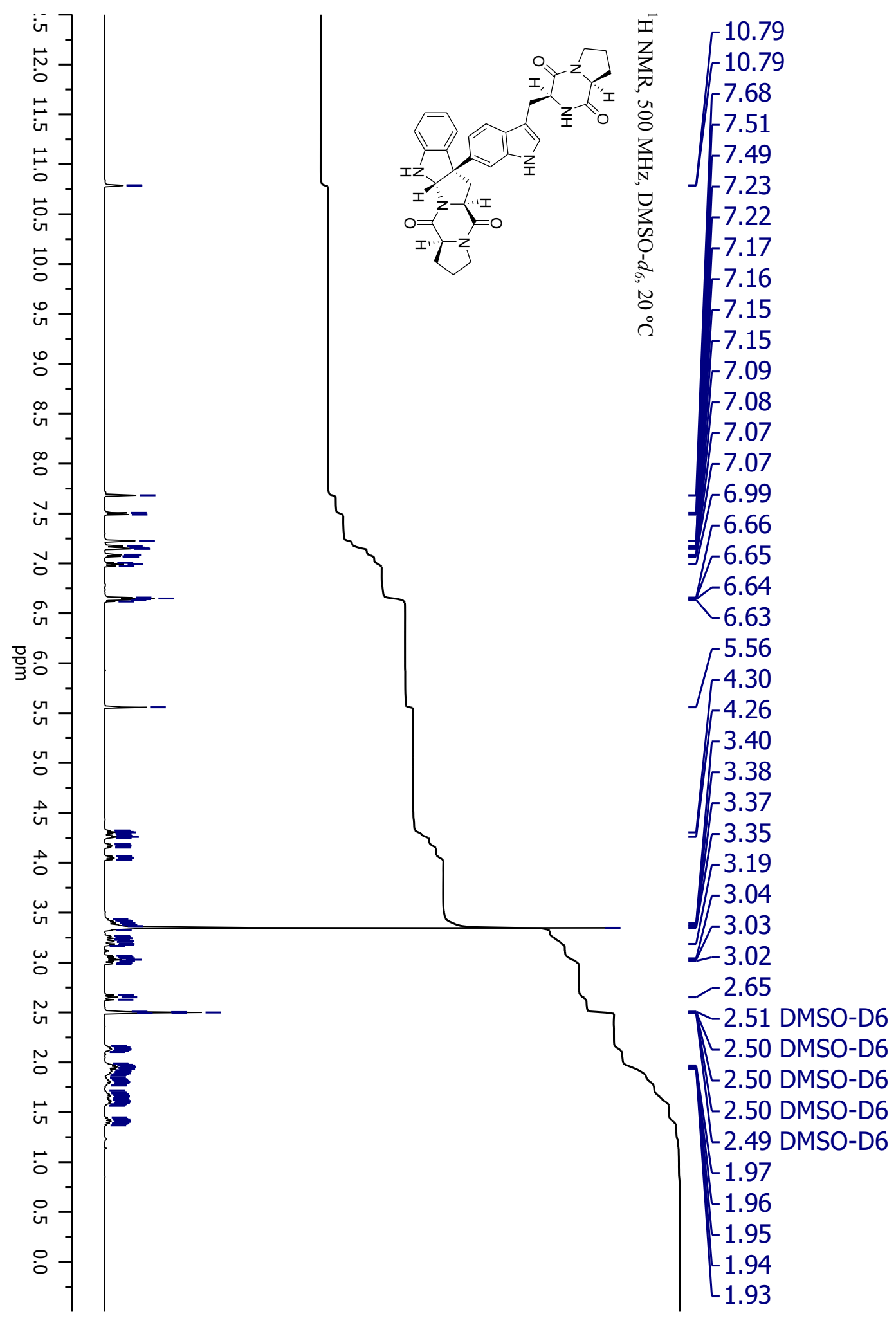




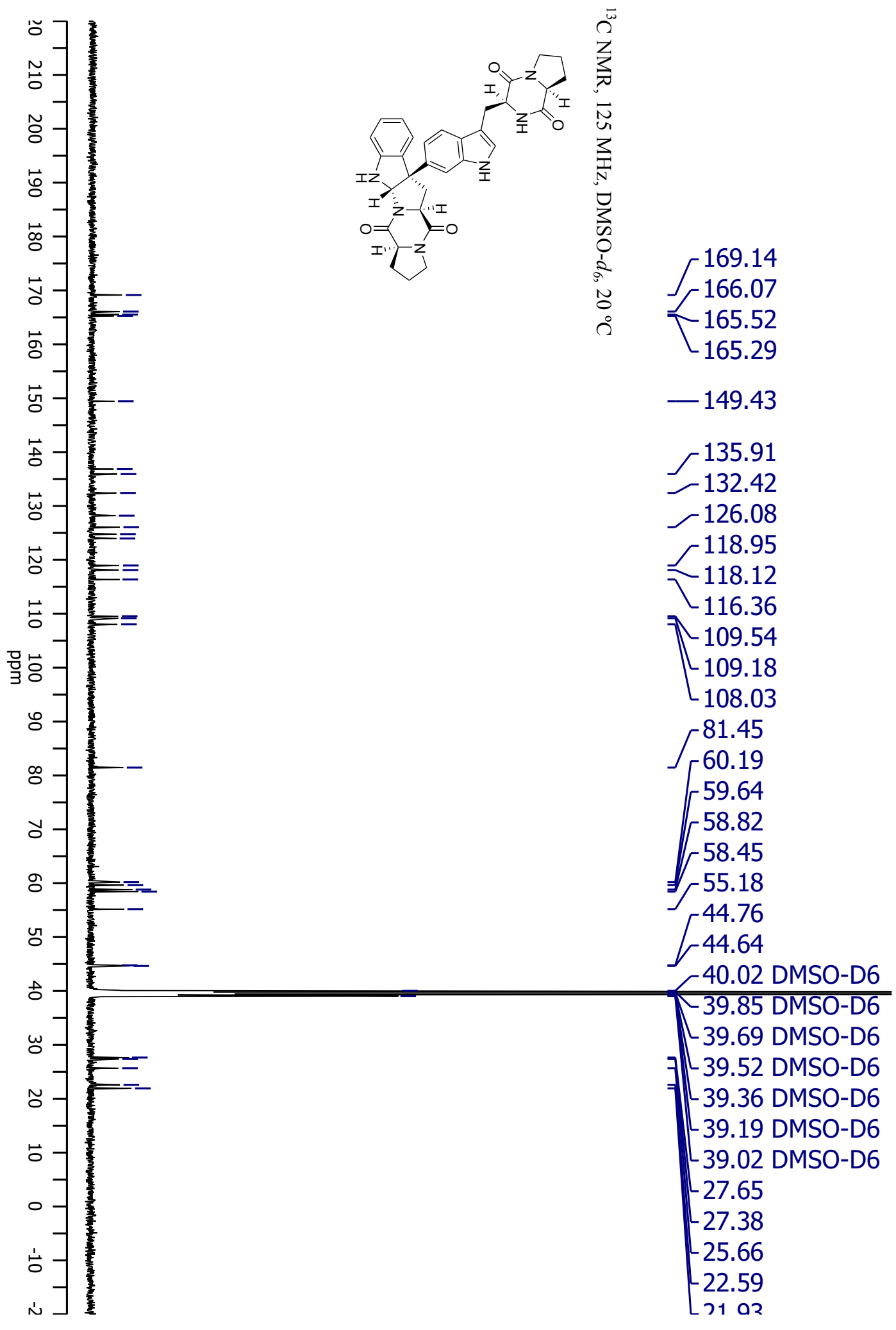


